\title{
Cyclopentanone Ring Expansion Leading to Functionalized $\delta$-Lactams: Short Synthesis of Simple Sedum Alkaloids ${ }^{\dagger}$
}

\author{
William A. Maio, Sandra Sinishtaj, and Gary H. Posner \\ Department of Chemistry, School of Arts and Sciences, The Johns Hopkins \\ University,Baltimore, MD 21218, USA
}

Supplementary Material:

I. Compound Characterization: pp. 2-16

II. NMR Spectra: pp. 17-96

III. X-Ray structures: pp. 97-114 


\section{Compound Characterization:}

\section{Supplemental Material}

Unless otherwise noted, all reactions were preformed in oven dried glassware and were stirred under an atmosphere of ultra high purity Argon. The tetrahydrofuran used was distilled from sodium/benzophenone, and the dichloromethane was distilled from calcium hydride. All other reagents were used as received from commercial suppliers, unless otherwise noted. Analytical TLC analysis was conducted on precoated glass-backed silica gel plates (Merck Kieselgel $60 \mathrm{~F}_{254}, 250 \mu$ thickness) and visualized with $p$-anisaldehyde or $\mathrm{KMnO}_{4}$ stains. Column chromatography was preformed using flash silica gel (particle size 230-400 mesh). Yields reported are for pure products (>95\% based on their chromatographic and spectroscopic homogeneity), and are unoptimized. NMR spectra were obtained on a Varian XL-400 spectrometer, operating at $400 \mathrm{MHz}$ for ${ }^{1} \mathrm{H}$ and 100 $\mathrm{MHz}$ for ${ }^{13} \mathrm{C}$, or a Bruker 400 spectrometer operating at the same frequencies. Chemical shifts are reported in $\mathrm{ppm}(\delta)$ and are referenced to $\mathrm{CDCl}_{3}(7.26$ and $77.0 \mathrm{ppm})$.

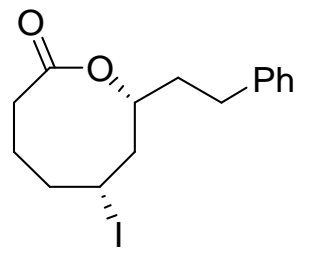

Lactone 4a (from epoxide 2a): ${ }^{1} \mathrm{H}$ NMR $\left(\mathrm{CDCl}_{3}\right) \delta 7.31-7.27(\mathrm{~m}, 2 \mathrm{H})$, 7.22-7.15 (m, 3H), 4.56-4.49 (m, 1H), 4.19-4.13 (m, 1H), 2.82-2.75

(m, 1H), 2.70-2.64 (m, 2H), 2.52-2.45 (m, 2H), 2.42-2.25 (m, 3H),

2.05-1.75 (m, 4H); ${ }^{13} \mathrm{C}\left(\mathrm{CDCl}_{3}\right) \delta 175.6,140.7,128.5,128.4,126.1,79.3,51.2,41.1,36.6$, 31.3, 28.2, 25.6; IR (neat) 2932, 1731, 1455, 1243, 1132, 750, $701 \mathrm{~cm}^{-1}$; HRMS (FAB, $\mathrm{M}+\mathrm{H}^{+}$) calcd. 359.0508 for $\mathrm{C}_{15} \mathrm{H}_{20} \mathrm{IO}_{2}$, found 359.0510. 


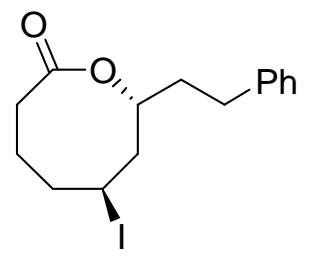

Lactone 4b (from epoxide 2a): ${ }^{1} \mathrm{H}$ NMR $\left(\mathrm{CDCl}_{3}\right) \delta$ 7.35-7.16 (m, 5H), 4.44-4.33 (m, 2H), 2.86-2.79 (m, 1H), 2.73-2.65 (m, 1H), 2.47-2.29 $(\mathrm{m}, 4 \mathrm{H}), 2.18-1.83(\mathrm{~m}, 6 \mathrm{H}) ;{ }^{13} \mathrm{C}\left(\mathrm{CDCl}_{3}\right) \delta 175.0,140.5,128.6,128.4$, 126.3, 77.1, 49.1, 37.3, 36.3, 31.3, 31.2, 28.1, 27.0; IR (neat) 2932, 1731, 1455, 1243, 1132, 750, $701 \mathrm{~cm}^{-1}$; HRMS (FAB, $\mathrm{M}+\mathrm{H}^{+}$) calcd. 359.0508 for $\mathrm{C}_{15} \mathrm{H}_{20} \mathrm{IO}_{2}$, found 359.0502 .

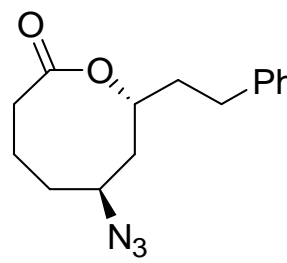

Lactone 5a (from epoxide 2a): ${ }^{1} \mathrm{H}$ NMR $\left(\mathrm{CDCl}_{3}\right) \delta$ 7.35-7.15 (m, 5H), 4.45-4.40 (m, 1H), 3.53-3.51 (m, 1H), 2.86-2.80 (m, 1H), 2.73-2.65 (m, 1H), 2.47-2.41 (m, 1H), 2.35-2.29 (m, 1H), 2.14-1.74 (m, 8H); ${ }^{13} \mathrm{C}$ $\left(\mathrm{CDCl}_{3}\right) \delta 174.9,140.5,128.6,128.4,126.3,74.3,58.7,41.5,36.6,32.3,32.0,31.3,24.5$; IR (neat) 2864, 2103, 1727, 1456, $1252 \mathrm{~cm}^{-1}$; HRMS (FAB, M+H') calcd. 274.1556 for $\mathrm{C}_{15} \mathrm{H}_{20} \mathrm{~N}_{3} \mathrm{O}_{2}$, found 274.1530 .

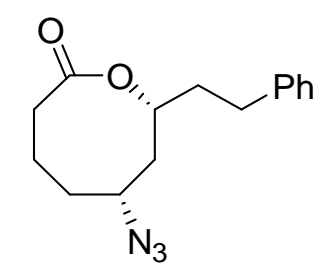

Lactone $\mathbf{5 b}$ (from epoxide 2a): ${ }^{1} \mathrm{H}$ NMR $\left(\mathrm{CDCl}_{3}\right) \delta$ 7.32-7.17 (m, 5H), 4.70-4.64 (m, 1H), 3.46-3.43 (m, 1H), 2.84-2.65 (m, 2H), 2.43-2.33 (m, 2H), 2.15-1.79 (m, 8H); ${ }^{13} \mathrm{C}\left(\mathrm{CDCl}_{3}\right) \delta 175.8,140.9,128.6,128.5$, 126.2, 76.2, 60.8, 42.9, 37.0, 33.5, 32.5, 31.5, 25.9; IR (neat) 2949, 2103, 1733, 1445, 1290, $1138 \mathrm{~cm}^{-1}$; HRMS (FAB, M+H') calcd. 274.1556 for $\mathrm{C}_{15} \mathrm{H}_{20} \mathrm{~N}_{3} \mathrm{O}_{2}$, found 274.1548.

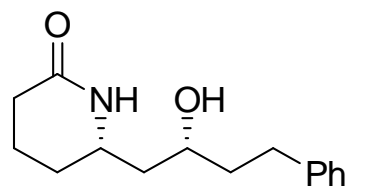

Lactam 6a (from epoxide 2a): ${ }^{1} \mathrm{H}$ NMR $\left(\mathrm{CDCl}_{3}\right) \delta 7.72(\mathrm{~s}, 1 \mathrm{H})$, 7.31-7.14 (m, 5H), $4.38(\mathrm{~s}, 1 \mathrm{H}), 3.87-3.81(\mathrm{~m}, 1 \mathrm{H}), 3.55-3.47(\mathrm{~m}$, $1 \mathrm{H}), 2.80-2.58(\mathrm{~m}, 2 \mathrm{H}), 2.39-2.20(\mathrm{~m}, 2 \mathrm{H}), 1.89-1.51(\mathrm{~m}, 6 \mathrm{H}), 1.43-1.19(\mathrm{~m}, 2 \mathrm{H}) ;{ }^{13} \mathrm{C}$ 
$\left(\mathrm{CDCl}_{3}\right) \delta 172.1,142.0,128.4,128.3,125.7,71.8,54.0,42.9,40.5,31.6,29.6,20.0 ; \mathrm{IR}$ (neat) 3313, 2941, 2858, 1634, 1460, $1344 \mathrm{~cm}^{-1}$; HRMS (FAB, M+H' ${ }^{+}$calcd. 248.1651 for $\mathrm{C}_{15} \mathrm{H}_{21} \mathrm{NO}_{2}$, found 248.1643 .

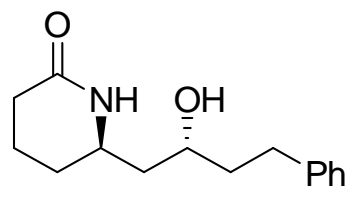

Lactam 6b (from epoxide 2a): ${ }^{1} \mathrm{H}$ NMR $\left(\mathrm{CDCl}_{3}\right) \delta 7.40(\mathrm{~s}, 1 \mathrm{H})$, 7.32-7.17 (m, 5H), 3.92-3.90 (m, 1H), 3.76-3.67 (m, 1H), $3.22(\mathrm{~s}$, $1 \mathrm{H}), 2.94-2.74(\mathrm{~m}, 1 \mathrm{H}), 2.70-2.57(\mathrm{~m}, 1 \mathrm{H}), 2.38-2.27(\mathrm{~m}, 2 \mathrm{H}), 1.91-1.56(\mathrm{~m}, 8 \mathrm{H}) ;{ }^{13} \mathrm{C}$ $\left(\mathrm{CDCl}_{3}\right) \delta 172.7,141.8,128.45,128.38,125.9,77.2,67.7,49.4,42.9,39.0,32.3,29.0$, 19.5; IR (neat) 3433, 2942, 2859, $1654 \mathrm{~cm}^{-1}$; HRMS (FAB, $\mathrm{M}+\mathrm{H}^{+}$) calcd. 248.1651 for $\mathrm{C}_{15} \mathrm{H}_{21} \mathrm{NO}_{2}$, found 248.1647 .

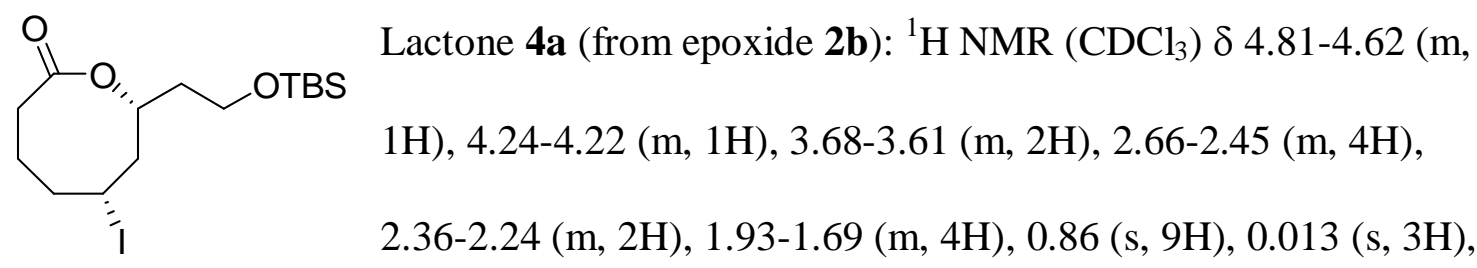
$0.005(\mathrm{~s}, 1 \mathrm{H}) ;{ }^{13} \mathrm{C}\left(\mathrm{CDCl}_{3}\right) \delta 175.0,77.3,58.3,50.9,40.6,38.0,30.5,27.8,25.8,25.7$, 18.2, -5.50, -5.52; IR (neat) 2958, 2863, 1723, 1476, 1362, 1238, 1096, 839, $773 \mathrm{~cm}^{-1}$; HRMS (FAB, $\mathrm{M}+\mathrm{H}^{+}$) calcd. 413.1009 for $\mathrm{C}_{15} \mathrm{H}_{29} \mathrm{IO}_{3} \mathrm{Si}$, found 413.0999 .

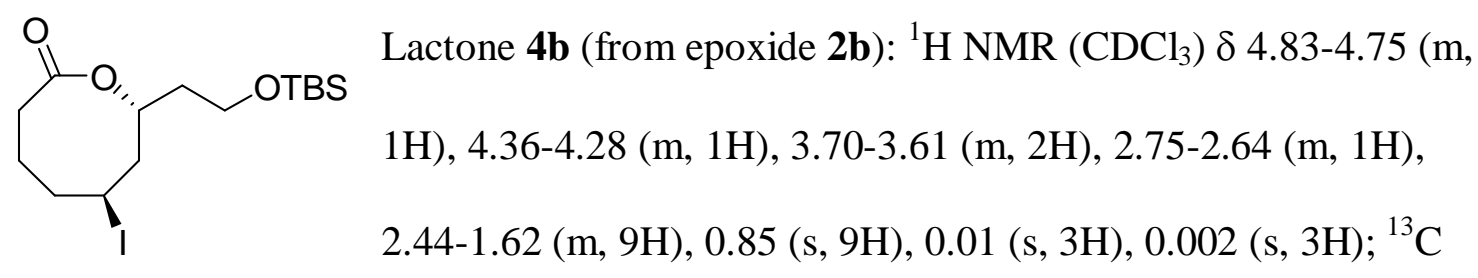


$\left(\mathrm{CDCl}_{3}\right) \delta 174.9,74.7,58.1,49.2,37.6,37.4,31.0,28.2,26.8,25.8,18.1,-5.5 ;$ IR (neat) 2945, 2860, 1726, 1458, 1360, 1251, 1190, 1092, 995, 824, $775 \mathrm{~cm}^{-1}$; HRMS (FAB, $\mathrm{M}+\mathrm{H}^{+}$) calcd. 413.1009 for $\mathrm{C}_{15} \mathrm{H}_{29} \mathrm{IO}_{3} \mathrm{Si}$, found 413.1025.

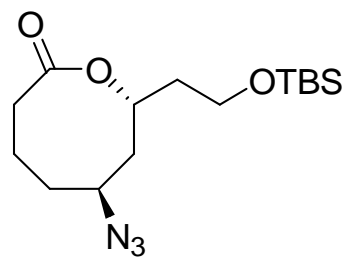

Lactone 5a (from epoxide $2 \mathbf{b}):{ }^{1} \mathrm{H} \mathrm{NMR}\left(\mathrm{CDCl}_{3}\right) \delta$ 4.89-4.84 (m, $1 \mathrm{H}), 3.74-3.66(\mathrm{~m}, 2 \mathrm{H}), 3.56-3.50(\mathrm{~m}, 1 \mathrm{H}), 2.71-2.64(\mathrm{~m}, 1 \mathrm{H})$, 2.53-2.48 (m, 1H), 2.17-1.72 (m, 8H), $0.88(\mathrm{~s}, 9 \mathrm{H}), 0.04(\mathrm{~s}, 3 \mathrm{H})$, $0.03(\mathrm{~s}, 3 \mathrm{H}) ;{ }^{13} \mathrm{C}\left(\mathrm{CDCl}_{3}\right) \delta 174.9,72.1,58.9,58.2,41.4,37.9,32.5,31.9,25.9,24.9,18.2$, -5.4, -5.9; IR (neat) 2948, 2929, 2854, 2096, 1726, 1461, 1253, 1092, 826, $769 \mathrm{~cm}^{-1}$; HRMS (FAB, $\mathrm{M}+\mathrm{H}^{+}$) calcd. 328.2057 for $\mathrm{C}_{15} \mathrm{H}_{29} \mathrm{~N}_{3} \mathrm{O}_{3} \mathrm{Si}$, found 328.2059.

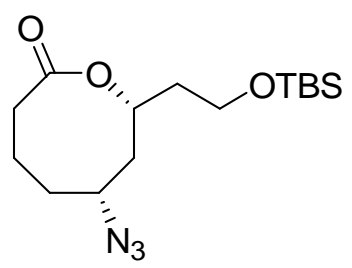

Lactone $\mathbf{5 b}$ (from epoxide $2 \mathbf{b}$ ): ${ }^{1} \mathrm{H}$ NMR $\left(\mathrm{CDCl}_{3}\right) \delta$ 4.86-4.78 (m, $1 \mathrm{H}), 3.71-3.60(\mathrm{~m}, 2 \mathrm{H}), 3.52-3.46(\mathrm{~m}, 1 \mathrm{H}), 2.67-2.60(\mathrm{~m}, 1 \mathrm{H})$, 2.49-2.44 (m, 1H), 2.13-2.02 (m, 2H), 1.97-1.90 (m, 1H), 1.87-1.68 (m, 5H), $0.85(\mathrm{~s}, 9 \mathrm{H}), 0.01(\mathrm{~s}, 3 \mathrm{H}),-0.01(\mathrm{~s}, 3 \mathrm{H}) ;{ }^{13} \mathrm{C}\left(\mathrm{CDCl}_{3}\right) \delta$ 174.8, 71.9, 58.8, 58.1, 41.3, 37.8, 32.4, 31.8, 25.8, 24.7, 18.0, -5.57, -5.60; IR (neat) 2967, 2939, 2854, 2086, 1736, 1461, 1253, 1186, 1092, 997, 931, 836, $779 \mathrm{~cm}^{-1}$, HRMS (FAB, M+H') calcd. 328.2057 for $\mathrm{C}_{15} \mathrm{H}_{29} \mathrm{~N}_{3} \mathrm{O}_{3} \mathrm{Si}$, found 328.2060.

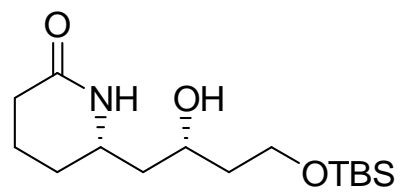

Lactam 6a (from epoxide 2b): ${ }^{1} \mathrm{H}$ NMR $\left(\mathrm{CDCl}_{3}\right) \delta 7.17$ (s, 1H), 4.02-3.96 (m, 1H), 3.87-3.76 (m, 2H), 3.57-3.51 (m, 1H), 2.37-

$2.30(\mathrm{~m}, 1 \mathrm{H}), 2.26-2.17(\mathrm{~m}, 1 \mathrm{H}), 1.90-1.80(\mathrm{~m}, 2 \mathrm{H}), 1.75-1.46(\mathrm{~m}, 5 \mathrm{H}), 0.86(\mathrm{~m}, 9 \mathrm{H})$, $0.040(\mathrm{~s}, 3 \mathrm{H}), 0.039(\mathrm{~s}, 3 \mathrm{H}) ;{ }^{13} \mathrm{C}\left(\mathrm{CDCl}_{3}\right) \delta 171.5,72.6,62.5,53.4,43.6,39.0,30.9,29.6$, 
25.7, 20.1, 18.0, 14.1, -5.64, -5.68; IR (neat) 3322, 3212, 2953, 2864, 1652, 1467, 1245, 1089, $830 \mathrm{~cm}^{-1}$; HRMS (FAB, M+H $\mathrm{H}^{+}$) calcd. 302.2152 for $\mathrm{C}_{15} \mathrm{H}_{32} \mathrm{NO}_{3} \mathrm{Si}$, found 302.2148 .

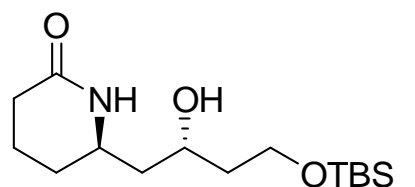

Lactam $6 \mathbf{b}$ (from epoxide $2 \mathbf{b}):{ }^{1} \mathrm{H}$ NMR $\left(\mathrm{CDCl}_{3}\right) \delta 6.71(\mathrm{~s}, 1 \mathrm{H})$, 4.08-4.02 (m, 1H), 3.93-3.78 (m, 3H), 3.73-3.67 (m, 1H), 2.41$2.23(\mathrm{~m}, 2 \mathrm{H}), 1.91-1.50(\mathrm{~m}, 7 \mathrm{H}), 1.44-1.37(\mathrm{~m}, 1 \mathrm{H}), 0.89(\mathrm{~s}, 9 \mathrm{H}), 0.08$ (s, 3H), 0.07 (s, $3 \mathrm{H}) ;{ }^{13} \mathrm{C}\left(\mathrm{CDCl}_{3}\right) \delta 172.2,69.4,63.1,49.3,43.3,38.0,31.2,29.2,25.8,19.6,18.1,-5.59$, -5.63; IR (neat) 3330, 2930, 2901, 2849, 1645, 1246, 1090, $832 \mathrm{~cm}^{-1}$; HRMS (FAB, $\mathrm{M}+\mathrm{H}^{+}$) calcd. 302.2152 for $\mathrm{C}_{15} \mathrm{H}_{32} \mathrm{NO}_{3} \mathrm{Si}$, found 302.2148.

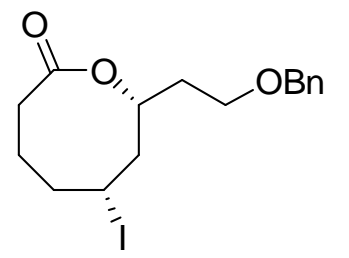

Lactone 4a (from epoxide 2c): ${ }^{1} \mathrm{H}$ NMR $\left(\mathrm{CDCl}_{3}\right) \delta$ 7.37-7.27 (m, $5 \mathrm{H}), 4.83-4.76(\mathrm{~m}, 1 \mathrm{H}), 4.47(\mathrm{~s}, 2 \mathrm{H}), 4.25-4.19(\mathrm{~m}, 1 \mathrm{H}), 3.60-3.49$ (m, 2H), 2.68-2.42 (m, 4H), 2.36-2.22 (m, 2H), 1.94-1.78 (m, 4H);

${ }^{13} \mathrm{C}\left(\mathrm{CDCl}_{3}\right) \delta 175.2,138.1,128.4,127.7,73.2,65.5,50.9,40.7,35.4,30.9,27.9,25.6 ; \mathrm{IR}$ (neat) 2921, 2866, 1740, 1449, 1356, 1245, 1145, $1107 \mathrm{~cm}^{-1}$; HRMS (FAB, M+H') calcd. 389.0614 for $\mathrm{C}_{16} \mathrm{H}_{22} \mathrm{IO}_{3}$, found 389.0613 .

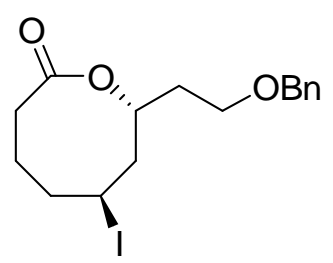

Lactone 4b (from epoxide $2 \mathbf{c}$ ): ${ }^{1} \mathrm{H}$ NMR $\left(\mathrm{CDCl}_{3}\right) \delta$ 7.38-7.27 (m, $5 \mathrm{H}), 4.80-4.73(\mathrm{~m}, 1 \mathrm{H}), 4.52-4.44(\mathrm{~m}, 2 \mathrm{H}), 4.32-4.26(\mathrm{~m}, 1 \mathrm{H}), 3.63-$ $3.51(\mathrm{~m}, 2 \mathrm{H}), 2.69-2.61(\mathrm{~m}, 1 \mathrm{H}), 2.48-2.12(\mathrm{~m}, 5 \mathrm{H}), 1.95-1.84(\mathrm{~m}$, $4 \mathrm{H}) ;{ }^{13} \mathrm{C}\left(\mathrm{CDCl}_{3}\right) \delta 174.9,138.0,128.4,127.8,74.9,73.2,65.3,49.3,37.8,35.0,31.1$, 28.1, 26.1; IR (neat) 2935, 2866, 1736, 1458, 1352, 1240, 1185, 1111, $1018 \mathrm{~cm}^{-1}$; HRMS (FAB, $\mathrm{M}+\mathrm{H}^{+}$) calcd. 389.0614 for $\mathrm{C}_{16} \mathrm{H}_{22} \mathrm{IO}_{3}$, found 389.0609 . 


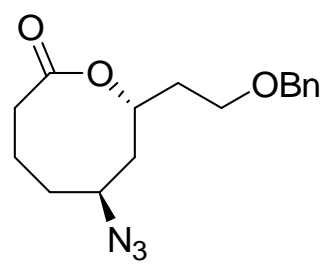

Lactone 5a (from epoxide 2c): ${ }^{1} \mathrm{H}$ NMR $\left(\mathrm{CDCl}_{3}\right) \delta$ 7.37-7.28 (m, $5 \mathrm{H}), 4.85-4.79(\mathrm{~m}, 1 \mathrm{H}), 4.52-4.42(\mathrm{~m}, 2 \mathrm{H}), 3.62-3.48(\mathrm{~m}, 3 \mathrm{H}), 2.65-$ $2.57(\mathrm{~m}, 1 \mathrm{H}), 2.48-2.43(\mathrm{~m}, 1 \mathrm{H}), 2.15-1.71(\mathrm{~m}, 8 \mathrm{H}) ;{ }^{13} \mathrm{C}\left(\mathrm{CDCl}_{3}\right) \delta$ 175.0, 138.0, 128.5, 127.8, 73.3, 72.3, 65.3, 58.8, 41.4, 35.4, 32.4, 31.9, 24.8; IR (neat) 2926, 2868, 2097, 1728, 1452, 1360, 1249, $1098 \mathrm{~cm}^{-1}$; HRMS (FAB, M+H') calcd. 304.1661 for $\mathrm{C}_{16} \mathrm{H}_{22} \mathrm{~N}_{3} \mathrm{O}_{3}$, found 304.1656.

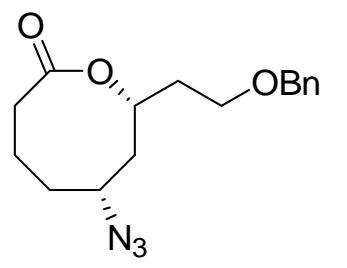

Lactone $5 \mathbf{b}$ (from epoxide $2 \mathbf{c}):{ }^{1} \mathrm{H}$ NMR $\left(\mathrm{CDCl}_{3}\right) \delta$ 7.40-7.29 (m, $5 \mathrm{H}), 4.98-4.91(\mathrm{~m}, 1 \mathrm{H}), 4.51(\mathrm{~s}, 2 \mathrm{H}), 3.66-3.50(\mathrm{~m}, 3 \mathrm{H}), 2.57-2.51$ (m, 1H), 2.49-2.38 (m, 1H), 2.17-1.76 (m, 8H); ${ }^{13} \mathrm{C}\left(\mathrm{CDCl}_{3}\right) \delta 175.3$, $138.1,128.4,127.8,127.7,74.2,73.3,65.7,60.6,42.5,35.8,33.0,32.0,25.4$; IR (neat) 2944, 2868, 2092, 1733, 1459, 1365, 1243, 1142, 1093, $743 \mathrm{~cm}^{-1}$; HRMS (FAB, M+H') calcd. 304.1661 for $\mathrm{C}_{16} \mathrm{H}_{22} \mathrm{~N}_{3} \mathrm{O}_{3}$, found 304.1668.

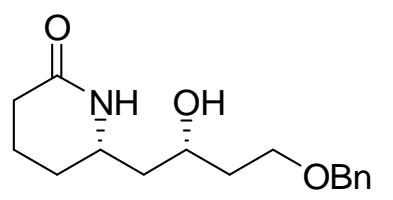
Lactam 6a (from epoxide 2c): ${ }^{1} \mathrm{H}$ NMR $\left(\mathrm{CDCl}_{3}\right) \delta$ 7.38-7.29 (m, $5 \mathrm{H}), 4.52(\mathrm{~s}, 2 \mathrm{H}), 4.05-3.99(\mathrm{~m}, 1 \mathrm{H}), 3.76-3.55(\mathrm{~m}, 3 \mathrm{H}), 2.90(\mathrm{~s}$, $1 \mathrm{H}), 2.44-2.32(\mathrm{~m}, 1 \mathrm{H}), 2.30-2.23(\mathrm{~m}, 1 \mathrm{H}), 1.90-1.21(\mathrm{~m}, 8 \mathrm{H}) ;{ }^{13} \mathrm{C}\left(\mathrm{CDCl}_{3}\right) \delta 171.7$, $137.6,128.5,127.9,127.7,73.4,73.0,68.9,53.4,43.4,37.1,30.9,29.6,20.1$; IR (neat) 3324, 2968, 2896, 1641, 1476, 1451, 1409, 1351, 1310, $1094 \mathrm{~cm}^{-1}$; HRMS (FAB, M+H') calcd. 278.1756 for $\mathrm{C}_{16} \mathrm{H}_{24} \mathrm{NO}_{3}$, found 278.1746. 


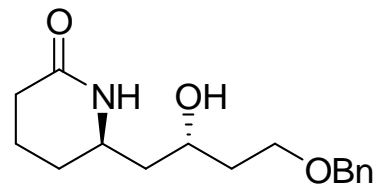

Lactam 6b (from epoxide $2 \mathbf{c}):{ }^{1} \mathrm{H}$ NMR $\left(\mathrm{CDCl}_{3}\right) \delta$ 7.38-7.30 (m, $5 \mathrm{H}), 6.77(\mathrm{~s}, 1 \mathrm{H}), 4.52(\mathrm{~s}, 1 \mathrm{H}), 4.07-4.01(\mathrm{~m}, 1 \mathrm{H}), 3.75-3.63(\mathrm{~m}$, $3 \mathrm{H}), 3.35(\mathrm{~s}, 1 \mathrm{H}), 2.39-2.23(\mathrm{~m}, 2 \mathrm{H}), 1.92-1.82(\mathrm{~m}, 3 \mathrm{H}), 1.76-1.54(\mathrm{~m}, 4 \mathrm{H}), 1.44-1.36(\mathrm{~m}$, $1 \mathrm{H}) ;{ }^{13} \mathrm{C}\left(\mathrm{CDCl}_{3}\right) \delta 172.2,137.6,128.5,127.9,127.7,77.2,73.5,69.3,68.6,49.4,43.1$, 36.2, 29.2, 19.6; IR (neat) 3337, 2948, 2865, 1648, $1095 \mathrm{~cm}^{-1}$; HRMS (FAB, M+H') calcd. 278.1756 for $\mathrm{C}_{16} \mathrm{H}_{24} \mathrm{NO}_{3}$, found 278.1777.

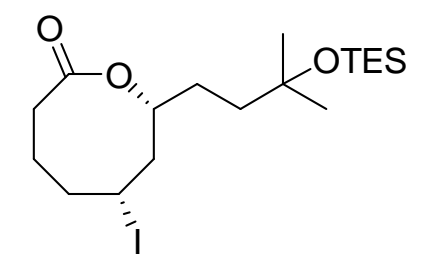

Lactone 4a (from epoxide 2d): ${ }^{1} \mathrm{H} \mathrm{NMR}\left(\mathrm{CDCl}_{3}\right) \delta$ 4.62-4.59 (m, $1 \mathrm{H}), 4.24-4.13(\mathrm{~m}, 1 \mathrm{H}), 2.75-2.62(\mathrm{~m}, 1 \mathrm{H}), 2.56-2.25(\mathrm{~m}, 5 \mathrm{H})$, 2.01-1.85 (m, 2H), 1.71-1.32 (m, 4H), $1.18(\mathrm{~s}, 3 \mathrm{H}), 1.16(\mathrm{~s}, 3 \mathrm{H})$, $0.91(\mathrm{t}, J=10.4 \mathrm{~Hz}, 9 \mathrm{H}), 0.53(\mathrm{q}, J=10.8 \mathrm{~Hz}, 6 \mathrm{H}) ;{ }^{13} \mathrm{C}\left(\mathrm{CDCl}_{3}\right) \delta 175.9,81.0,72.7,51.6$, 41.6, 40.5, 31.9, 30.1, 29.9, 29.5, 28.6, 26.0, 7.1, 6.7; IR (neat) 2953, 2871, 1739, 1450, 1360, 1236, 1153, 1046, $715 \mathrm{~cm}^{-1}$; HRMS (FAB, M+H') calcd. 455.1479 for $\mathrm{C}_{18} \mathrm{H}_{36} \mathrm{IO}_{3} \mathrm{Si}$, found 455.1461 .

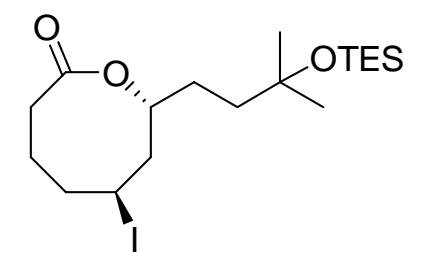

Lactone 4b (from epoxide 2d): ${ }^{1} \mathrm{H} \mathrm{NMR}\left(\mathrm{CDCl}_{3}\right) \delta$ 4.94-4.37 (m, $2 \mathrm{H}), 2.70-2.62(\mathrm{~m}, 1 \mathrm{H}), 2.48-2.35(\mathrm{~m}, 3 \mathrm{H}), 2.18-2.13(\mathrm{~m}, 2 \mathrm{H})$, $1.98-1.39(\mathrm{~m}, 6 \mathrm{H}), 1.21(\mathrm{~s}, 3 \mathrm{H}), 1.19(\mathrm{~s}, 3 \mathrm{H}), 0.93(\mathrm{t}, J=10.4 \mathrm{~Hz}$, 9H), $0.56(\mathrm{q}, J=9.6 \mathrm{~Hz}, 6 \mathrm{H}) ;{ }^{13} \mathrm{C}\left(\mathrm{CDCl}_{3}\right) \delta 175.1,79.3,72.7,49.1,40.7,37.2,31.3,30.3$, 29.9, 29.4, 28.1, 27.6, 7.1, 6.7; IR (neat) 2989, 2868, 1733, 1559, 1443, $621 \mathrm{~cm}^{-1}$; HRMS (FAB, $\mathrm{M}+\mathrm{H}^{+}$) calcd. 455.1479 for $\mathrm{C}_{18} \mathrm{H}_{36} \mathrm{IO}_{3} \mathrm{Si}$, found 455.1464 . 


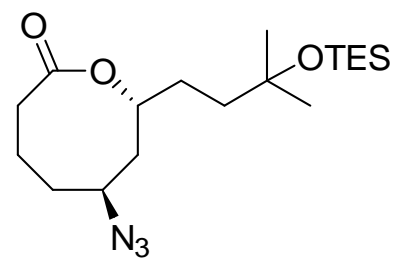

Lactone 5a (from epoxide 2d): ${ }^{1} \mathrm{H}$ NMR $\left(\mathrm{CDCl}_{3}\right) \delta$ 4.55-4.49 (m, $1 \mathrm{H}), 3.60-3.56(\mathrm{~m}, 1 \mathrm{H}), 2.68-2.59(\mathrm{~m}, 1 \mathrm{H}), 2.54-2.48(\mathrm{~m}, 1 \mathrm{H})$, 2.09-2.05 (m, 2H), 1.96-1.78 (m, 5H), 1.72-1.64 (m, 1H), 1.61-

$1.57(\mathrm{~m}, 1 \mathrm{H}), 1.45-1.34(\mathrm{~m}, 1 \mathrm{H}), 1.21(\mathrm{~s}, 3 \mathrm{H}), 1.20(\mathrm{~s}, 3 \mathrm{H}), 0.93(\mathrm{t}, J=8.0 \mathrm{~Hz}, 9 \mathrm{H}), 0.56$ $(\mathrm{q}, J=7.6 \mathrm{~Hz}, 6 \mathrm{H}) ;{ }^{13} \mathrm{C}\left(\mathrm{CDCl}_{3}\right) \delta 175.0,76.6,72.8,58.7,41.5,40.8,32.3,32.1,30.29$, 30.25, 29.4, 24.6, 7.1, 6.7; IR (neat) 2954, 2872, 2104, 1732, 1460, 1361, 1245, 1031, $725 \mathrm{~cm}^{-1}$; HRMS (FAB, $\mathrm{M}+\mathrm{H}^{+}$) calcd. 370.2526 for $\mathrm{C}_{18} \mathrm{H}_{36} \mathrm{~N}_{3} \mathrm{O}_{3} \mathrm{Si}$, found 370.2516 .

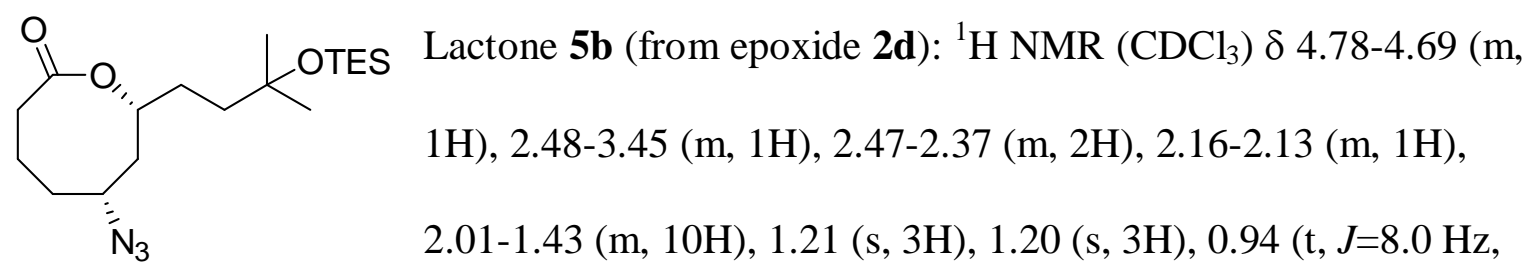
9H), $0.56(\mathrm{q}, J=8.0 \mathrm{~Hz}, 6 \mathrm{H}) ;{ }^{13} \mathrm{C}\left(\mathrm{CDCl}_{3}\right) \delta 176.0,77.9,72.8,61.0,43.2,40.6,33.9,32.9$, 30.3, 30.2, 29.5, 26.4, 7.1, 6.7; IR (neat) 2963, 2872, 2096, 1741, 1452, 1361, 1245, 1047, $737 \mathrm{~cm}^{-1}$; HRMS (FAB, $\mathrm{M}+\mathrm{H}^{+}$) calcd. 370.2526 for $\mathrm{C}_{18} \mathrm{H}_{36} \mathrm{~N}_{3} \mathrm{O}_{3} \mathrm{Si}$, found 370.2508 .

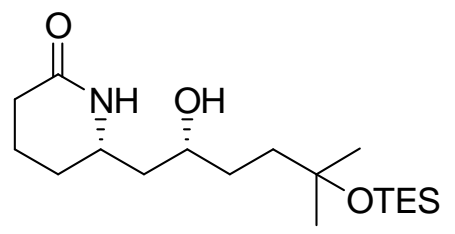

Lactam 6a (from epoxide 2d): ${ }^{1} \mathrm{H}$ NMR $\left(\mathrm{CDCl}_{3}\right) \delta 7.33$ (s, $1 \mathrm{H}), 3.79-3.76(\mathrm{~m}, 1 \mathrm{H}), 3.58-3.45(\mathrm{~m}, 1 \mathrm{H}), 2.40-2.17(\mathrm{~m}, 2 \mathrm{H})$, $1.86-1.25(\mathrm{~m}, 10 \mathrm{H}), 1.21(\mathrm{~s}, 6 \mathrm{H}), 0.93(\mathrm{t}, J=8.1 \mathrm{~Hz}, 9 \mathrm{H}), 0.57$ $(\mathrm{q}, J=10.8,6 \mathrm{H}) ;{ }^{13} \mathrm{C}\left(\mathrm{CDCl}_{3}\right) \delta 171.8,73.6,72.5,53.7,43.3,40.8,33.5,30.9,29.80$, 29.77, 29.6, 20.1, 7.0, 6.6; IR (neat) 3324, 3267, 2954, 2871, 1660, 1463, 1364, 1232, 1076, 1034, 1010, 960, 738, $713 \mathrm{~cm}^{-1}$; HRMS (FAB, M+H' $)$ calcd. 344.2621 for $\mathrm{C}_{18} \mathrm{H}_{38} \mathrm{NO}_{3}$, found 344.2603. 


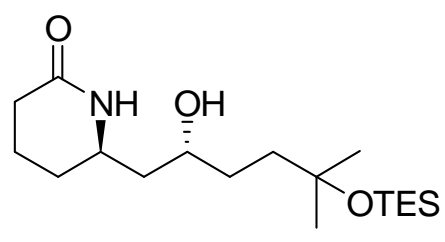

Lactam 6b (from epoxide 2d): ${ }^{1} \mathrm{H}$ NMR $\left(\mathrm{CDCl}_{3}\right) \delta 6.99(\mathrm{~s}$, $1 \mathrm{H}), 3.78(\mathrm{~s}, 1 \mathrm{H}), 3.69-3.67(\mathrm{~m}, 1 \mathrm{H}), 2.37-2.26(\mathrm{~m}, 2 \mathrm{H}), 1.92-$ $1.84(\mathrm{~m}, 2 \mathrm{H}) 1.70-1.37(\mathrm{~m}, 8 \mathrm{H}), 1.22(\mathrm{~s}, 6 \mathrm{H}), 0.93(\mathrm{t}, J=6.0$ $\mathrm{Hz}, 9 \mathrm{H}), 0.57$ (q, $J=7.6 \mathrm{~Hz}, 6 \mathrm{H}) ;{ }^{13} \mathrm{C}\left(\mathrm{CDCl}_{3}\right) \delta 172.5,73.5,68.6,49.3,43.1,41.4,32.2$, 31.2, 29.8, 29.2, 19.5, 7.0, 6.6; IR (neat) 3349, 2954, 2871, 1652, 1459, 1405, 1364, 1240, 1158, 1051, $713 \mathrm{~cm}^{-1}$; HRMS (FAB, $\mathrm{M}+\mathrm{H}^{+}$) calcd. 344.2621 for $\mathrm{C}_{18} \mathrm{H}_{38} \mathrm{NO}_{3}$, found 344.2627.

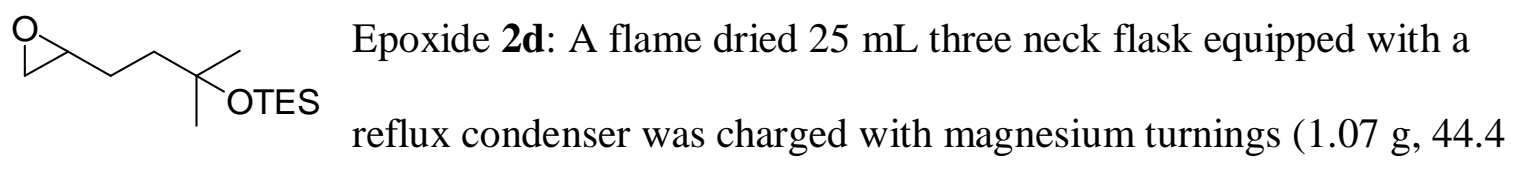
mmol) a small crystal of iodine and $7.0 \mathrm{~mL}$ of anhydrous ether. The flask was cooled to 0 ${ }^{\circ} \mathrm{C}$ and 4-bromobutene (1.0 g, $\left.7.40 \mathrm{mmol}\right)$ was added dropwise over the course of $5 \mathrm{~min}$. The solution immediately turned from red to colorless. This contents of the flask were allowed to warm to room temp and stir for $4 \mathrm{hr}$ before reagent grade acetone $(0.363 \mathrm{~mL}$, $14.8 \mathrm{mmol}$ ) was added neat. After an additional $2 \mathrm{hr}$ of stirring, the reaction was quenched by the slow addition of $1 \mathrm{~N} \mathrm{NaOH}$ solution. The reaction mixture was passed over celite to remove excess magnesium turnings, and then extracted with ether $(3 \mathrm{x} 50$ $\mathrm{mL}$ ). The combined organics were dried over $\mathrm{MgSO}_{4}$ and concentrated in vacuo, and taken on to the next step without further purification.

The tertiary alcohol was then dissolved in $20 \mathrm{~mL}$ of anhydrous $\mathrm{CH}_{2} \mathrm{Cl}_{2}$ and cooled to $-78{ }^{\circ} \mathrm{C} .2,6$-Lutadine $(2.15 \mathrm{~mL}, 18.5 \mathrm{mmol})$ was then added, followed by TESOTf (3.34 mL, $14.8 \mathrm{mmol}$ ) dropwise. After $2 \mathrm{hr}$, the reaction was quenched with water, and 
then extracted with $\mathrm{CH}_{2} \mathrm{Cl}_{2}(3 \times 50 \mathrm{~mL})$. The combined organics were dried over $\mathrm{MgSO}_{4}$ and concentrated in vacuo, and taken on to the next step without further purification. TES ether was dissolved in $20 \mathrm{~mL}$ of anhydrous $\mathrm{CH}_{2} \mathrm{Cl}_{2}$ and cooled to $0{ }^{\circ} \mathrm{C}$. To this was added $\mathrm{mCPBA}(4.15 \mathrm{~g}, 18.5 \mathrm{mmol})$ dissolved in $15 \mathrm{~mL}$ of ether. The reaction was allowed to warm to room temp and stir overnight. The mixture was diluted with $\mathrm{CH}_{2} \mathrm{Cl}_{2}(50 \mathrm{~mL})$ and washed first with a saturated sodium bisulfite solution (1x $\left.50 \mathrm{~mL}\right)$, and then a saturated sodium bicarbonate solution $(1 \times 50 \mathrm{~mL})$. The combined organics were dried over $\mathrm{MgSO}_{4}$ and concentrated in vacuo. The crude product was purified by silica gel chromatography ( $80 \%$ hexanes, $20 \%$ ethyl acetate) to give epoxide $2 \mathbf{d}(1.21 \mathrm{~g}$, $67 \%)$ as a colorless oil. ${ }^{1} \mathrm{H}$ NMR $\left(\mathrm{CDCl}_{3}\right) \delta 2.89-2.86(\mathrm{~m}, 1 \mathrm{H}), 2.72-2.69(\mathrm{~m}, 1 \mathrm{H}), 2.44-$ $2.41(\mathrm{~m}, 1 \mathrm{H}), 1.62-1.49(\mathrm{~m}, 4 \mathrm{H}), 1.19(\mathrm{~s}, 3 \mathrm{H}), 1.17(\mathrm{~s}, 3 \mathrm{H}), 0.91(\mathrm{t}, J=10.0 \mathrm{~Hz}, 9 \mathrm{H}), 0.54$ (q, $J=10.4 \mathrm{~Hz}, 6 \mathrm{H}) ;{ }^{13} \mathrm{C}\left(\mathrm{CDCl}_{3}\right) \delta 72.7,52.6,47.1,40.7,29.9,29.6,27.5,7.0,6.7 ; \mathrm{IR}$ (neat) 2955, 2919, 2861, 2884, 1467, 1249, 1102, 838, $773 \mathrm{~cm}^{-1}$; HRMS (EI, M⿻) calcd. 244.1858 for $\mathrm{C}_{13} \mathrm{H}_{28} \mathrm{O}_{2} \mathrm{Si}^{+}$, found 244.1832.

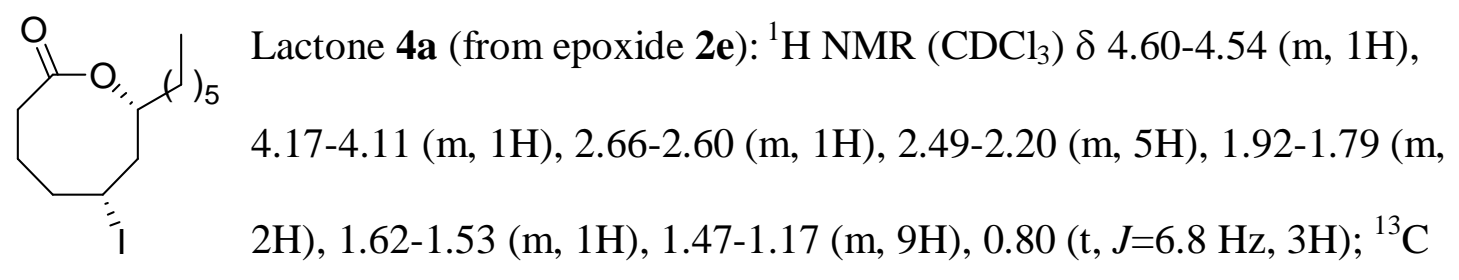
$\left(\mathrm{CDCl}_{3}\right) \delta 175.7,80.2,51.3,41.4,34.9,31.6,31.4,28.7,28.4,26.0,25.1,22.3,13.9 ; \mathrm{IR}$ (neat) $2966,2857,2848,1715,1468,1354,1248,1179,1081,973,726 \mathrm{~cm}^{-1}$; HRMS

(FAB, $\mathrm{M}+\mathrm{H}^{+}$) calcd. 339.0821 for $\mathrm{C}_{13} \mathrm{H}_{24} \mathrm{IO}_{2}$, found 339.0825.

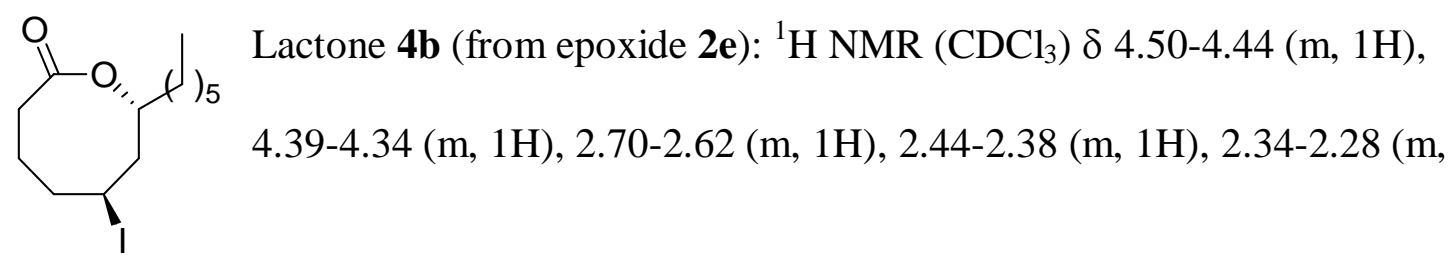


$2 \mathrm{H}), 2.17-2.06(\mathrm{~m}, 2 \mathrm{H}), 2.00-1.84(\mathrm{~m}, 2 \mathrm{H}), 1.71-1.64(\mathrm{~m}, 2 \mathrm{H}), 1.52-1.21(\mathrm{~m}, 8 \mathrm{H}), 0.84(\mathrm{t}$, $J=6.8 \mathrm{~Hz}, 3 \mathrm{H}) ;{ }^{13} \mathrm{C}\left(\mathrm{CDCl}_{3}\right) \delta 174.9,78.5,53.3,48.8,37.0,35.0,31.5,31.1,28.8,28.0$, 27.7, 25.3, 22.4, 13.9; IR (neat) 2956, 2929, 2857, 1716, 1558, 1458, 1240, 1092, 1010, $947 \mathrm{~cm}^{-1}$; HRMS $\left(\mathrm{FAB}, \mathrm{M}+\mathrm{H}^{+}\right)$calcd. 339.0821 for $\mathrm{C}_{13} \mathrm{H}_{24} \mathrm{IO}_{2}$, found 339.0821 .

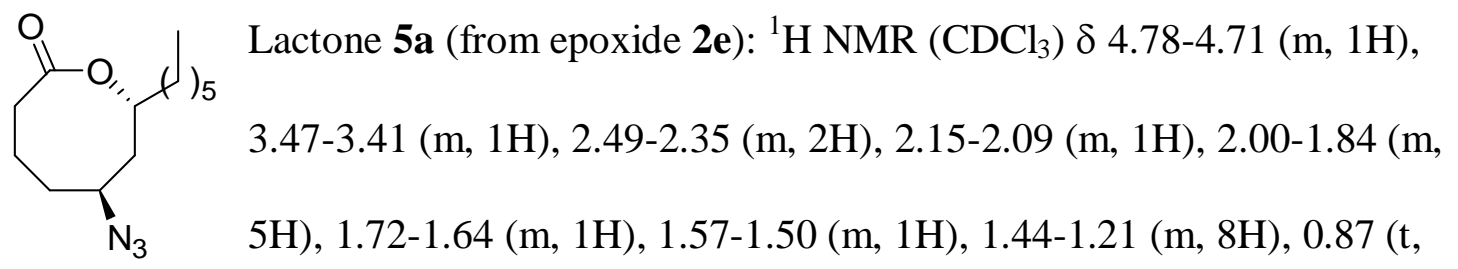
$J=7.2 \mathrm{~Hz}, 3 \mathrm{H}) ;{ }^{13} \mathrm{C}\left(\mathrm{CDCl}_{3}\right) \delta 176.0,77.3,61.0,43.0,35.4,33.8,32.8,31.6,28.9,26.3$, 25.3, 22.5, 14.0; IR (neat) 2936, 2861, 2089, 1735, 1457, 1355, 1318, 1248, 1153, 1052, $1008 \mathrm{~cm}^{-1}$; HRMS $\left(\mathrm{FAB}, \mathrm{M}+\mathrm{H}^{+}\right.$) calcd. 254.1869 for $\mathrm{C}_{13} \mathrm{H}_{24} \mathrm{~N}_{3} \mathrm{O}_{2}$, found 254.1874.

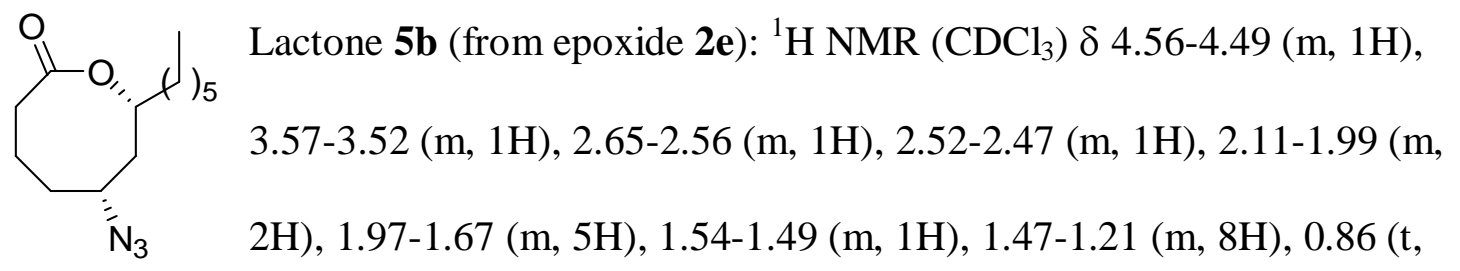
$J=6.8 \mathrm{~Hz}, 3 \mathrm{H}) ;{ }^{13} \mathrm{C}\left(\mathrm{CDCl}_{3}\right) \delta 175.0,75.8,58.7,41.4,35.3,32.2,32.0,31.6,28.9,25.4$, 24.5, 22.4, 13.9; IR (neat) 2930, 2861, 2095, 1729, 1457, 1330, 1248, 1204, 1128, 1008 $\mathrm{cm}^{-1}$; HRMS (FAB, $\mathrm{M}+\mathrm{H}^{+}$) calcd. 254.1869 for $\mathrm{C}_{13} \mathrm{H}_{24} \mathrm{~N}_{3} \mathrm{O}_{2}$, found 254.1862 .

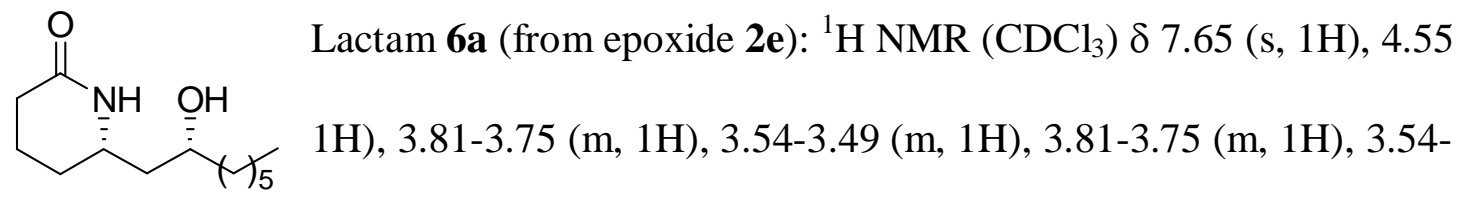
$3.45(\mathrm{~m}, 1 \mathrm{H}), 2.38-2.32(\mathrm{~m}, 1 \mathrm{H}), 2.27-2.18(\mathrm{~m}, 1 \mathrm{H}), 1.89-1.80(\mathrm{~m}, 2 \mathrm{H}), 1.73-1.38(\mathrm{~m}$, $6 \mathrm{H}), 1.32-1.19(\mathrm{~m}, 8 \mathrm{H}), 0.86(\mathrm{t}, J=6.8 \mathrm{~Hz}, 3 \mathrm{H}) ;{ }^{13} \mathrm{C}\left(\mathrm{CDCl}_{3}\right) \delta$ 172.0, 72.5, 54.1, 42.6, 
38.9, 31.7, 30.8, 29.7, 29.2, 25.2, 22.5, 20.1, 14.0; IR (neat) 3407, 3289, 3215, 2928, 2861, 1651, 1462, 1351, $1086 \mathrm{~cm}^{-1}$; HRMS (FAB, M+H') calcd. 228.1964 for $\mathrm{C}_{13} \mathrm{H}_{26} \mathrm{NO}_{2}$, found 228.1967 .

Lactam 6a (from epoxide 2e): ${ }^{1} \mathrm{H}$ NMR $\left(\mathrm{CDCl}_{3}\right) \delta 7.35(\mathrm{~s}, 1 \mathrm{H}), 3.84-$ $1.54-1.48(\mathrm{~m}, 2 \mathrm{H}), 1.45-1.36(\mathrm{~m}, 2 \mathrm{H}), 1.35-1.22(\mathrm{~m}, 8 \mathrm{H}), 0.86(\mathrm{t}, J=7.2 \mathrm{~Hz}, 3 \mathrm{H}) ;{ }^{13} \mathrm{C}$ $\left(\mathrm{CDCl}_{3}\right) \delta 172.8,68.0,49.2,42.7,37.3,31.8,29.2,29.0,25.8,22.6,19.4,14.0 ;$ IR (neat) $3327,3233,2929,2854,1651,1470 \mathrm{~cm}-1$; HRMS $\left(\mathrm{FAB}, \mathrm{M}+\mathrm{H}^{+}\right)$calcd. 228.1964 for $\mathrm{C}_{13} \mathrm{H}_{26} \mathrm{NO}_{2}$, found 228.1959.

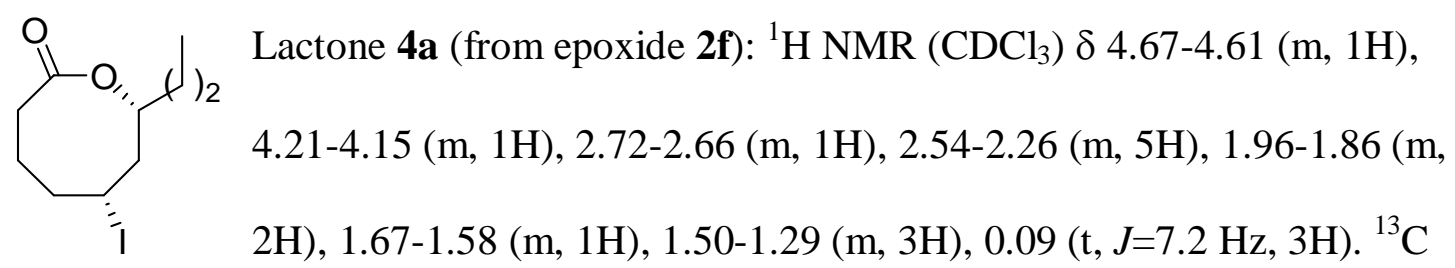
$\operatorname{NMR}\left(\mathrm{CDCl}_{3}\right) \delta 176.0,80.1,65.8,51.5,41.6,37.1,31.8,28.6,26.0,18.5,13.6 ;$ IR (neat) 2960, 2870, 1711, 1357, 1252, 1056, $1008 \mathrm{~cm}^{-1}$; HRMS (FAB, M+H' ${ }^{+}$) calcd. 297.0352 for $\mathrm{C}_{10} \mathrm{H}_{18} \mathrm{IO}_{2}$, found 297.0349; $[\alpha]_{\mathrm{D}}^{25}=48.83^{\circ}\left(\mathrm{c}=0.06, \mathrm{CHCl}_{3}\right)$

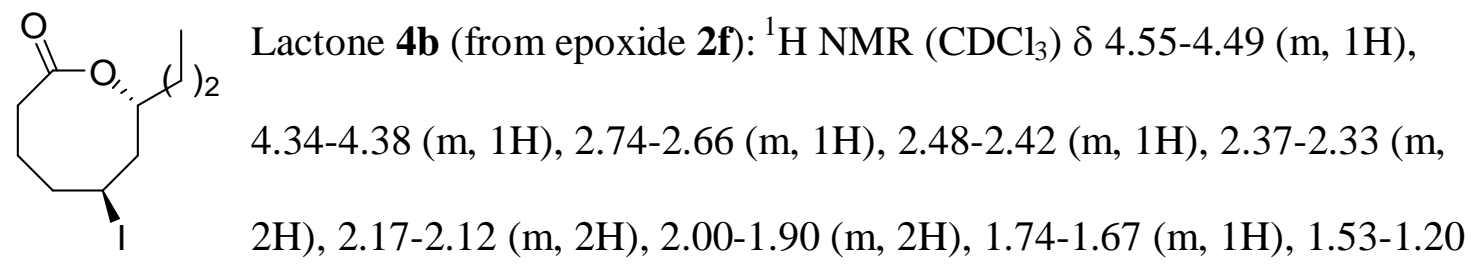

(m, 3H), $0.92(\mathrm{t}, J=8.0 \mathrm{~Hz}, 3 \mathrm{H}) .{ }^{13} \mathrm{C} \mathrm{NMR}\left(\mathrm{CDCl}_{3}\right) \delta$ 175.1, 78.3, 48.9, 37.1, 37.0, 31.2, 28.2, 27.8, 18.6, 13.7; IR (neat) 2958, 2871, 1727, 1459, 1242, 1120, $1023 \mathrm{~cm}^{-1}$; HRMS 
$\left(\mathrm{FAB}, \mathrm{M}+\mathrm{H}^{+}\right)$calcd. 297.0352 for $\mathrm{C}_{10} \mathrm{H}_{18} \mathrm{IO}_{2}$, found $297.0348 ;[\alpha]_{\mathrm{D}}{ }^{25}=-6.95^{\circ}(\mathrm{c}=0.03$, $\left.\mathrm{CHCl}_{3}\right)$.

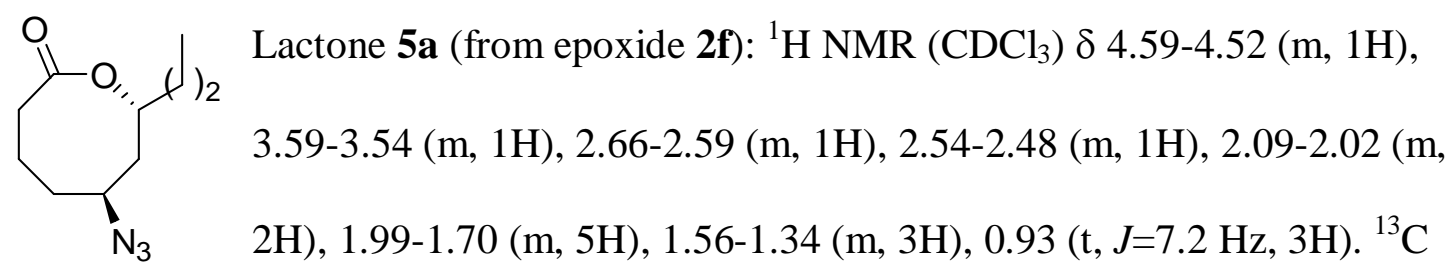
$\operatorname{NMR}\left(\mathrm{CDCl}_{3}\right) \delta 175.0,75.6,58.7,41.4,37.3,32.3,32.1,24.5,18.7,13.7 ;$ IR (neat) 2960, 2935, 2873, 2101, 1728, 1460, 1253, 1200, 1144, $1030 \mathrm{~cm}^{-1}$; HRMS (FAB, M+H ${ }^{+}$) calcd. 212.1399 for $\mathrm{C}_{10} \mathrm{H}_{18} \mathrm{~N}_{3} \mathrm{O}_{2}$, found 212.1378; $[\alpha]_{\mathrm{D}}{ }^{25}=-18.42^{\circ}\left(\mathrm{c}=0.02, \mathrm{CHCl}_{3}\right)$.

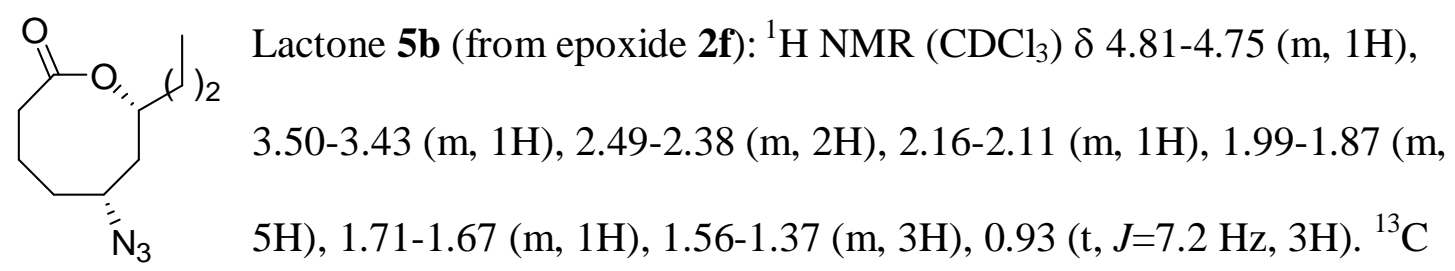
$\operatorname{NMR}\left(\mathrm{CDCl}_{3}\right) \delta 176.1,76.9,61.0,43.1,37.4,33.8,32.8,26.4,18.6,15.3,13.7 ;$ IR (neat) 2959, 2874, 1738, 1456, 1249, $1153 \mathrm{~cm}^{-1}$; HRMS (FAB, M+H') calcd. 212.1399 for $\mathrm{C}_{10} \mathrm{H}_{18} \mathrm{~N}_{3} \mathrm{O}_{2}$, found 212.1391; $[\alpha]_{\mathrm{D}}^{25}=29.49^{\circ}\left(\mathrm{c}=0.01, \mathrm{CHCl}_{3}\right)$.

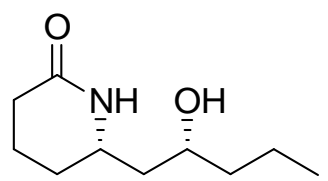
Lactam 6a (from epoxide 2f): ${ }^{1} \mathrm{H}$ NMR $\left(\mathrm{CDCl}_{3}\right) \delta 7.72(\mathrm{~s}, 1 \mathrm{H}), 4.06$ (m, 1H), 3.81-3.75 (m, 1H), 3.55-3.45 (m, 1H), 2.40-2.18 (m, 2H), $1.89-1.81(\mathrm{~m}, 2 \mathrm{H}), 1.75-1.20(\mathrm{~m}, 8 \mathrm{H}), 0.89(\mathrm{t}, J=9.6 \mathrm{~Hz}, 3 \mathrm{H}) ;{ }^{13} \mathrm{C} \mathrm{NMR}\left(\mathrm{CDCl}_{3}\right) \delta 172.2$, 72.3, 54.2, 42.5, 41.1, 30.7, 29.6, 20.0, 18.5, 14.1; IR (neat) 3318, 2955, 2931, 2871, 1645, $1467 \mathrm{~cm}^{-1}$; HRMS (FAB, $\mathrm{M}+\mathrm{H}^{+}$) calcd. 186.1494 for $\mathrm{C}_{10} \mathrm{H}_{20} \mathrm{NO}_{2}$, found 186.1495. 


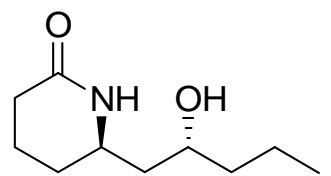

Lactam 6b (from epoxide 2f): ${ }^{1} \mathrm{H}$ NMR $\left(\mathrm{CDCl}_{3}\right) \delta 7.44(\mathrm{~s}, 1 \mathrm{H}), 3.96$ (s, $1 \mathrm{H}), 3.88-3.82(\mathrm{~s}, 1 \mathrm{H}), 3.73-3.68(\mathrm{~m}, 1 \mathrm{H}), 2.39-2.22(\mathrm{~m}, 2 \mathrm{H})$, 1.89-1.80 (m, 2H), 1.76-1.61 (m, 2H), 1.55-1.32 (m, 6H), $0.92(\mathrm{t}, J=3.6,3 \mathrm{H}),{ }^{13} \mathrm{C}$ NMR $\left(\mathrm{CDCl}_{3}\right) \delta$ 172.9, 67.8, 49.3, 42.6, 39.4, 31.0, 29.0, 19.4, 19.0, 14.0; IR (neat) 3302, 2953, 2871, 1655, 1467, 1411, 1351, $1308 \mathrm{~cm}^{-1}$; HRMS (FAB) $\mathrm{m} / z\left(\mathrm{M}+\mathrm{H}^{+}\right)$calcd. 186.1494 for $\mathrm{C}_{10} \mathrm{H}_{20} \mathrm{NO}_{2}^{+}$, found 186.1496 .

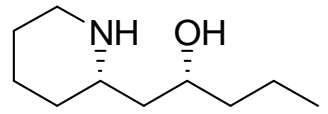

(-)-epihalosaline (8a): A $10 \mathrm{~mL}$ flask was charged with lactam $\mathbf{6 a}$ (from epoxide 2f) $(0.027 \mathrm{~g}, 0.15 \mathrm{mmol})$ and dissolved in $1 \mathrm{~mL}$ of anhydrous ether. To this solution, lithium aluminum hydride $(0.028 \mathrm{~g}, 0.75 \mathrm{mmol})$ was added in one portion. After $2 \mathrm{hr}$ the reaction mixture was quenched with $3 \mathrm{~mL}$ of a $10 \%$ $\mathrm{NaOH}$ solution, and then extracted with ethyl acetate $(3 \times 25 \mathrm{~mL})$. The combined organics were dried over $\mathrm{MgSO}_{4}$ and concentrated in vacuo. The crude product was purified via column chromatography $\left(91 \% \mathrm{CH}_{2} \mathrm{Cl}_{2}, 7.6 \% \mathrm{MeOH}, 1.4 \% \mathrm{NH}_{4} \mathrm{OH}\right)$ to afford (-)epihalosaline (8a) $(0.019 \mathrm{~g}, 76 \%)$ as a colorless oil. ${ }^{1} \mathrm{H}$ NMR $\left(\mathrm{CDCl}_{3}\right) \delta 3.85-3.79(\mathrm{~m}$, 1H), $3.66(\mathrm{~s}, 1 \mathrm{H}), 3.08-3.03(\mathrm{~m}, 1 \mathrm{H}), 2.77-2.70(\mathrm{~m}, 1 \mathrm{H}), 2.64-2.56(\mathrm{~m}, 1 \mathrm{H}), 1.84-1.79(\mathrm{~m}$, 1H), 1.66-1.08 (m, 9H), $0.91(\mathrm{t}, J=6.8 \mathrm{~Hz}, 3 \mathrm{H}) ;{ }^{13} \mathrm{C} \mathrm{NMR}\left(\mathrm{CDCl}_{3}\right) \delta 72.7,58.3,45.9$, 42.3, 40.4, 34.1, 27.0, 24.4, 18.6, 14.2; IR (neat) 3309, 2929, 2856, 1456, $1123 \mathrm{~cm}^{-1}$; HRMS (FAB) $m / z\left(\mathrm{M}+\mathrm{H}^{+}\right)$calcd. 172.1701 for $\mathrm{C}_{10} \mathrm{H}_{22} \mathrm{NO}^{+}$, found $172.1690 ;[\alpha]_{\mathrm{D}}^{25}=$ $29.61^{\circ}\left(\mathrm{c}=0.01, \mathrm{CHCl}_{3}\right)$.

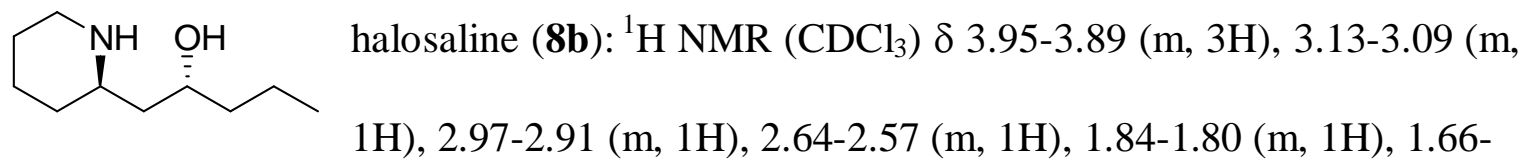


$1.25(\mathrm{~m}, 11 \mathrm{H}), 0.91(\mathrm{t}, J=7.2 \mathrm{~Hz}, 3 \mathrm{H}) ;{ }^{13} \mathrm{C} \mathrm{NMR}\left(\mathrm{CDCl}_{3}\right) \delta 68.7,54.8,46.8,41.9,40.1$, 31.3, 25.9, 24.6, 19.0, 14.2; IR (neat) 3364, 2975, 2899, 1455, 1381, 1090, 1050, $881 \mathrm{~cm}^{-}$ ${ }^{1}$; HRMS (FAB) $m / z\left(\mathrm{M}+\mathrm{H}^{+}\right)$calcd. 172.1701 for $\mathrm{C}_{10} \mathrm{H}_{22} \mathrm{NO}^{+}$, found $172.1690 ;[\alpha]_{\mathrm{D}}{ }^{25}=-$ $18.20^{\circ}\left(\mathrm{c}=0.30, \mathrm{CHCl}_{3}\right)$.

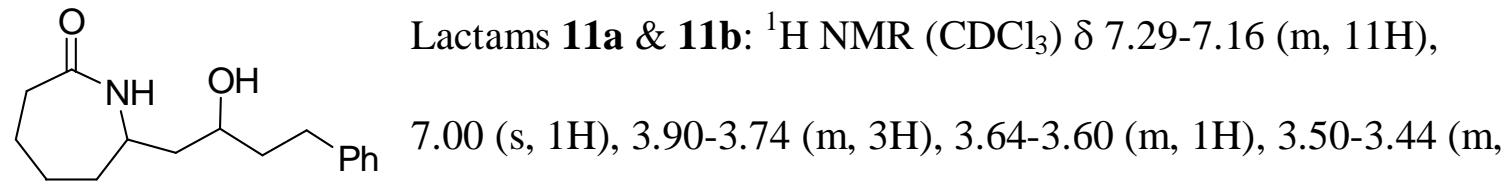
$1 \mathrm{H}), 2.83-2.62(\mathrm{~m}, 4 \mathrm{H}), 2.53-2.37(\mathrm{~m}, 4 \mathrm{H}), 1.97-1.94(\mathrm{~m}, 2 \mathrm{H}), 1.91-1.26(\mathrm{~m}, 18 \mathrm{H}) ;{ }^{13} \mathrm{C}$ $\operatorname{NMR}\left(\mathrm{CDCl}_{3}\right) \delta 178.3,178.2,141.9,128.38,128.36,128.34,125.78,125.76,69.9,67.5$, $53.5,51.2,43.0,42.2,40.2,39.5,36.6,35.5,32.0,31.7,29.7,29.6,23.1,23.0$; IR (neat) $3371,3274,3206,3079,3060,3021,2924,2855,1650,1455,1182,1095,696 \mathrm{~cm}^{-1}$; HRMS (FAB) $m / z\left(\mathrm{M}+\mathrm{H}^{+}\right)$calcd. 262.1807 for $\mathrm{C}_{16} \mathrm{H}_{24} \mathrm{NO}_{2}{ }^{+}$, found 262.1802 . 

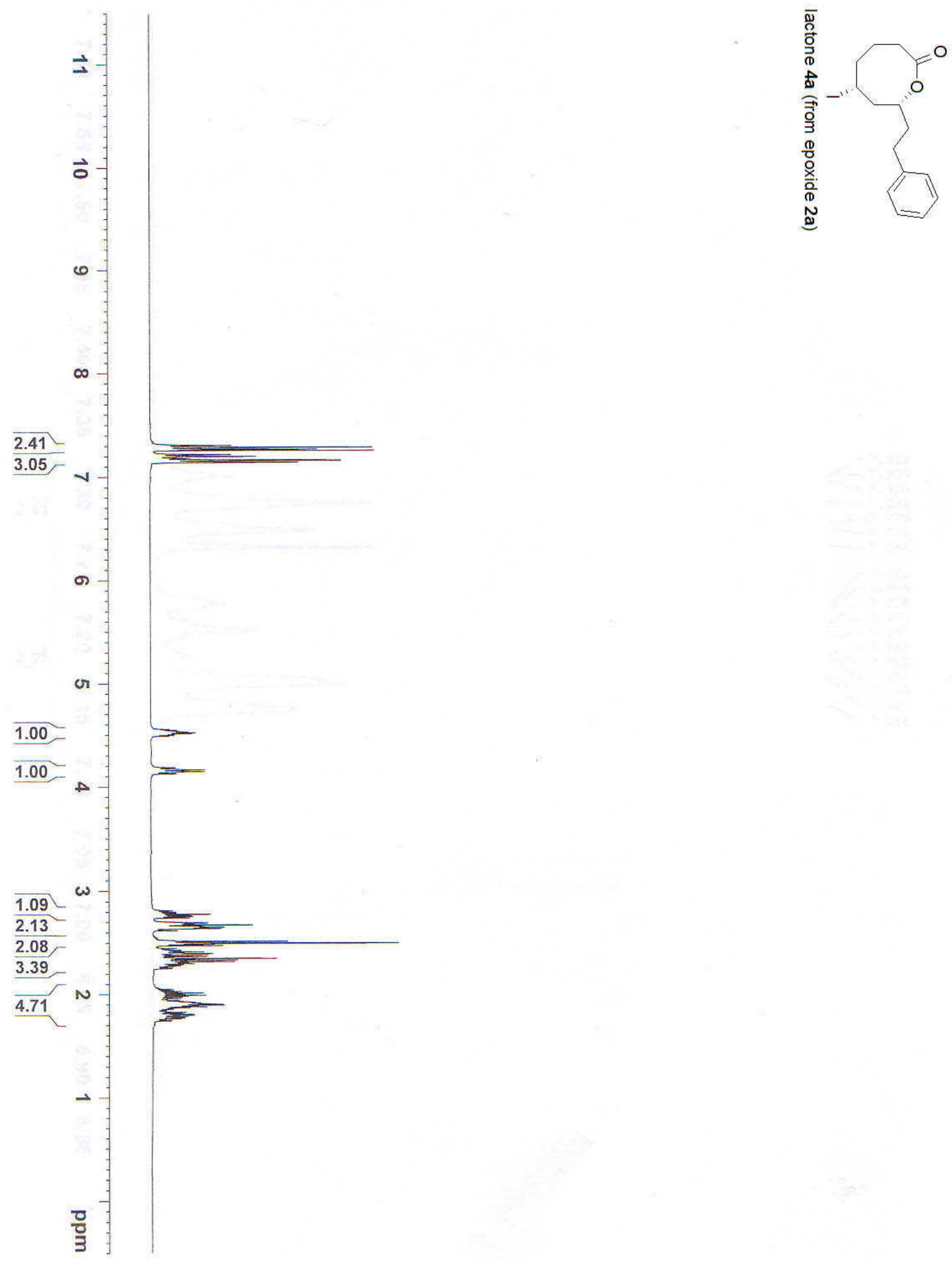

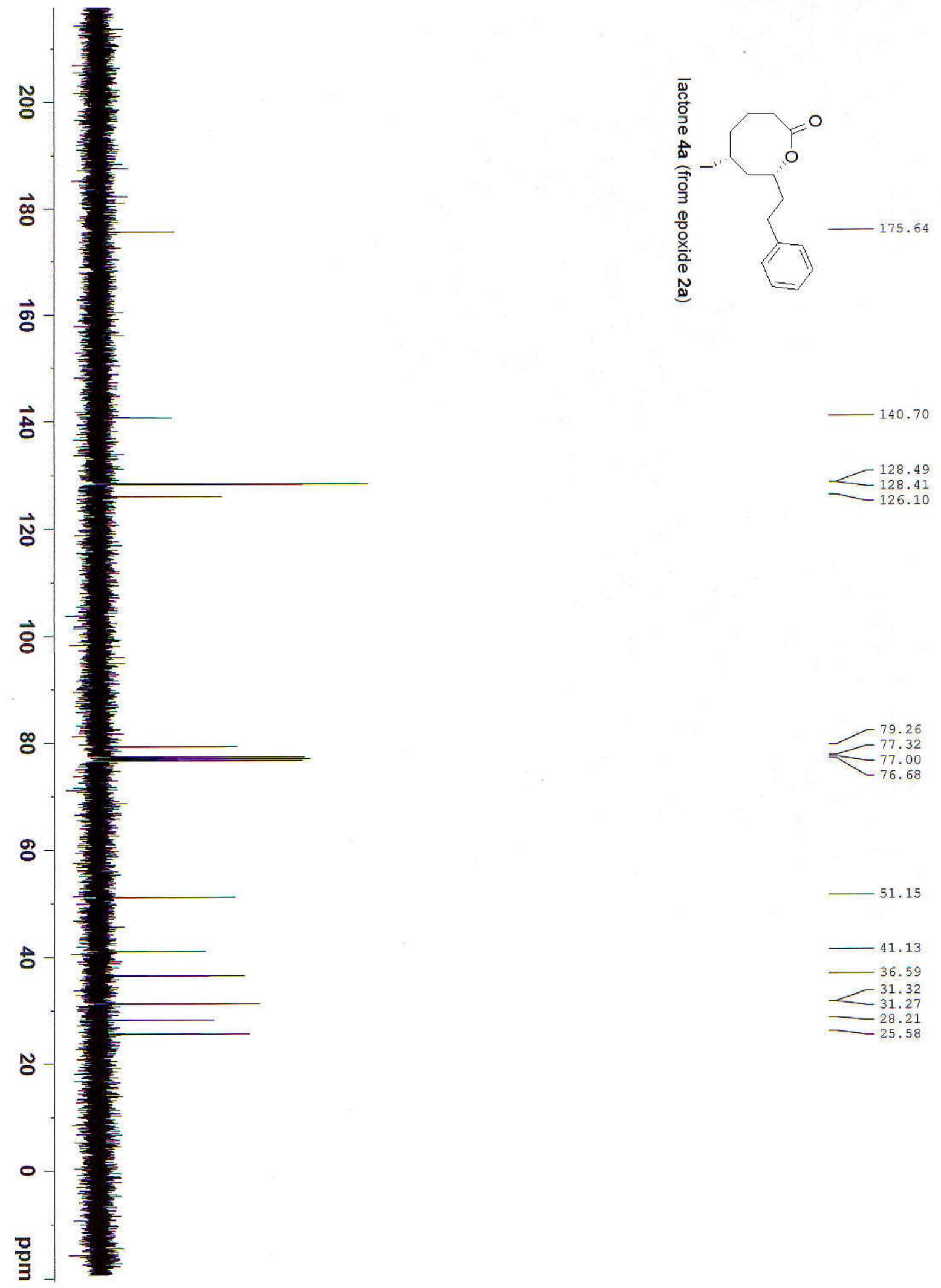

$-128.49$

$+128.41$

126.10

79.26
-77.32

77.00

$-76.68$

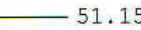

$-41.13$

$-36.59$

$\begin{array}{r}-31.27 \\ \hline\end{array}$

$-28.2$

$-25.58$ 

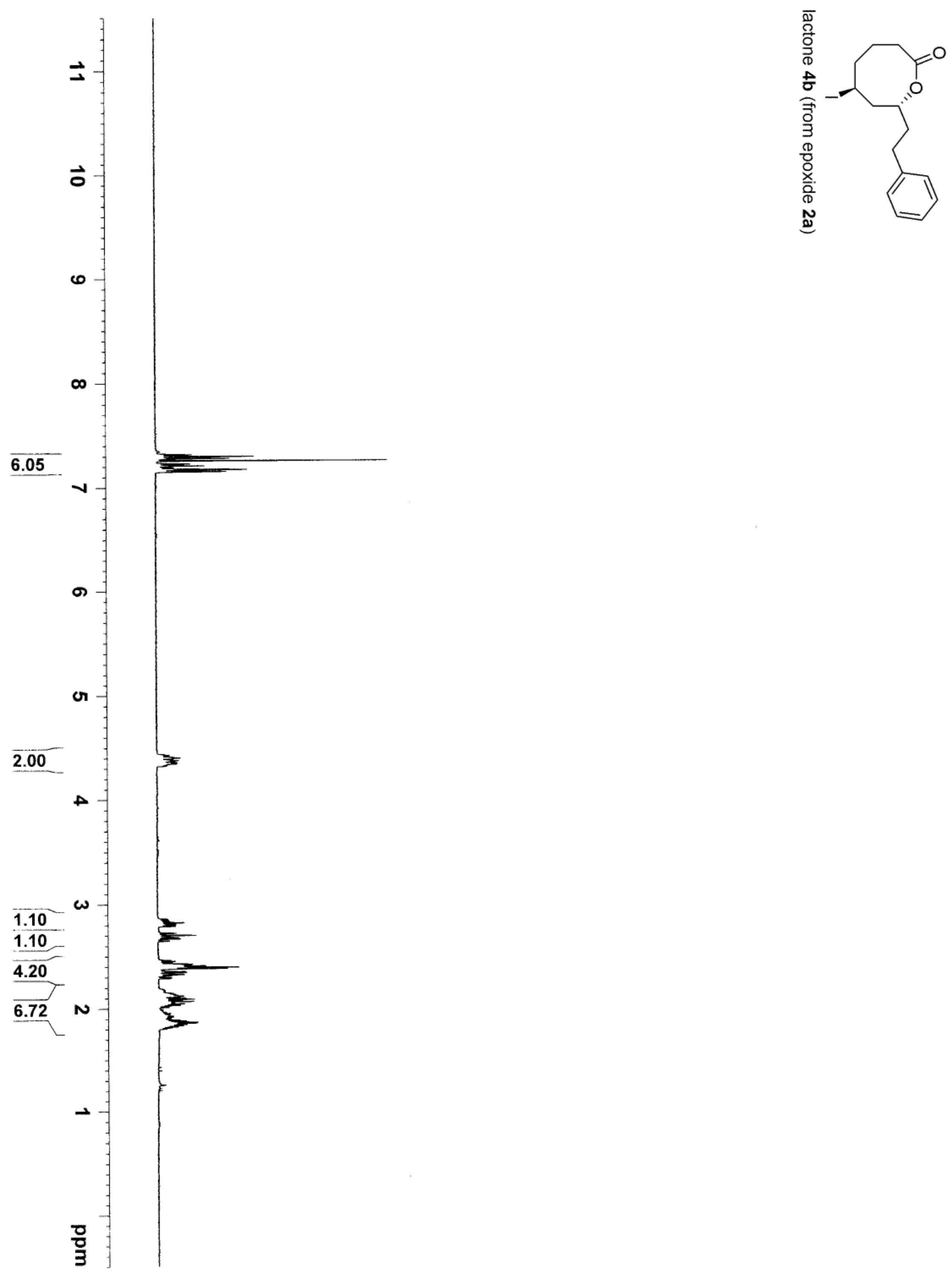


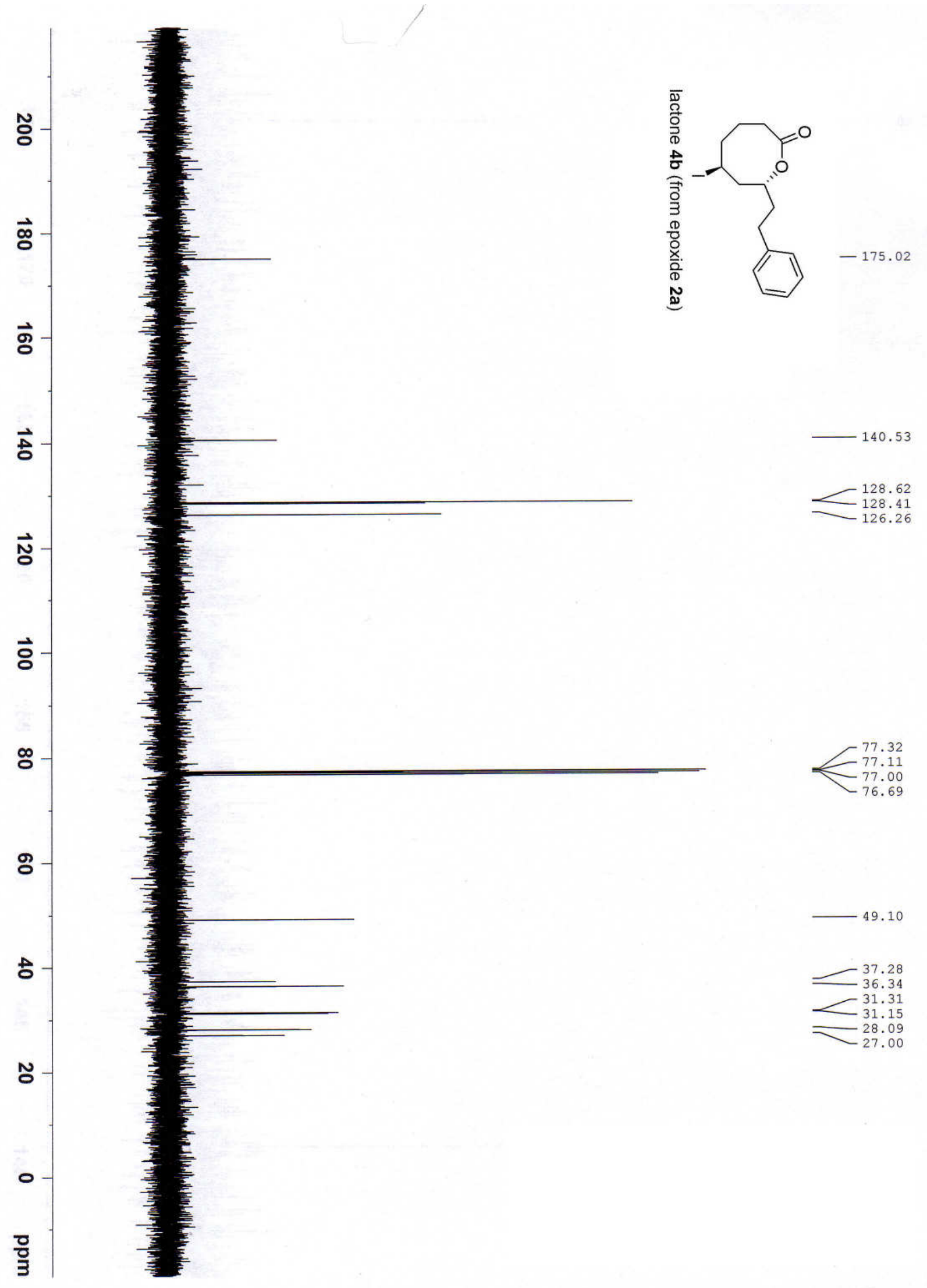



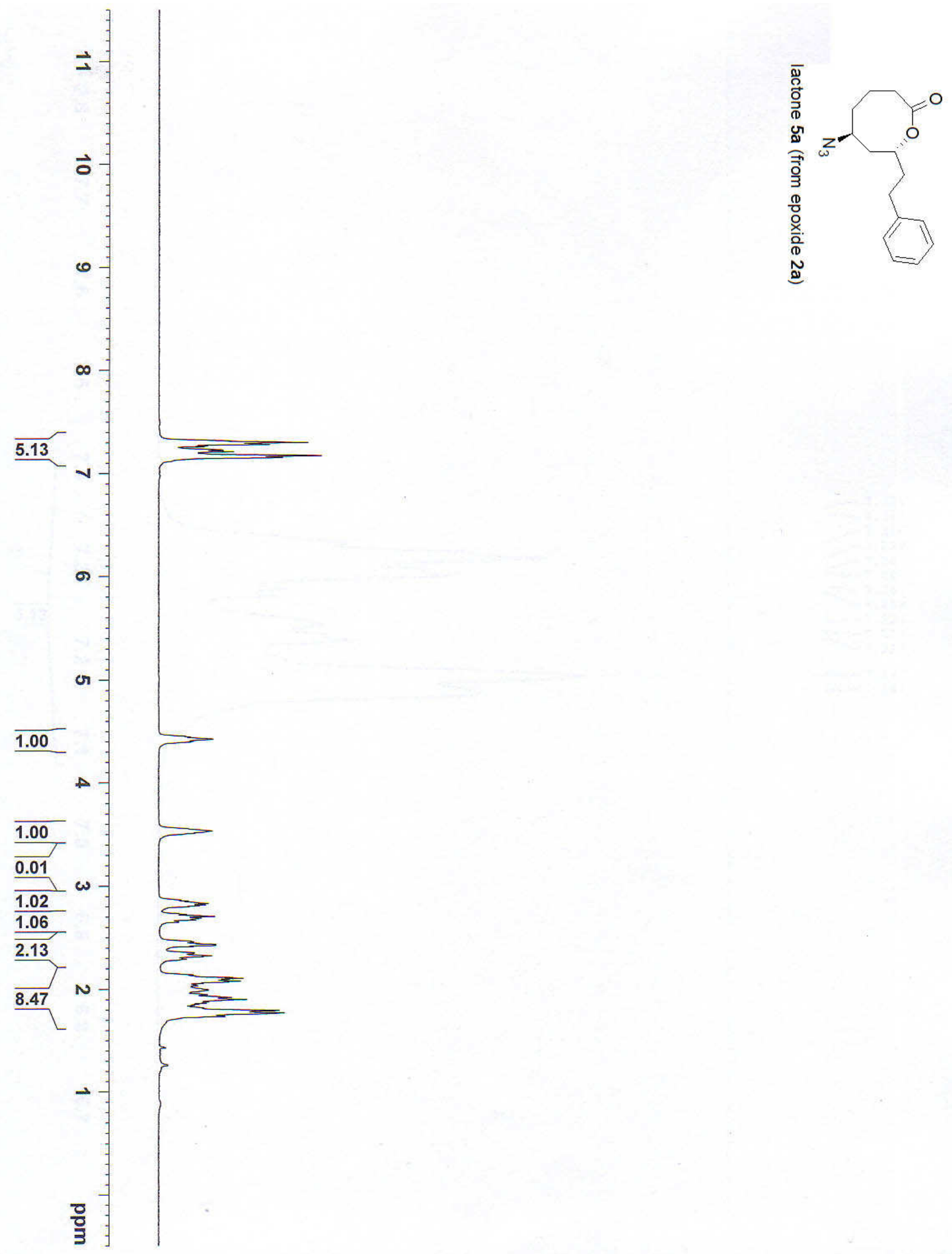

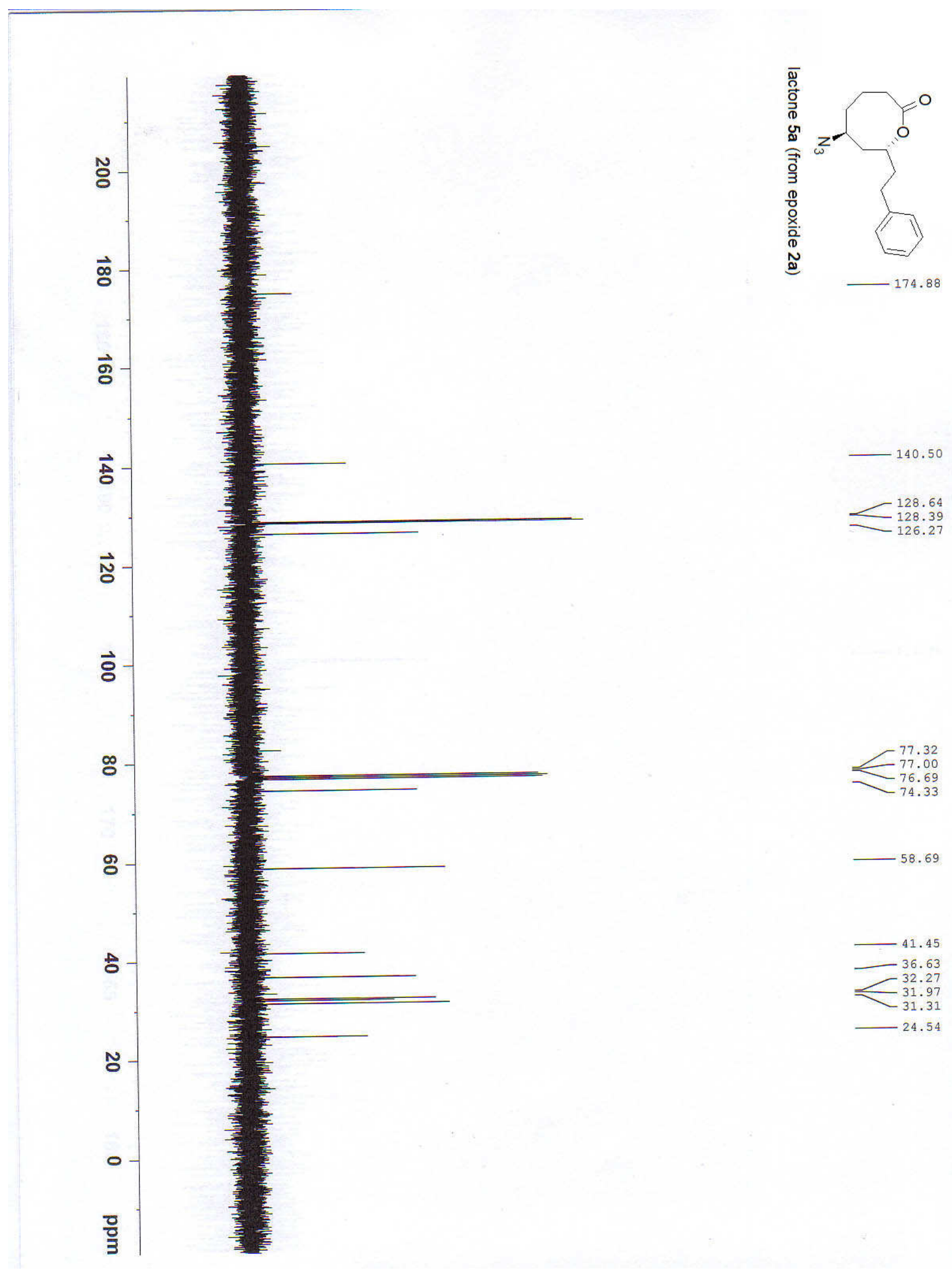

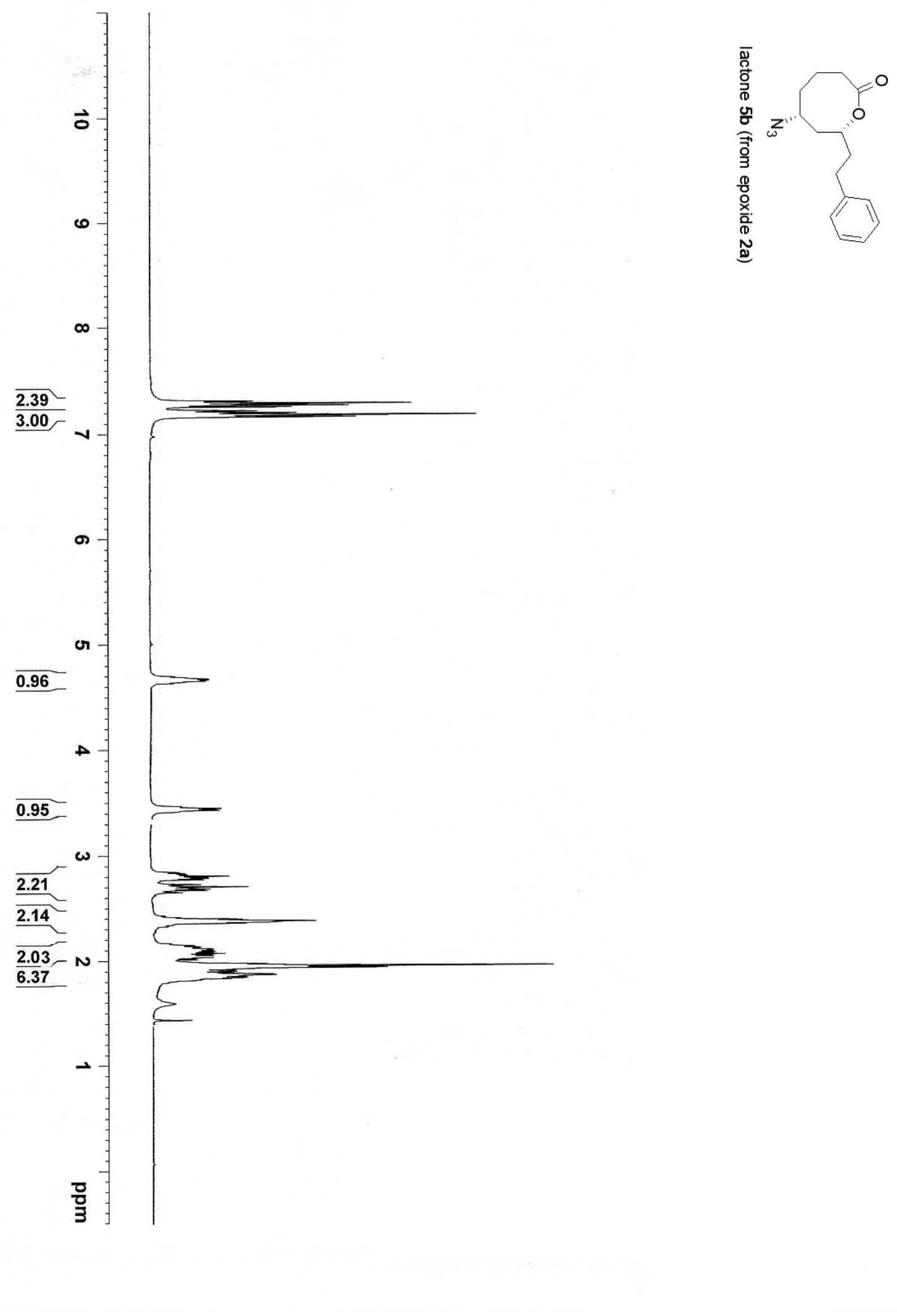

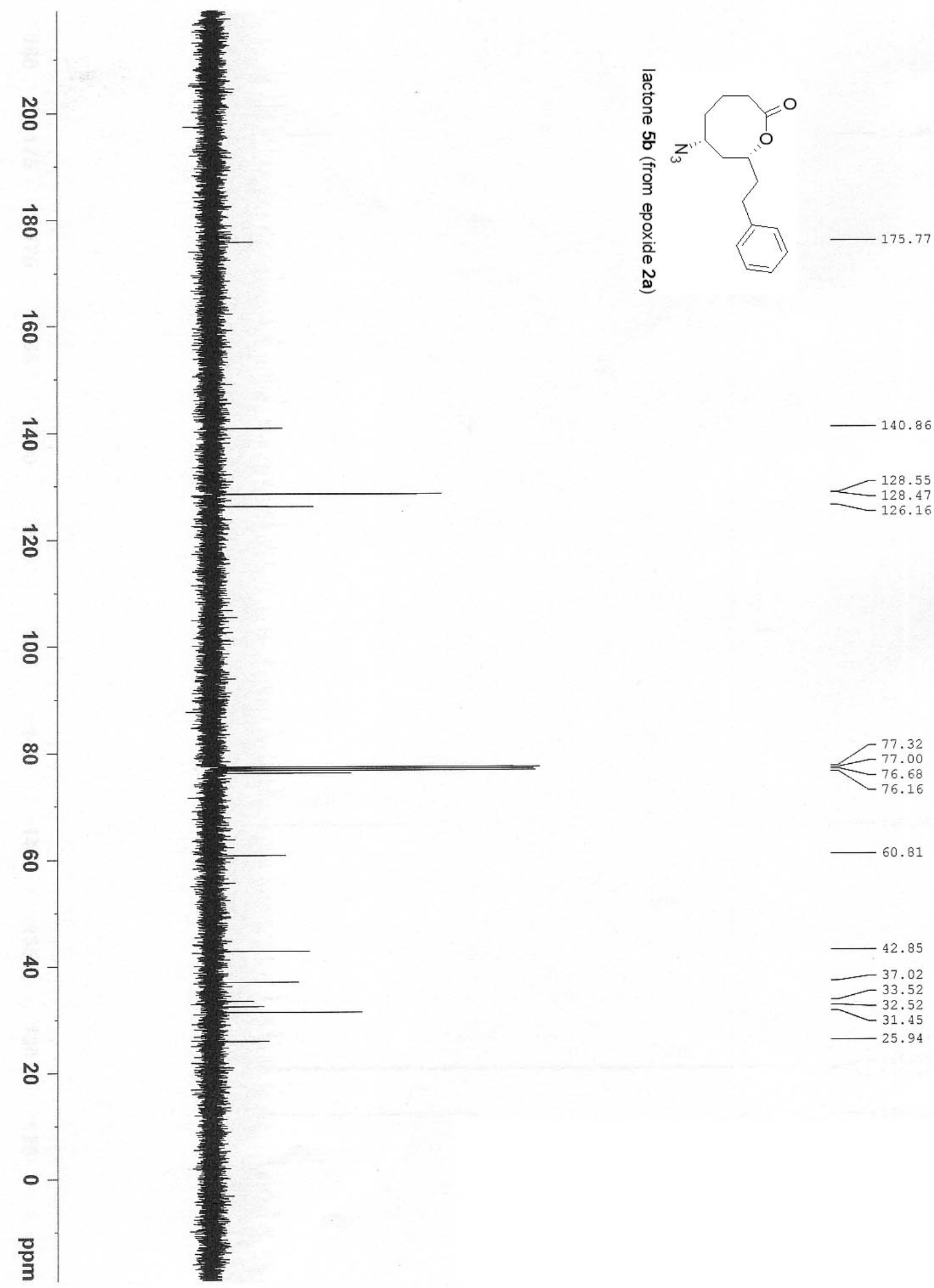

- 76.68

$-76.16$

$-60.81$

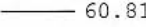

$-42.85$

37.02

$-33.52$

$\begin{array}{r}-32.52 \\ \hdashline-31.45 \\ \hline\end{array}$

$-25.94$ 


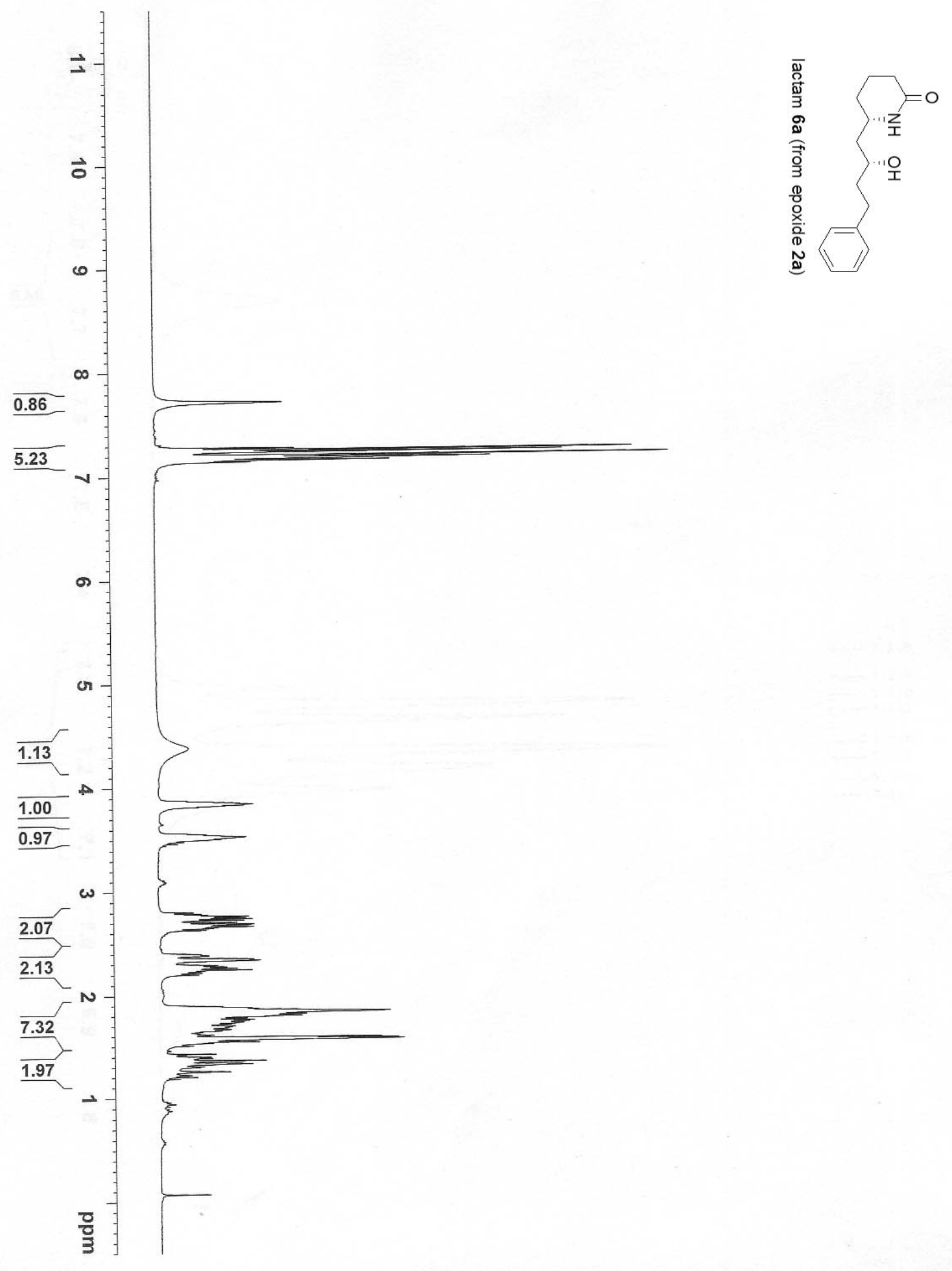




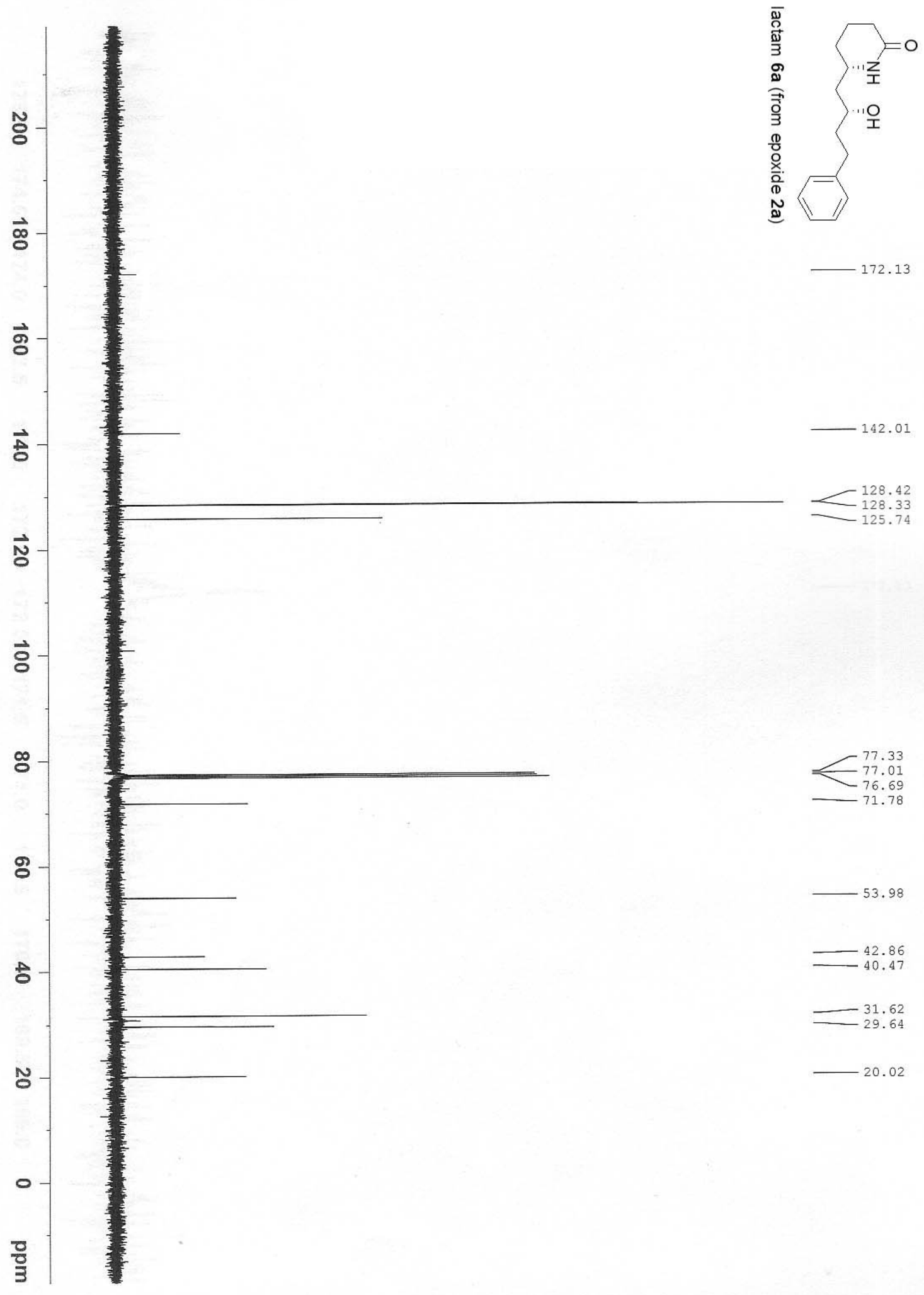




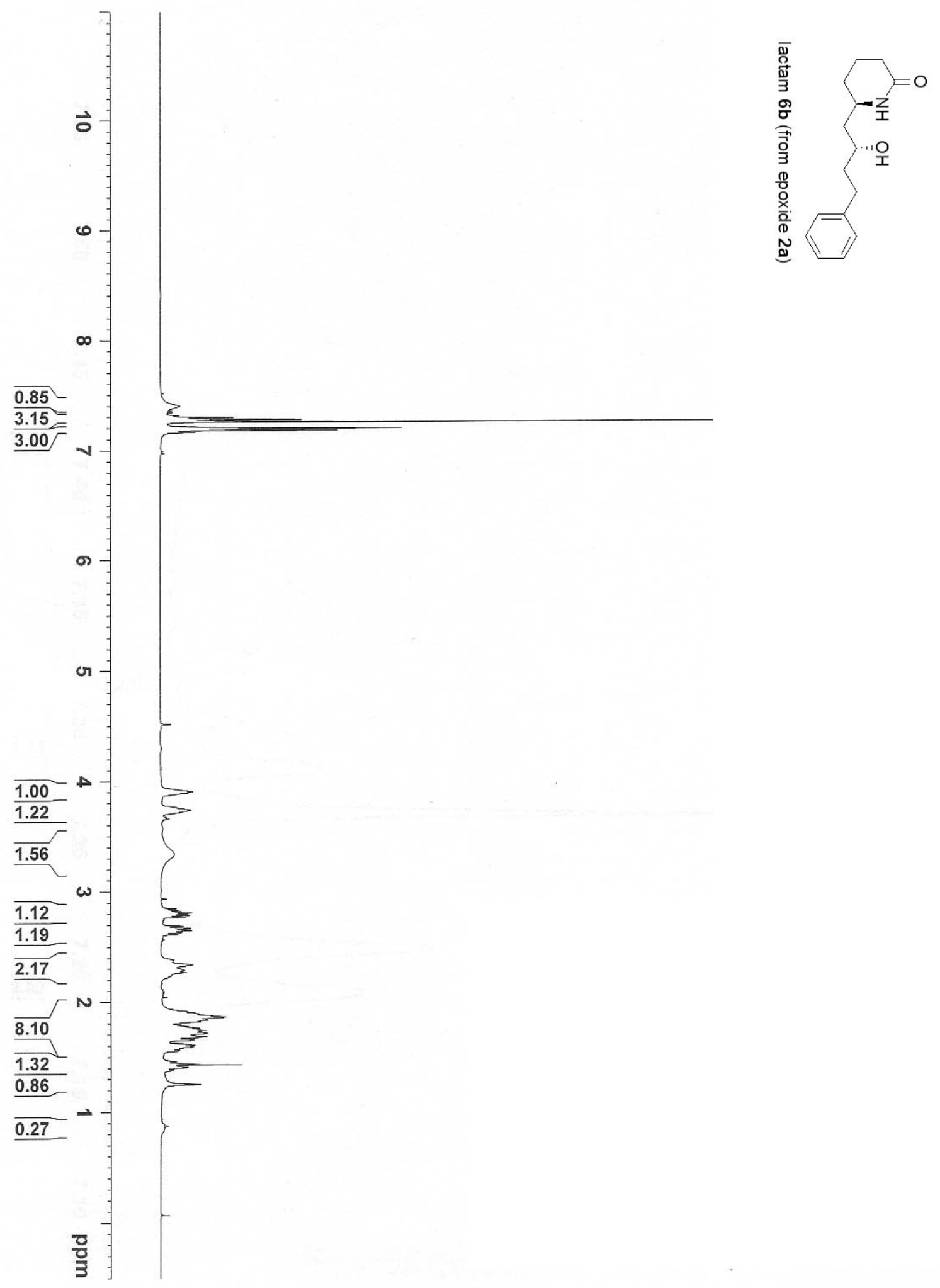




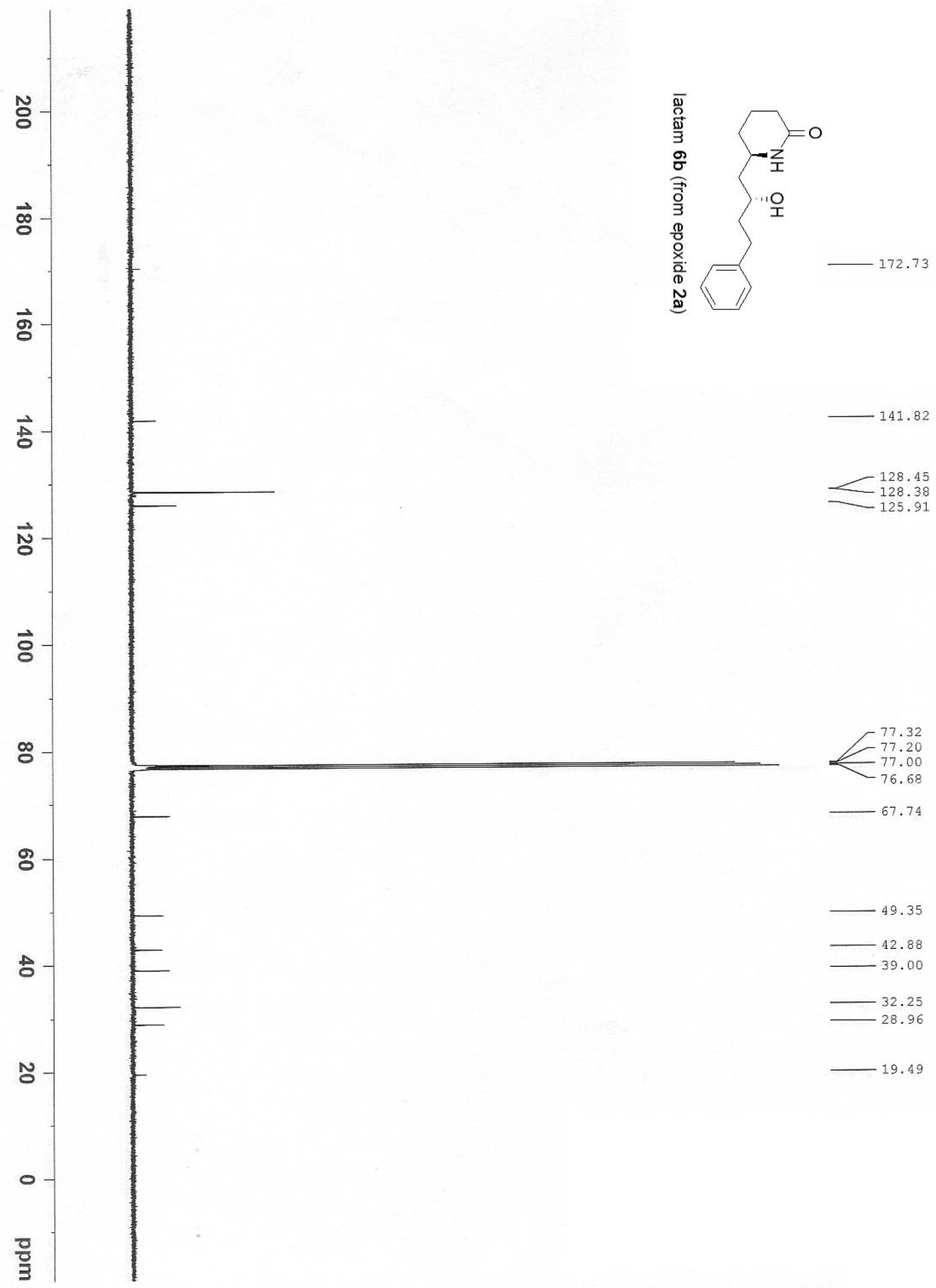




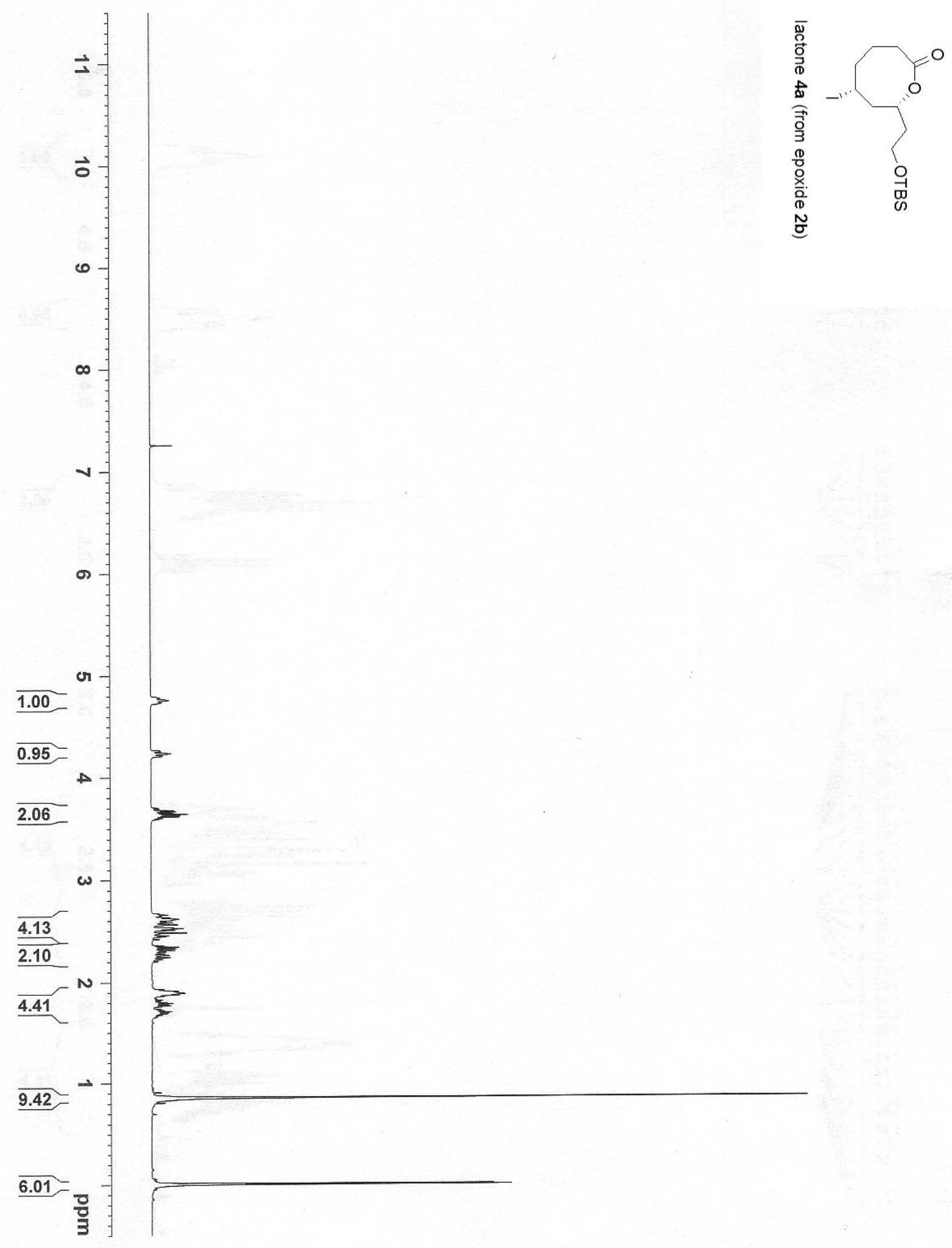



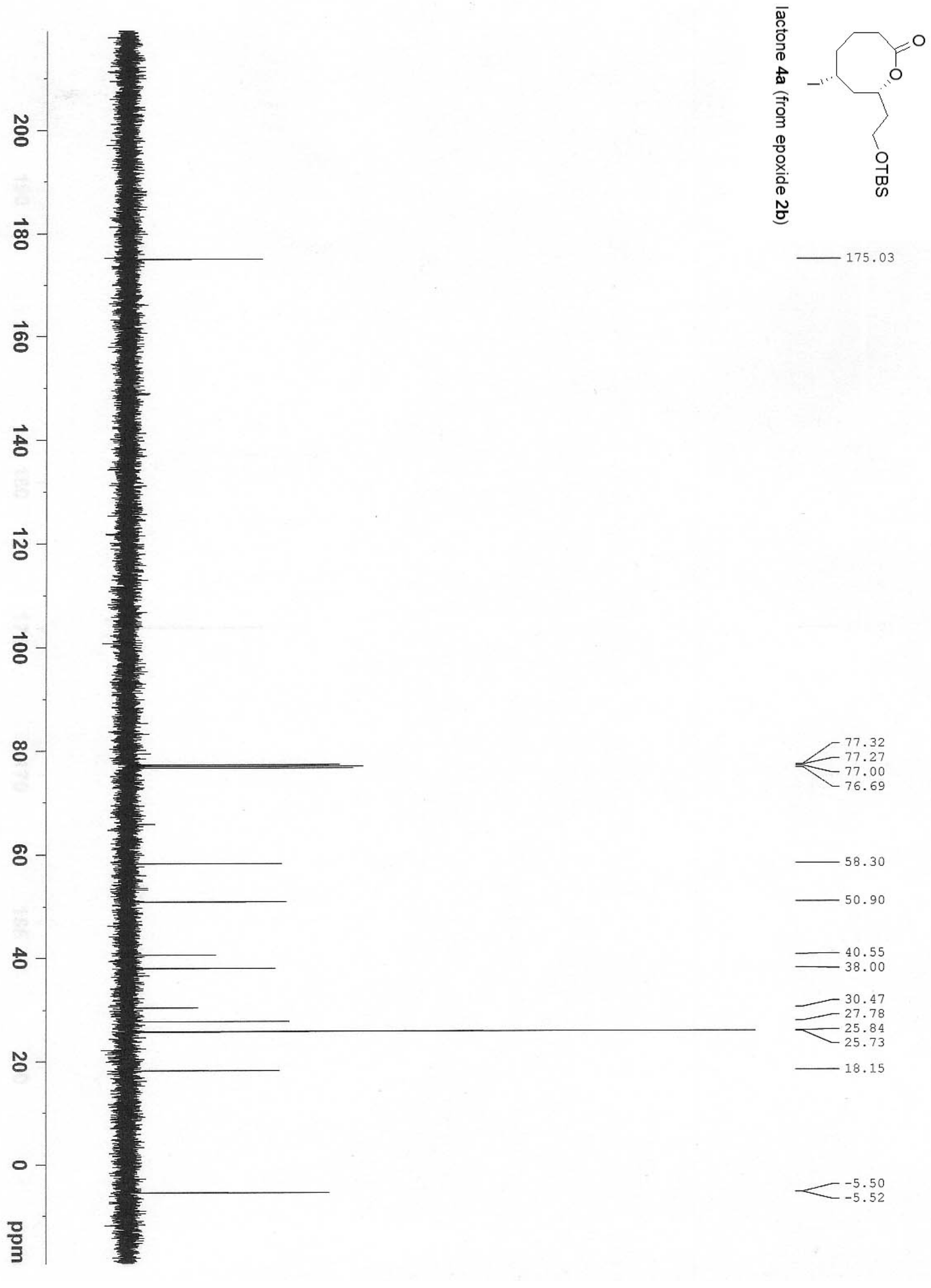


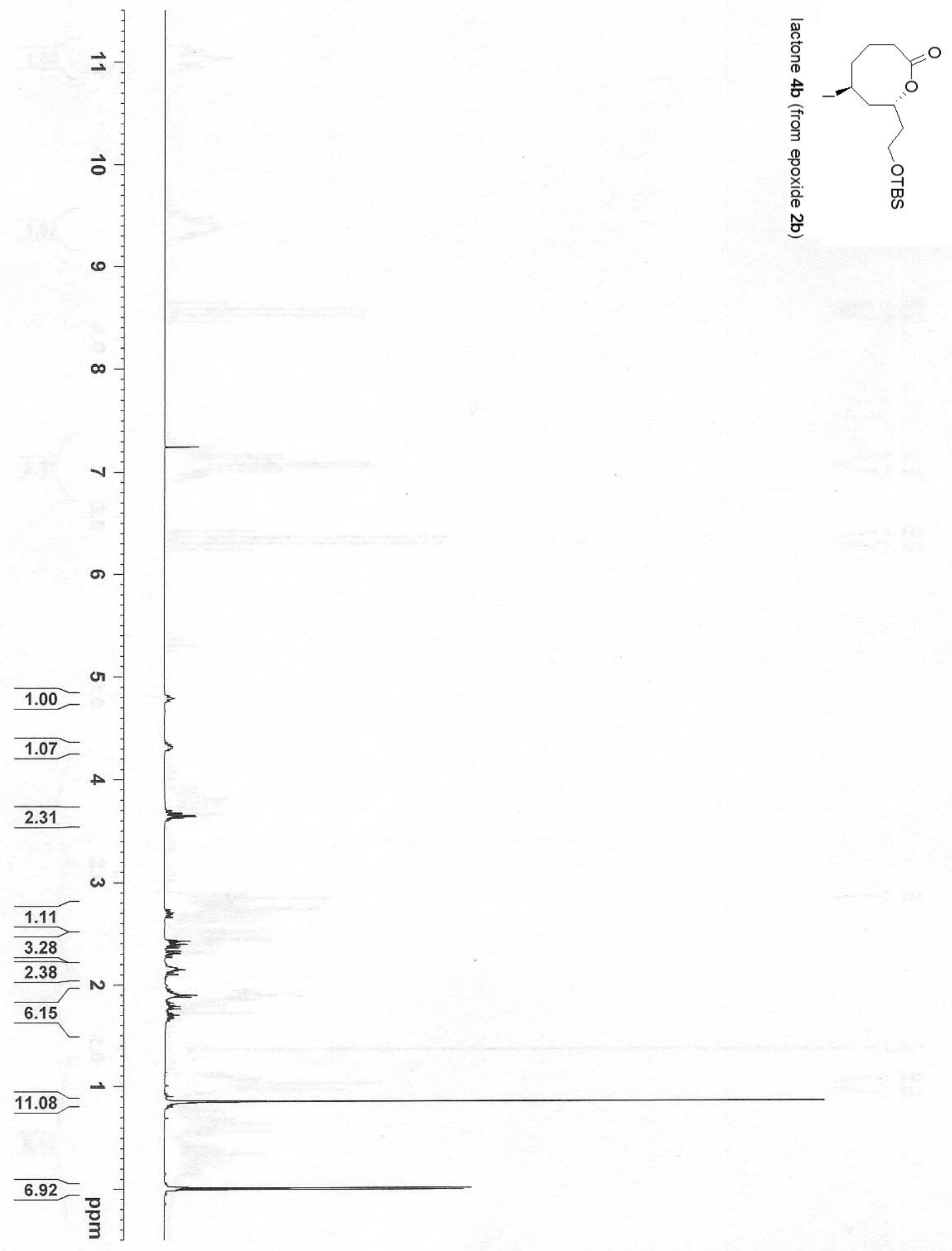



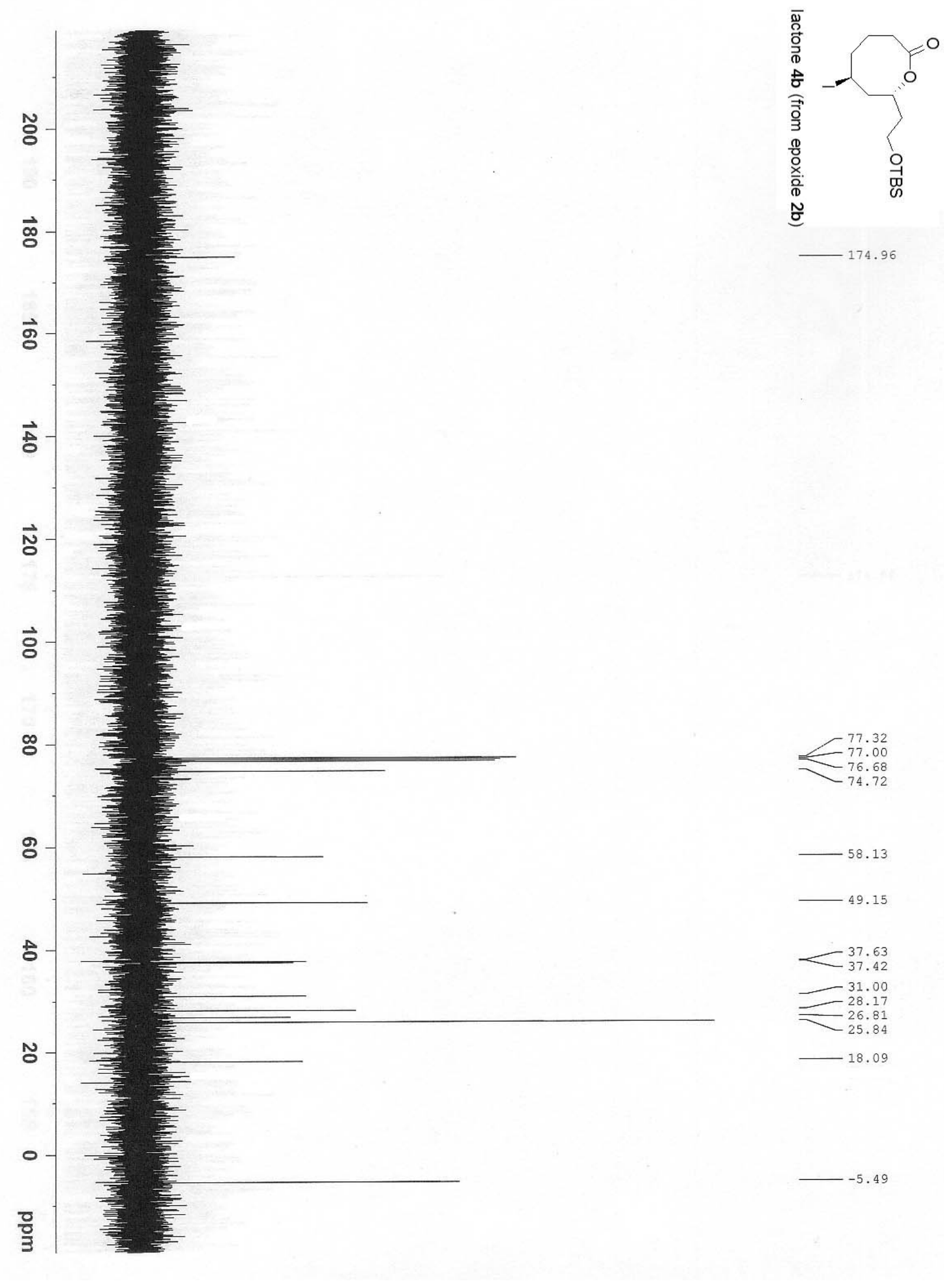

$\quad 37.63$
$-\quad 37.42$
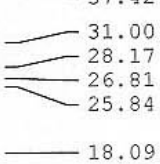


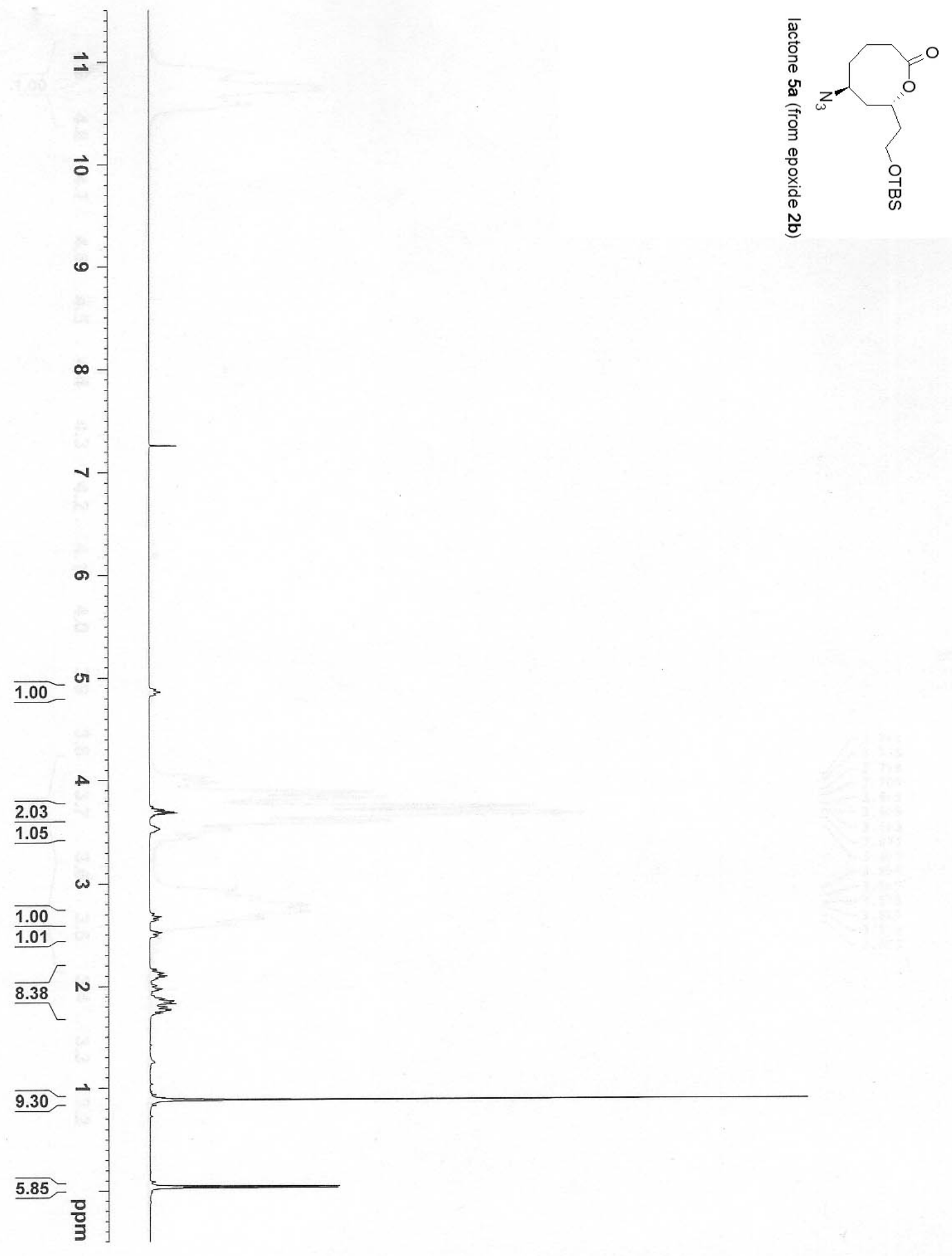




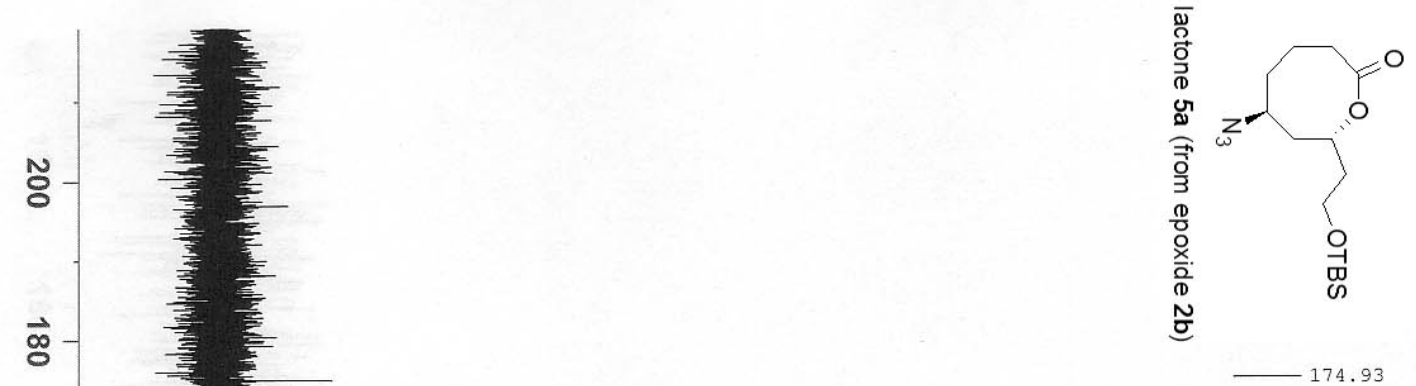

홍

$\overrightarrow{\text { t }}$

$\vec{N}$

$\overrightarrow{8}$

๕

8

है

ำ

o

总

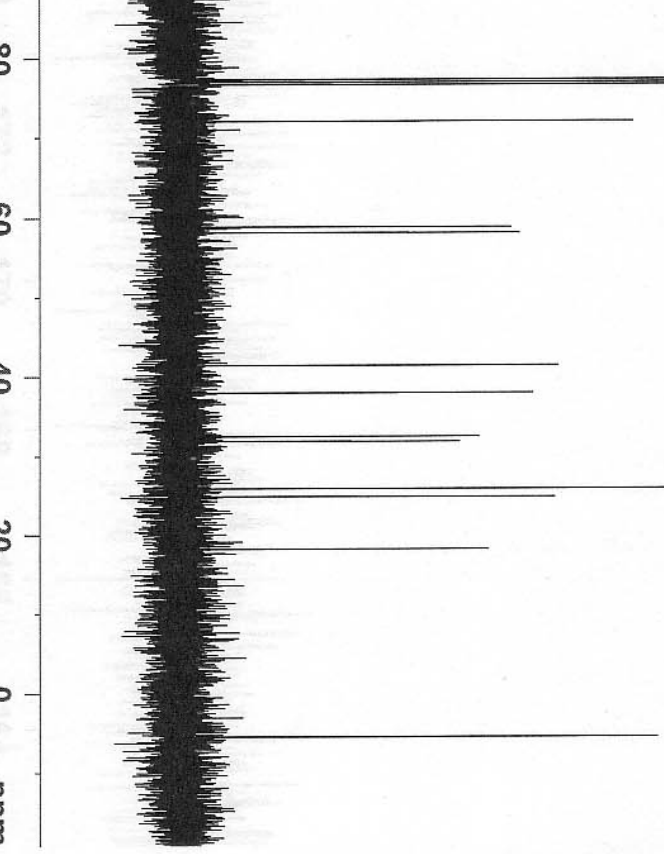

$\chi_{-5.49}^{-5.44}$ 


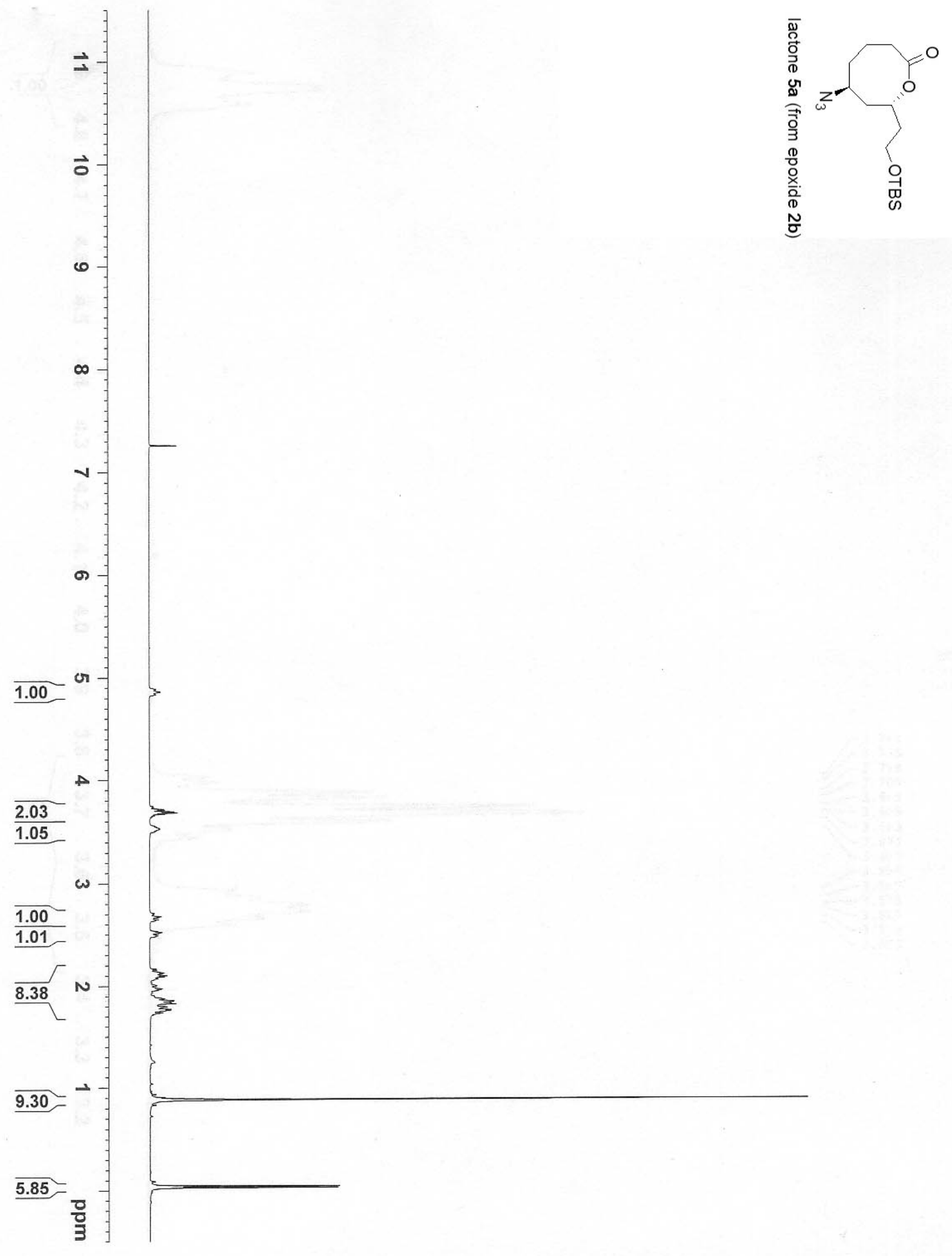



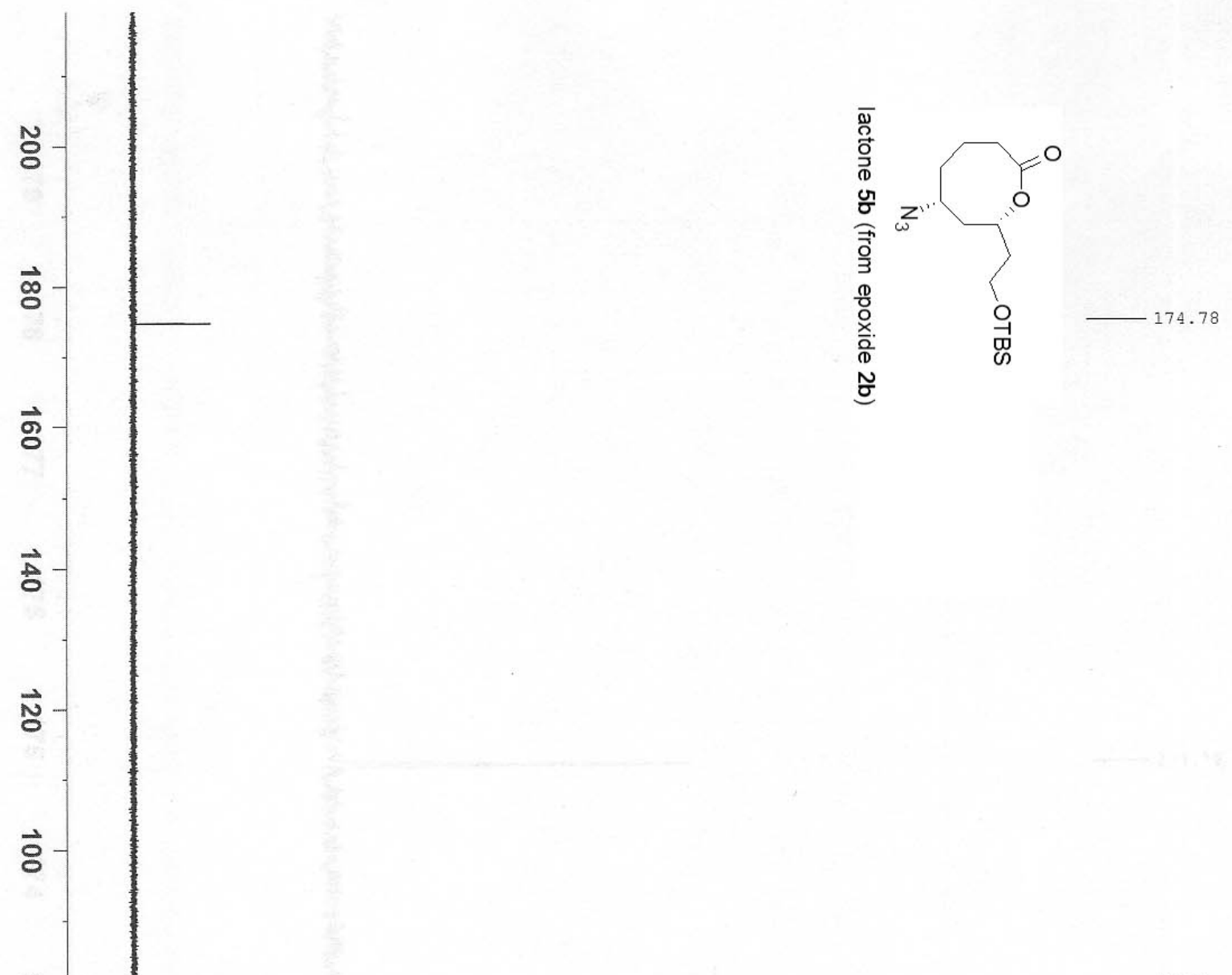

ஃ
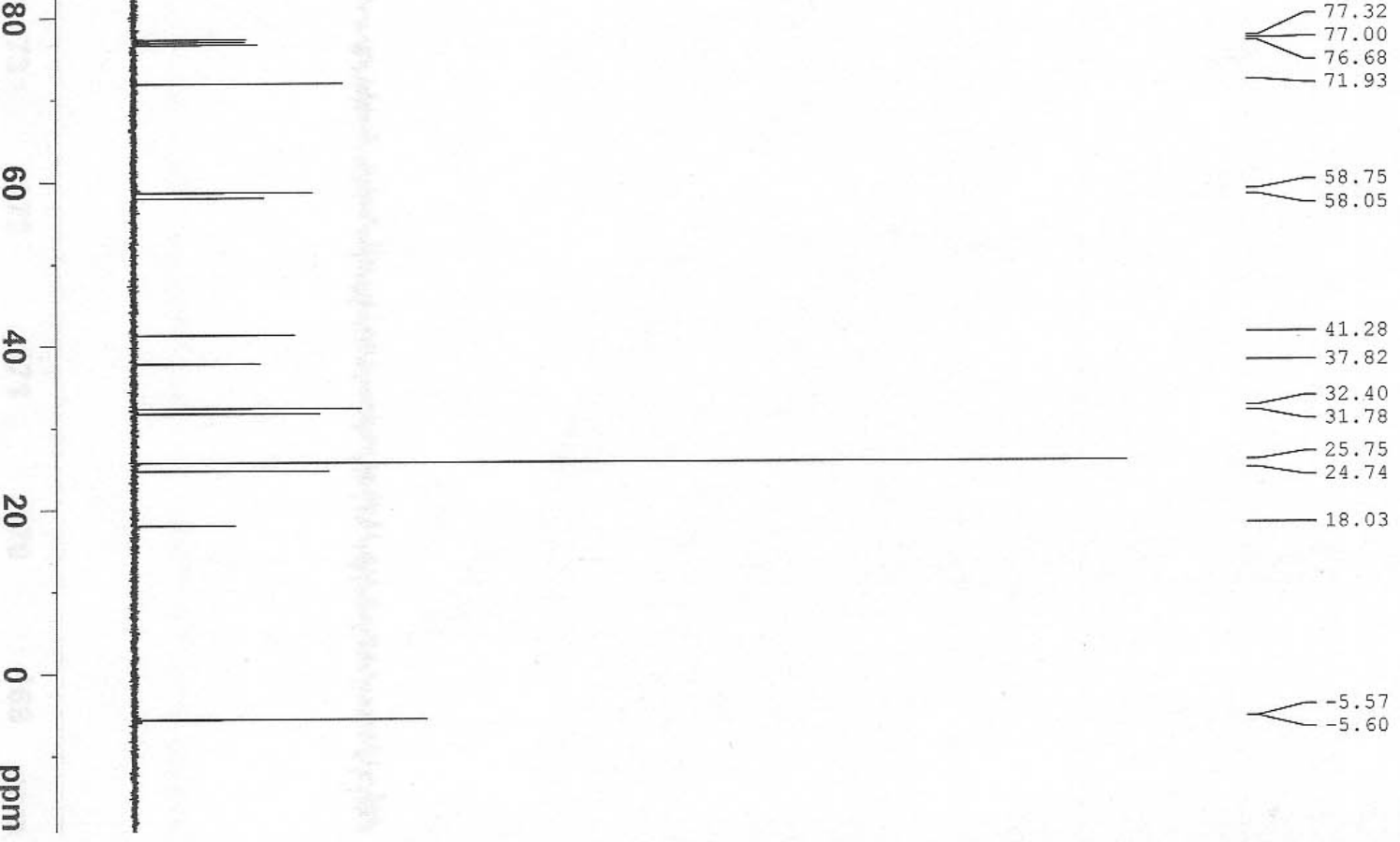

$-41.28$

- 37.82

$-32.40$

$-31.78$

$=24.74$

$-18.03$

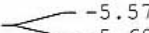




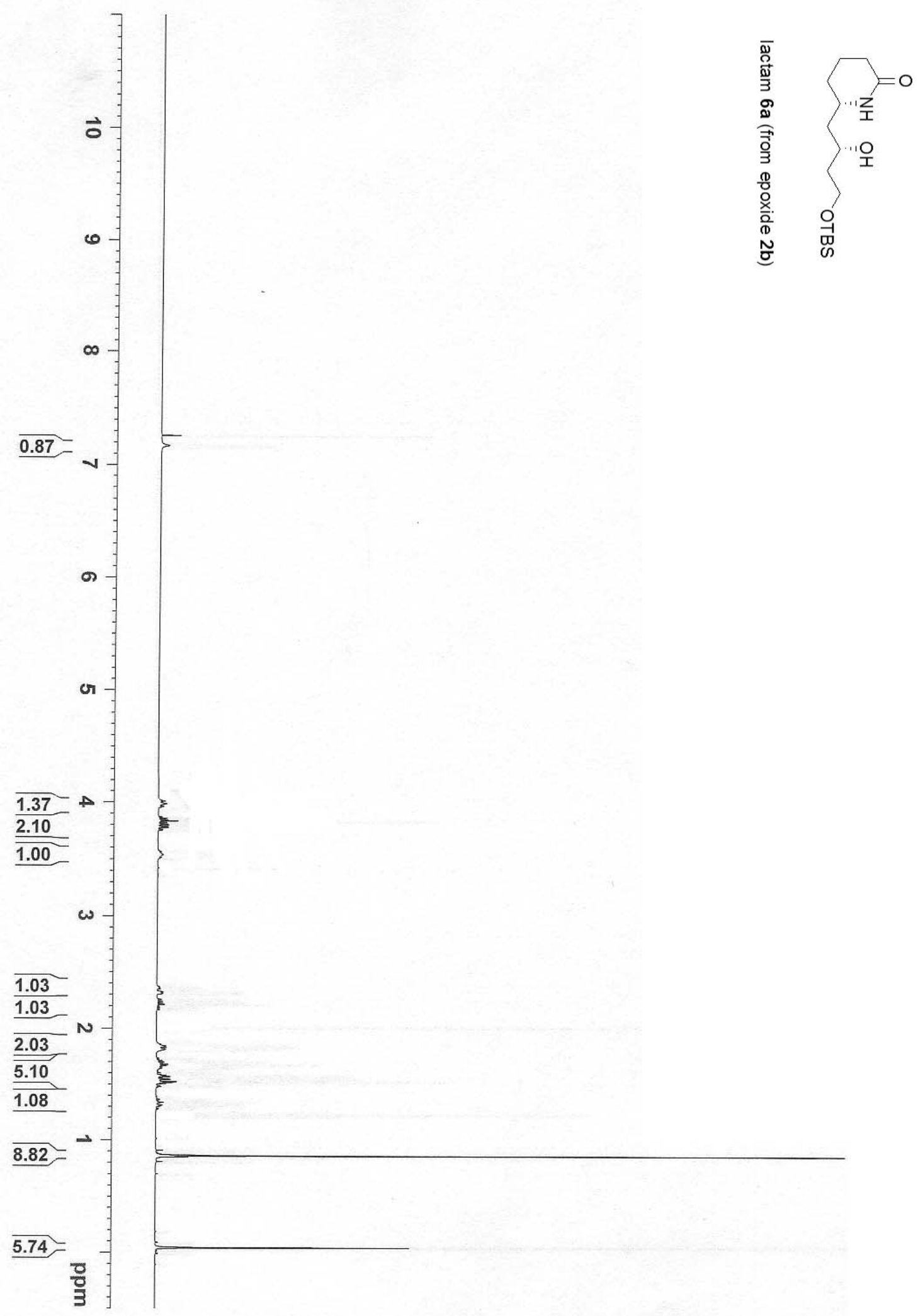




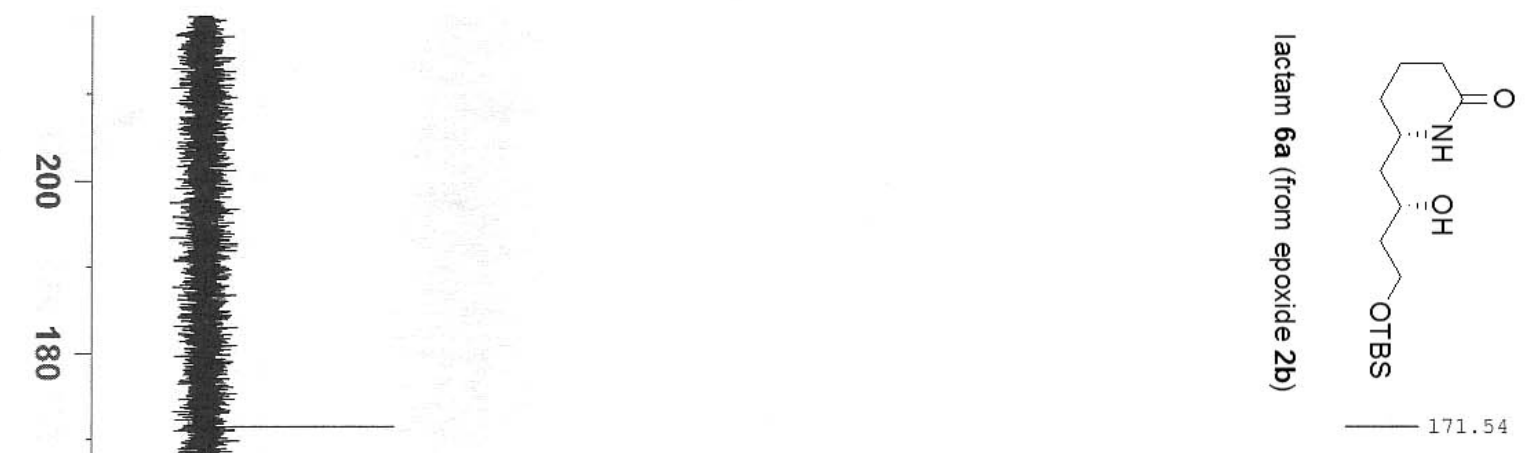

$\overrightarrow{8}$

$\vec{f}$

$\overrightarrow{\tilde{N}}$

$\vec{\circ}$

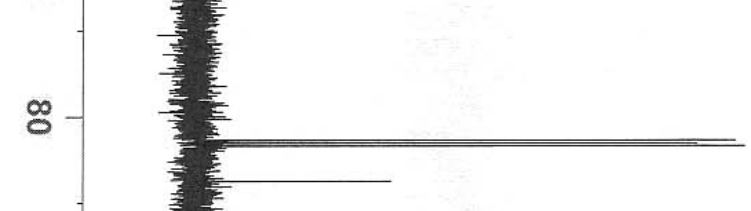

$\begin{array}{r}77.32 \\ -\quad 77.00 \\ \hline\end{array}$ 76.69
-76.57

8

음

응

o

总 


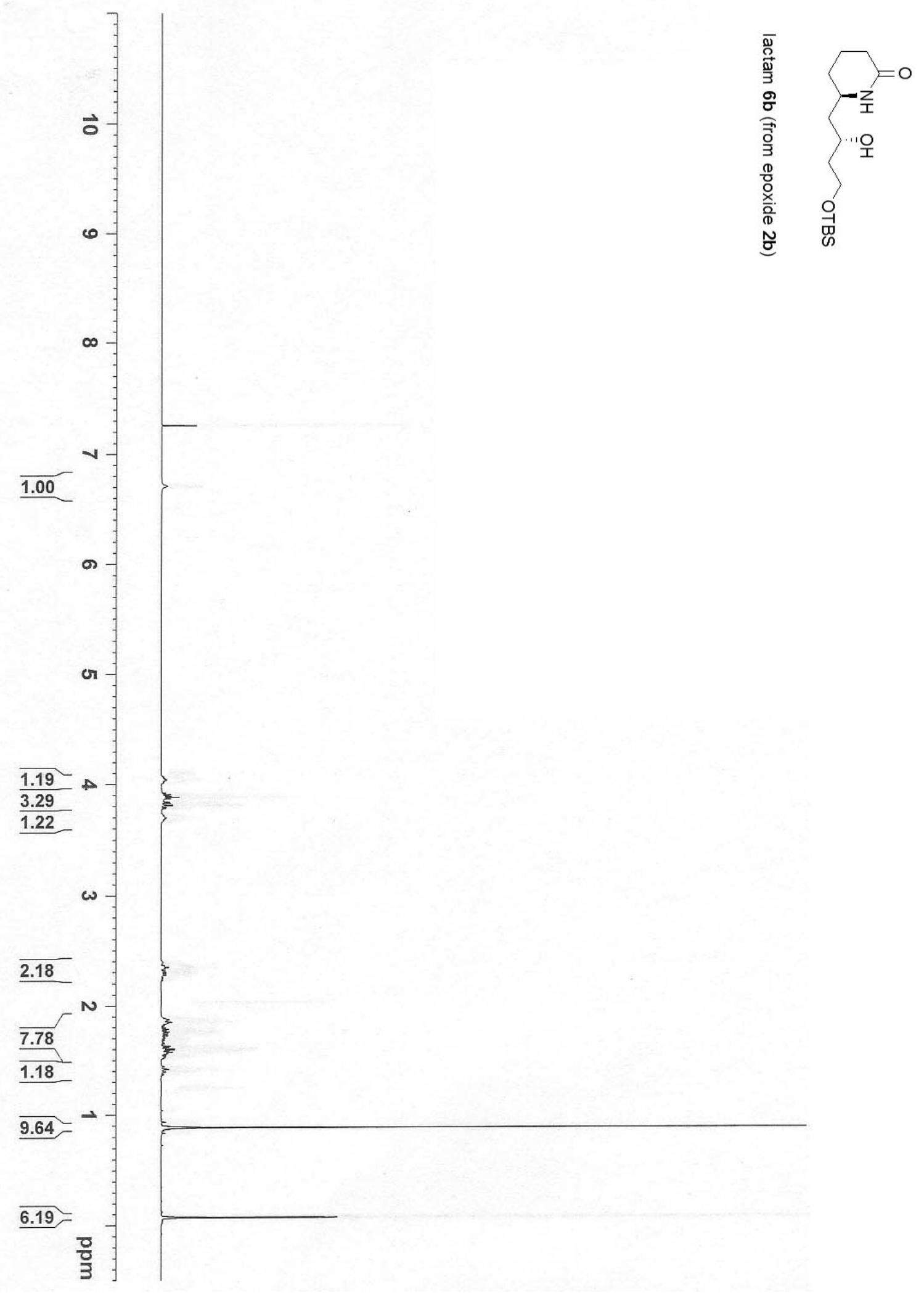



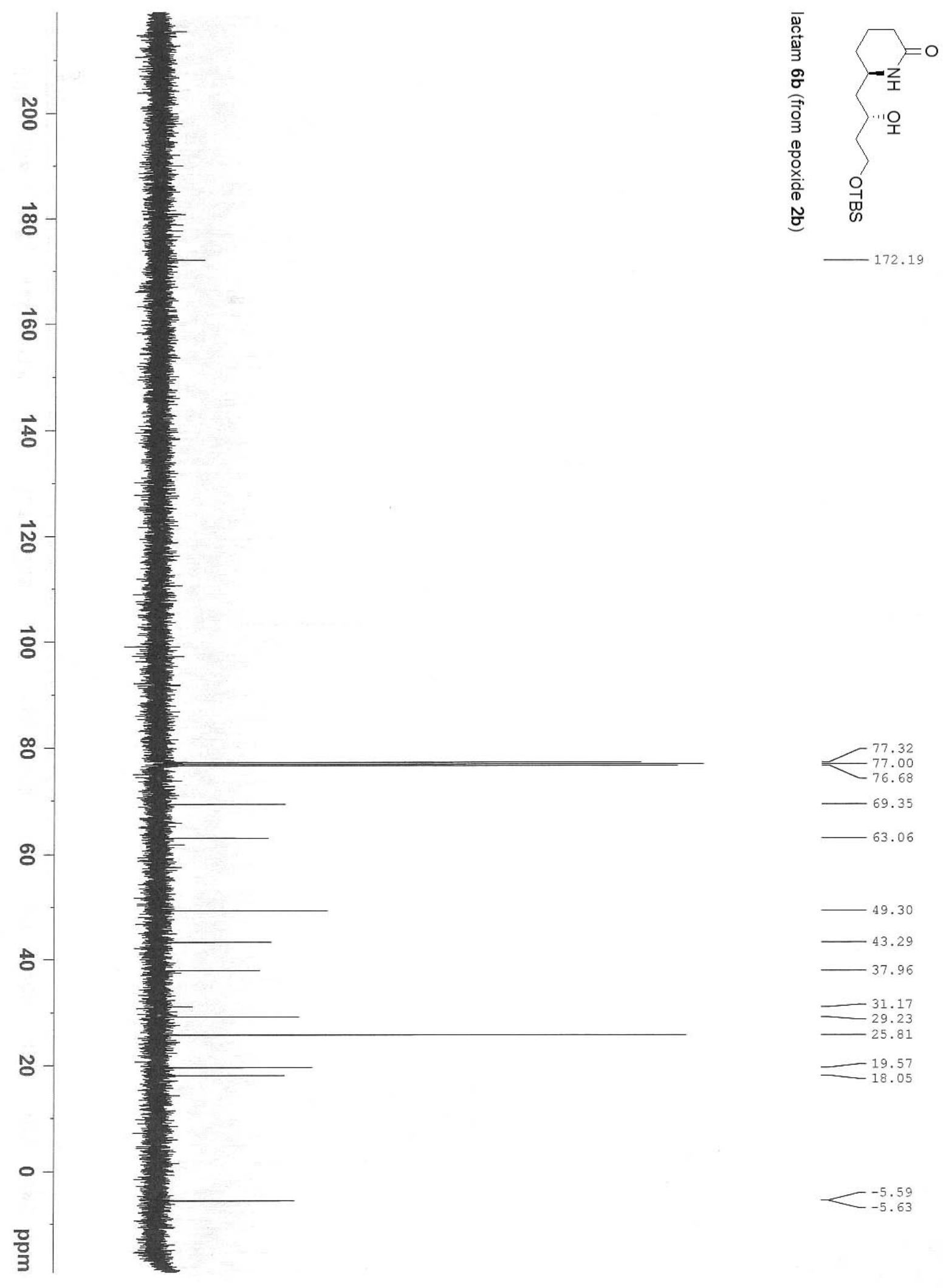


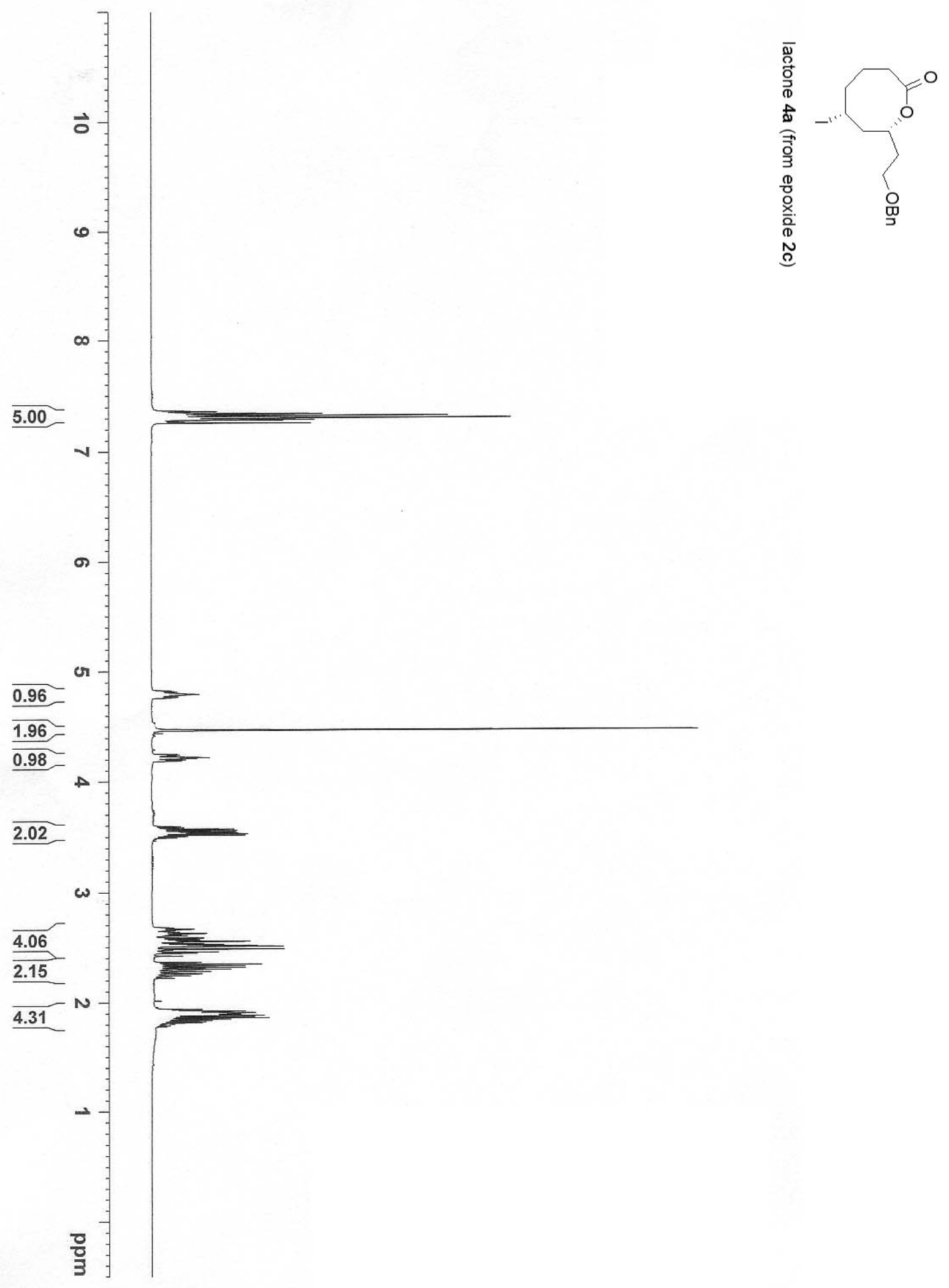



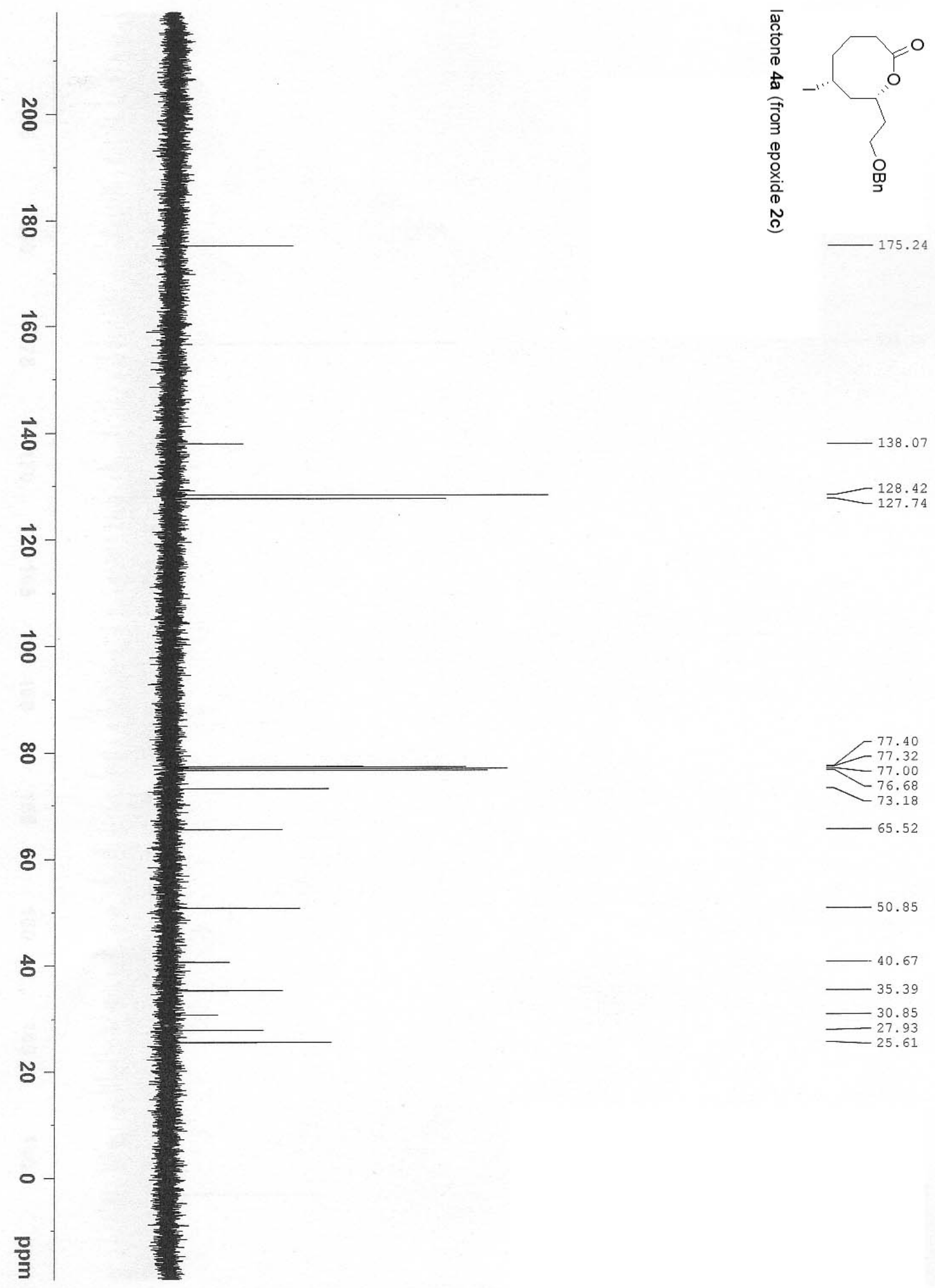

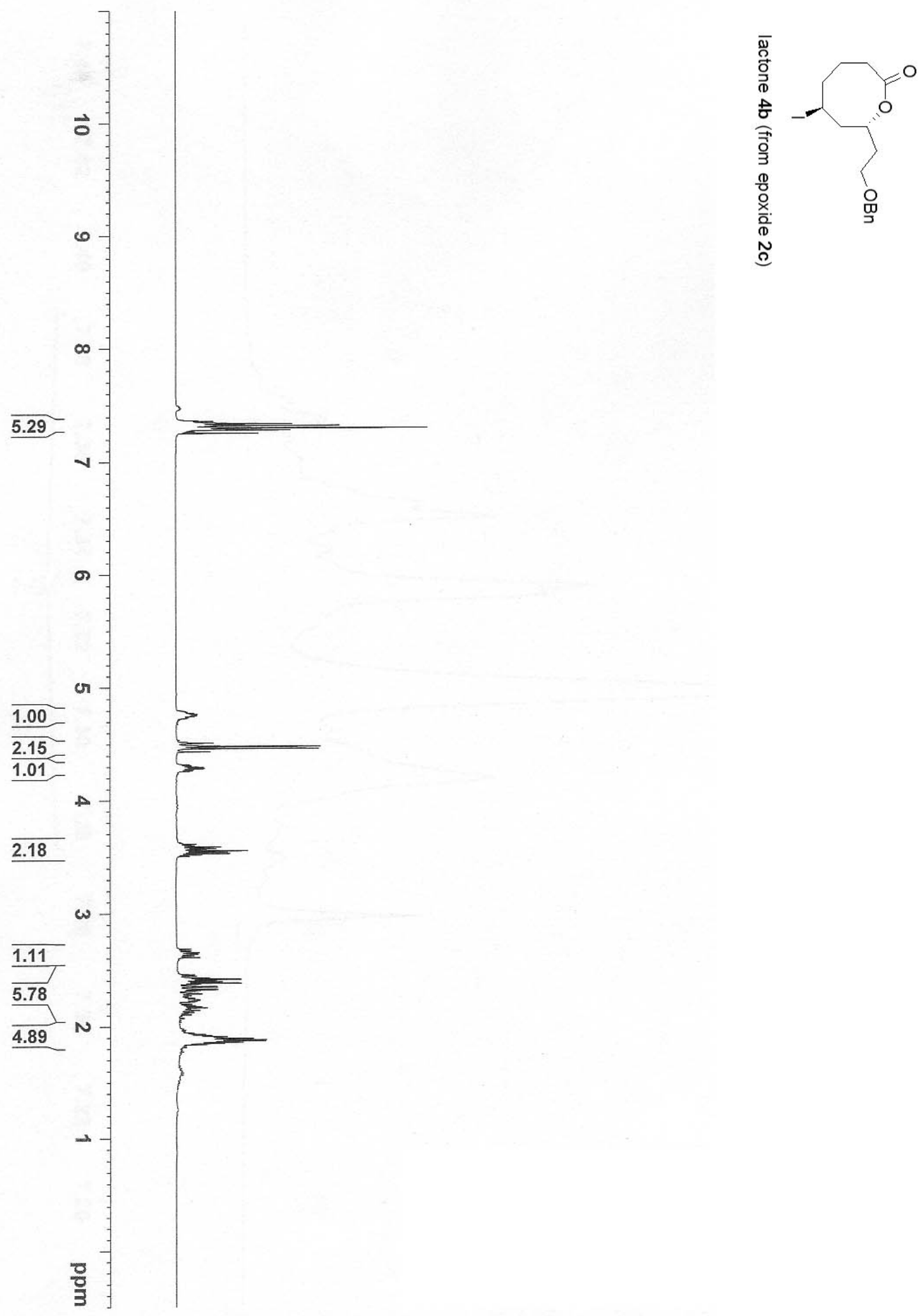

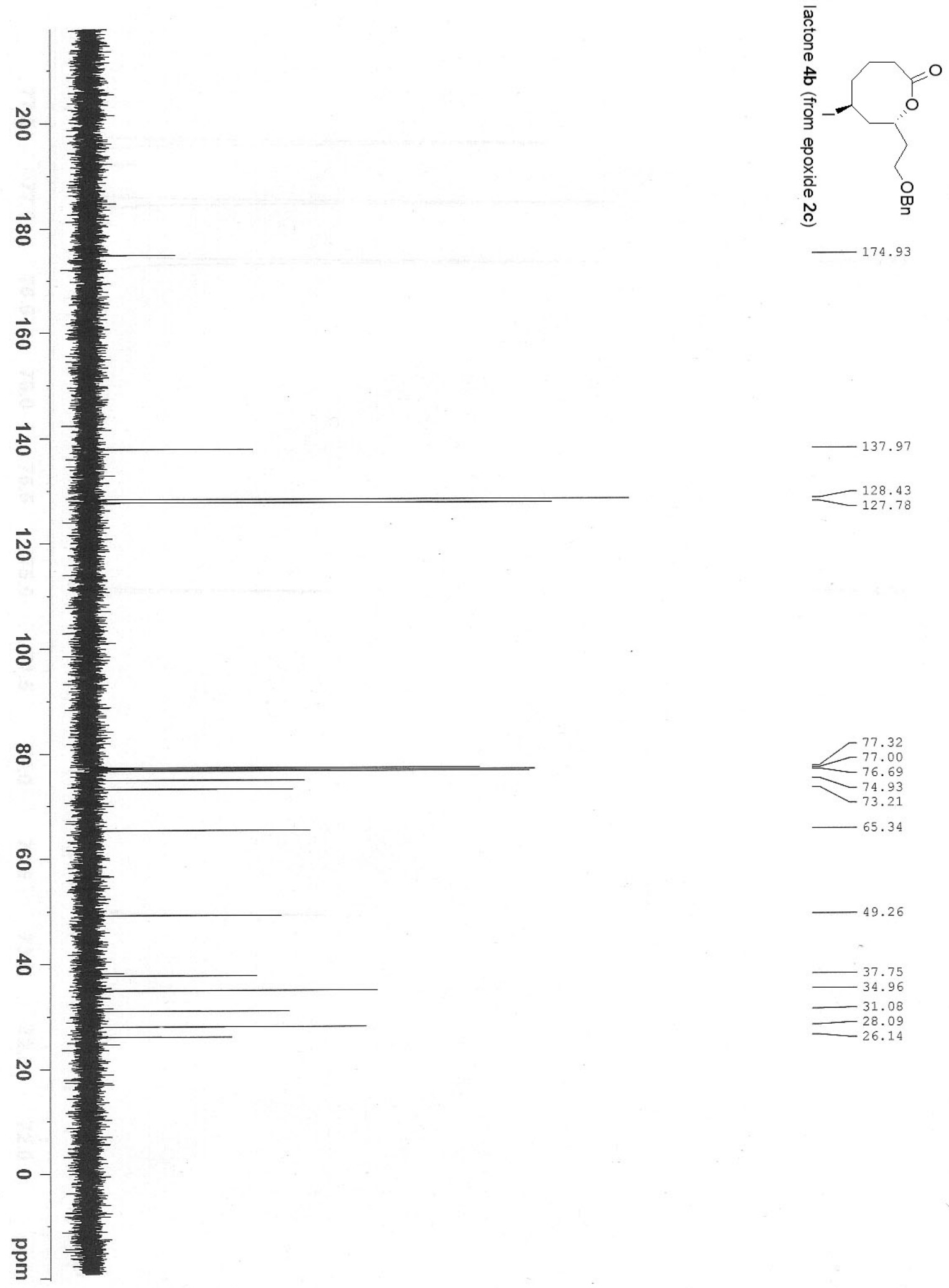

$-49.26$

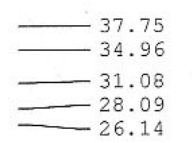



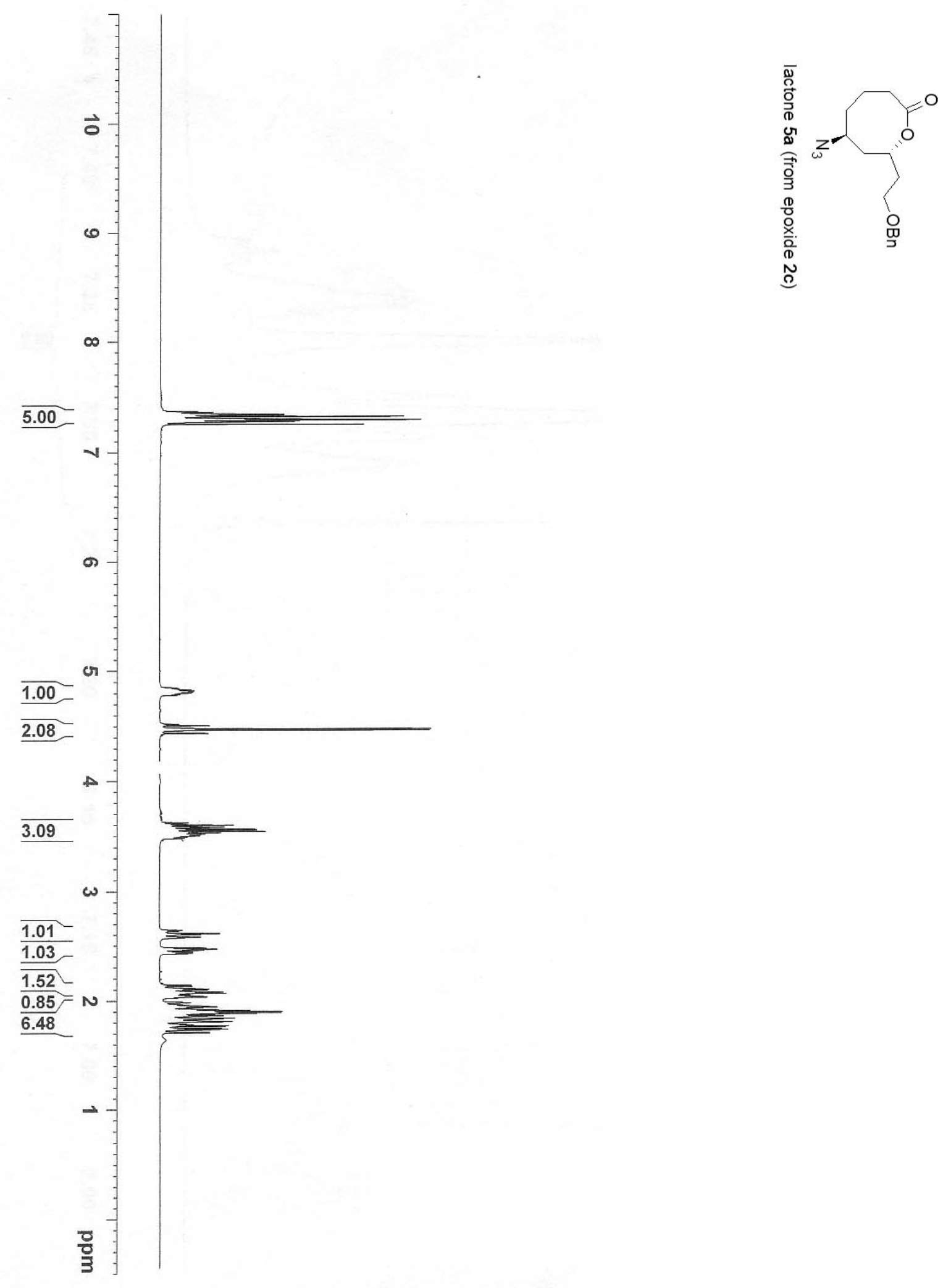

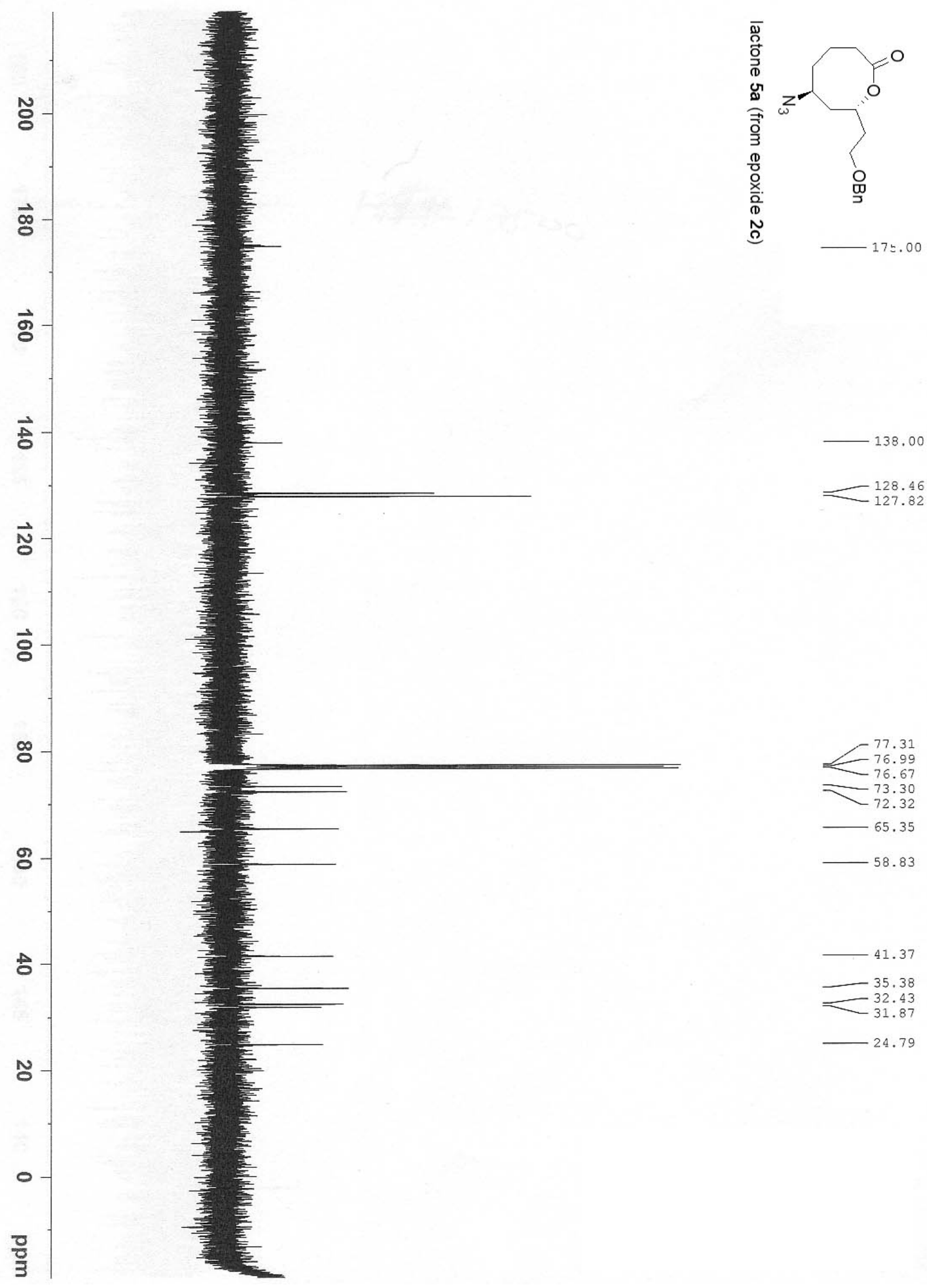

$-76.99$

$-76.67$ 73.30

72.32

65.35 $-58.83$ 


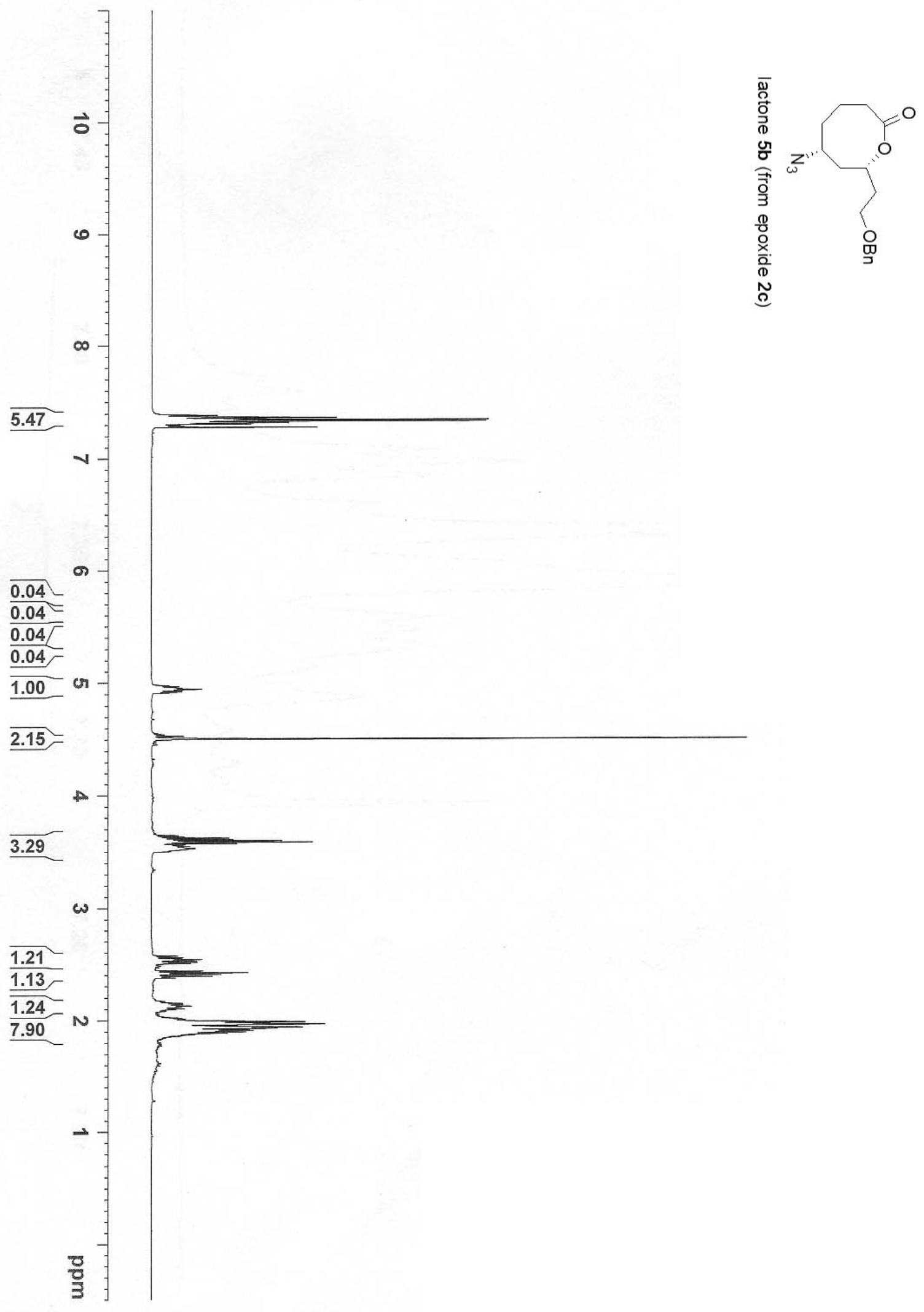



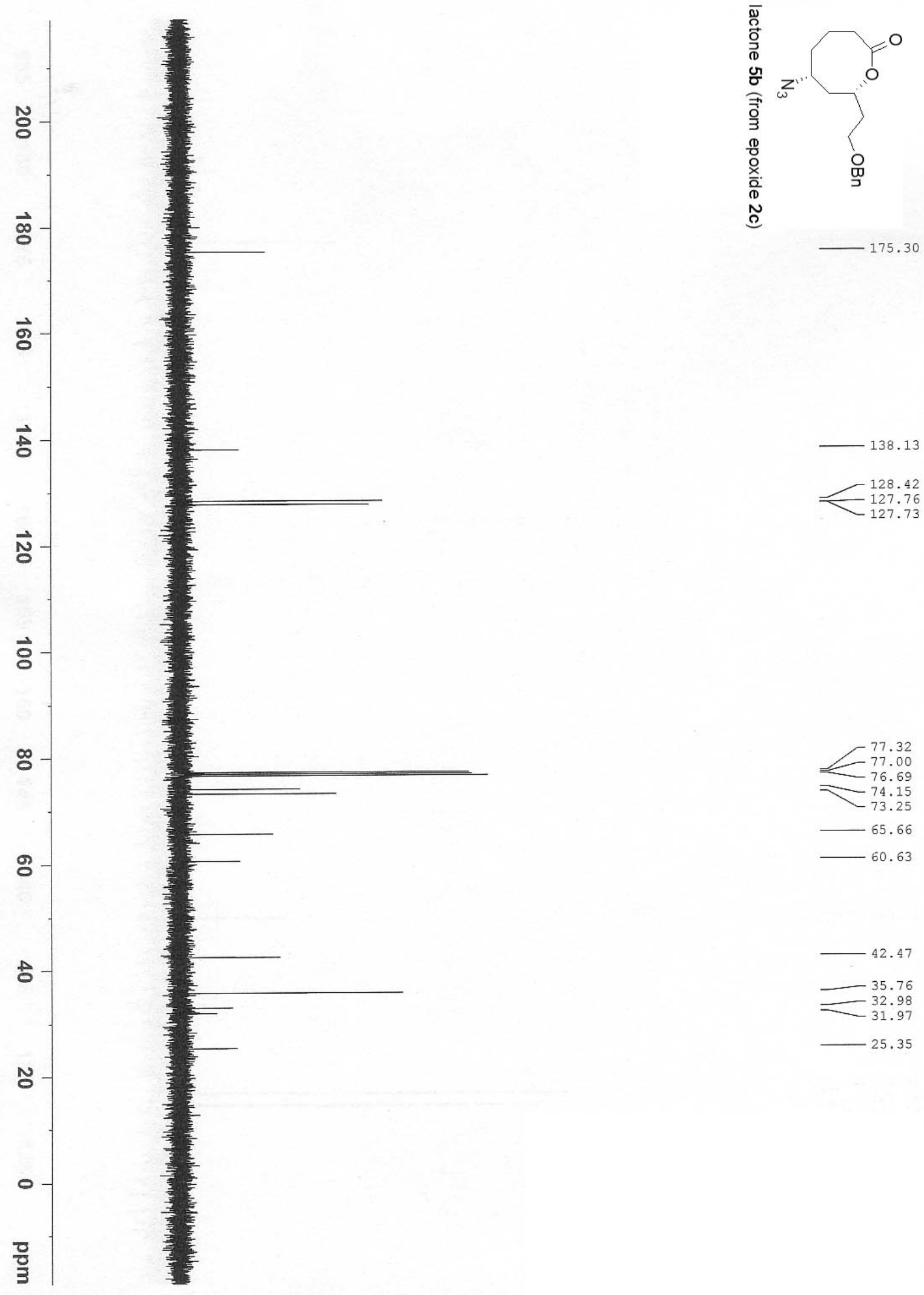

$\begin{array}{r}77.32 \\ -77.00 \\ \hline\end{array}$

$\begin{array}{r}77.00 \\ -76.69 \\ \hline\end{array}$

$-74.15$

$-65.66$

$-60.63$

$-42.47$

$\begin{array}{r}-35.76 \\ -\quad 32.98 \\ \hline\end{array}$

31.97 $-25.35$ 


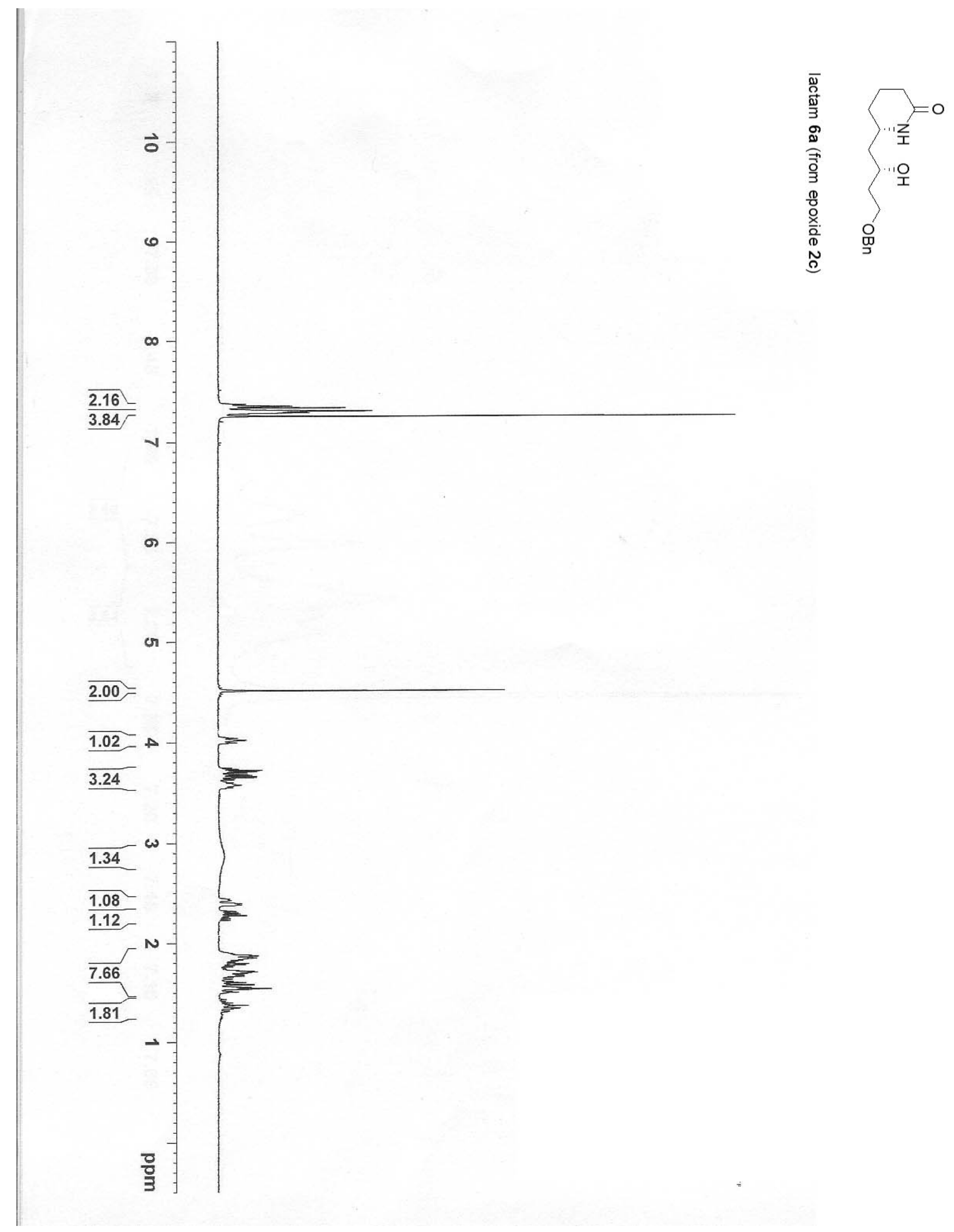




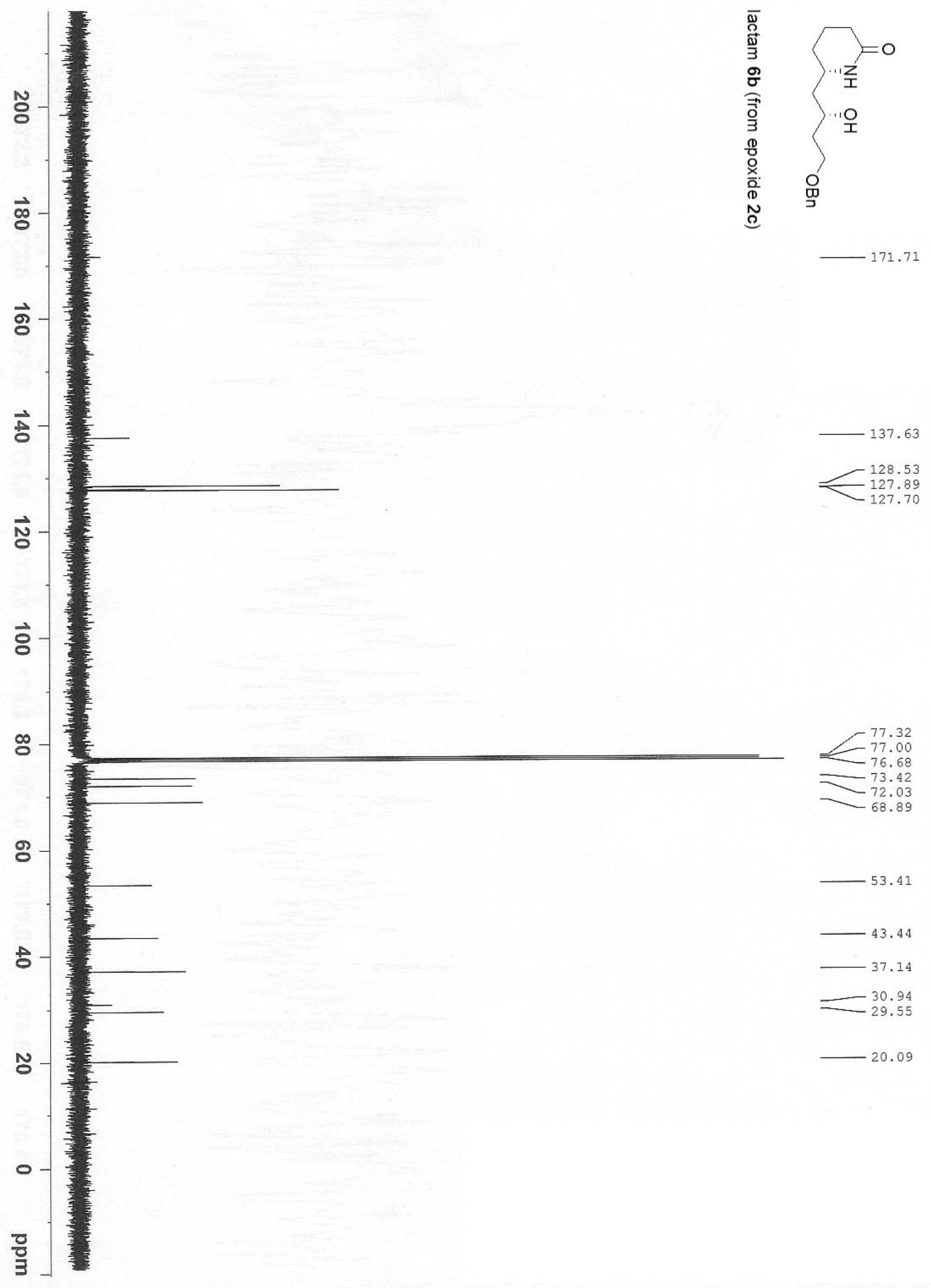




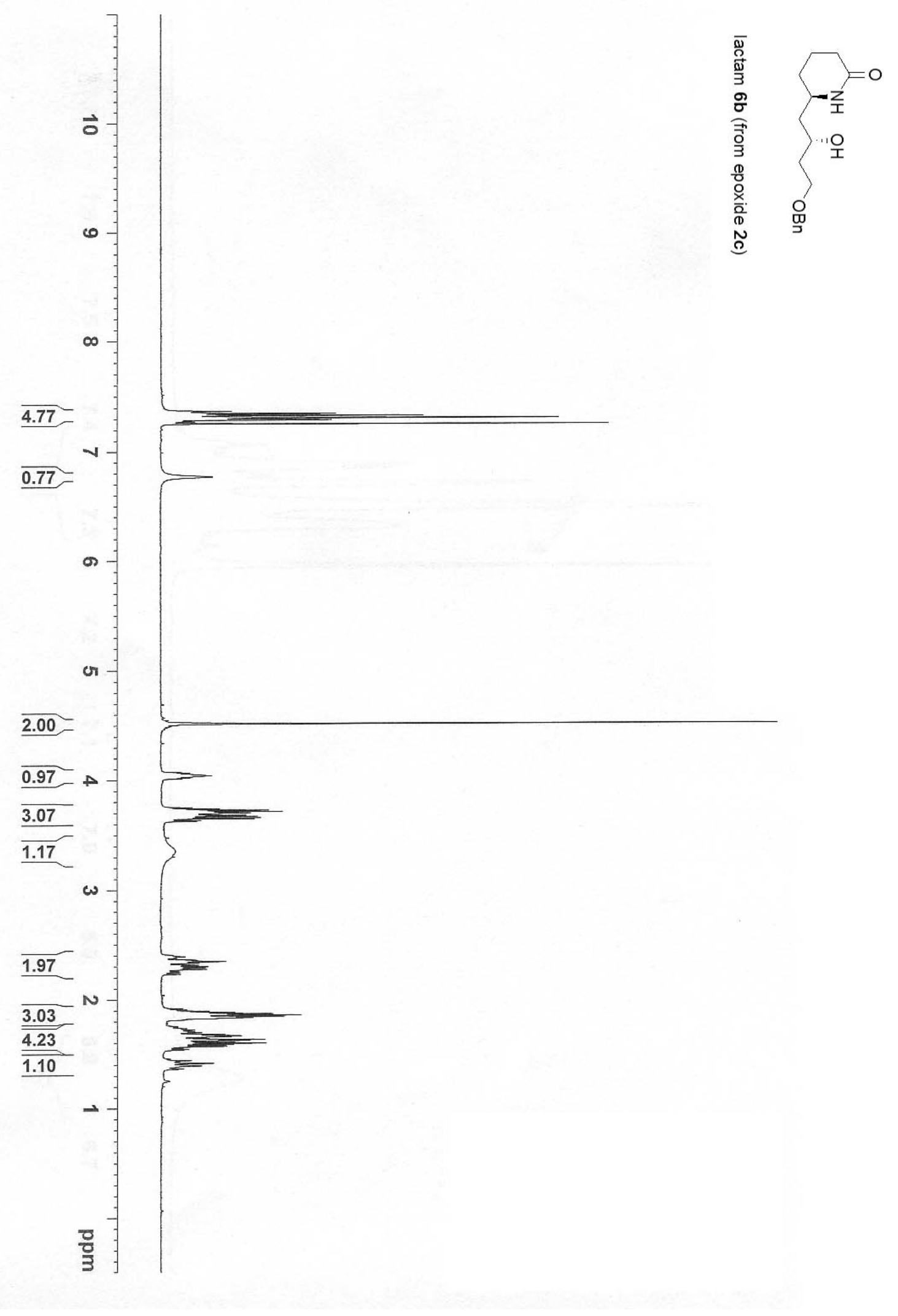




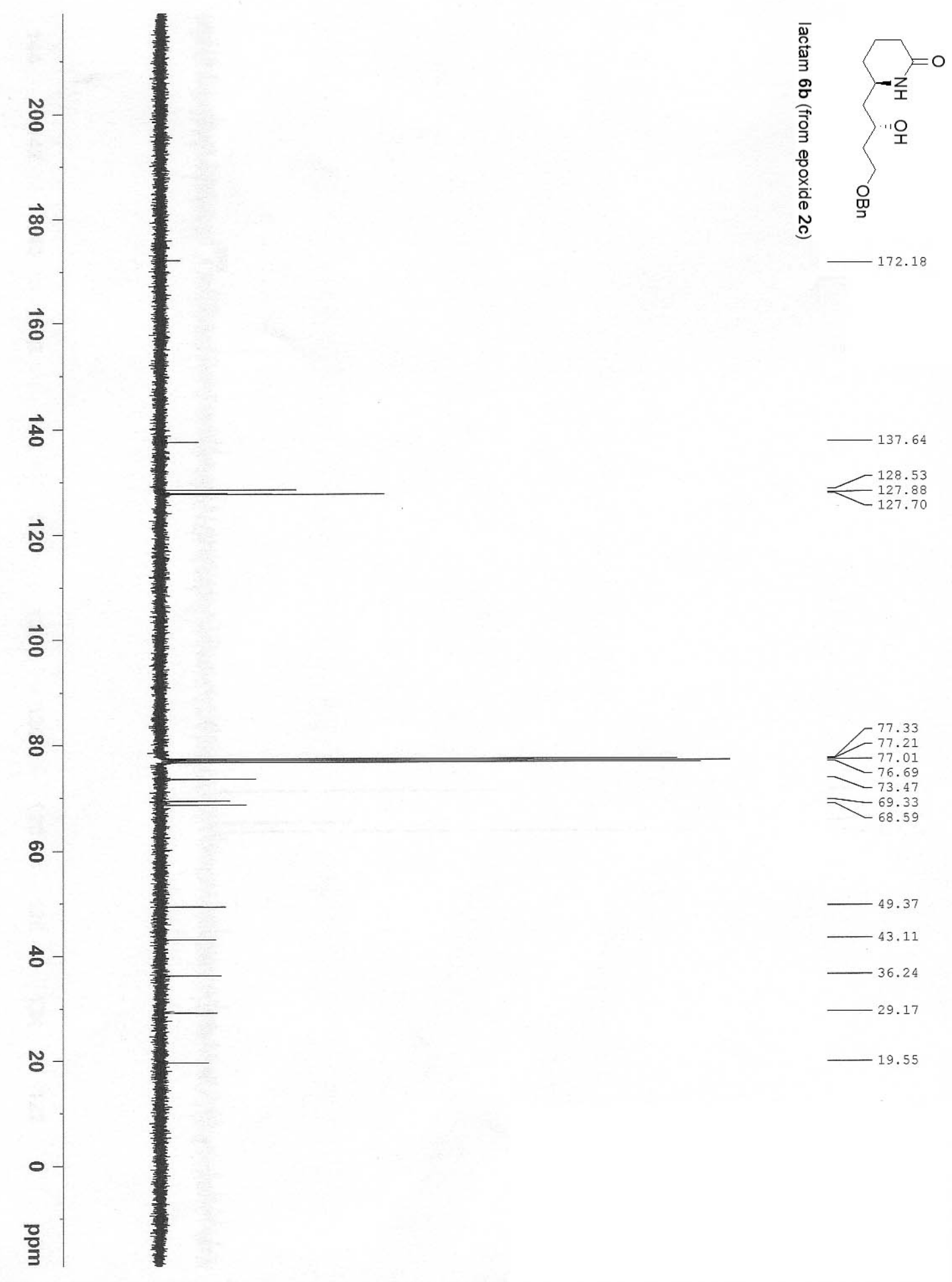




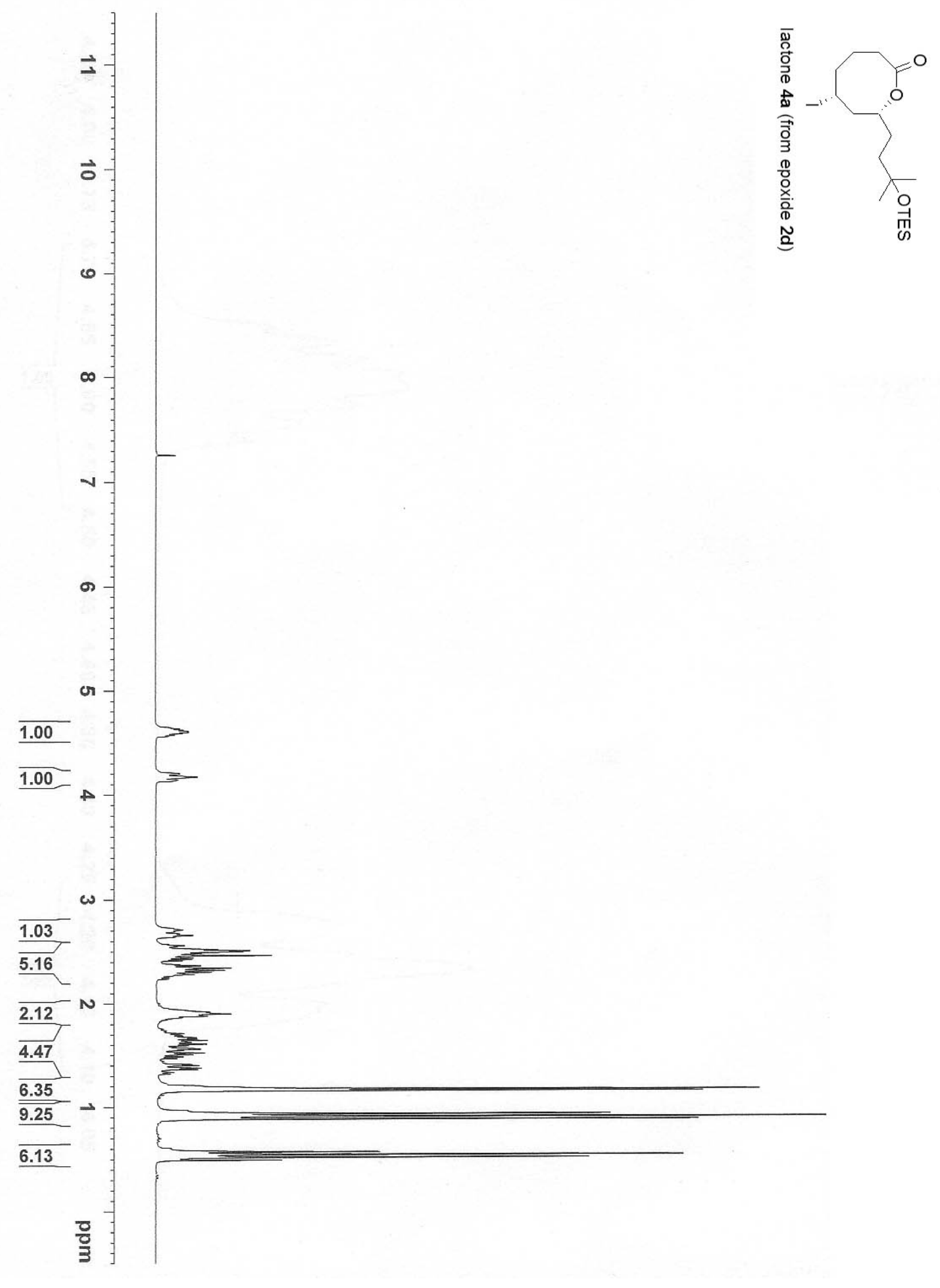



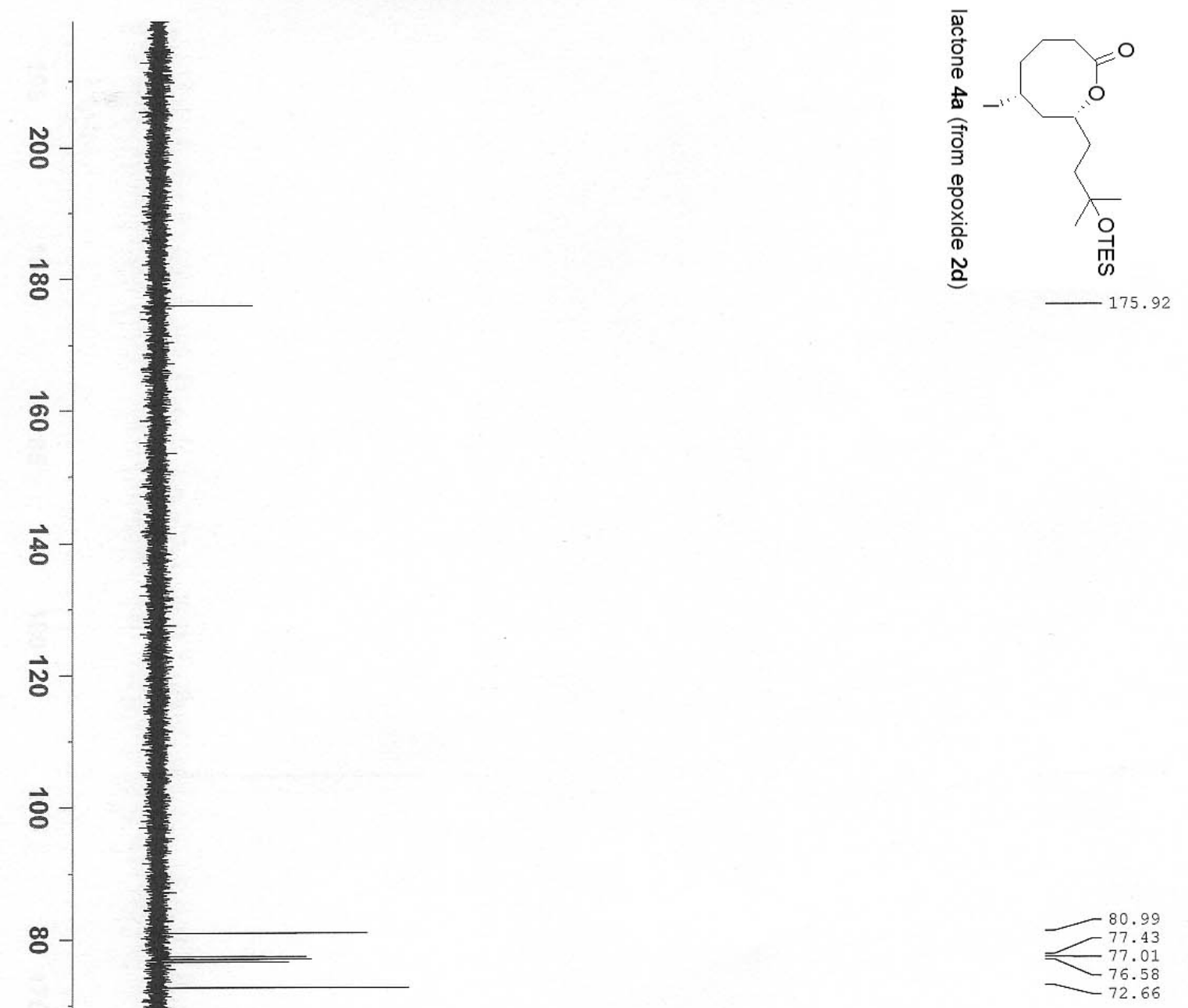

$-51.59$

ㅇ

$\overrightarrow{8}$

ஃ

$\overrightarrow{8}$

$\vec{\Delta}$

$\vec{N}$

8

ก

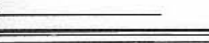

ธ

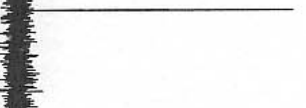

-41.59
$-\quad 40.48$

$-31.86$

31.86
-30.10

-29.94
-29.48

-28.59
-25.99

응 


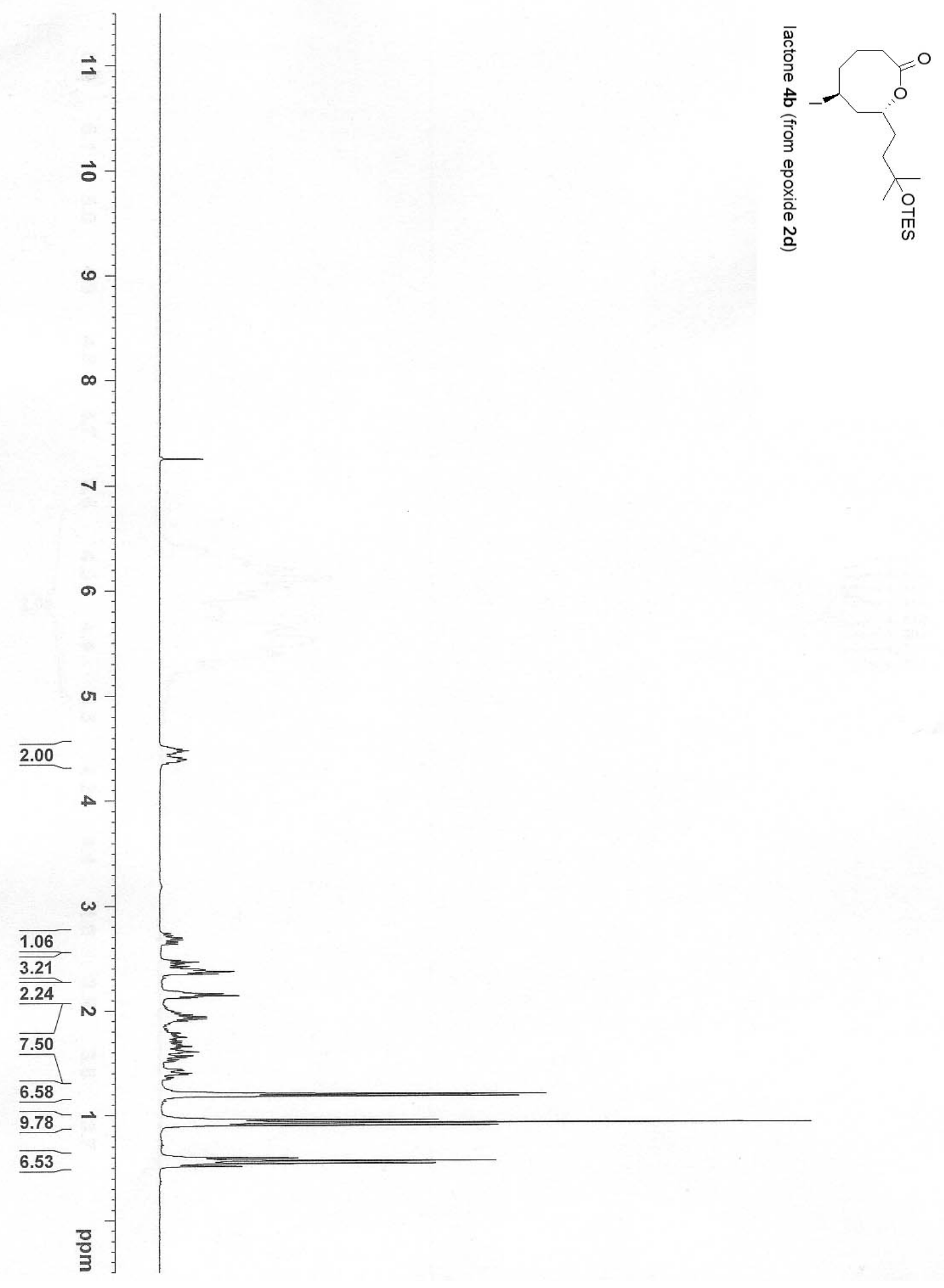




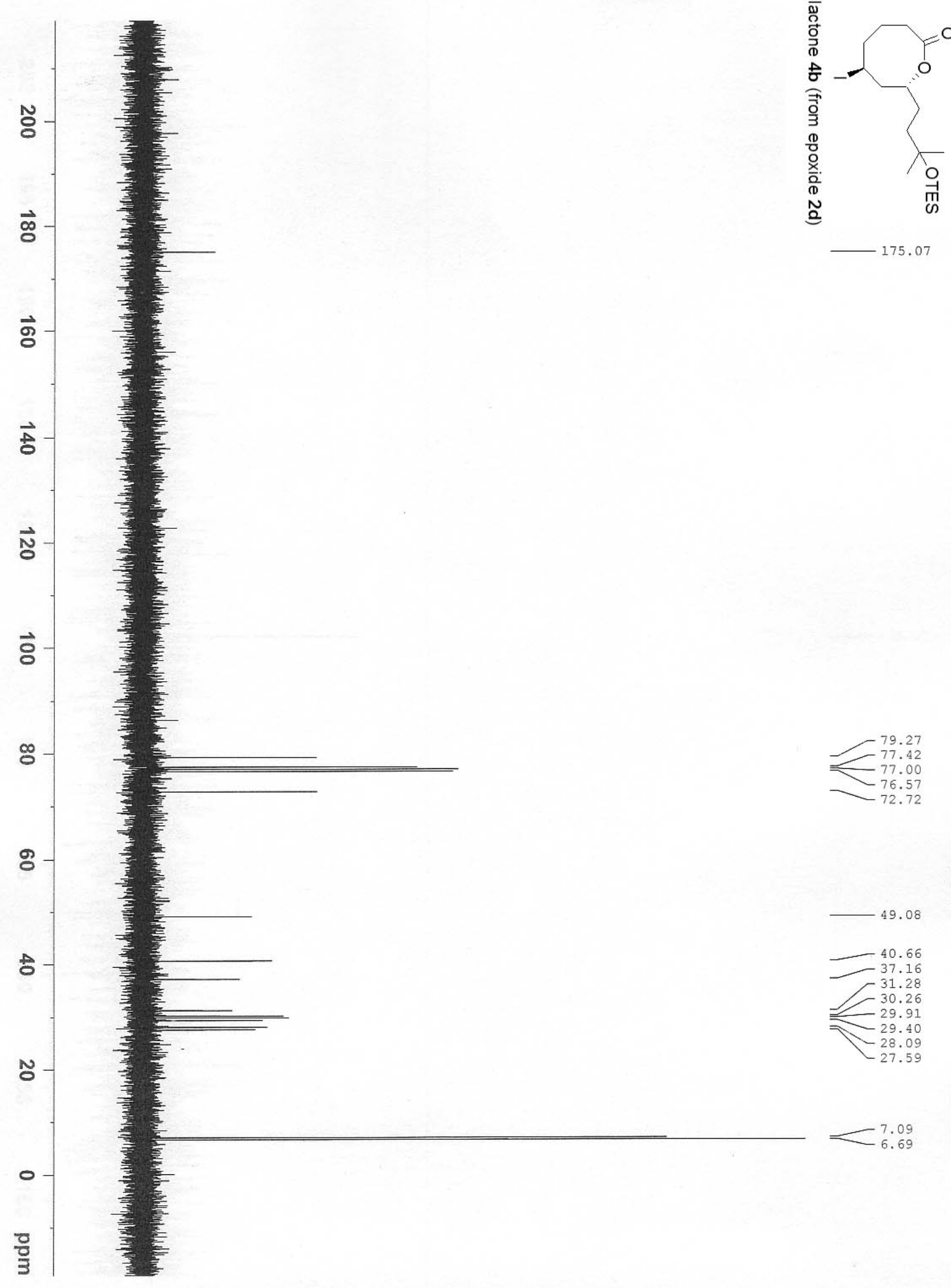




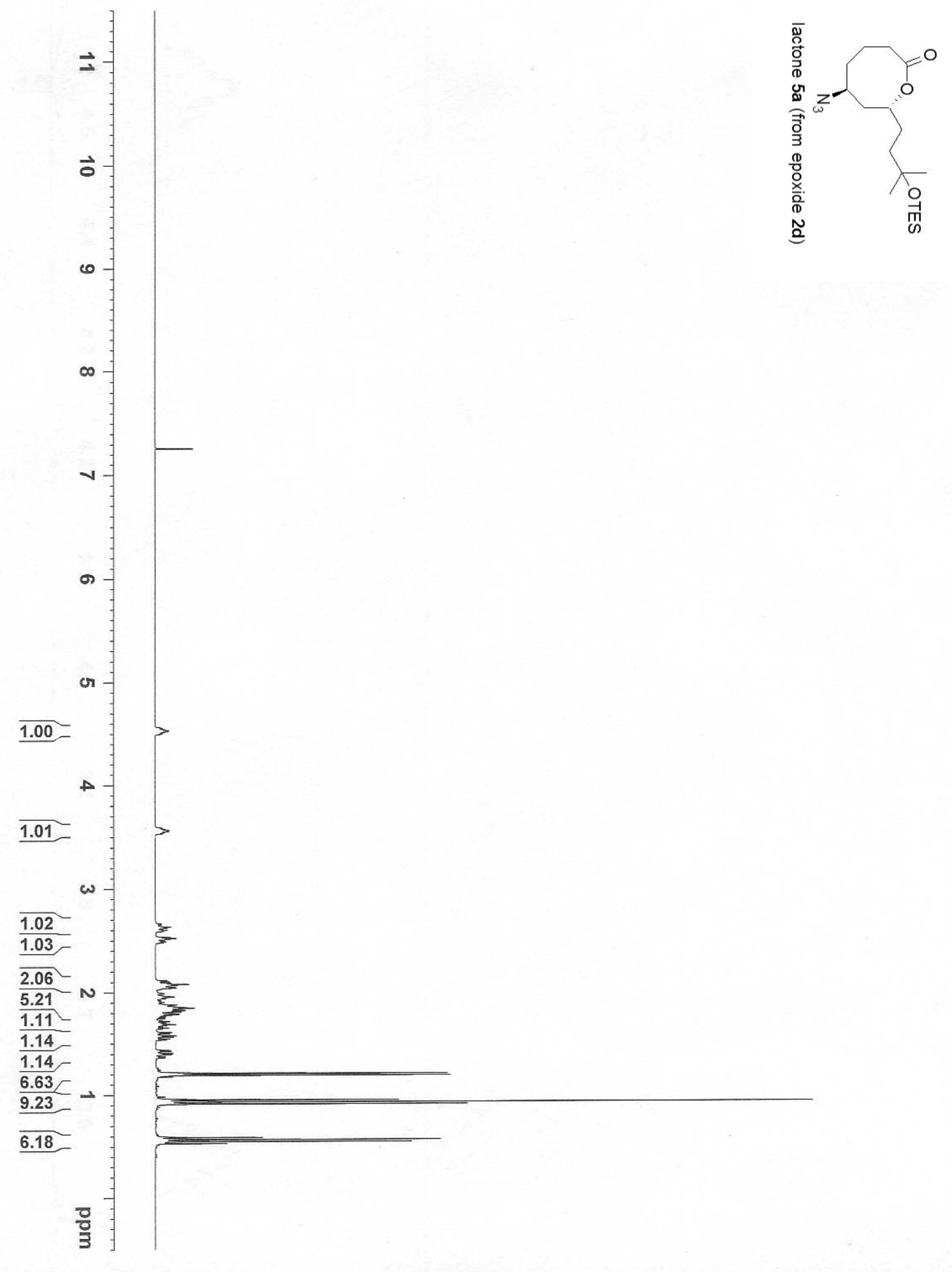




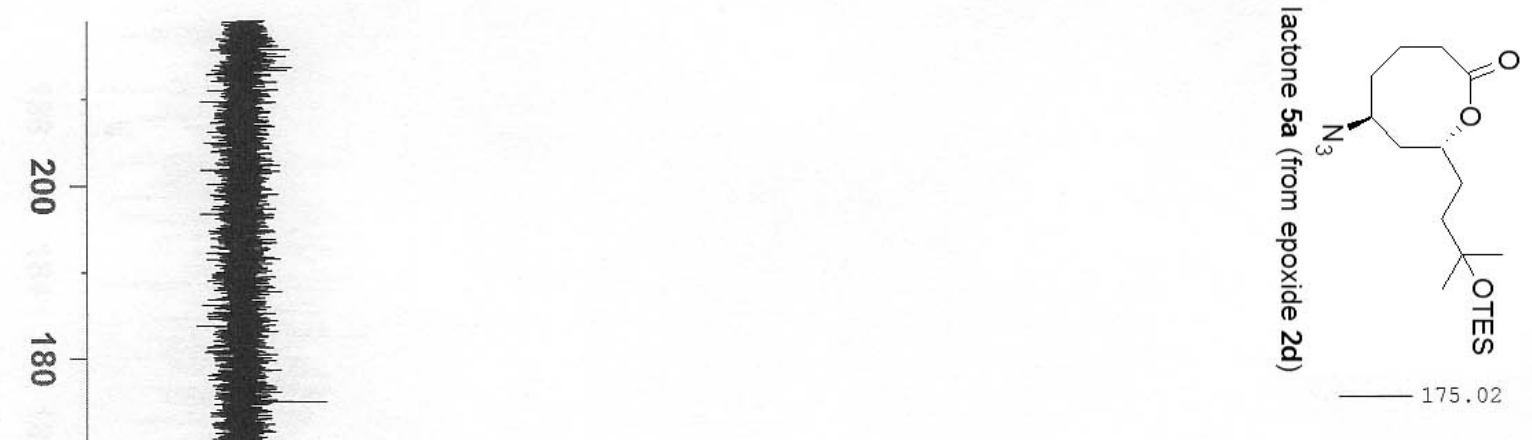

के

용

$\overrightarrow{\text { o }}$

$\vec{N}$

$\vec{\circ}$

$\stackrel{\infty}{\circ}$

o

g

8-

bे

o -

ธ

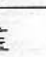

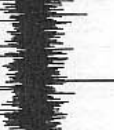

를

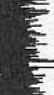

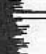
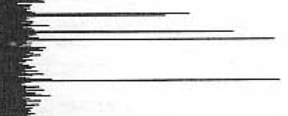

77.32
77.00

$\begin{array}{r}76.69 \\ \hline 76.76\end{array}$

-76.60
-72.76

$-58.73$

$=41.52$ 32.13

-30.29
-30.25

$\checkmark 30.25$

-29.38
-24.55
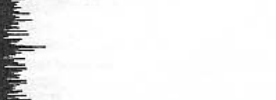

o

蛋

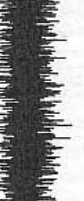

-7.08
+ 


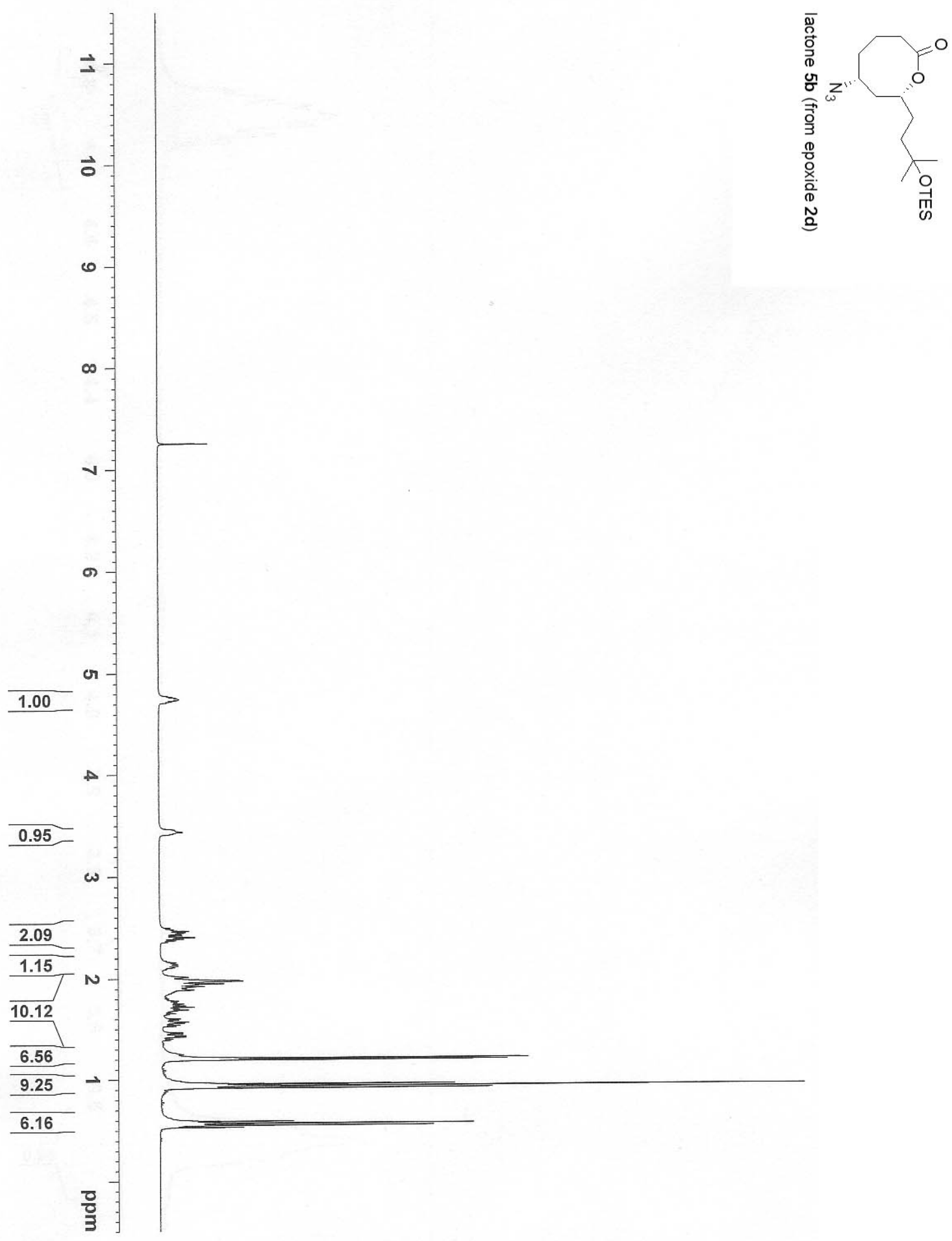



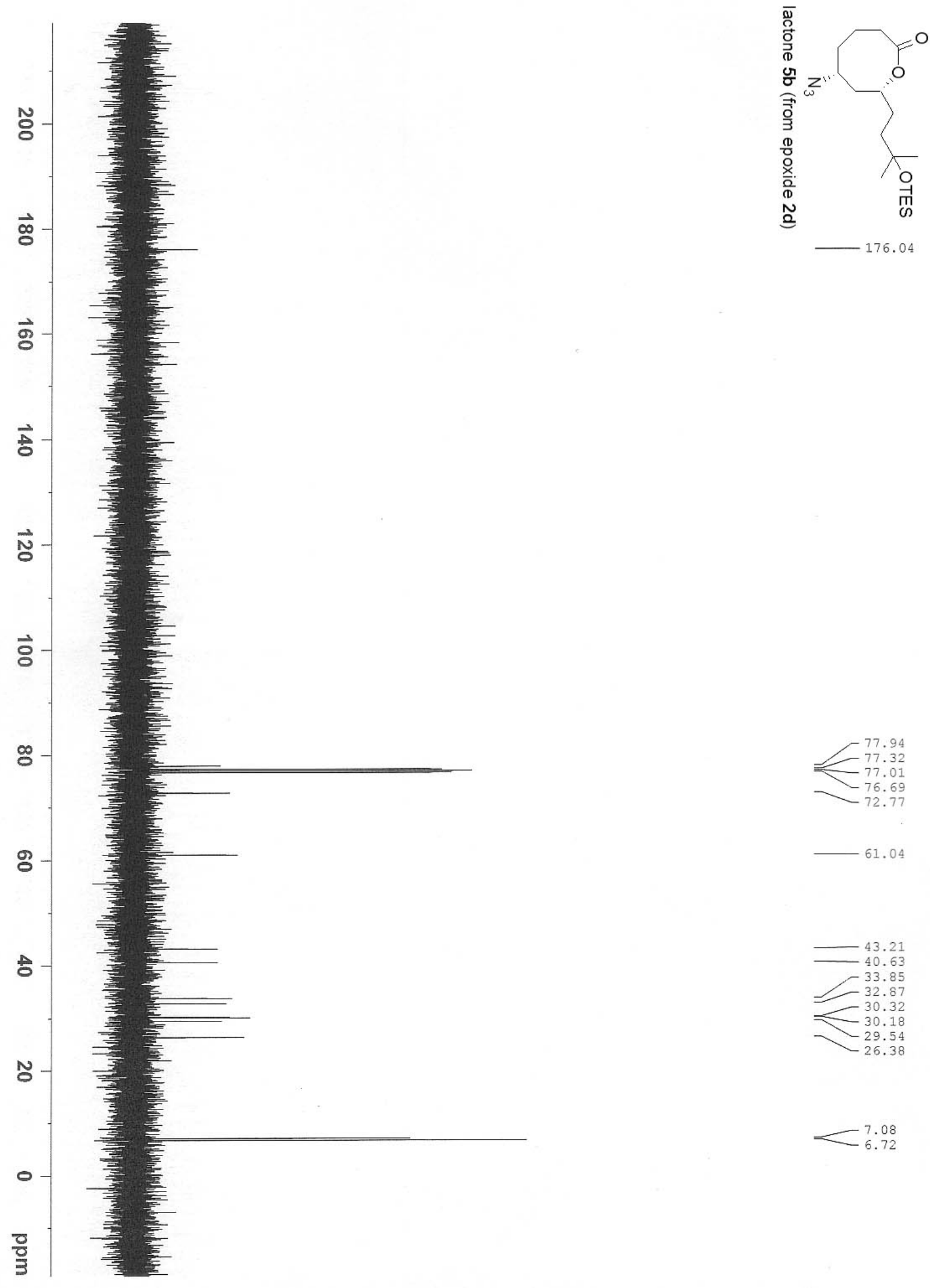


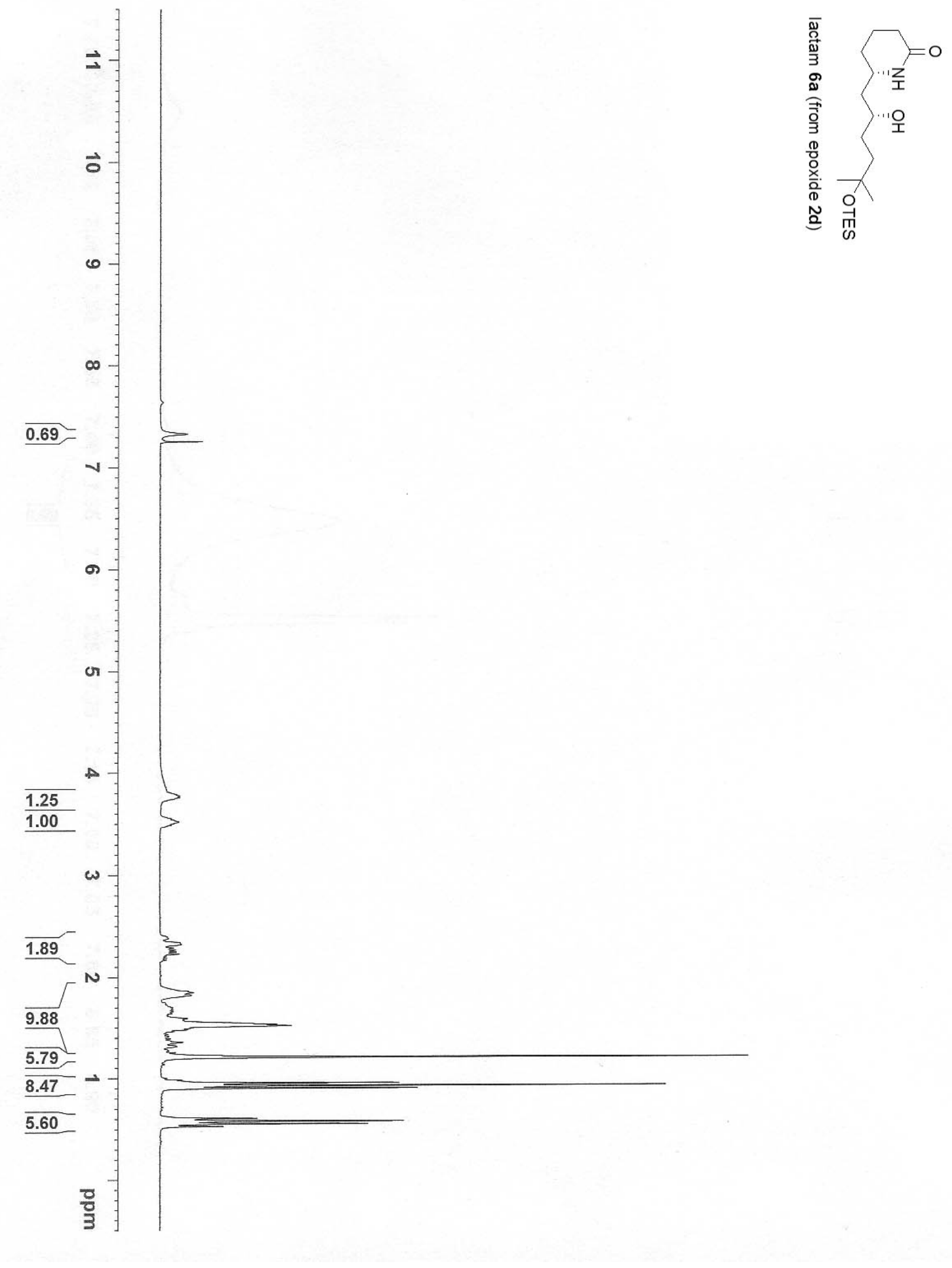




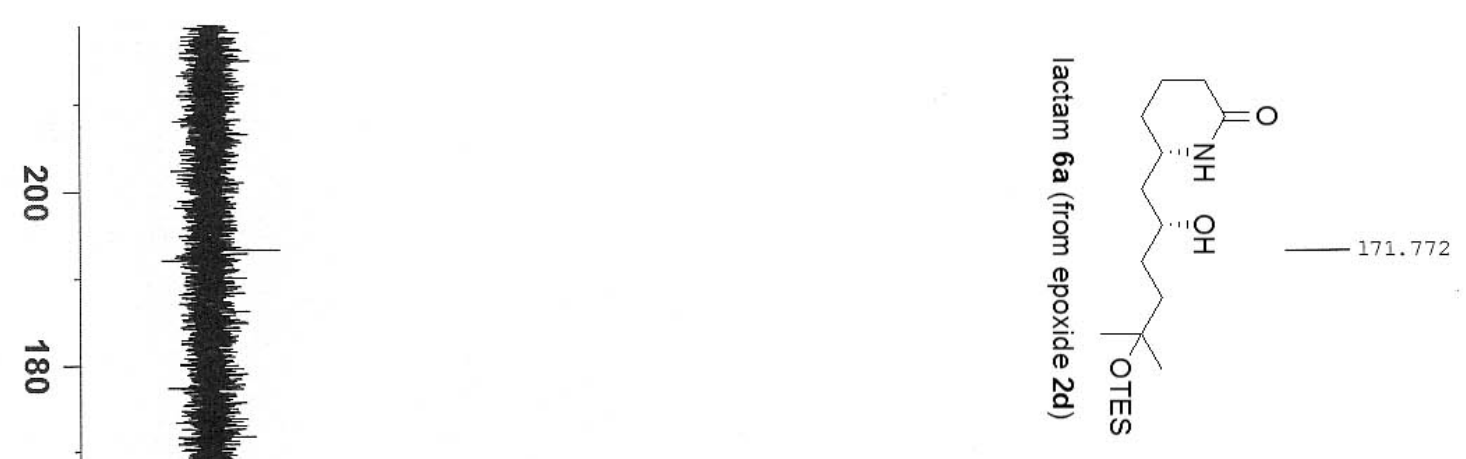

$-77.321$

$=77.002$

$-73.560$

$-72.484$

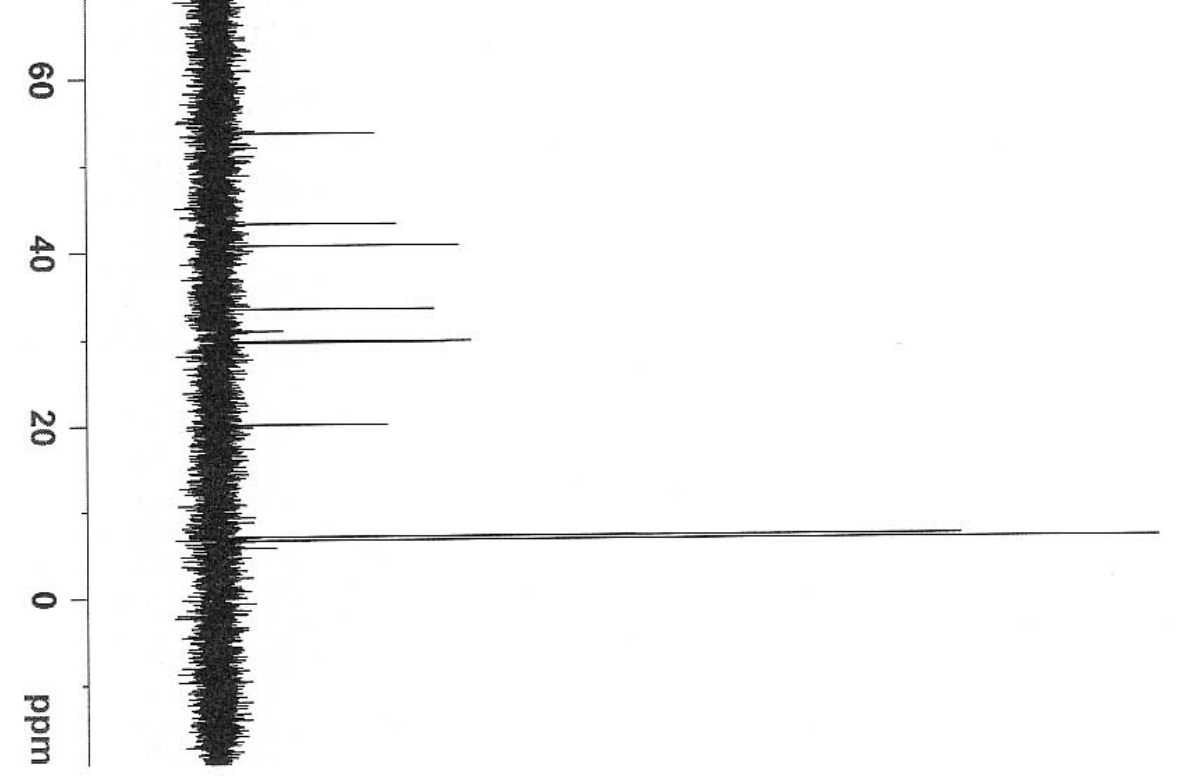

$-53.712$

$\begin{array}{r}43.287 \\ \hline \\ \hline\end{array} 0.762$

$-33.474$

$-30.945$

29.801

29.768
-29.649

20.107

-7.012
$=6.604$ 


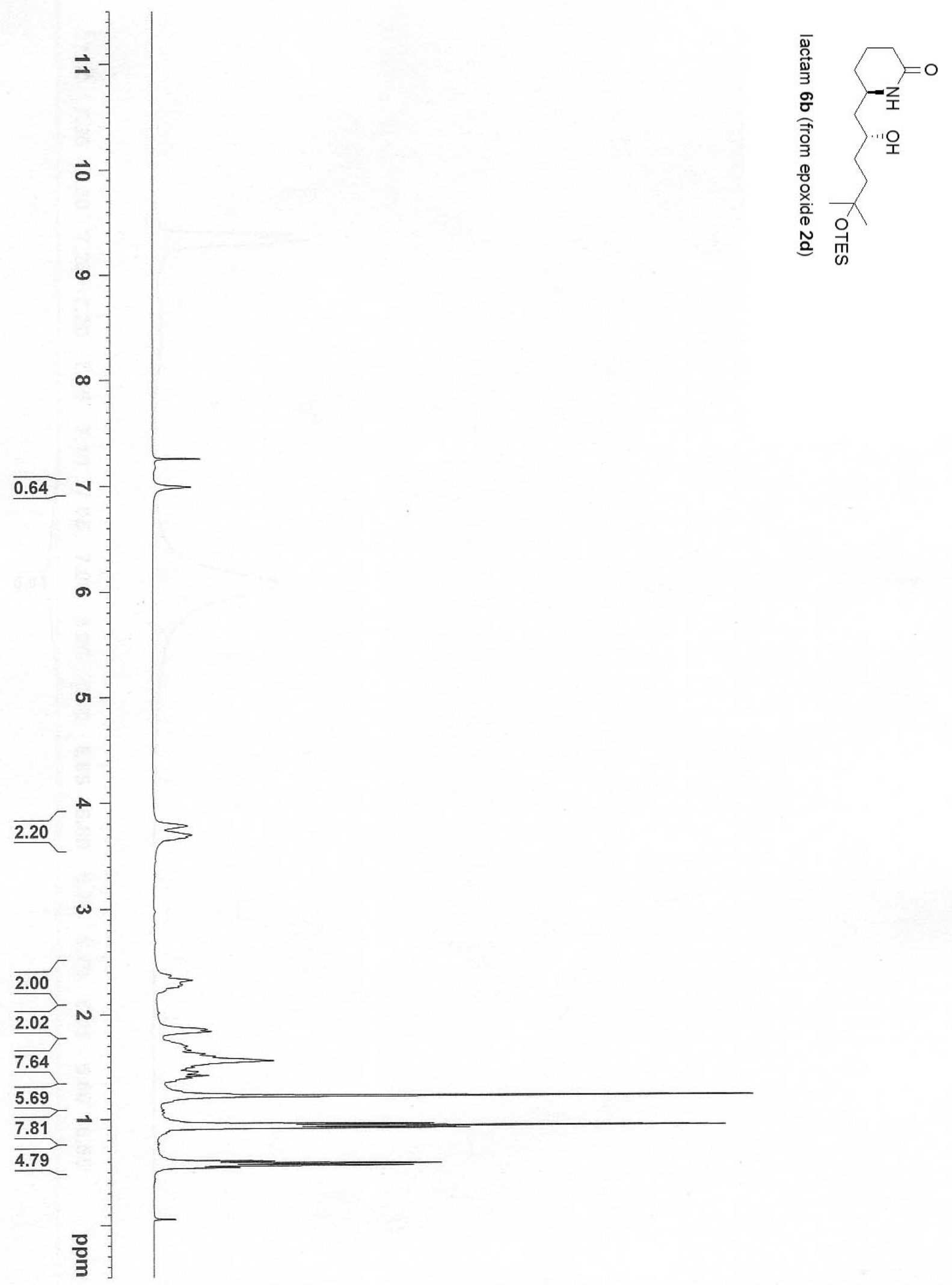




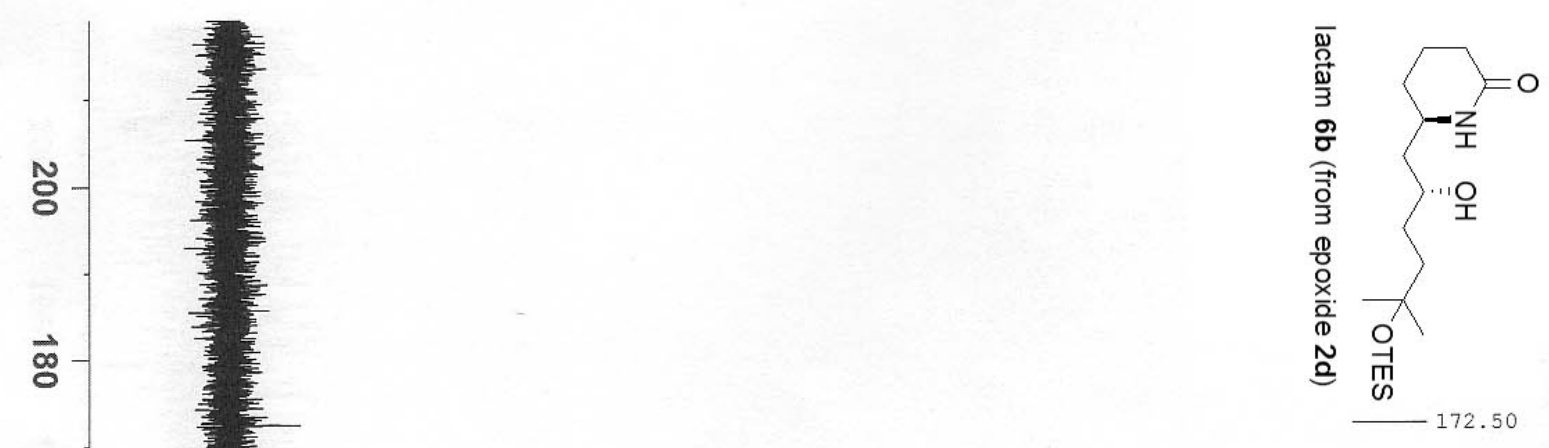

홍

$\overrightarrow{\text { o }}$

$\overrightarrow{\mathrm{N}}$

हे

ஃ

\&

o

ก

-1

응

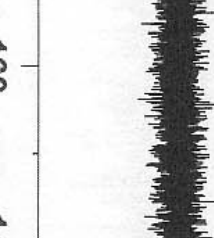

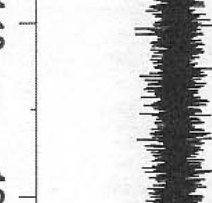

- 1 竞高

$\overrightarrow{-}$

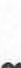

8

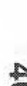

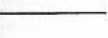

$-49.32$

$-43.11$

41.39

$-32.16$

$-31.20$

- 29.82

$\longrightarrow 29.15$

$-19.51$ 

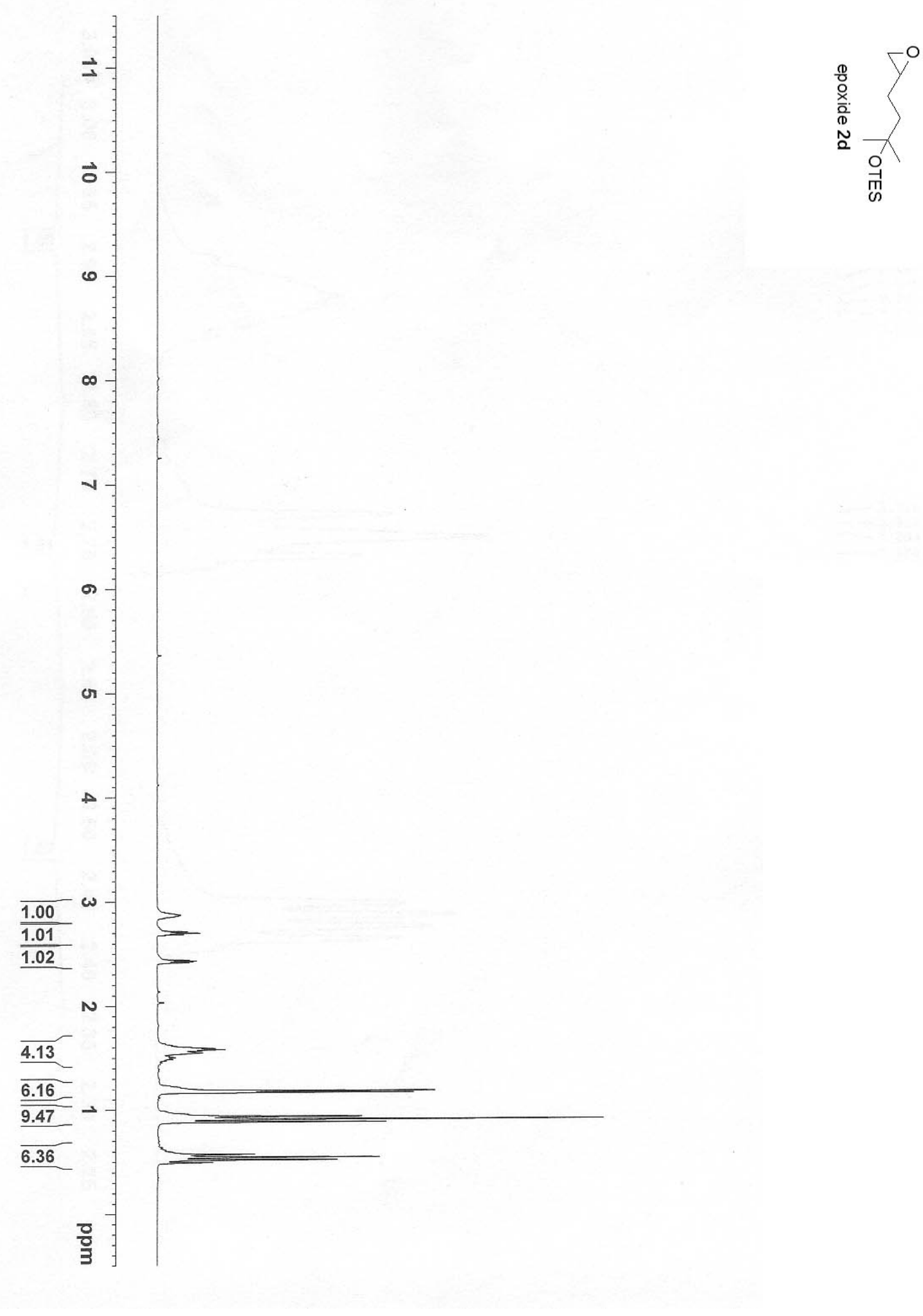

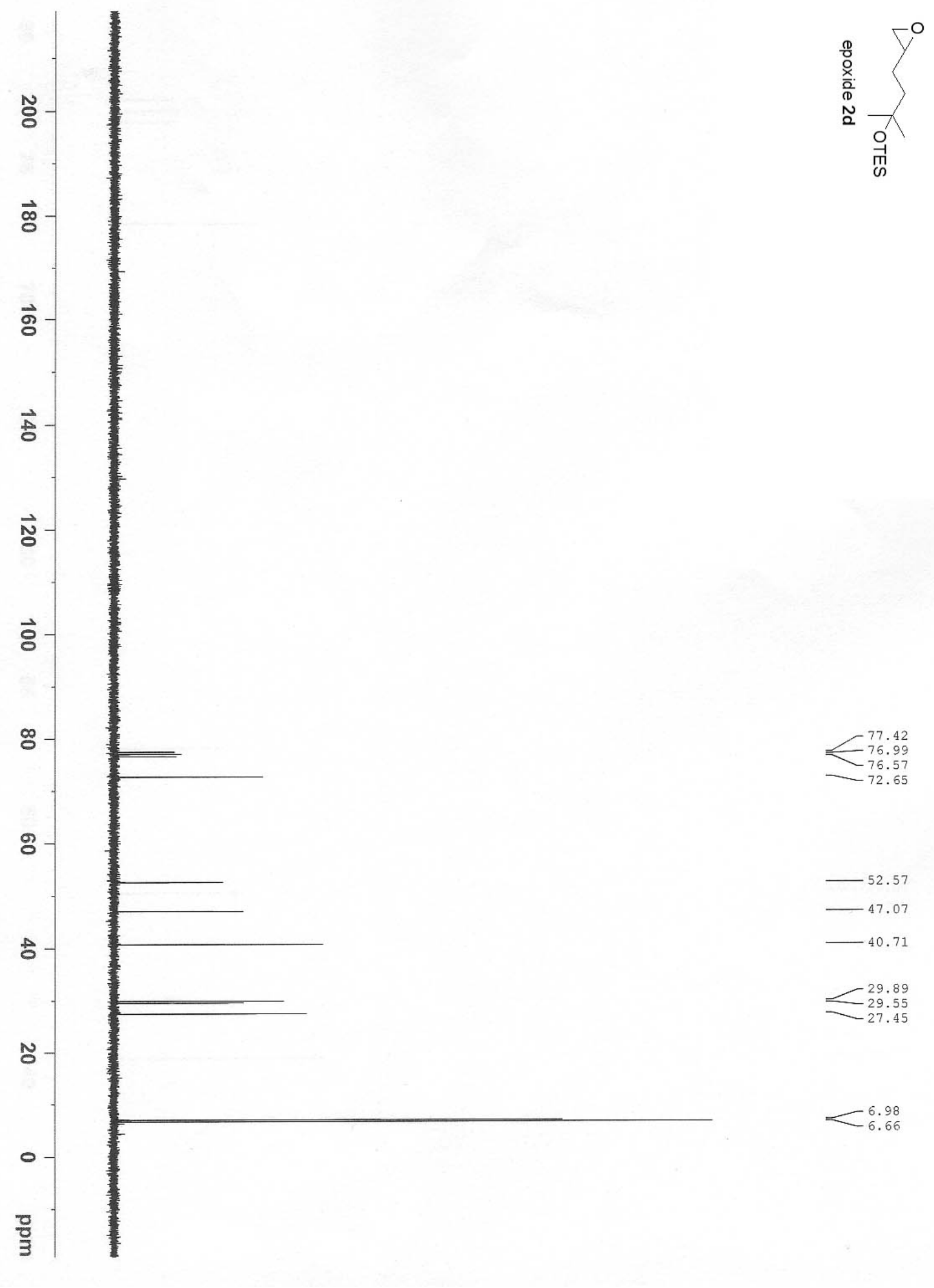

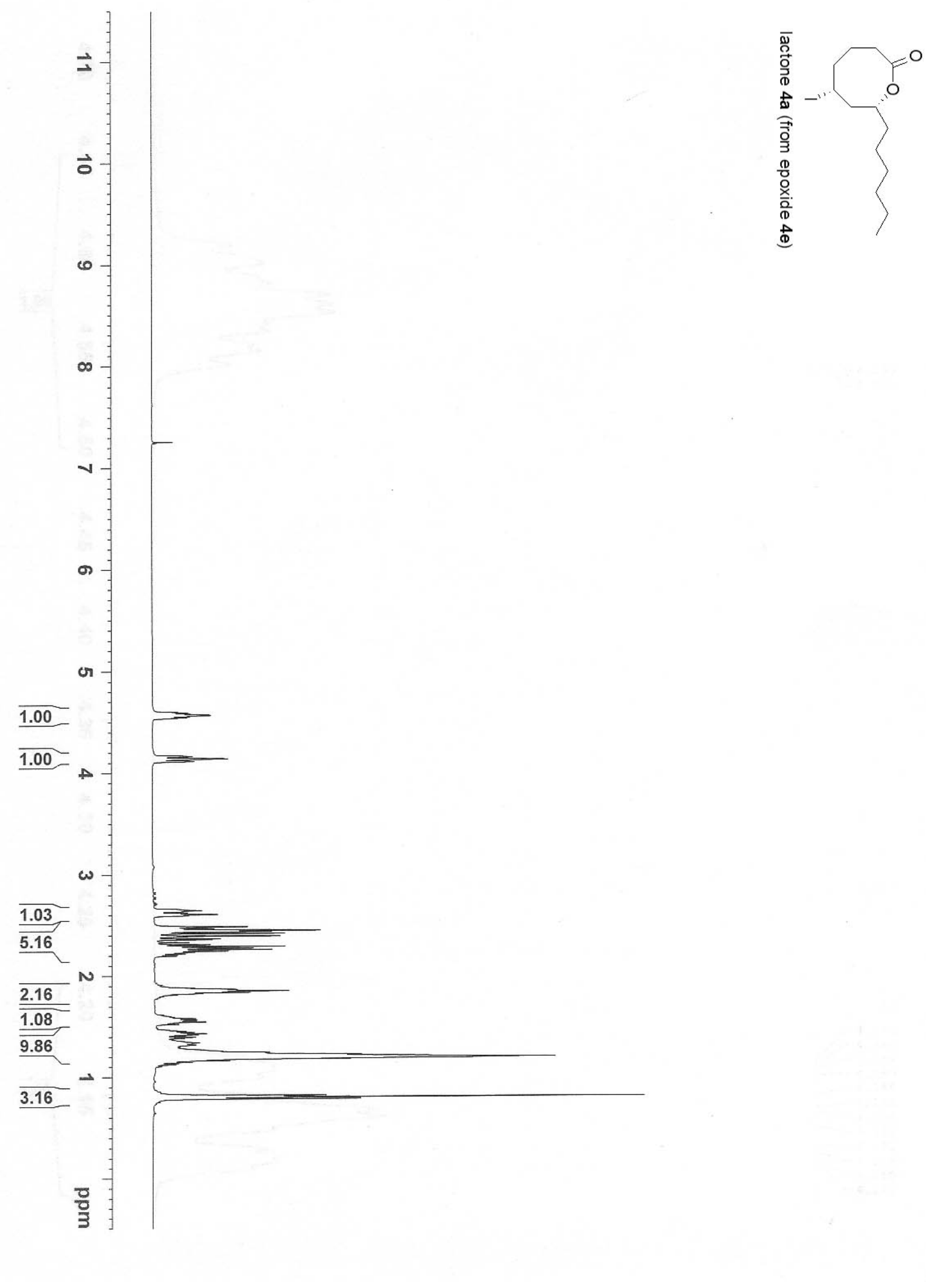

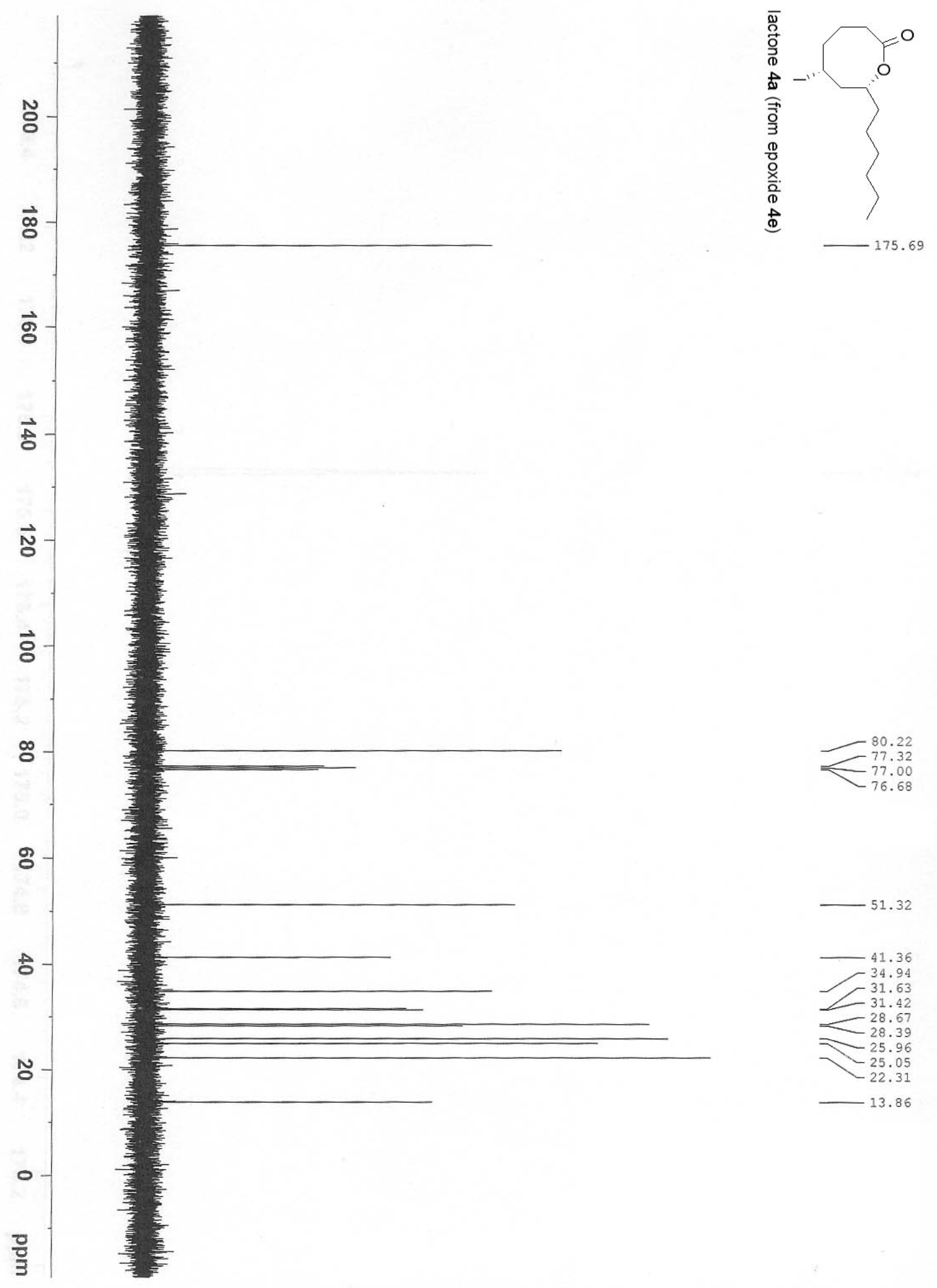

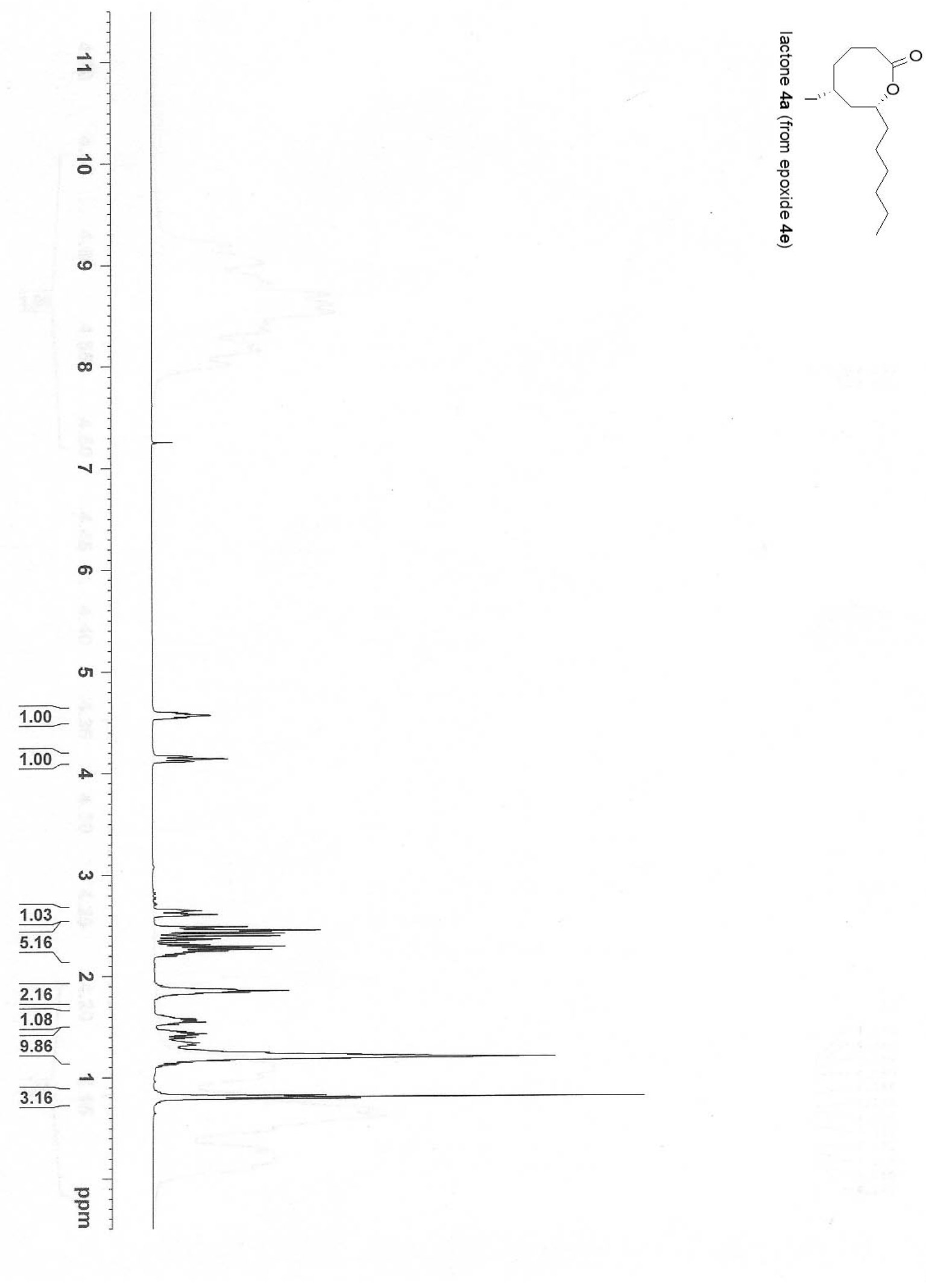


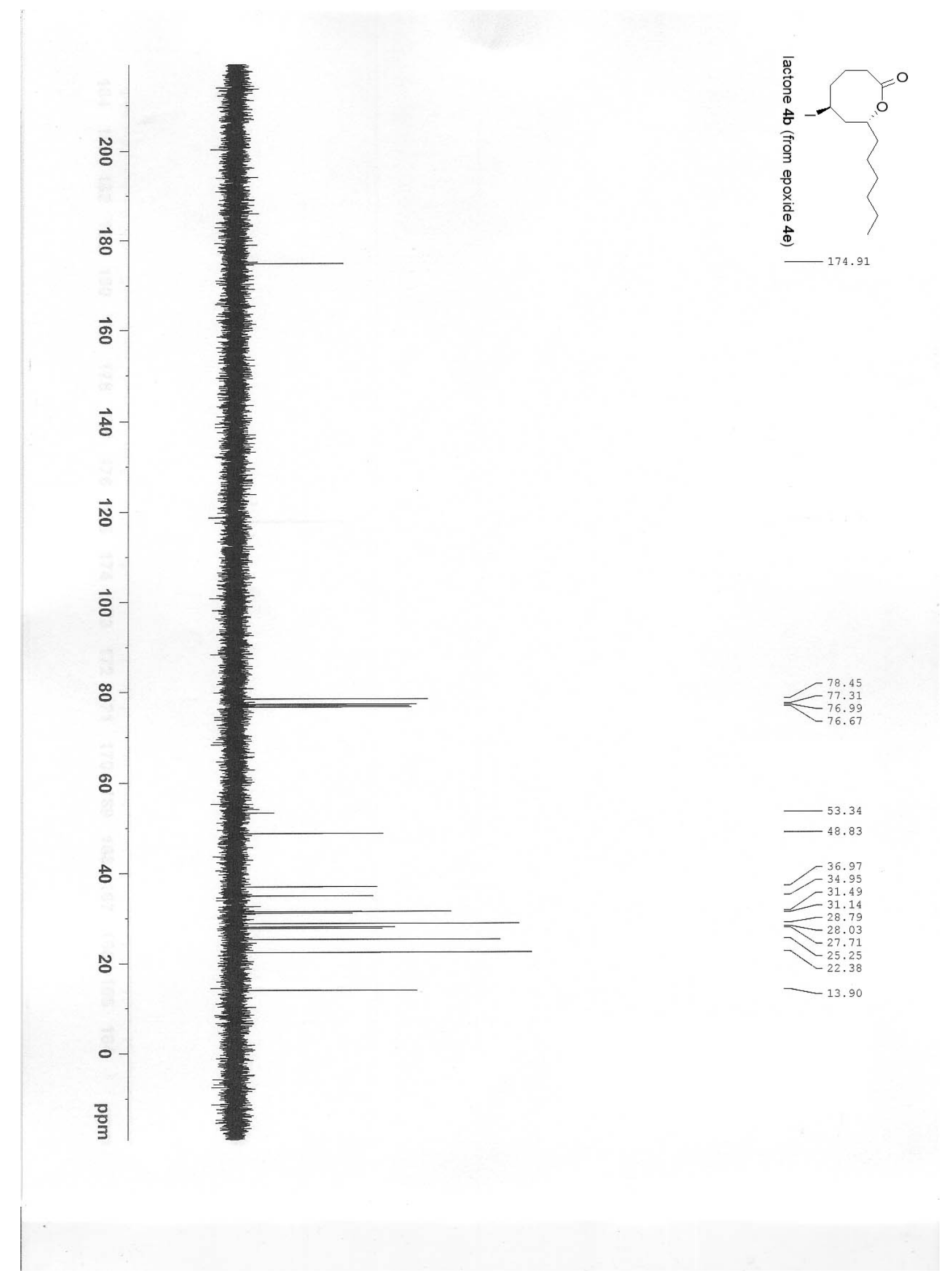



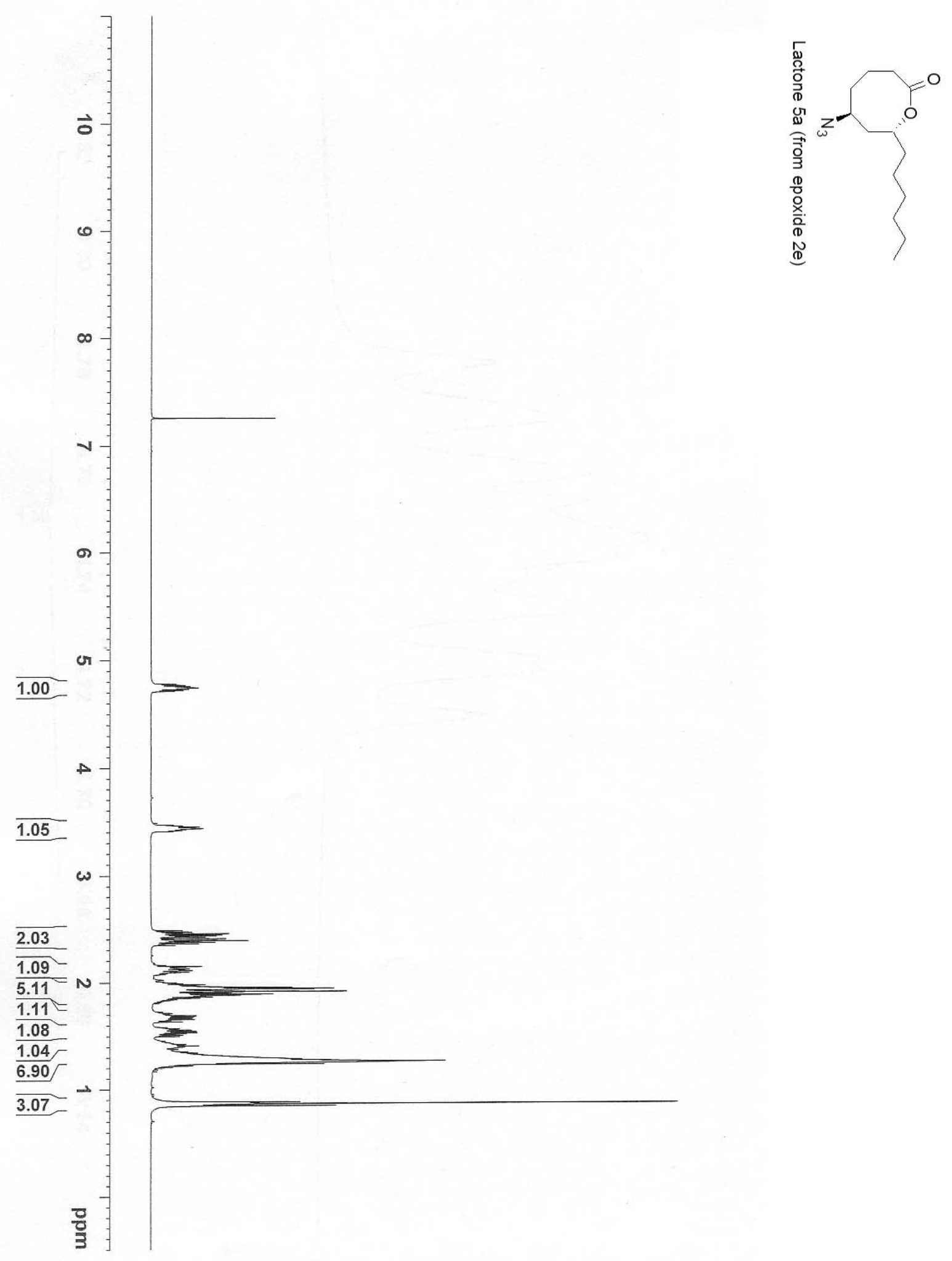

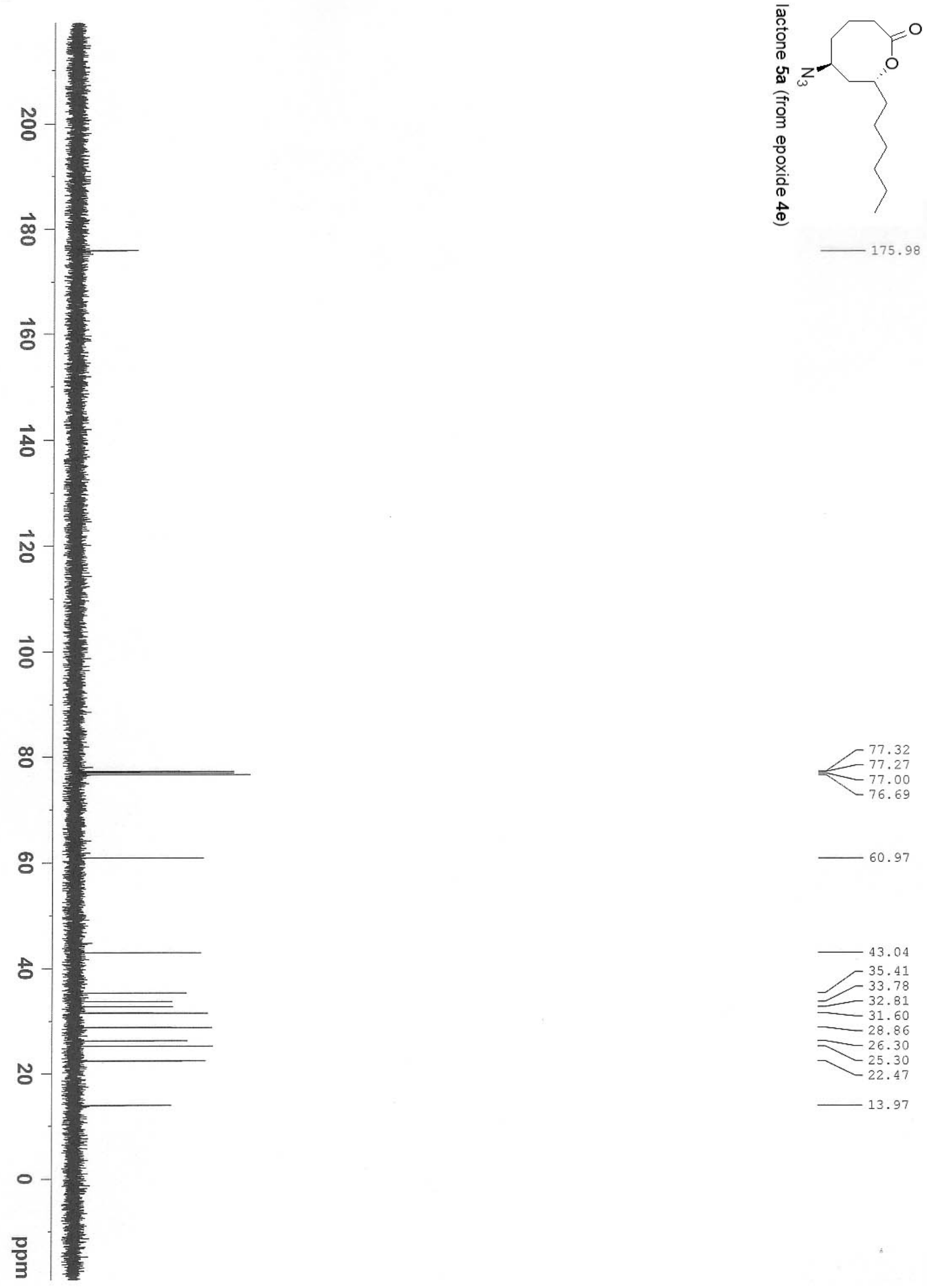

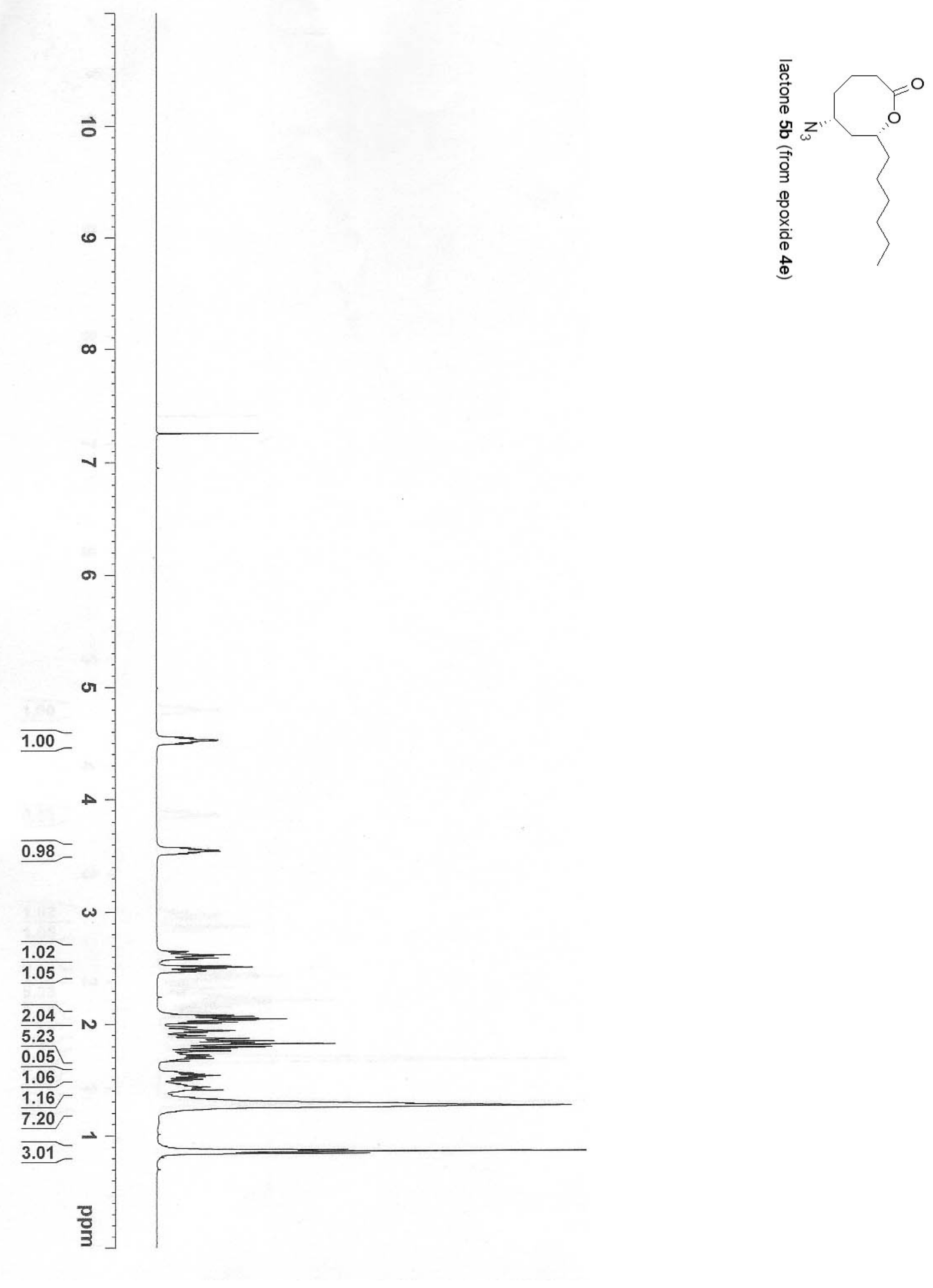

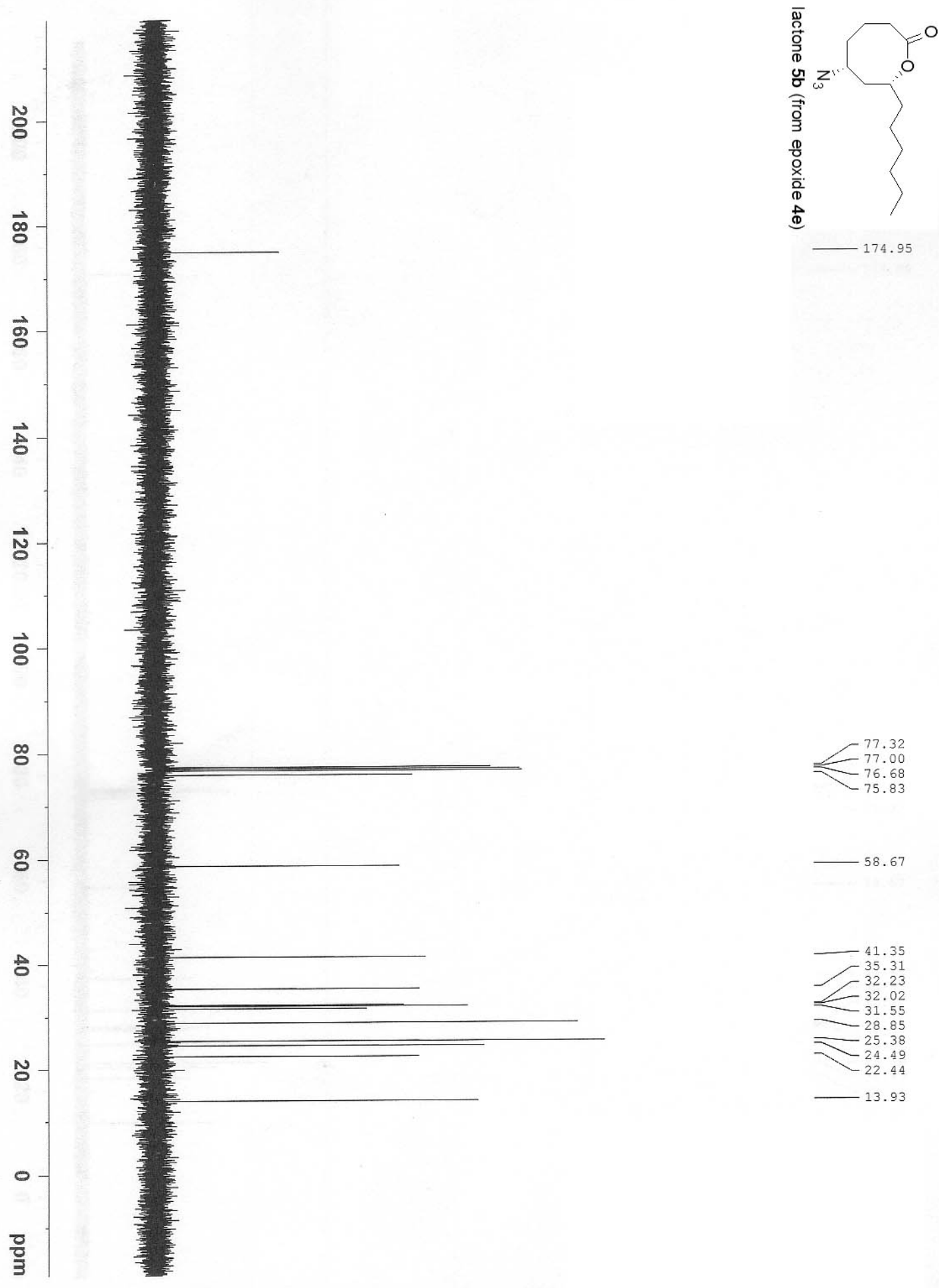

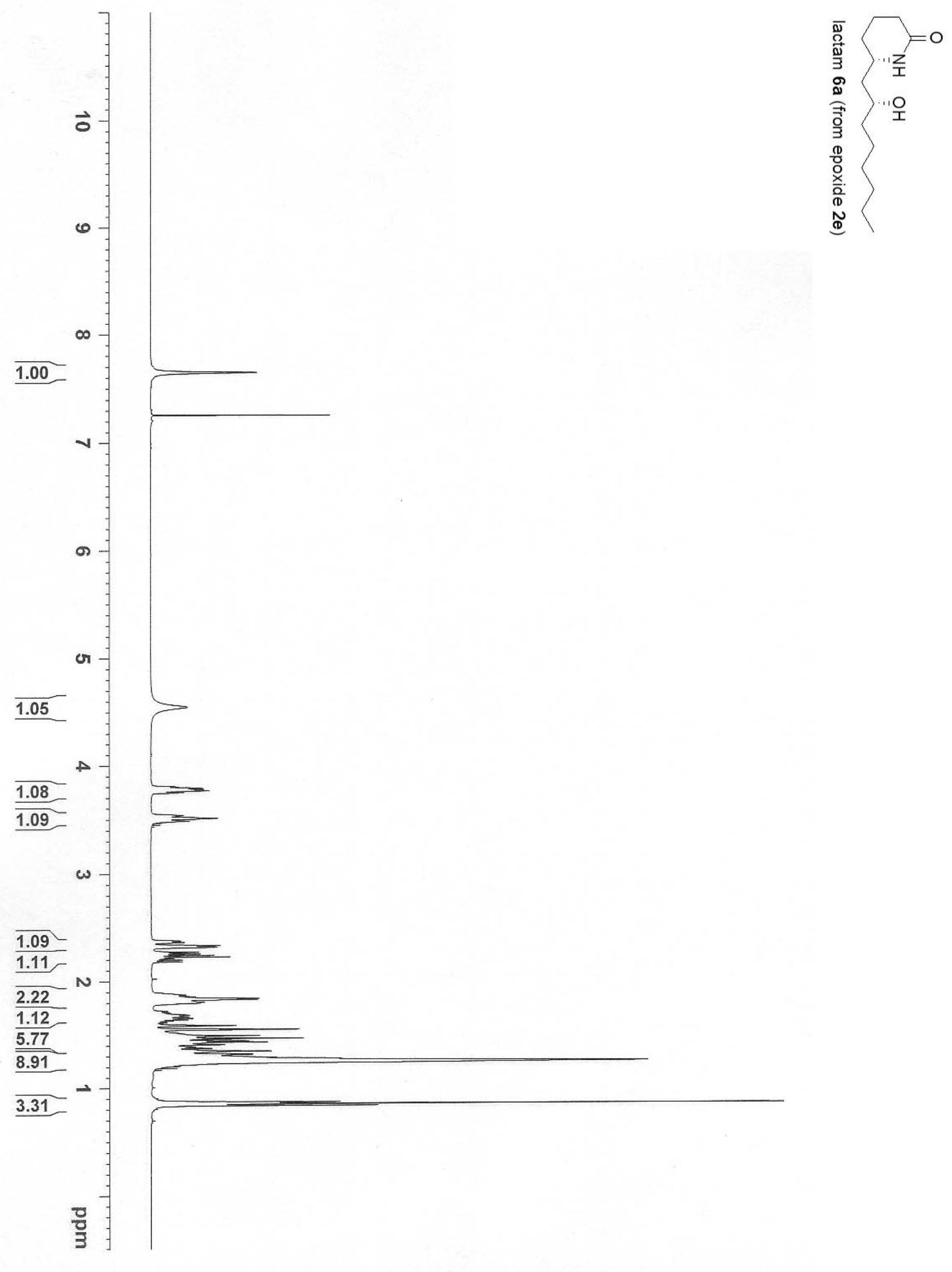

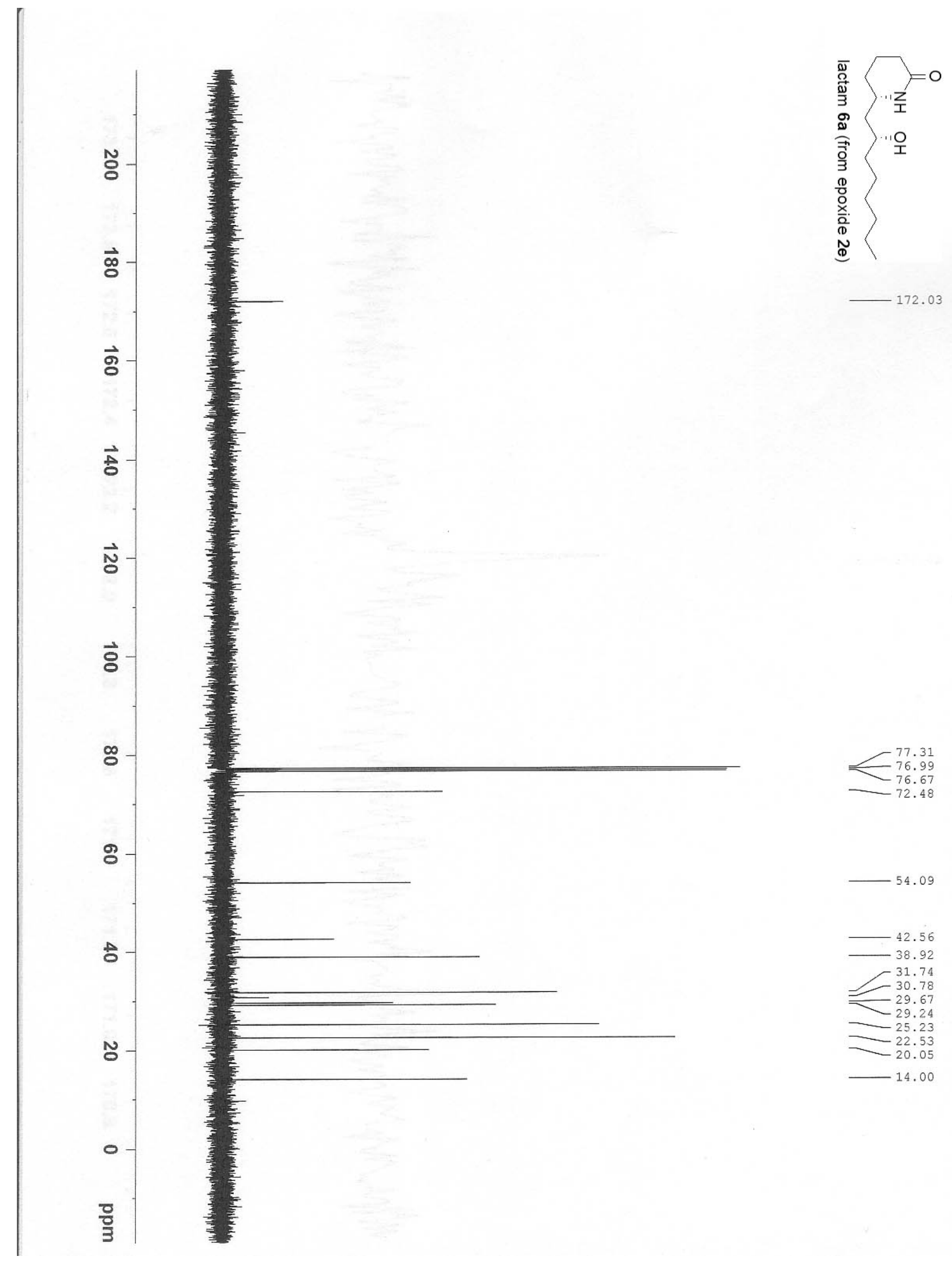


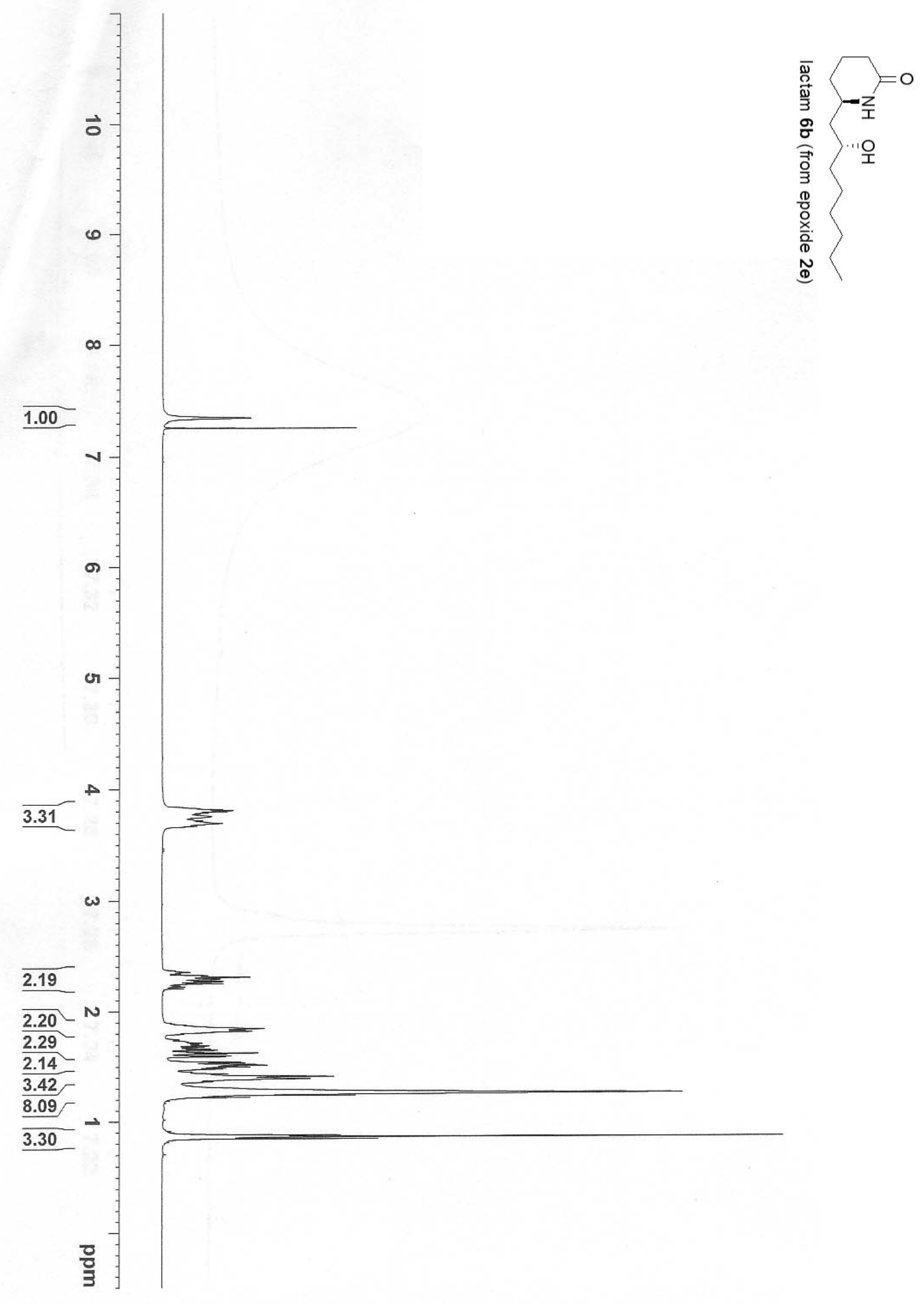



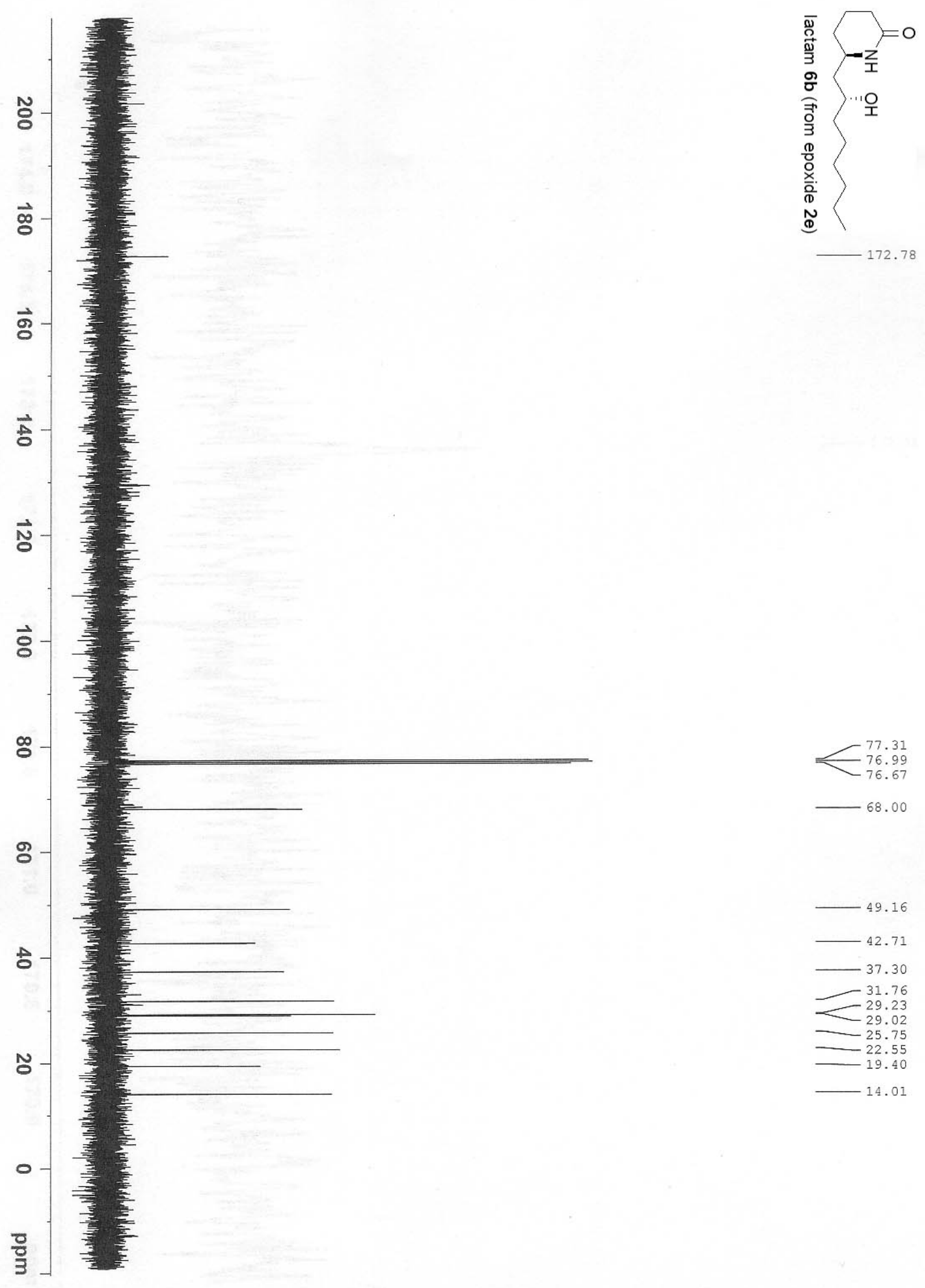

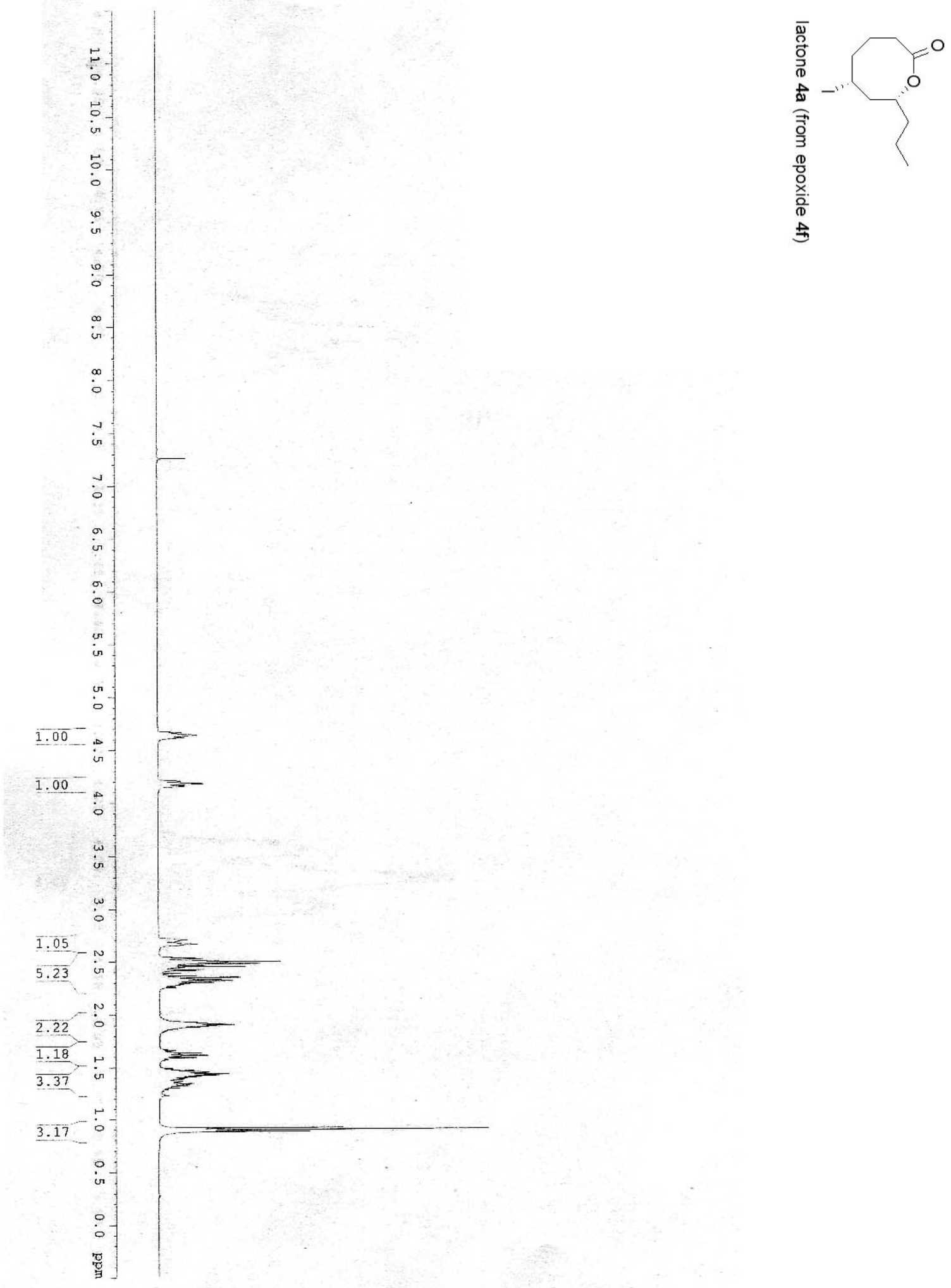

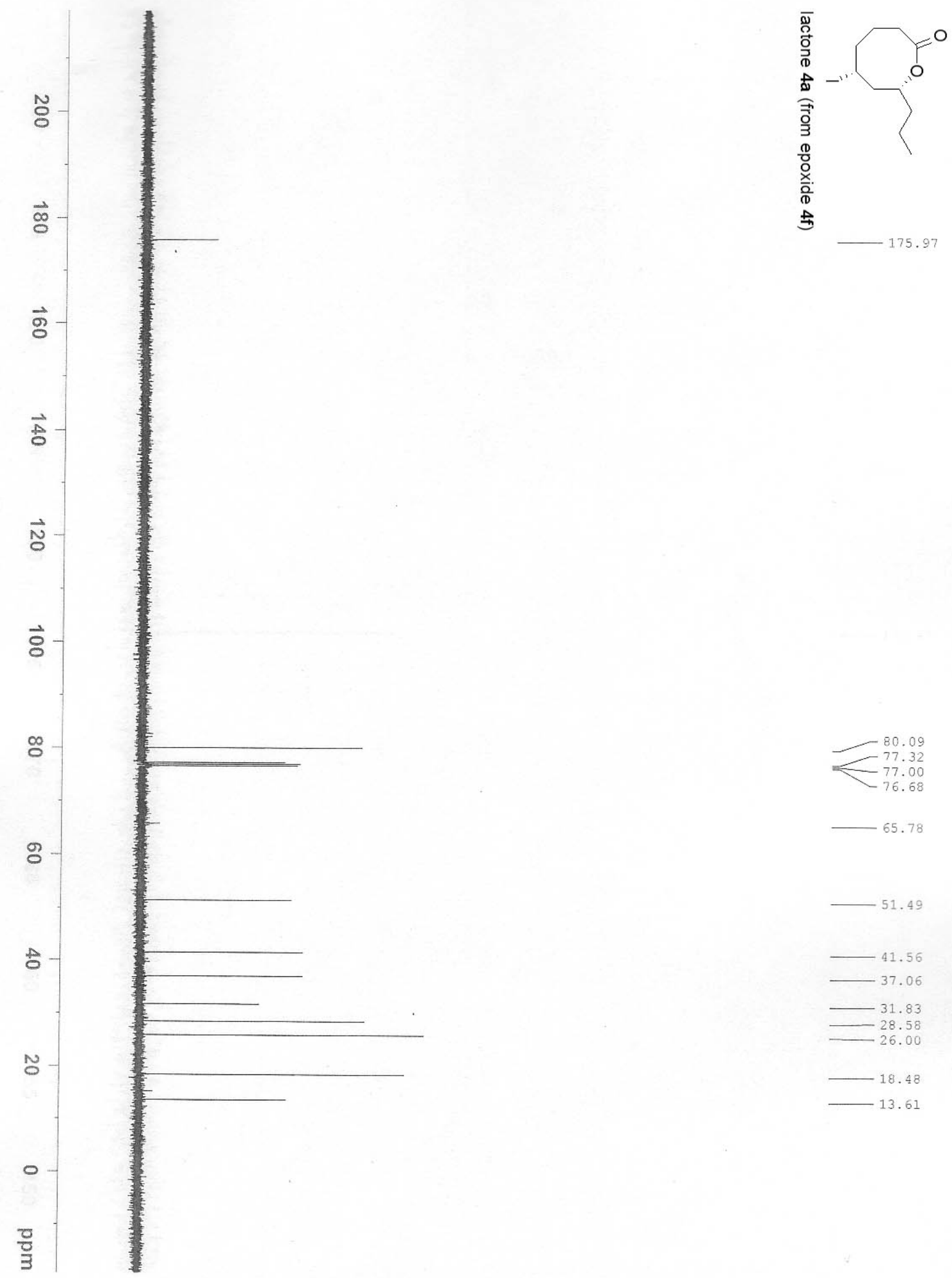

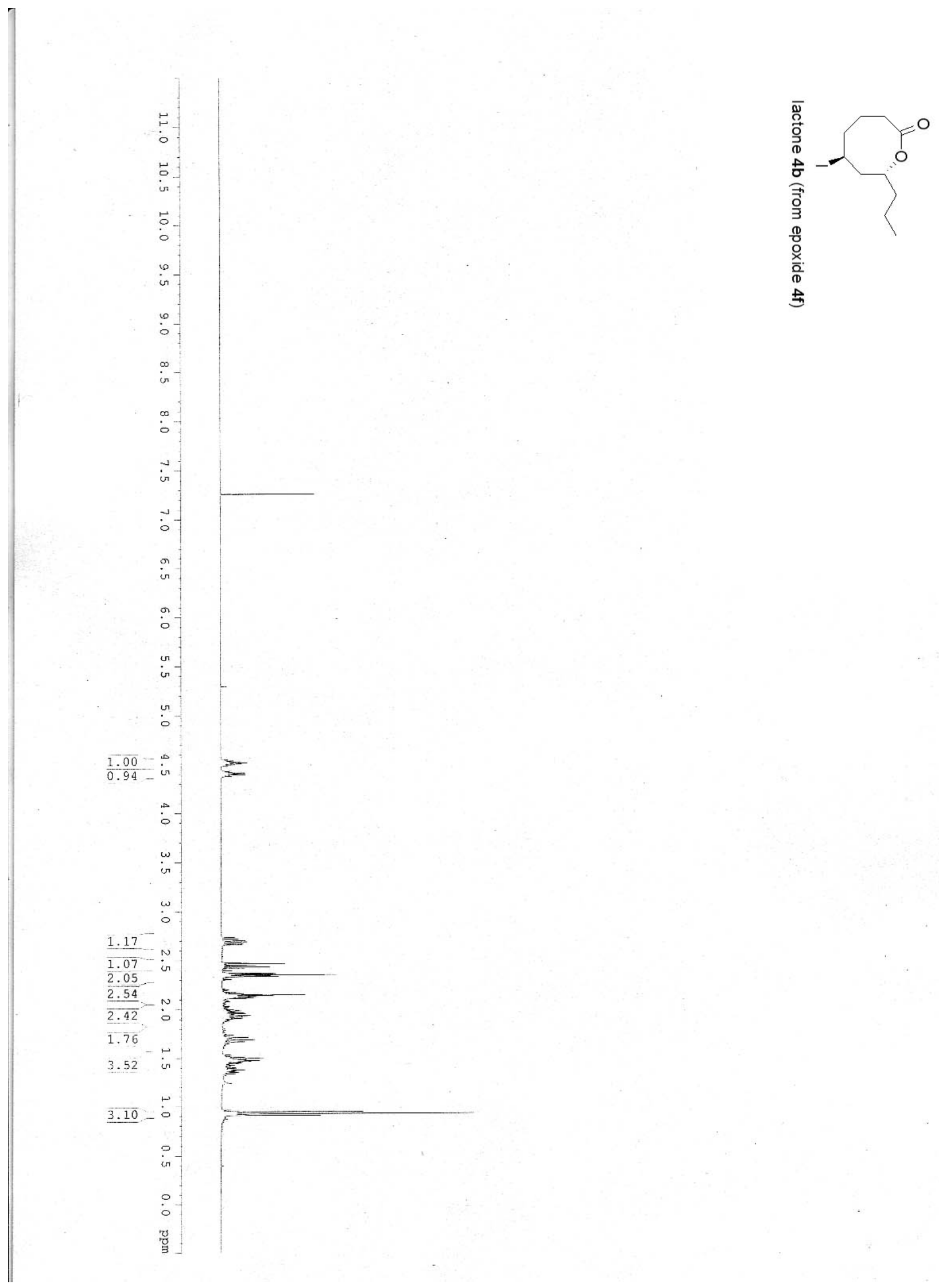

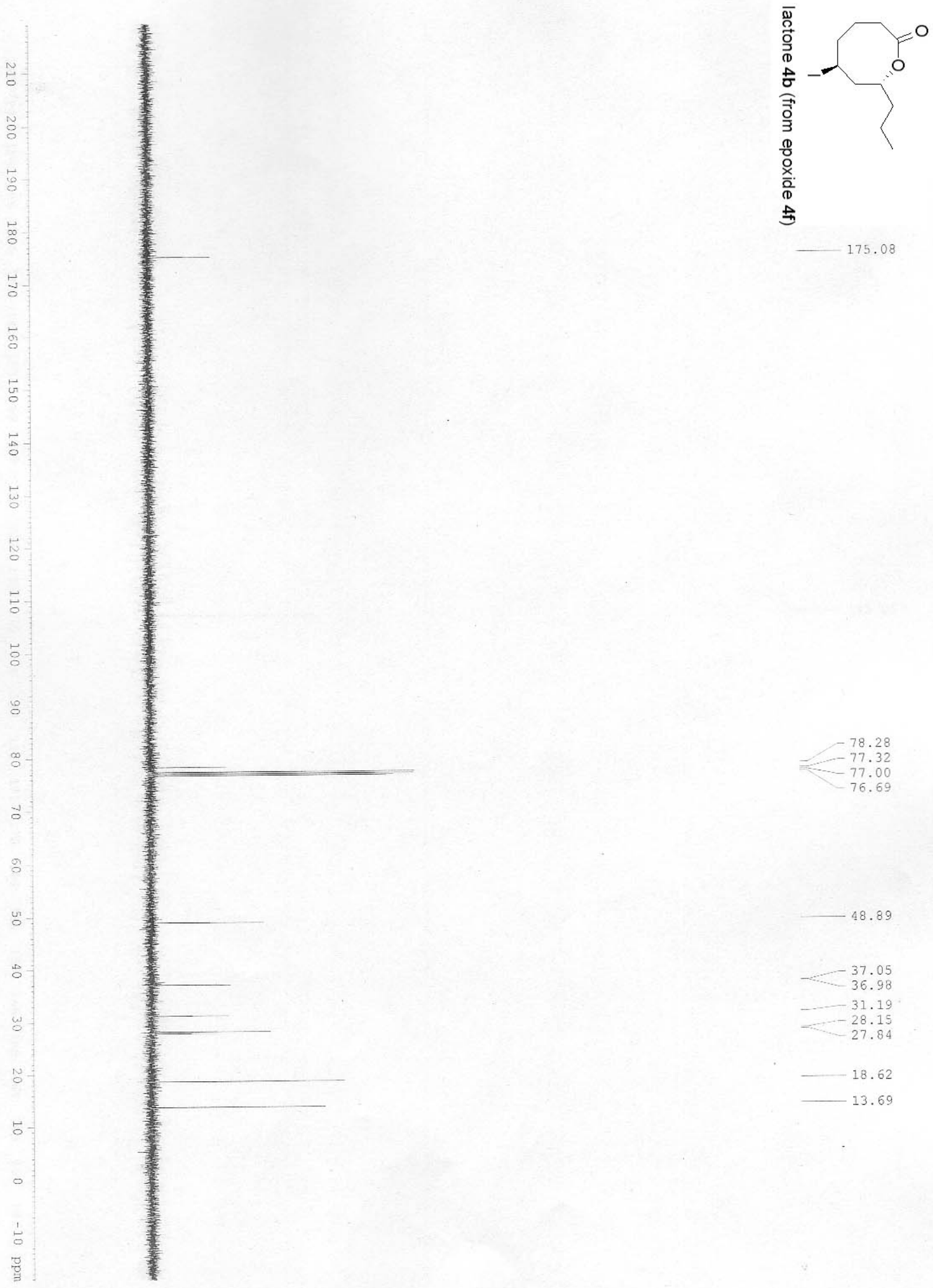

$-18.62$

13.69 

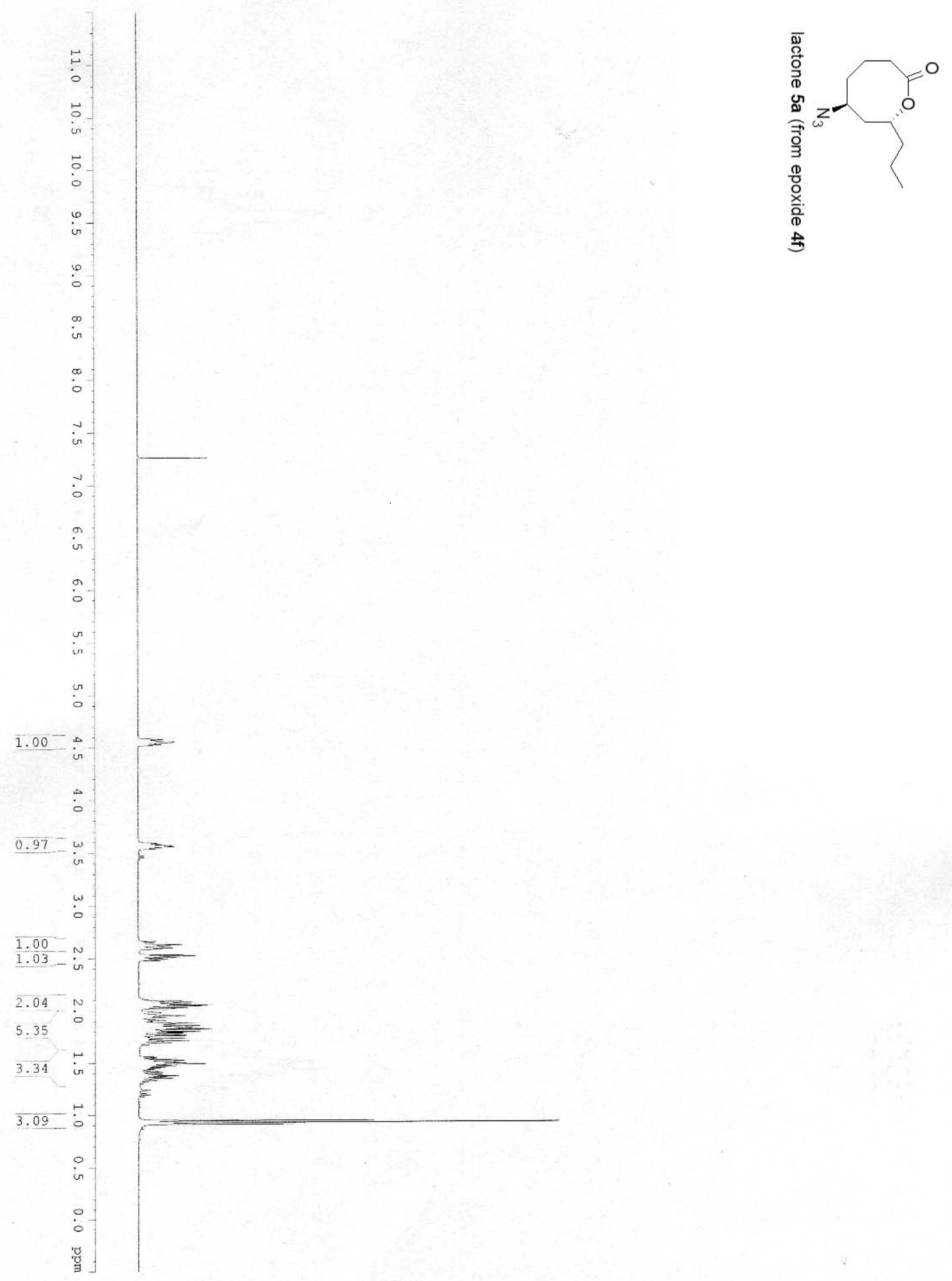

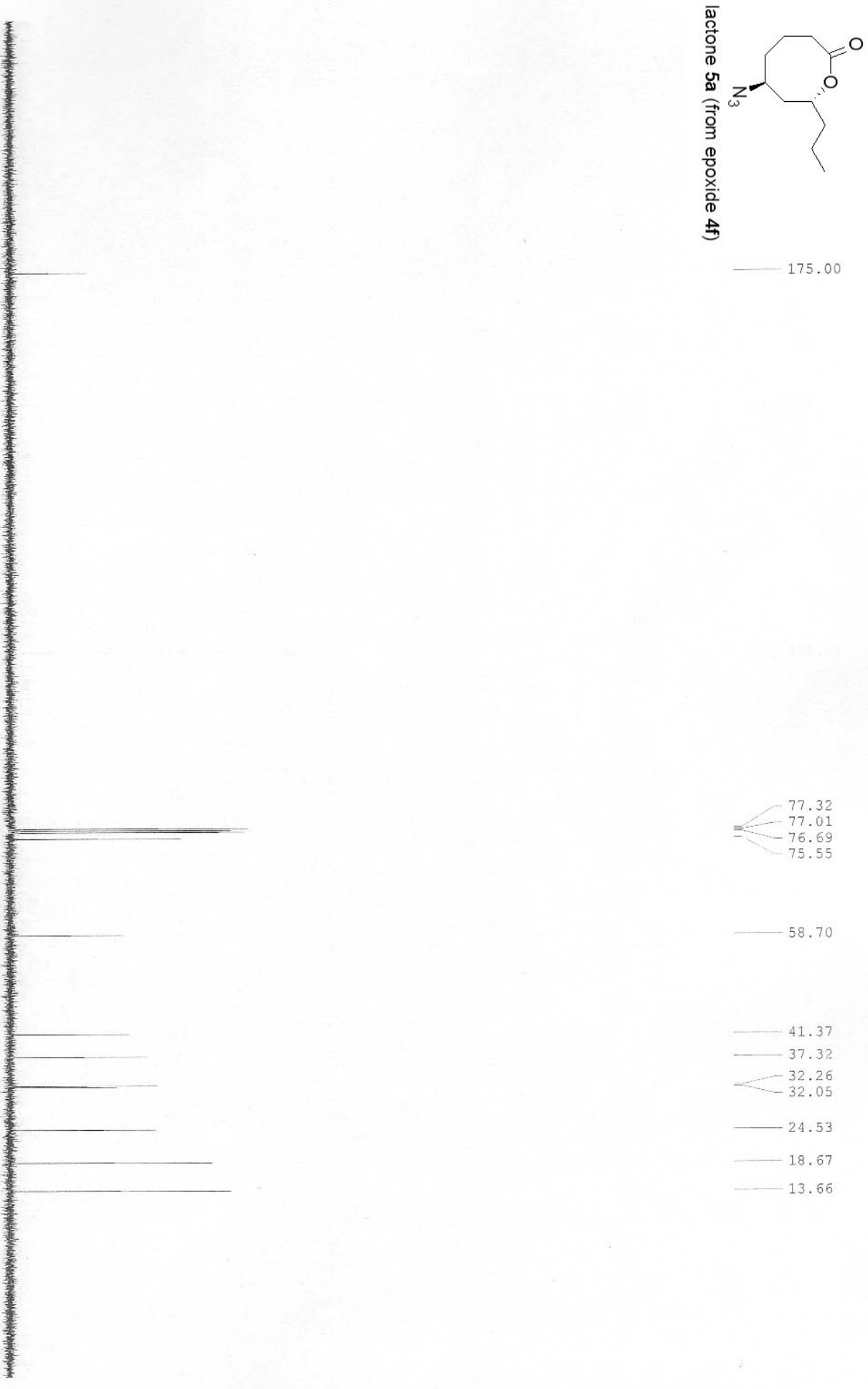

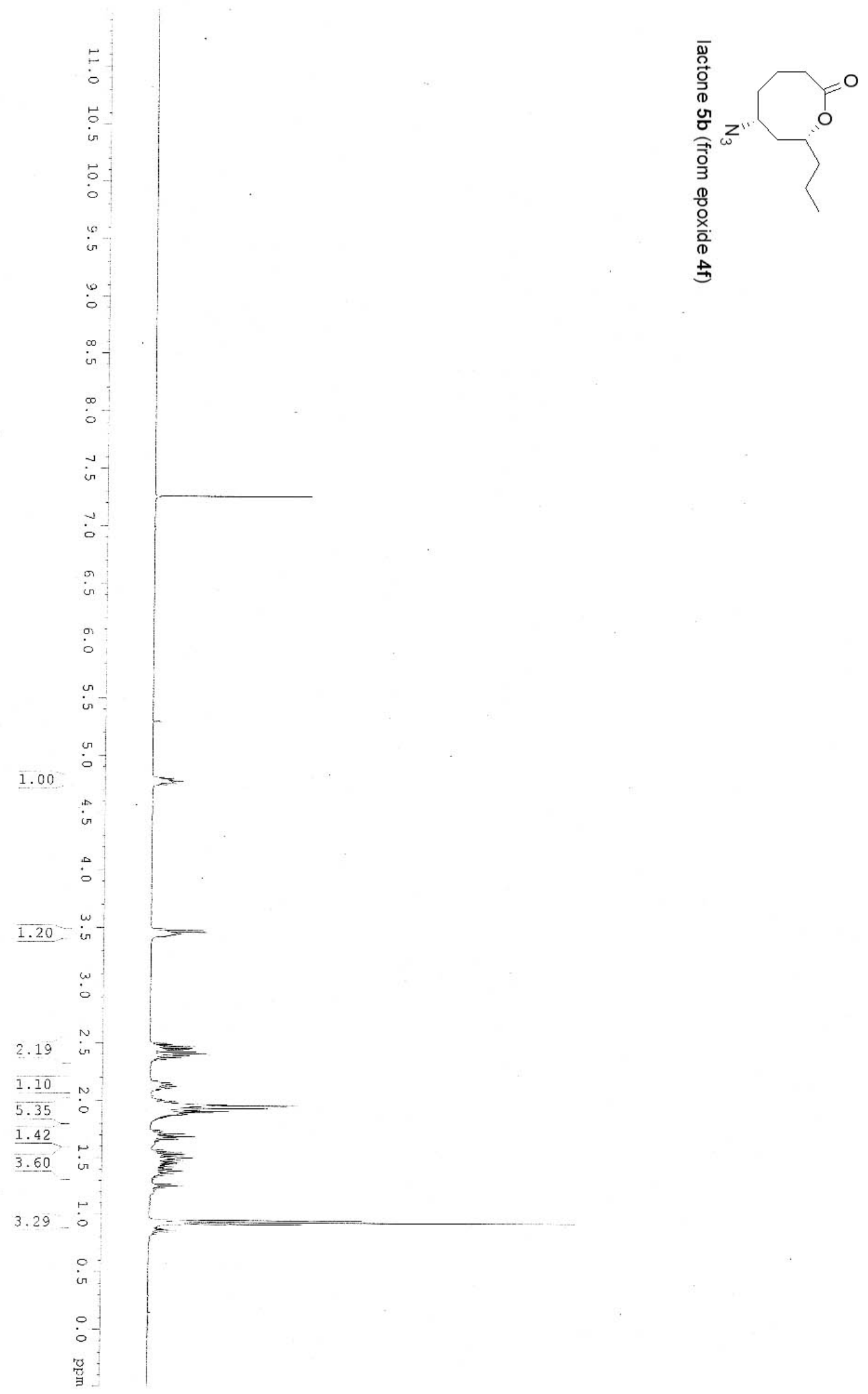


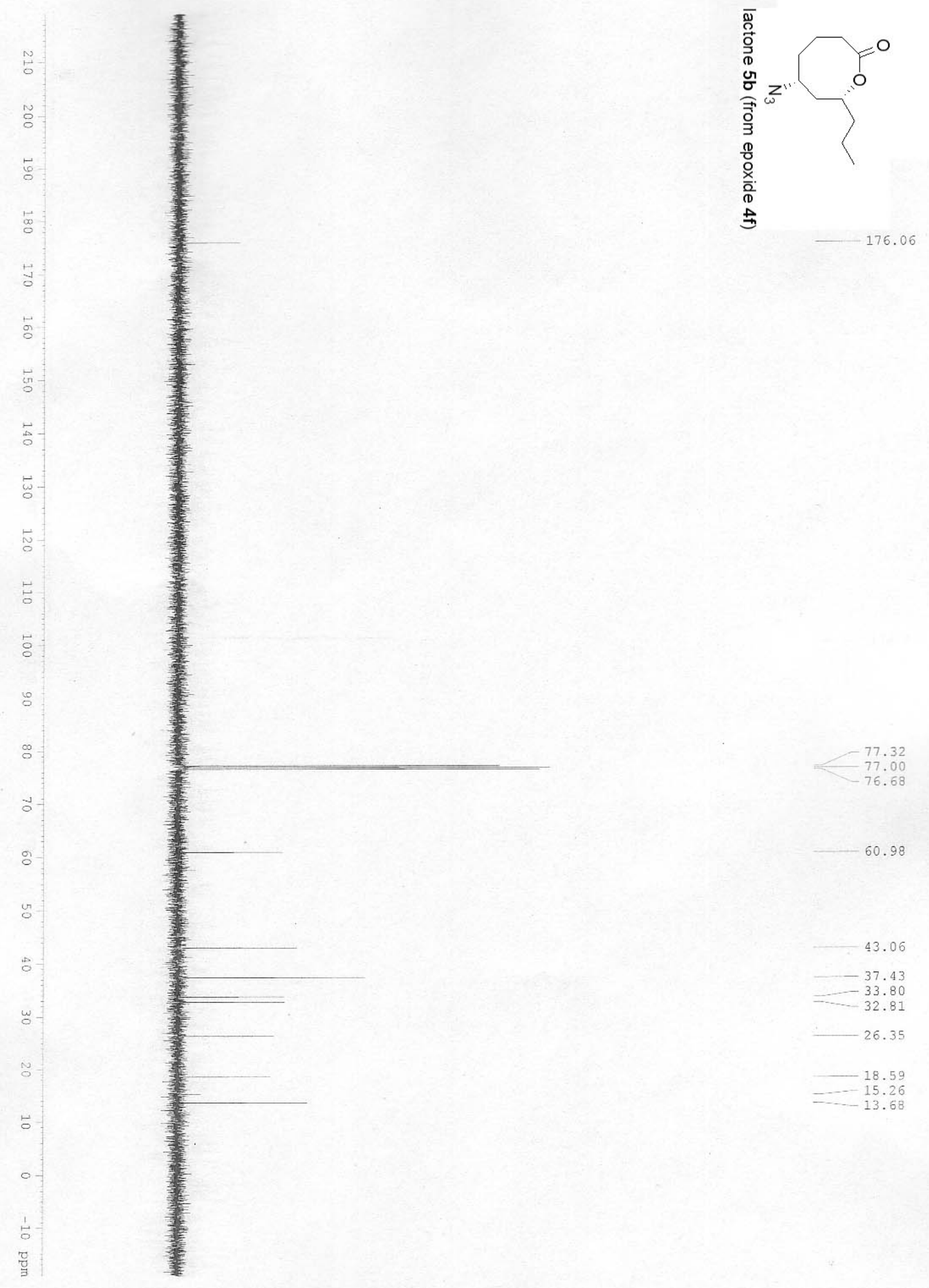

86 

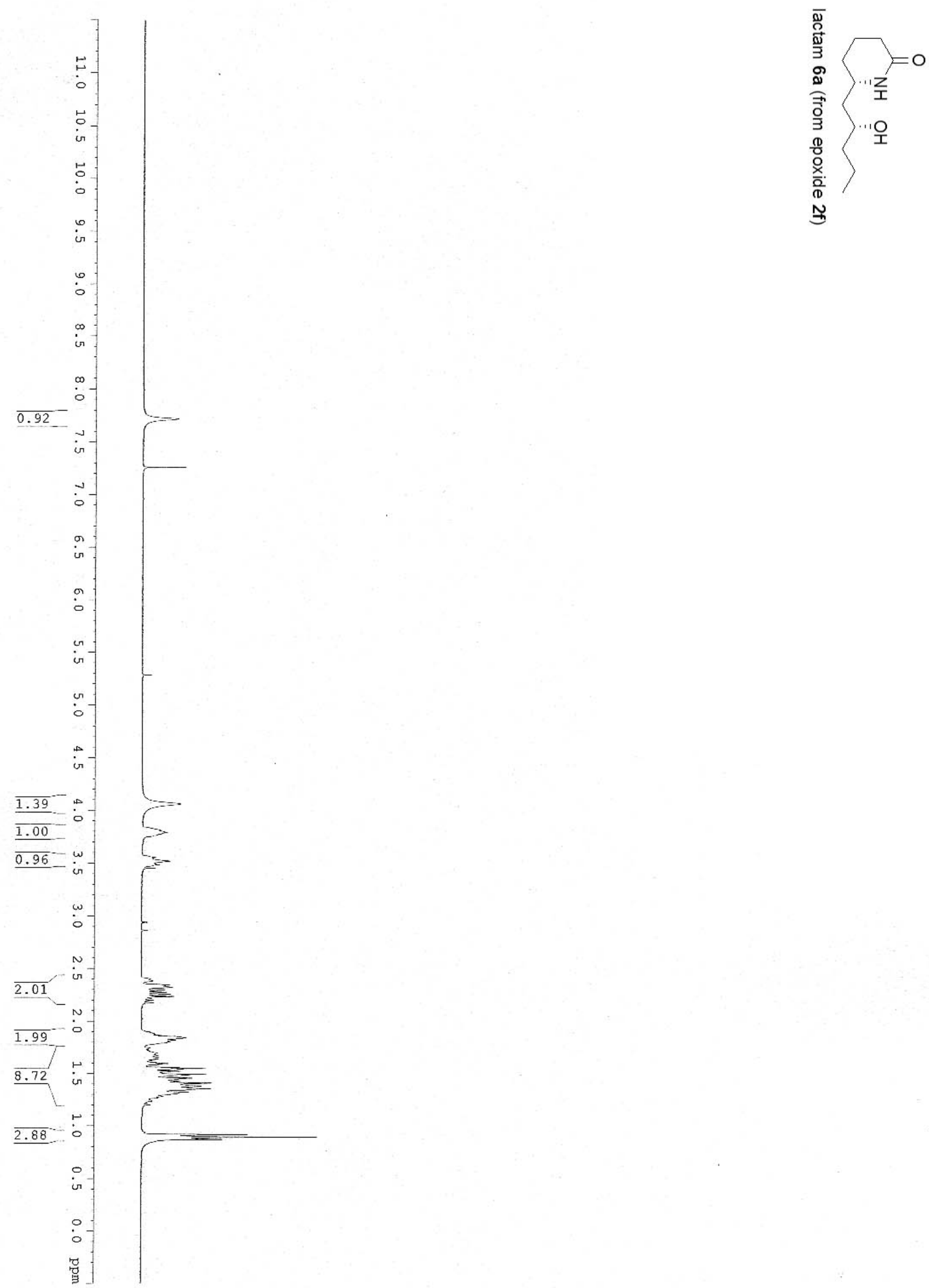


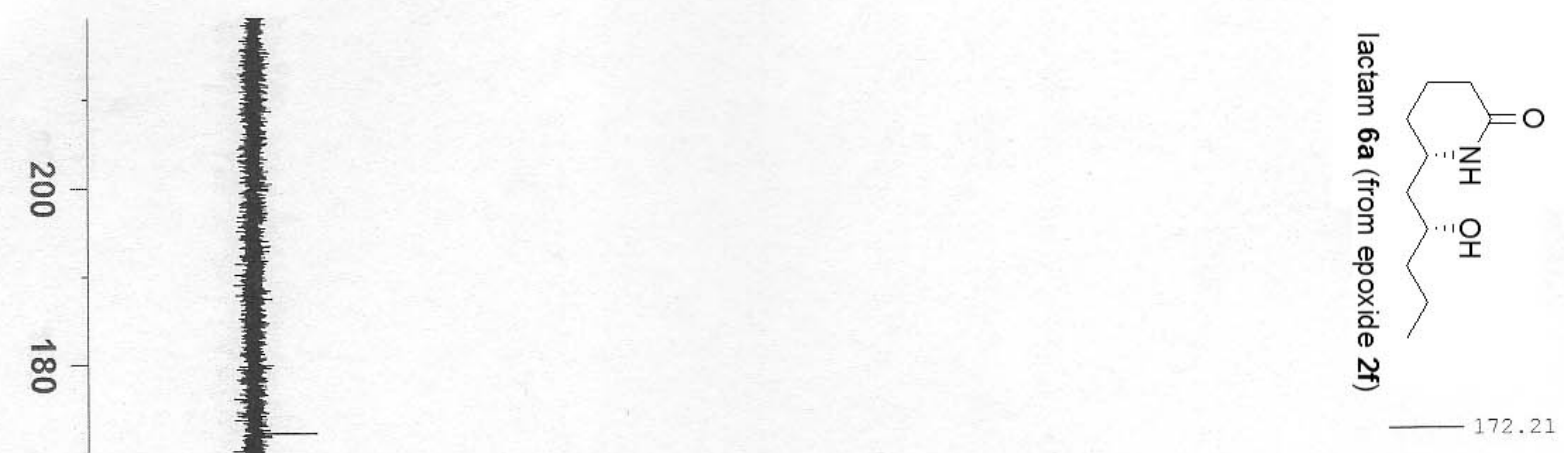

홍

$\overrightarrow{\hat{\theta}}$

$\vec{N}$

$\overrightarrow{8}$

ஃ

\&

o

N

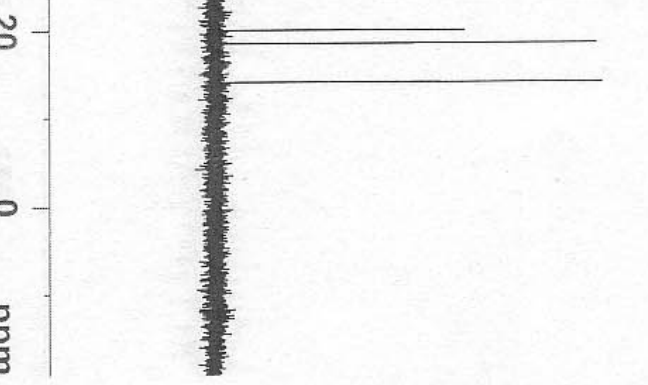

$-77.43$

76.5
-72.28

$-72.28$

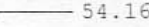

$=42.49$

$=30.70$

$-20.02$ 

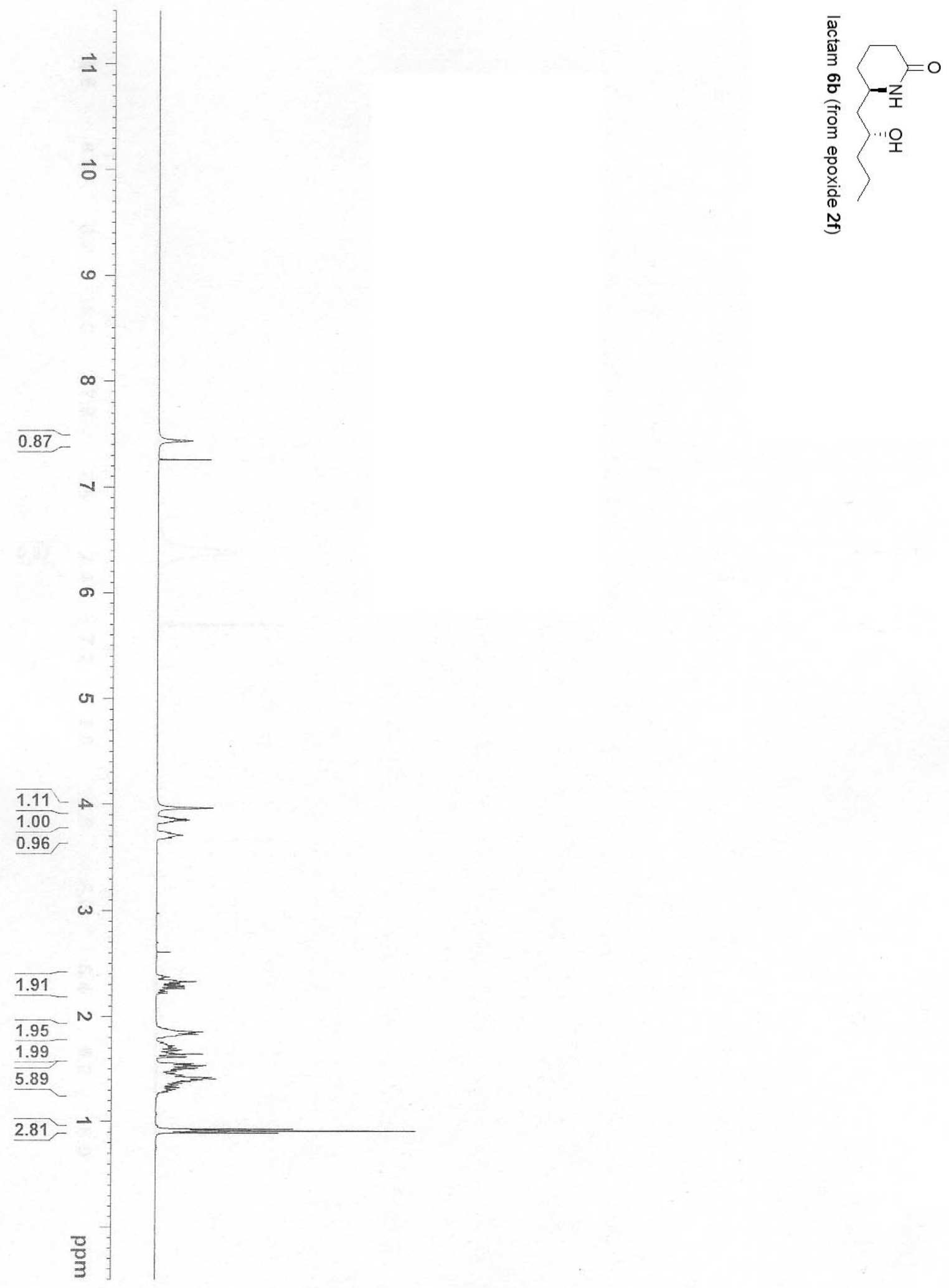


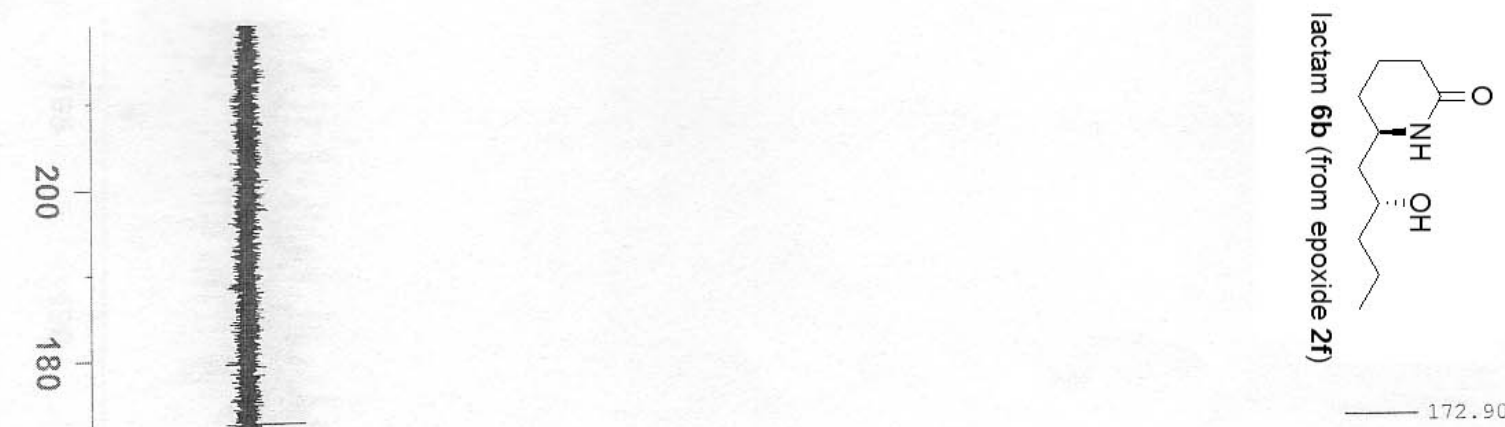

홍

$\overrightarrow{\text { ह }}$

$\vec{N}$

$\vec{\circ}$

$\stackrel{\infty}{\circ}$

$\vec{\circ}$

8

of

ก

$\circ$

흥
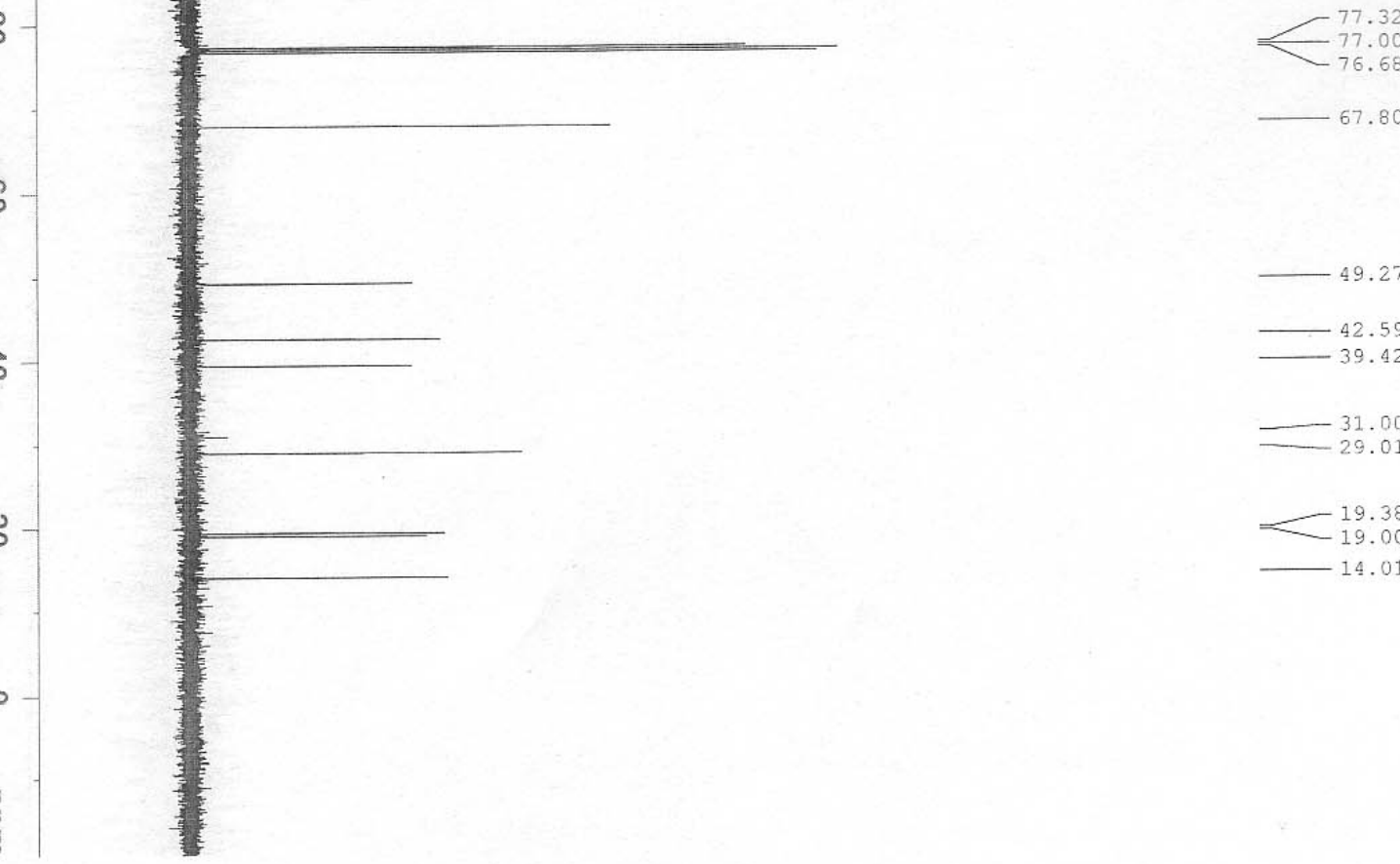

$-49.27$

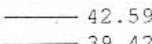

$-31.00$

$+19.38$ 

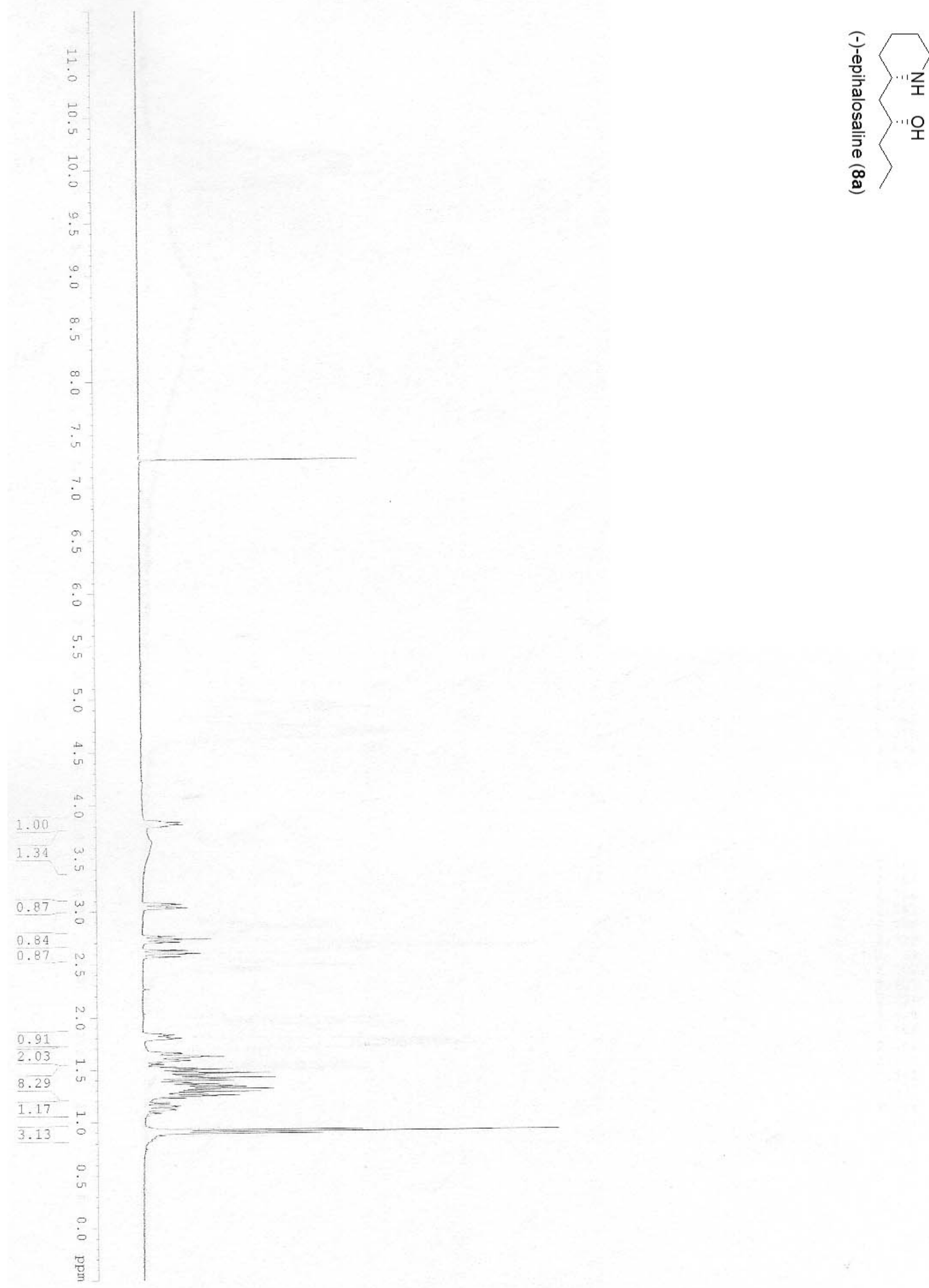

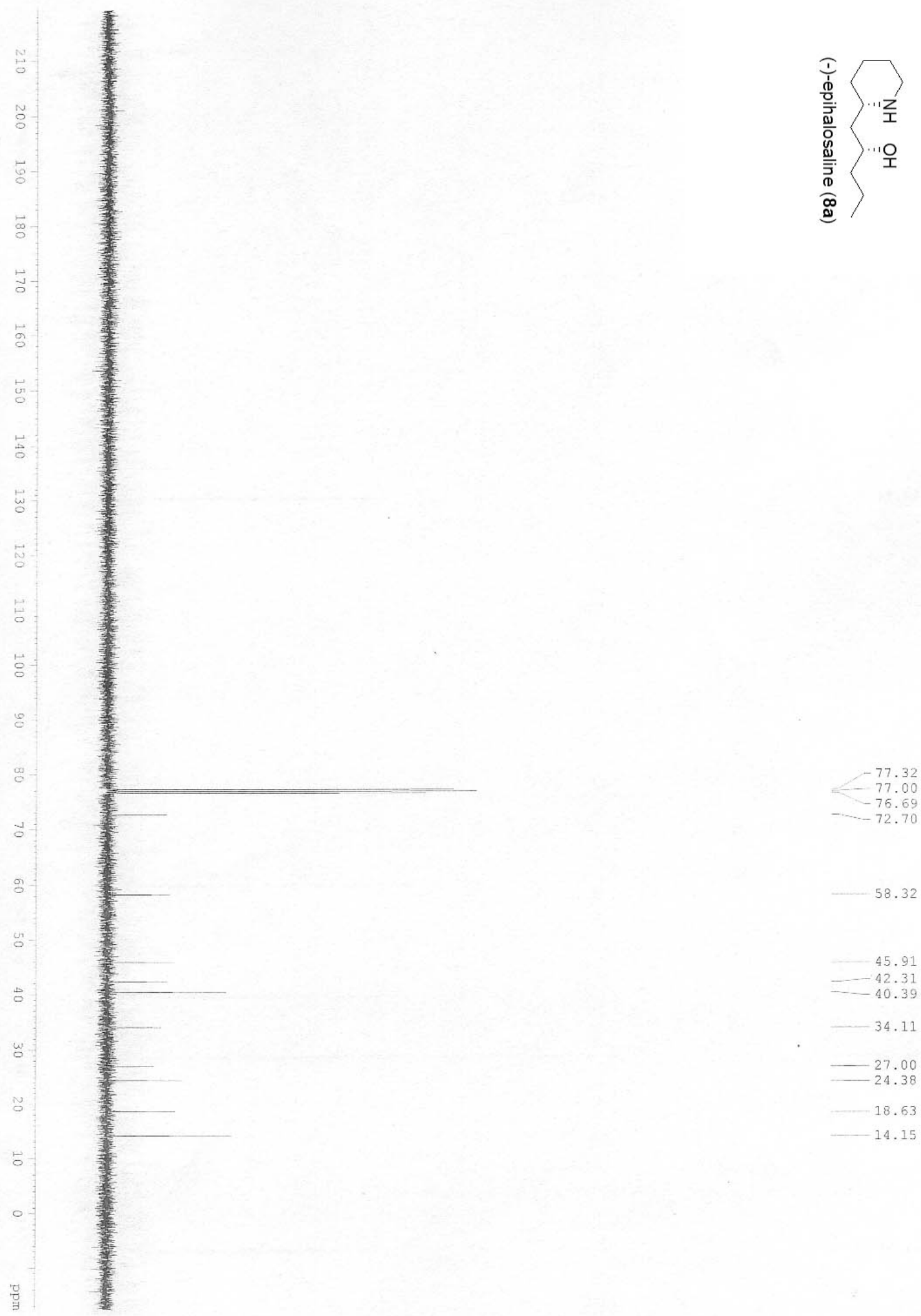

$-58.32$

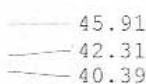

$-34.11$

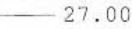

-27.00
-24.38

$-18.63$

$-14.15$ 

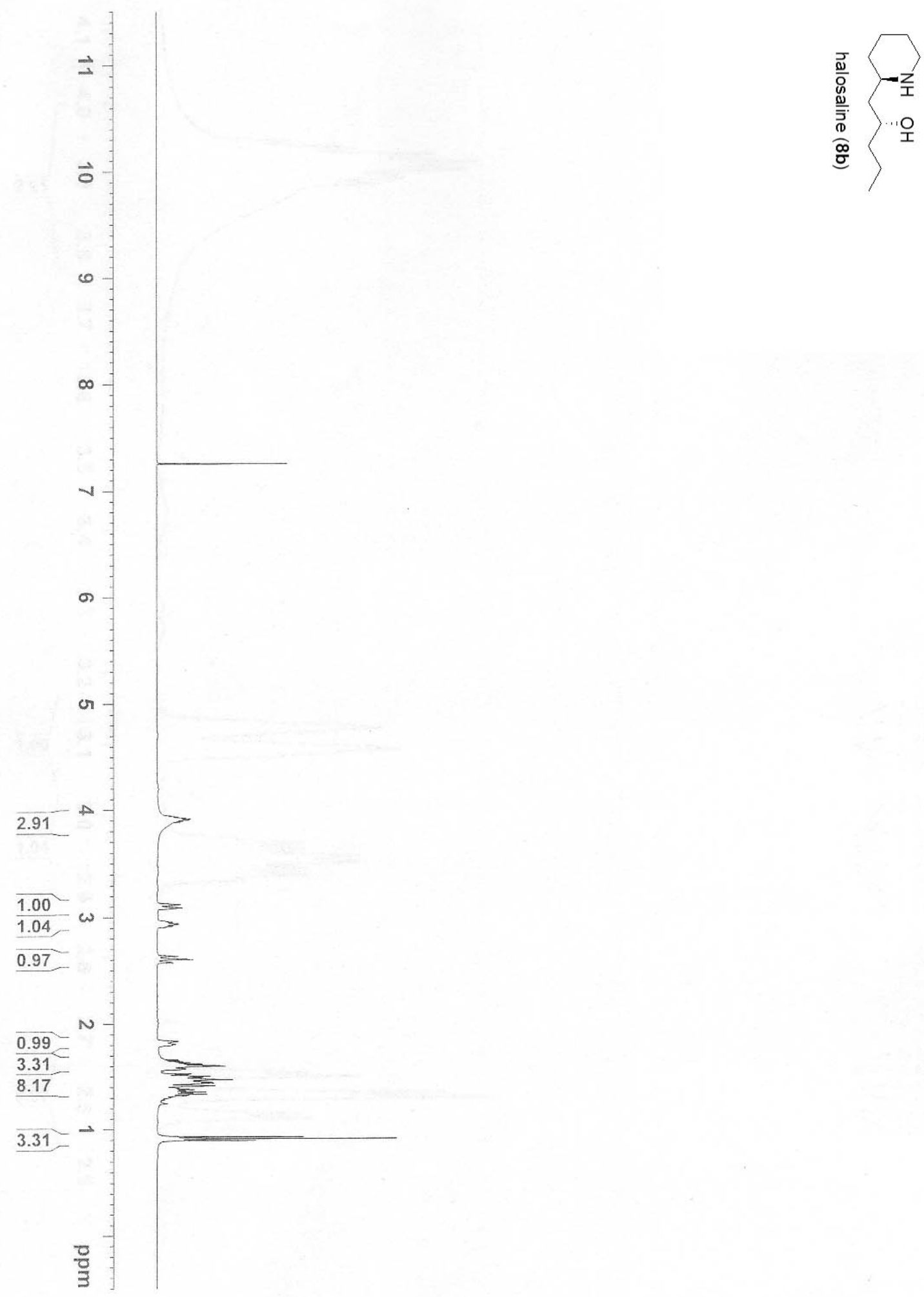

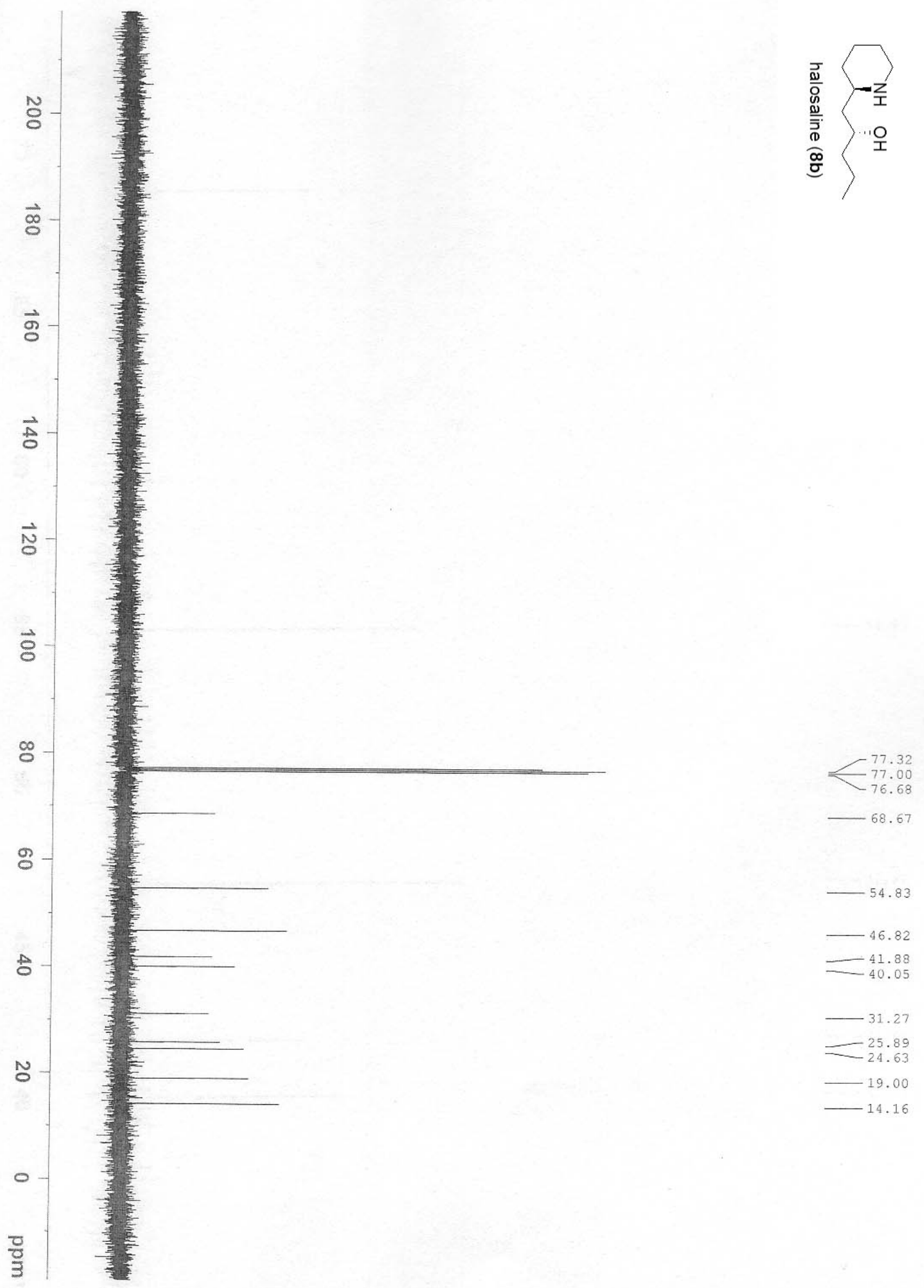

$-54.83$

$-46.82$

$-41.88$

$-31.27$

$-25.89$

$-19.00$

$-14.16$ 


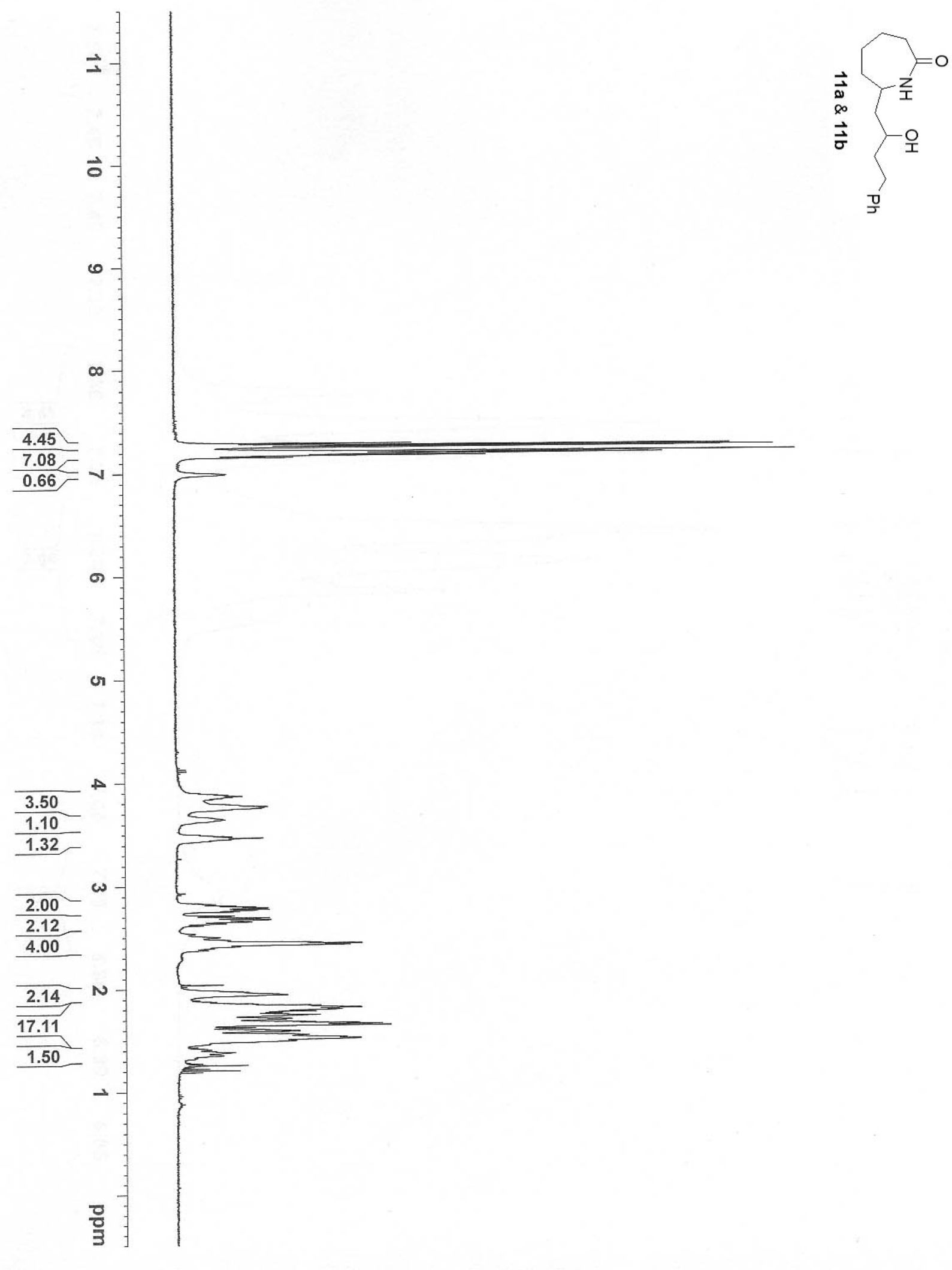



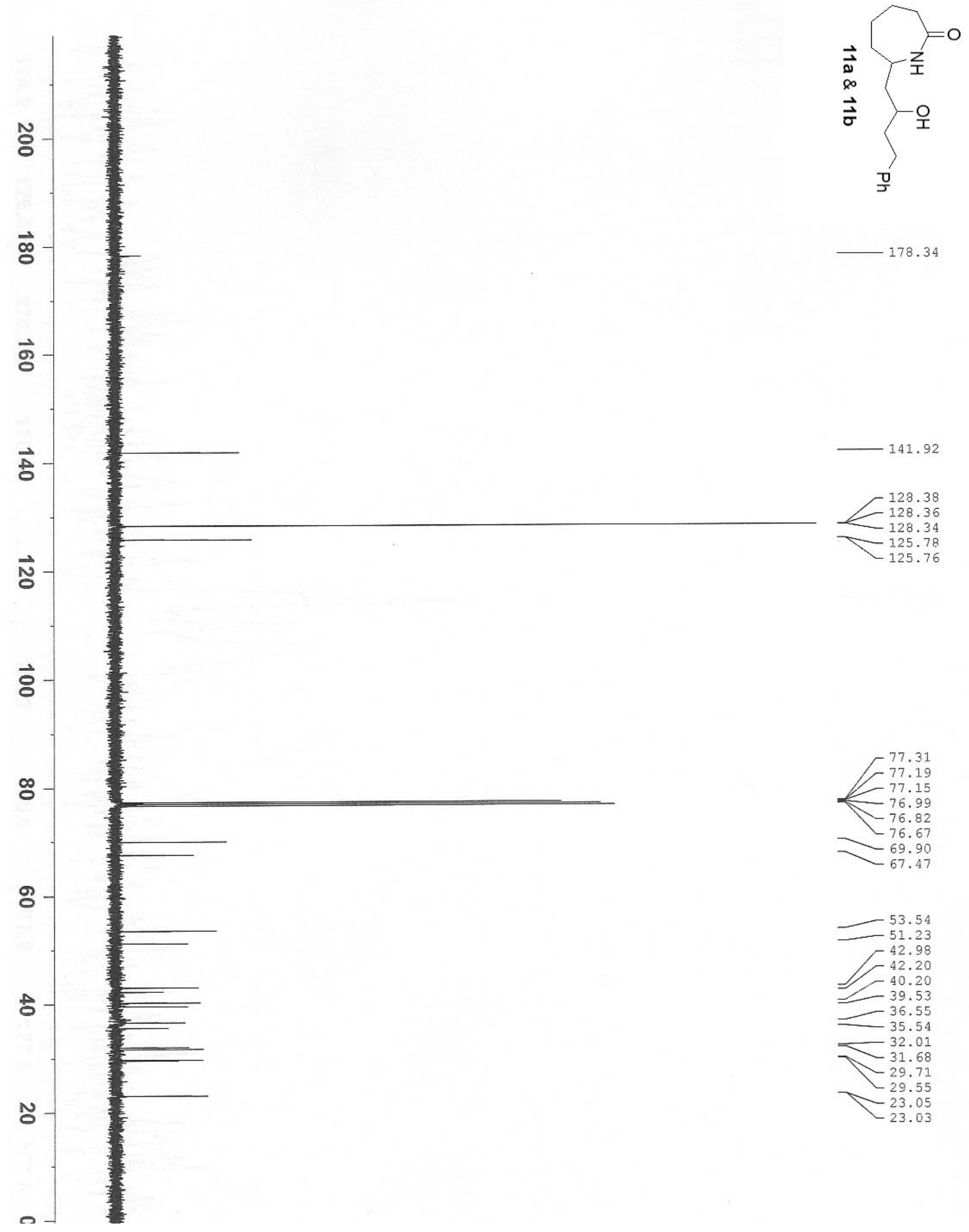

$-42.98$

42.20
-40.20
-49.53

$=-39.53$

$-36.55$

35.54

32.01

$-31.68$

$-29.71$

$\longrightarrow 23.05$

$-23.03$ 
III. X-Ray Structures:

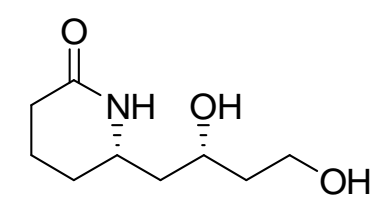

7a, syn

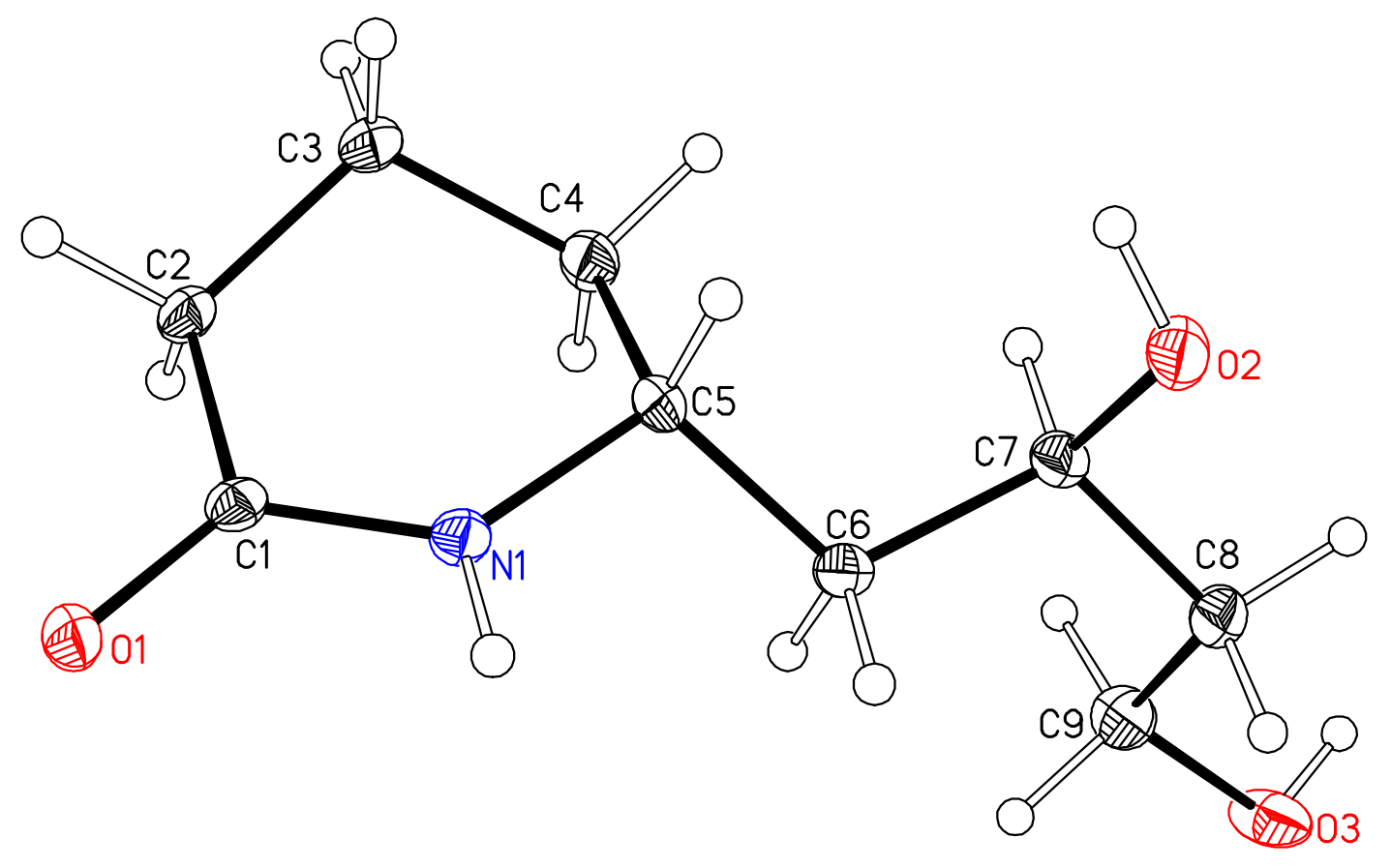


Table 1. Crystal data and structure refinement for x0353.

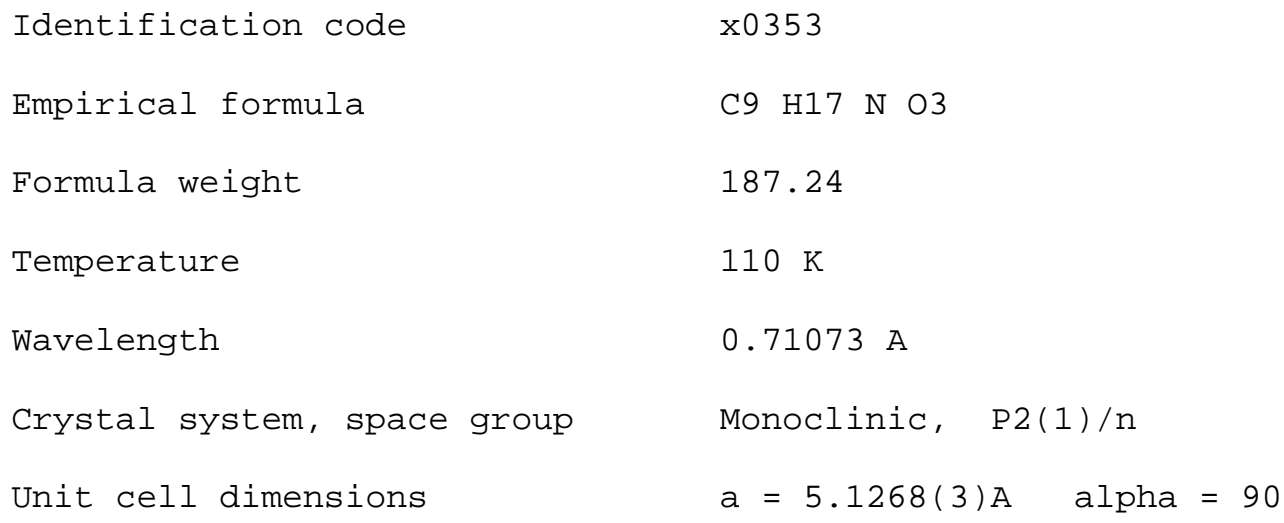


Table 2. Atomic coordinates ( $\mathrm{x}$ 10^4) and equivalent isotropic

displacement parameters $\left(A^{\wedge} 2 \times 10^{\wedge} 3\right)$ for $\times 0353$.

$\mathrm{U}(\mathrm{eq})$ is defined as one third of the trace of the orthogonalized

Uij tensor.

\begin{tabular}{|c|c|c|c|c|}
\hline & $\mathrm{x}$ & $\mathrm{Y}$ & $z$ & $\mathrm{U}(\mathrm{eq})$ \\
\hline$O(1)$ & $2004(3)$ & $315(1)$ & $8352(2)$ & $16(1)$ \\
\hline$O(2)$ & $4145(3)$ & $-2039(1)$ & $9938(2)$ & $20(1)$ \\
\hline$O(3)$ & $1817(3)$ & $-2094(1)$ & $15951(2)$ & $25(1)$ \\
\hline $\mathrm{N}(1)$ & $2501(3)$ & $-503(1)$ & $8953(2)$ & $12(1)$ \\
\hline$C(1)$ & $1026(3)$ & $-111(1)$ & $8060(2)$ & $12(1)$ \\
\hline$C(2)$ & $-1890(4)$ & $-193(1)$ & $6689(2)$ & $14(1)$ \\
\hline$C(3)$ & $-2506(4)$ & $-726(1)$ & $6014(3)$ & $17(1)$ \\
\hline$C(4)$ & $-1469(4)$ & $-1071(1)$ & $7799(3)$ & $15(1)$ \\
\hline$C(5)$ & $1641(4)$ & $-1023(1)$ & $8785(2)$ & $12(1)$ \\
\hline$C(6)$ & $2688(4)$ & $-1250(1)$ & $10868(2)$ & $13(1)$ \\
\hline$C(7)$ & $2367(4)$ & $-1811(1)$ & $10883(2)$ & $14(1)$ \\
\hline$C(8)$ & $3147(4)$ & $-2027(1)$ & $12967(3)$ & $17(1)$ \\
\hline$C(9)$ & $1418(4)$ & $-1826(1)$ & $14152(3)$ & $19(1)$ \\
\hline
\end{tabular}


Table 3. Bond lengths [A] and angles [deg] for x0353.

\begin{tabular}{|c|c|}
\hline$O(1)-C(1)$ & $1.244(2)$ \\
\hline $\mathrm{O}(2)-\mathrm{C}(7)$ & $1.439(2)$ \\
\hline $\mathrm{O}(2)-\mathrm{H}(2 \mathrm{O})$ & $0.82(3)$ \\
\hline$O(3)-C(9)$ & $1.428(2)$ \\
\hline $\mathrm{O}(3)-\mathrm{H}(3 \mathrm{O})$ & $0.84(3)$ \\
\hline $\mathrm{N}(1)-\mathrm{C}(1)$ & $1.336(2)$ \\
\hline$N(1)-C(5)$ & $1.467(2)$ \\
\hline $\mathrm{N}(1)-\mathrm{H}(1 \mathrm{~N})$ & $0.86(2)$ \\
\hline$C(1)-C(2)$ & $1.508(2)$ \\
\hline$C(2)-C(3)$ & $1.520(2)$ \\
\hline $\mathrm{C}(2)-\mathrm{H}(2 \mathrm{~A})$ & 0.9900 \\
\hline $\mathrm{C}(2)-\mathrm{H}(2 \mathrm{~B})$ & 0.9900 \\
\hline$C(3)-C(4)$ & $1.524(2)$ \\
\hline$C(3)-H(3 A)$ & 0.9900 \\
\hline$C(3)-H(3 B)$ & 0.9900 \\
\hline$C(4)-C(5)$ & $1.521(2)$ \\
\hline $\mathrm{C}(4)-\mathrm{H}(4 \mathrm{~A})$ & 0.9900 \\
\hline $\mathrm{C}(4)-\mathrm{H}(4 \mathrm{~B})$ & 0.9900 \\
\hline$C(5)-C(6)$ & $1.530(2)$ \\
\hline$C(5)-H(5 A)$ & 1.0000 \\
\hline$C(6)-C(7)$ & $1.526(2)$ \\
\hline$C(6)-H(6 A)$ & 0.9900 \\
\hline$C(6)-H(6 B)$ & 0.9900 \\
\hline$C(7)-C(8)$ & $1.522(2)$ \\
\hline$C(7)-H(7 A)$ & 1.0000 \\
\hline$C(8)-C(9)$ & $1.513(3)$ \\
\hline $\mathrm{C}(8)-\mathrm{H}(8 \mathrm{~A})$ & 0.9900 \\
\hline $\mathrm{C}(8)-\mathrm{H}(8 \mathrm{~B})$ & 0.9900 \\
\hline $\mathrm{C}(9)-\mathrm{H}(9 \mathrm{~A})$ & 0.9900 \\
\hline $\mathrm{C}(9)-\mathrm{H}(9 \mathrm{~B})$ & 0.9900 \\
\hline $\mathrm{C}(7)-\mathrm{O}(2)-\mathrm{H}(2 \mathrm{O})$ & $108.9(18)$ \\
\hline $\mathrm{C}(9)-\mathrm{O}(3)-\mathrm{H}(30)$ & $109.1(17)$ \\
\hline$C(1)-N(1)-C(5)$ & $127.93(15)$ \\
\hline $\mathrm{C}(1)-\mathrm{N}(1)-\mathrm{H}(1 \mathrm{~N})$ & $117.0(15)$ \\
\hline $\mathrm{C}(5)-\mathrm{N}(1)-\mathrm{H}(1 \mathrm{~N})$ & $115.1(15)$ \\
\hline $\mathrm{O}(1)-\mathrm{C}(1)-\mathrm{N}(1)$ & $121.36(16)$ \\
\hline$O(1)-C(1)-C(2)$ & $120.20(15)$ \\
\hline $\mathrm{N}(1)-\mathrm{C}(1)-\mathrm{C}(2)$ & $118.43(15)$ \\
\hline$C(1)-C(2)-C(3)$ & $113.74(14)$ \\
\hline $\mathrm{C}(1)-\mathrm{C}(2)-\mathrm{H}(2 \mathrm{~A})$ & 108.8 \\
\hline $\mathrm{C}(3)-\mathrm{C}(2)-\mathrm{H}(2 \mathrm{~A})$ & 108.8 \\
\hline $\mathrm{C}(1)-\mathrm{C}(2)-\mathrm{H}(2 \mathrm{~B})$ & 108.8 \\
\hline $\mathrm{C}(3)-\mathrm{C}(2)-\mathrm{H}(2 \mathrm{~B})$ & 108.8 \\
\hline $\mathrm{H}(2 \mathrm{~A})-\mathrm{C}(2)-\mathrm{H}(2 \mathrm{~B})$ & 107.7 \\
\hline$C(2)-C(3)-C(4)$ & $109.67(14)$ \\
\hline $\mathrm{C}(2)-\mathrm{C}(3)-\mathrm{H}(3 \mathrm{~A})$ & 109.7 \\
\hline $\mathrm{C}(4)-\mathrm{C}(3)-\mathrm{H}(3 \mathrm{~A})$ & 109.7 \\
\hline$C(2)-C(3)-H(3 B)$ & 109.7 \\
\hline $\mathrm{C}(4)-\mathrm{C}(3)-\mathrm{H}(3 \mathrm{~B})$ & 109.7 \\
\hline $\mathrm{H}(3 \mathrm{~A})-\mathrm{C}(3)-\mathrm{H}(3 \mathrm{~B})$ & 108.2 \\
\hline$C(5)-C(4)-C(3)$ & $110.93(15)$ \\
\hline
\end{tabular}




\begin{tabular}{|c|c|}
\hline$C(5)-C(4)-H(4 A)$ & 109.5 \\
\hline $\mathrm{C}(3)-\mathrm{C}(4)-\mathrm{H}(4 \mathrm{~A})$ & 109.5 \\
\hline $\mathrm{C}(5)-\mathrm{C}(4)-\mathrm{H}(4 \mathrm{~B})$ & 109.5 \\
\hline$C(3)-C(4)-H(4 B)$ & 109.5 \\
\hline $\mathrm{H}(4 \mathrm{~A})-\mathrm{C}(4)-\mathrm{H}(4 \mathrm{~B})$ & 108.0 \\
\hline$N(1)-C(5)-C(4)$ & $111.10(14)$ \\
\hline$N(1)-C(5)-C(6)$ & $107.73(13)$ \\
\hline$C(4)-C(5)-C(6)$ & $113.11(14)$ \\
\hline $\mathrm{N}(1)-\mathrm{C}(5)-\mathrm{H}(5 \mathrm{~A})$ & 108.3 \\
\hline$C(4)-C(5)-H(5 A)$ & 108.3 \\
\hline$C(6)-C(5)-H(5 A)$ & 108.3 \\
\hline$C(7)-C(6)-C(5)$ & $113.61(14)$ \\
\hline$C(7)-C(6)-H(6 A)$ & 108.8 \\
\hline$C(5)-C(6)-H(6 A)$ & 108.8 \\
\hline$C(7)-C(6)-H(6 B)$ & 108.8 \\
\hline$C(5)-C(6)-H(6 B)$ & 108.8 \\
\hline $\mathrm{H}(6 \mathrm{~A})-\mathrm{C}(6)-\mathrm{H}(6 \mathrm{~B})$ & 107.7 \\
\hline $\mathrm{O}(2)-\mathrm{C}(7)-\mathrm{C}(8)$ & $106.87(14)$ \\
\hline $\mathrm{O}(2)-\mathrm{C}(7)-\mathrm{C}(6)$ & $109.60(14)$ \\
\hline$C(8)-C(7)-C(6)$ & $113.25(14)$ \\
\hline $\mathrm{O}(2)-\mathrm{C}(7)-\mathrm{H}(7 \mathrm{~A})$ & 109.0 \\
\hline$C(8)-C(7)-H(7 A)$ & 109.0 \\
\hline$C(6)-C(7)-H(7 A)$ & 109.0 \\
\hline$C(9)-C(8)-C(7)$ & $113.18(15)$ \\
\hline $\mathrm{C}(9)-\mathrm{C}(8)-\mathrm{H}(8 \mathrm{~A})$ & 108.9 \\
\hline $\mathrm{C}(7)-\mathrm{C}(8)-\mathrm{H}(8 \mathrm{~A})$ & 108.9 \\
\hline$C(9)-C(8)-H(8 B)$ & 108.9 \\
\hline $\mathrm{C}(7)-\mathrm{C}(8)-\mathrm{H}(8 \mathrm{~B})$ & 108.9 \\
\hline $\mathrm{H}(8 \mathrm{~A})-\mathrm{C}(8)-\mathrm{H}(8 \mathrm{~B})$ & 107.8 \\
\hline $\mathrm{O}(3)-\mathrm{C}(9)-\mathrm{C}(8)$ & $112.54(15)$ \\
\hline $\mathrm{O}(3)-\mathrm{C}(9)-\mathrm{H}(9 \mathrm{~A})$ & 109.1 \\
\hline $\mathrm{C}(8)-\mathrm{C}(9)-\mathrm{H}(9 \mathrm{~A})$ & 109.1 \\
\hline $\mathrm{O}(3)-\mathrm{C}(9)-\mathrm{H}(9 \mathrm{~B})$ & 109.1 \\
\hline $\mathrm{C}(8)-\mathrm{C}(9)-\mathrm{H}(9 \mathrm{~B})$ & 109.1 \\
\hline $\mathrm{H}(9 \mathrm{~A})-\mathrm{C}(9)-\mathrm{H}(9 \mathrm{~B})$ & 107.8 \\
\hline
\end{tabular}


Table 4. Anisotropic displacement parameters ( $\left.A^{\wedge} 2 \times 10^{\wedge} 3\right)$ for x0353.

The anisotropic displacement factor exponent takes the form:

$-2 \mathrm{pi}^{\wedge} 2$ [ h^2 $\mathrm{a}^{\star \wedge} 2 \mathrm{U} 11+\ldots+2 \mathrm{~h} \mathrm{k} \mathrm{a}^{\star} \mathrm{b}$ * U12 ]

\begin{tabular}{|c|c|c|c|c|c|c|}
\hline \multicolumn{7}{|l|}{ U12 } \\
\hline$O(1)$ & $16(1)$ & $12(1)$ & $16(1)$ & $1(1)$ & $2(1)$ & - \\
\hline \multicolumn{7}{|l|}{$2(1)$} \\
\hline$O(2)$ & $30(1)$ & $19(1)$ & $14(1)$ & $2(1)$ & $10(1)$ & \\
\hline \multicolumn{7}{|l|}{$10(1)$} \\
\hline$O(3)$ & $44(1)$ & $20(1)$ & $13(1)$ & $-2(1)$ & $12(1)$ & - \\
\hline \multicolumn{7}{|l|}{$13(1)$} \\
\hline $\mathrm{N}(1)$ & $10(1)$ & $13(1)$ & $12(1)$ & $0(1)$ & $2(1)$ & - \\
\hline \multicolumn{7}{|l|}{$2(1)$} \\
\hline $\mathrm{C}(1)$ & $13(1)$ & $14(1)$ & $9(1)$ & $-1(1)$ & $5(1)$ & \\
\hline \multicolumn{7}{|l|}{$1(1)$} \\
\hline $\mathrm{C}(2)$ & $12(1)$ & $15(1)$ & $11(1)$ & $2(1)$ & $1(1)$ & \\
\hline \multicolumn{7}{|l|}{$1(1)$} \\
\hline $\mathrm{C}(3)$ & $16(1)$ & $17(1)$ & $13(1)$ & $0(1)$ & $-2(1)$ & - \\
\hline \multicolumn{7}{|l|}{$1(1)$} \\
\hline $\mathrm{C}(4)$ & $14(1)$ & $12(1)$ & $17(1)$ & $0(1)$ & $2(1)$ & - \\
\hline \multicolumn{7}{|l|}{$3(1)$} \\
\hline$C(5)$ & $14(1)$ & $9(1)$ & $14(1)$ & $-1(1)$ & $4(1)$ & - \\
\hline \multicolumn{7}{|l|}{$1(1)$} \\
\hline$C(6)$ & $15(1)$ & $13(1)$ & $12(1)$ & $-1(1)$ & $3(1)$ & - \\
\hline \multicolumn{7}{|l|}{$1(1)$} \\
\hline $\mathrm{C}(7)$ & $17(1)$ & $13(1)$ & $12(1)$ & $-1(1)$ & $5(1)$ & \\
\hline \multicolumn{7}{|l|}{$1(1)$} \\
\hline $\mathrm{C}(8)$ & $21(1)$ & $15(1)$ & $14(1)$ & $2(1)$ & $5(1)$ & \\
\hline \multicolumn{7}{|l|}{$2(1)$} \\
\hline $\mathrm{C}(9)$ & $27(1)$ & $17(1)$ & $15(1)$ & $2(1)$ & $9(1)$ & - \\
\hline $2(1)$ & & & & & & \\
\hline
\end{tabular}

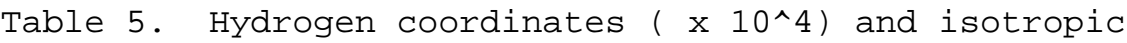
displacement parameters $\left(A^{\wedge} 2 \times 10^{\wedge} 3\right)$ for $\times 0353$.

\begin{tabular}{|c|c|c|c|c|}
\hline & $\mathrm{x}$ & $\mathrm{Y}$ & $\mathrm{z}$ & $\mathrm{U}(\mathrm{eq})$ \\
\hline $\mathrm{H}(2 \mathrm{O})$ & $3410(50)$ & $-2019(9)$ & $8730(40)$ & $36(7)$ \\
\hline $\mathrm{H}(30)$ & $1030(50)$ & $-2372(10)$ & $15680(40)$ & $40(7)$ \\
\hline
\end{tabular}




$\begin{array}{lcccc}H(1 \mathrm{~N}) & 4150(50) & -448(8) & 9730(30) & 22(6) \\ \mathrm{H}(2 \mathrm{~A}) & -2225 & 21 & 5504 & 16 \\ \mathrm{H}(2 \mathrm{~B}) & -3190 & -89 & 7377 & 16 \\ \mathrm{H}(3 \mathrm{~A}) & -1583 & -808 & 5033 & 20 \\ \mathrm{H}(3 \mathrm{~B}) & -4522 & -770 & 5366 & 20 \\ \mathrm{H}(4 \mathrm{~A}) & -2403 & -989 & 8773 & 18 \\ \mathrm{H}(4 \mathrm{~B}) & -1936 & -1417 & 7362 & 18 \\ \mathrm{H}(5 \mathrm{~A}) & 2563 & -1198 & 7938 & 16 \\ \mathrm{H}(6 \mathrm{~A}) & 4665 & -1165 & 11493 & 16 \\ \mathrm{H}(6 \mathrm{~B}) & 1664 & -1100 & 11684 & 17 \\ \mathrm{H}(7 \mathrm{~A}) & 406 & -1899 & 10130 & 20 \\ \mathrm{H}(8 \mathrm{~A}) & 2931 & -2391 & 12867 & 23 \\ \mathrm{H}(8 \mathrm{~B}) & 5118 & -1954 & 13688 & 23 \\ \mathrm{H}(9 \mathrm{~A}) & 1902 & -1474 & 14475 & \\ \mathrm{H}(9 \mathrm{~B}) & -558 & -1841 & 13327 & \\ & & & & \end{array}$


Table 6. Torsion angles [deg] for x0353.

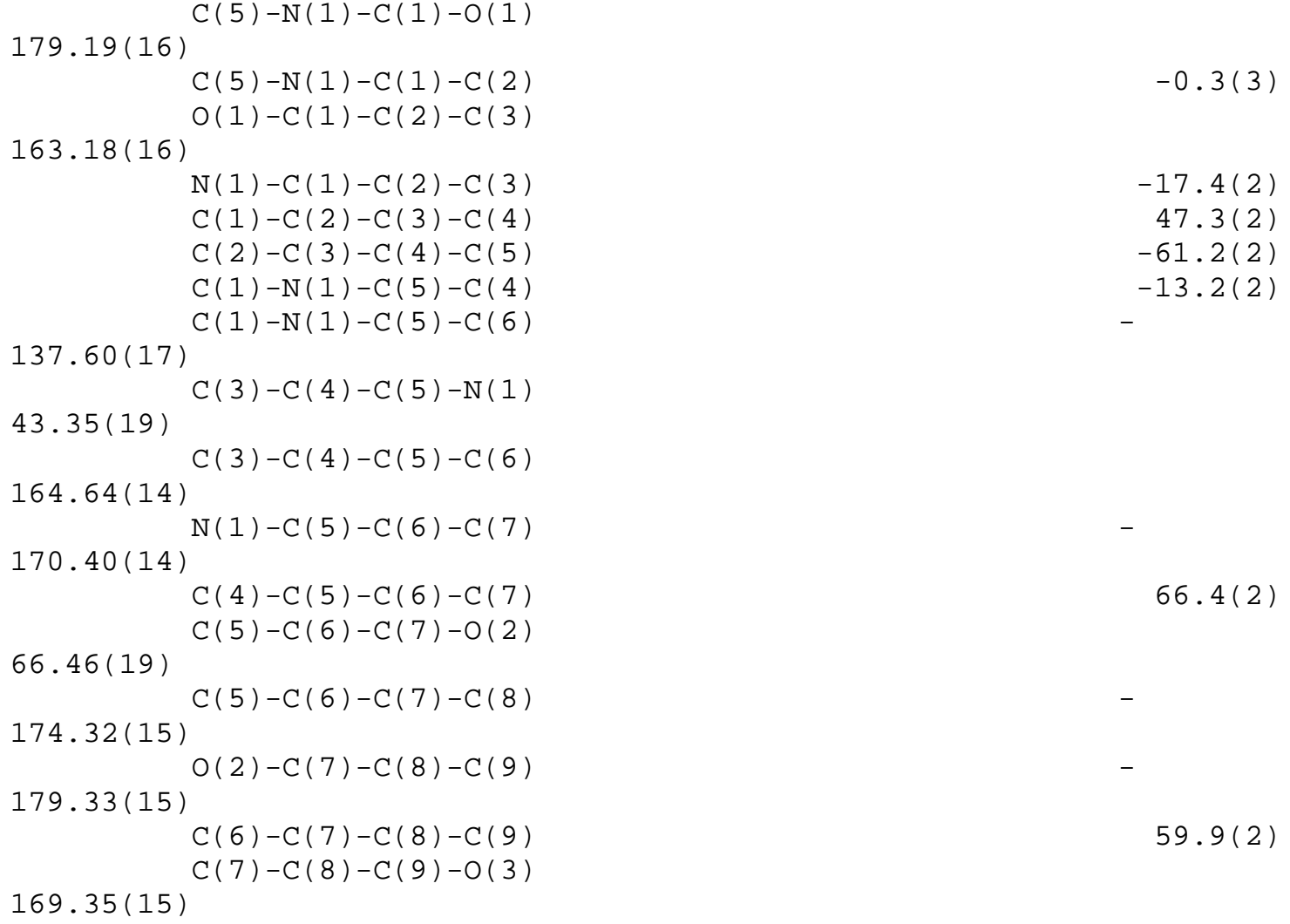

Table 7. Hydrogen bonds for x0353 [A and deg.].

\begin{tabular}{|c|c|c|c|}
\hline $\begin{aligned} & \mathrm{D}-\mathrm{H} \ldots \mathrm{A} \\
<(\mathrm{DHA}) & \end{aligned}$ & $d(D-H)$ & $d(H \ldots A)$ & $d(D \ldots A)$ \\
\hline $\begin{array}{l}\mathrm{O}(2)-\mathrm{H}(2 \mathrm{O}) \ldots \mathrm{O}(3) \# 1 \\
170(2)\end{array}$ & $0.82(3)$ & $1.89(3)$ & $2.6995(18)$ \\
\hline $\begin{array}{l}O(3)-H(30) \ldots O(2) \# 2 \\
176(3)\end{array}$ & $0.84(3)$ & $1.85(3)$ & $2.6916(19)$ \\
\hline $\begin{array}{l}\mathrm{N}(1)-\mathrm{H}(1 \mathrm{~N}) \ldots \mathrm{O}(1) \text { \#3 } \\
178(2)\end{array}$ & $0.86(2)$ & $2.03(2)$ & $2.8823(19)$ \\
\hline
\end{tabular}

Symmetry transformations used to generate equivalent atoms:

$\# 1 \mathrm{x}, \mathrm{y}, \mathrm{z}-1$ \#2 $\mathrm{x}-1 / 2,-\mathrm{y}-1 / 2, \mathrm{z}+1 / 2 \quad \# 3-\mathrm{x}+1,-\mathrm{y},-\mathrm{z}+2$ 


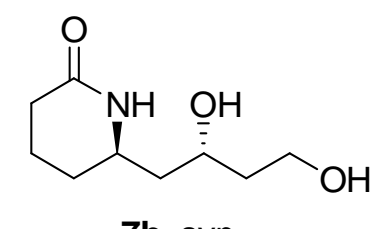

7b, syn

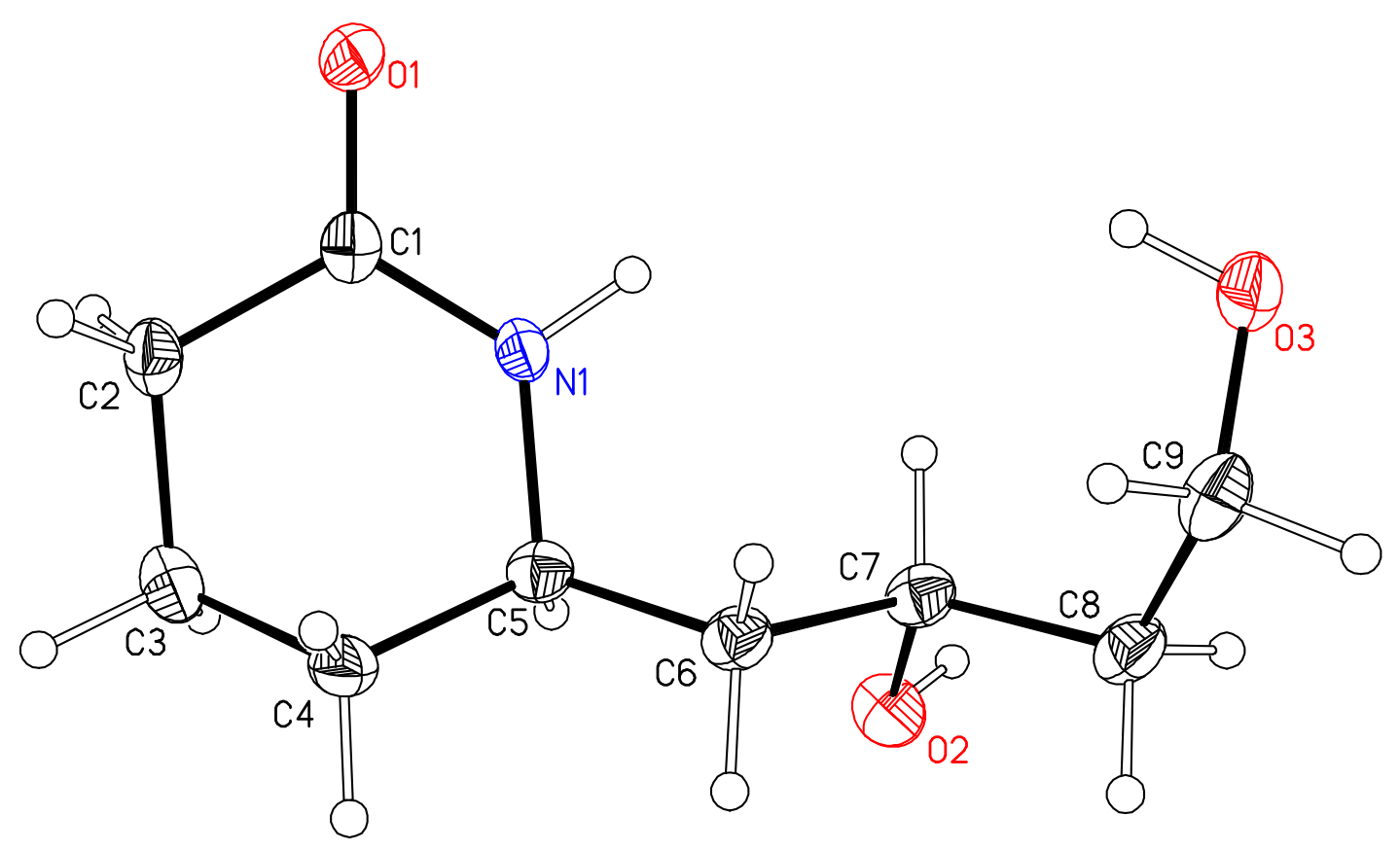


Table 1. Crystal data and structure refinement for x0354.

Identification code

Empirical formula

Formula weight

Temperature

Wavelength

Crystal system, space group

Unit cell dimensions

$65.30(3)$ deg.

88.44(2) deg.

$77.37(3) \mathrm{deg}$.

Volume

Z, Calculated density

Absorption coefficient

F $(000)$

Crystal size

Theta range for data collection

$11<=1<=12$

Limiting indices

Reflections collected / unique

Completeness to theta $=27.48$

Absorption correction

Max. and min. transmission

Refinement method

$\mathrm{F}^{\wedge} 2$

Data / restraints / parameters

Goodness-of-fit on $\mathrm{F}^{\wedge} 2$

Final $R$ indices [I>2sigma(I)]

R indices (all data)

Largest diff. peak and hole $x 0354$

C9 $\mathrm{H} 17 \mathrm{~N}$ O3

187.24

$110 \mathrm{~K}$

$0.71073 \mathrm{~A}$

Triclinic, $\quad \mathrm{P}-1$

$\mathrm{a}=6.1600(14)$ A alpha $=$

$\mathrm{b}=8.885(3) \mathrm{A} \quad$ beta $=$

$\mathrm{C}=9.942(3)$ A gamma $=$

$481.1(3) A^{\wedge} 3$

2, $1.292 \mathrm{Mg} / \mathrm{m}^{\wedge} 3$

$0.096 \mathrm{~mm}^{\wedge}-1$

204

$0.6400 \times 0.2898 \times 0.1346 \mathrm{~mm}$

3.94 to $27.48 \mathrm{deg}$.

$-7<=\mathrm{h}<=7, \quad-11<=\mathrm{k}<=11, \quad-$

$5205 / 2198$ [R(int) $=0.0313]$

$99.8 \%$

Analytical

0.991 and 0.974

Full-matrix least-squares on

2198 / 0 / 130

0.896

$\mathrm{R} 1=0.0393, \mathrm{wR} 2=0.0812$

$\mathrm{R} 1=0.0749, \mathrm{wR} 2=0.0899$

0.246 and -0.217 e. $A^{\wedge}-3$ 
Table 2. Atomic coordinates ( $\mathrm{x}$ 10^4) and equivalent isotropic

displacement parameters $\left(A^{\wedge} 2 \times 10^{\wedge} 3\right)$ for $\times 0354$.

$\mathrm{U}(\mathrm{eq})$ is defined as one third of the trace of the orthogonalized

Uij tensor.

\begin{tabular}{|c|c|c|c|c|}
\hline & $\mathrm{x}$ & Y & z & $\mathrm{U}$ (eq) \\
\hline$O(1)$ & $12302(2)$ & $-1582(1)$ & $6066(1)$ & $23(1)$ \\
\hline$O(2)$ & $5570(2)$ & $2948(1)$ & 7839 (1) & $26(1)$ \\
\hline$O(3)$ & $3710(2)$ & 3777 (1) & 3426 (1) & $24(1)$ \\
\hline $\mathrm{N}(1)$ & $9393(2)$ & $-460(2)$ & $7022(2)$ & $19(1)$ \\
\hline$C(1)$ & $11224(2)$ & $-1639(2)$ & $7168(2)$ & $18(1)$ \\
\hline $\mathrm{C}(2)$ & $11987(2)$ & $-3088(2)$ & $8664(2)$ & $22(1)$ \\
\hline$C(3)$ & $10590(2)$ & $-3004(2)$ & $9925(2)$ & $23(1)$ \\
\hline$C(4)$ & $8152(2)$ & $-2244(2)$ & $9374(2)$ & $21(1)$ \\
\hline$C(5)$ & $7915(2)$ & $-439(2)$ & $8209(2)$ & $18(1)$ \\
\hline$C(6)$ & $5540(2)$ & $455(2)$ & $7545(2)$ & $20(1)$ \\
\hline$C(7)$ & $5180(2)$ & $2380(2)$ & $6734(2)$ & $20(1)$ \\
\hline$C(8)$ & $2841(2)$ & $3249(2)$ & $5976(2)$ & $23(1)$ \\
\hline$C(9)$ & $2382(2)$ & $2998(2)$ & $4603(2)$ & $25(1)$ \\
\hline
\end{tabular}


Table 3. Bond lengths [A] and angles [deg] for x0354.

\begin{tabular}{|c|c|}
\hline$O(1)-C(1)$ & $1.2550(17)$ \\
\hline $\mathrm{O}(2)-\mathrm{C}(7)$ & $1.4316(18)$ \\
\hline $\mathrm{O}(2)-\mathrm{H}(2 \mathrm{O})$ & $0.83(2)$ \\
\hline$O(3)-C(9)$ & $1.4274(18)$ \\
\hline $\mathrm{O}(3)-\mathrm{H}(3 \mathrm{O})$ & $0.86(2)$ \\
\hline $\mathrm{N}(1)-\mathrm{C}(1)$ & $1.3248(18)$ \\
\hline$N(1)-C(5)$ & $1.4747(18)$ \\
\hline $\mathrm{N}(1)-\mathrm{H}(1 \mathrm{~N})$ & $0.895(15)$ \\
\hline$C(1)-C(2)$ & $1.504(2)$ \\
\hline$C(2)-C(3)$ & $1.519(2)$ \\
\hline $\mathrm{C}(2)-\mathrm{H}(2 \mathrm{~A})$ & 0.9900 \\
\hline $\mathrm{C}(2)-\mathrm{H}(2 \mathrm{~B})$ & 0.9900 \\
\hline$C(3)-C(4)$ & $1.517(2)$ \\
\hline$C(3)-H(3 A)$ & 0.9900 \\
\hline$C(3)-H(3 B)$ & 0.9900 \\
\hline$C(4)-C(5)$ & $1.513(2)$ \\
\hline $\mathrm{C}(4)-\mathrm{H}(4 \mathrm{~A})$ & 0.9900 \\
\hline $\mathrm{C}(4)-\mathrm{H}(4 \mathrm{~B})$ & 0.9900 \\
\hline$C(5)-C(6)$ & $1.521(2)$ \\
\hline$C(5)-H(5 A)$ & 1.0000 \\
\hline$C(6)-C(7)$ & $1.522(2)$ \\
\hline$C(6)-H(6 A)$ & 0.9900 \\
\hline$C(6)-H(6 B)$ & 0.9900 \\
\hline$C(7)-C(8)$ & $1.526(2)$ \\
\hline$C(7)-H(7 A)$ & 1.0000 \\
\hline$C(8)-C(9)$ & $1.515(2)$ \\
\hline $\mathrm{C}(8)-\mathrm{H}(8 \mathrm{~A})$ & 0.9900 \\
\hline $\mathrm{C}(8)-\mathrm{H}(8 \mathrm{~B})$ & 0.9900 \\
\hline $\mathrm{C}(9)-\mathrm{H}(9 \mathrm{~A})$ & 0.9900 \\
\hline$C(9)-H(9 B)$ & 0.9900 \\
\hline $\mathrm{C}(7)-\mathrm{O}(2)-\mathrm{H}(2 \mathrm{O})$ & $107.0(13)$ \\
\hline $\mathrm{C}(9)-\mathrm{O}(3)-\mathrm{H}(3 \mathrm{O})$ & $107.1(13)$ \\
\hline $\mathrm{C}(1)-\mathrm{N}(1)-\mathrm{C}(5)$ & $125.86(13)$ \\
\hline $\mathrm{C}(1)-\mathrm{N}(1)-\mathrm{H}(1 \mathrm{~N})$ & $114.5(9)$ \\
\hline $\mathrm{C}(5)-\mathrm{N}(1)-\mathrm{H}(1 \mathrm{~N})$ & $119.0(9)$ \\
\hline $\mathrm{O}(1)-\mathrm{C}(1)-\mathrm{N}(1)$ & $120.72(14)$ \\
\hline$O(1)-C(1)-C(2)$ & $119.56(13)$ \\
\hline $\mathrm{N}(1)-\mathrm{C}(1)-\mathrm{C}(2)$ & $119.70(14)$ \\
\hline$C(1)-C(2)-C(3)$ & $115.03(13)$ \\
\hline $\mathrm{C}(1)-\mathrm{C}(2)-\mathrm{H}(2 \mathrm{~A})$ & 108.5 \\
\hline $\mathrm{C}(3)-\mathrm{C}(2)-\mathrm{H}(2 \mathrm{~A})$ & 108.5 \\
\hline $\mathrm{C}(1)-\mathrm{C}(2)-\mathrm{H}(2 \mathrm{~B})$ & 108.5 \\
\hline $\mathrm{C}(3)-\mathrm{C}(2)-\mathrm{H}(2 \mathrm{~B})$ & 108.5 \\
\hline $\mathrm{H}(2 \mathrm{~A})-\mathrm{C}(2)-\mathrm{H}(2 \mathrm{~B})$ & 107.5 \\
\hline$C(4)-C(3)-C(2)$ & $110.37(13)$ \\
\hline $\mathrm{C}(4)-\mathrm{C}(3)-\mathrm{H}(3 \mathrm{~A})$ & 109.6 \\
\hline $\mathrm{C}(2)-\mathrm{C}(3)-\mathrm{H}(3 \mathrm{~A})$ & 109.6 \\
\hline$C(4)-C(3)-H(3 B)$ & 109.6 \\
\hline $\mathrm{C}(2)-\mathrm{C}(3)-\mathrm{H}(3 \mathrm{~B})$ & 109.6 \\
\hline $\mathrm{H}(3 \mathrm{~A})-\mathrm{C}(3)-\mathrm{H}(3 \mathrm{~B})$ & 108.1 \\
\hline$C(5)-C(4)-C(3)$ & $108.92(12)$ \\
\hline
\end{tabular}




\begin{tabular}{|c|c|}
\hline$C(5)-C(4)-H(4 A)$ & 109.9 \\
\hline $\mathrm{C}(3)-\mathrm{C}(4)-\mathrm{H}(4 \mathrm{~A})$ & 109.9 \\
\hline $\mathrm{C}(5)-\mathrm{C}(4)-\mathrm{H}(4 \mathrm{~B})$ & 109.9 \\
\hline$C(3)-C(4)-H(4 B)$ & 109.9 \\
\hline $\mathrm{H}(4 \mathrm{~A})-\mathrm{C}(4)-\mathrm{H}(4 \mathrm{~B})$ & 108.3 \\
\hline $\mathrm{N}(1)-\mathrm{C}(5)-\mathrm{C}(4)$ & $108.78(12)$ \\
\hline$N(1)-C(5)-C(6)$ & $109.77(12)$ \\
\hline$C(4)-C(5)-C(6)$ & $114.01(12)$ \\
\hline $\mathrm{N}(1)-\mathrm{C}(5)-\mathrm{H}(5 \mathrm{~A})$ & 108.0 \\
\hline $\mathrm{C}(4)-\mathrm{C}(5)-\mathrm{H}(5 \mathrm{~A})$ & 108.0 \\
\hline $\mathrm{C}(6)-\mathrm{C}(5)-\mathrm{H}(5 \mathrm{~A})$ & 108.0 \\
\hline$C(5)-C(6)-C(7)$ & $113.59(12)$ \\
\hline$C(5)-C(6)-H(6 A)$ & 108.8 \\
\hline $\mathrm{C}(7)-\mathrm{C}(6)-\mathrm{H}(6 \mathrm{~A})$ & 108.8 \\
\hline $\mathrm{C}(5)-\mathrm{C}(6)-\mathrm{H}(6 \mathrm{~B})$ & 108.8 \\
\hline$C(7)-C(6)-H(6 B)$ & 108.8 \\
\hline $\mathrm{H}(6 \mathrm{~A})-\mathrm{C}(6)-\mathrm{H}(6 \mathrm{~B})$ & 107.7 \\
\hline $\mathrm{O}(2)-\mathrm{C}(7)-\mathrm{C}(6)$ & $105.81(12)$ \\
\hline $\mathrm{O}(2)-\mathrm{C}(7)-\mathrm{C}(8)$ & $110.24(11)$ \\
\hline$C(6)-C(7)-C(8)$ & $113.03(12)$ \\
\hline $\mathrm{O}(2)-\mathrm{C}(7)-\mathrm{H}(7 \mathrm{~A})$ & 109.2 \\
\hline $\mathrm{C}(6)-\mathrm{C}(7)-\mathrm{H}(7 \mathrm{~A})$ & 109.2 \\
\hline $\mathrm{C}(8)-\mathrm{C}(7)-\mathrm{H}(7 \mathrm{~A})$ & 109.2 \\
\hline$C(9)-C(8)-C(7)$ & $114.98(12)$ \\
\hline $\mathrm{C}(9)-\mathrm{C}(8)-\mathrm{H}(8 \mathrm{~A})$ & 108.5 \\
\hline $\mathrm{C}(7)-\mathrm{C}(8)-\mathrm{H}(8 \mathrm{~A})$ & 108.5 \\
\hline $\mathrm{C}(9)-\mathrm{C}(8)-\mathrm{H}(8 \mathrm{~B})$ & 108.5 \\
\hline $\mathrm{C}(7)-\mathrm{C}(8)-\mathrm{H}(8 \mathrm{~B})$ & 108.5 \\
\hline $\mathrm{H}(8 \mathrm{~A})-\mathrm{C}(8)-\mathrm{H}(8 \mathrm{~B})$ & 107.5 \\
\hline $\mathrm{O}(3)-\mathrm{C}(9)-\mathrm{C}(8)$ & $112.37(12)$ \\
\hline $\mathrm{O}(3)-\mathrm{C}(9)-\mathrm{H}(9 \mathrm{~A})$ & 109.1 \\
\hline $\mathrm{C}(8)-\mathrm{C}(9)-\mathrm{H}(9 \mathrm{~A})$ & 109.1 \\
\hline $\mathrm{O}(3)-\mathrm{C}(9)-\mathrm{H}(9 \mathrm{~B})$ & 109.1 \\
\hline $\mathrm{C}(8)-\mathrm{C}(9)-\mathrm{H}(9 \mathrm{~B})$ & 109.1 \\
\hline $\mathrm{H}(9 \mathrm{~A})-\mathrm{C}(9)-\mathrm{H}(9 \mathrm{~B})$ & 107.9 \\
\hline
\end{tabular}


Table 4. Anisotropic displacement parameters ( $\left.A^{\wedge} 2 \times 10^{\wedge} 3\right)$ for x0354.

The anisotropic displacement factor exponent takes the form:

$-2 \mathrm{pi}^{\wedge} 2\left[\mathrm{~h}^{\wedge} 2 \mathrm{a}^{\star \wedge} 2 \mathrm{U} 11+\ldots+2 \mathrm{~h} \mathrm{k} \mathrm{a}^{\star} \mathrm{b} \mathrm{b}^{\star} \mathrm{U} 12\right]$

\begin{tabular}{|c|c|c|c|c|c|c|}
\hline U12 & U11 & $\mathrm{U} 22$ & U33 & $\mathrm{U} 23$ & U13 & \\
\hline$O(1)$ & $16(1)$ & $23(1)$ & $23(1)$ & $-7(1)$ & $4(1)$ & - \\
\hline $2(1)$ & & & & & & \\
\hline $7(1)^{O(2)}$ & $37(1)$ & $19(1)$ & $24(1)$ & $-12(1)$ & $3(1)$ & - \\
\hline $1(1)^{O(3)}$ & $23(1)$ & $20(1)$ & $24(1)$ & $-8(1)$ & $-1(1)$ & \\
\hline $2(1)^{\mathrm{N}(1)}$ & $17(1)$ & $16(1)$ & $16(1)$ & $-2(1)$ & $1(1)$ & - \\
\hline $6(1)^{C(1)}$ & $14(1)$ & $16(1)$ & $23(1)$ & $-7(1)$ & $-1(1)$ & - \\
\hline $3(1)^{C(2)}$ & $17(1)$ & $18(1)$ & $26(1)$ & $-4(1)$ & $-2(1)$ & - \\
\hline $5(1)^{C(3)}$ & $30(1)$ & $18(1)$ & $20(1)$ & $-6(1)$ & $-3(1)$ & - \\
\hline $5(1)^{C(4)}$ & $26(1)$ & $19(1)$ & $18(1)$ & $-8(1)$ & $5(1)$ & - \\
\hline $5(1)^{C(5)}$ & $20(1)$ & $19(1)$ & $19(1)$ & $-11(1)$ & $2(1)$ & - \\
\hline $6(1)^{C(6)}$ & $20(1)$ & $19(1)$ & $23(1)$ & $-10(1)$ & $5(1)$ & - \\
\hline $5(1)^{C(7)}$ & $21(1)$ & $19(1)$ & $23(1)$ & $-12(1)$ & $4(1)$ & - \\
\hline $2(1)^{C(8)}$ & $21(1)$ & $20(1)$ & $29(1)$ & $-12(1)$ & $5(1)$ & - \\
\hline $3(1)^{C(9)}$ & $17(1)$ & $24(1)$ & $34(1)$ & $-13(1)$ & $-1(1)$ & - \\
\hline
\end{tabular}

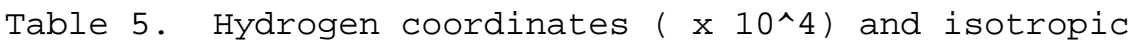
displacement parameters $\left(A^{\wedge} 2 \times 10^{\wedge} 3\right)$ for $\times 0354$.

\begin{tabular}{ccccc}
\hline & $x$ & $y$ & \\
& & & \\
& & & \\
& & & \\
& & & & \\
$\mathrm{H}(20)$ & $5850(30)$ & $3910(30)$ & $7400(20)$ & $49(6)$ \\
$\mathrm{H}(30)$ & $4990(30)$ & $3080(30)$ & $3590(20)$ & $62(7)$
\end{tabular}




$\begin{array}{lcrrr}\mathrm{H}(1 \mathrm{~N}) & 8950(20) & 280(18) & 6084(17) & 13(4) \\ \mathrm{H}(2 \mathrm{~A}) & 13546 & -3119 & 8913 & 26 \\ \mathrm{H}(2 \mathrm{~B}) & 11982 & -4167 & 8595 & 26 \\ \mathrm{H}(3 \mathrm{~A}) & 10752 & -4163 & 10728 & 28 \\ \mathrm{H}(3 \mathrm{~B}) & 11126 & -2299 & 10336 & 28 \\ \mathrm{H}(4 \mathrm{~A}) & 7616 & -2931 & 8943 & 25 \\ \mathrm{H}(4 \mathrm{~B}) & 7243 & -2248 & 10211 & 25 \\ \mathrm{H}(5 \mathrm{~A}) & 8431 & 228 & 8684 & 22 \\ \mathrm{H}(6 \mathrm{~A}) & 4509 & 178 & 8349 & 24 \\ \mathrm{H}(6 \mathrm{~B}) & 5159 & 10 & 6842 & 24 \\ \mathrm{H}(7 \mathrm{~A}) & 6302 & 2667 & 5975 & 27 \\ \mathrm{H}(8 \mathrm{~A}) & 1744 & 2813 & 6701 & 27 \\ \mathrm{H}(8 \mathrm{~B}) & 2597 & 4484 & 5697 & 30 \\ \mathrm{H}(9 \mathrm{~A}) & 2686 & 1765 & 4861 & 30 \\ \mathrm{H}(9 \mathrm{~B}) & 785 & 3488 & 4259 & \end{array}$


Table 6. Torsion angles [deg] for x0354.

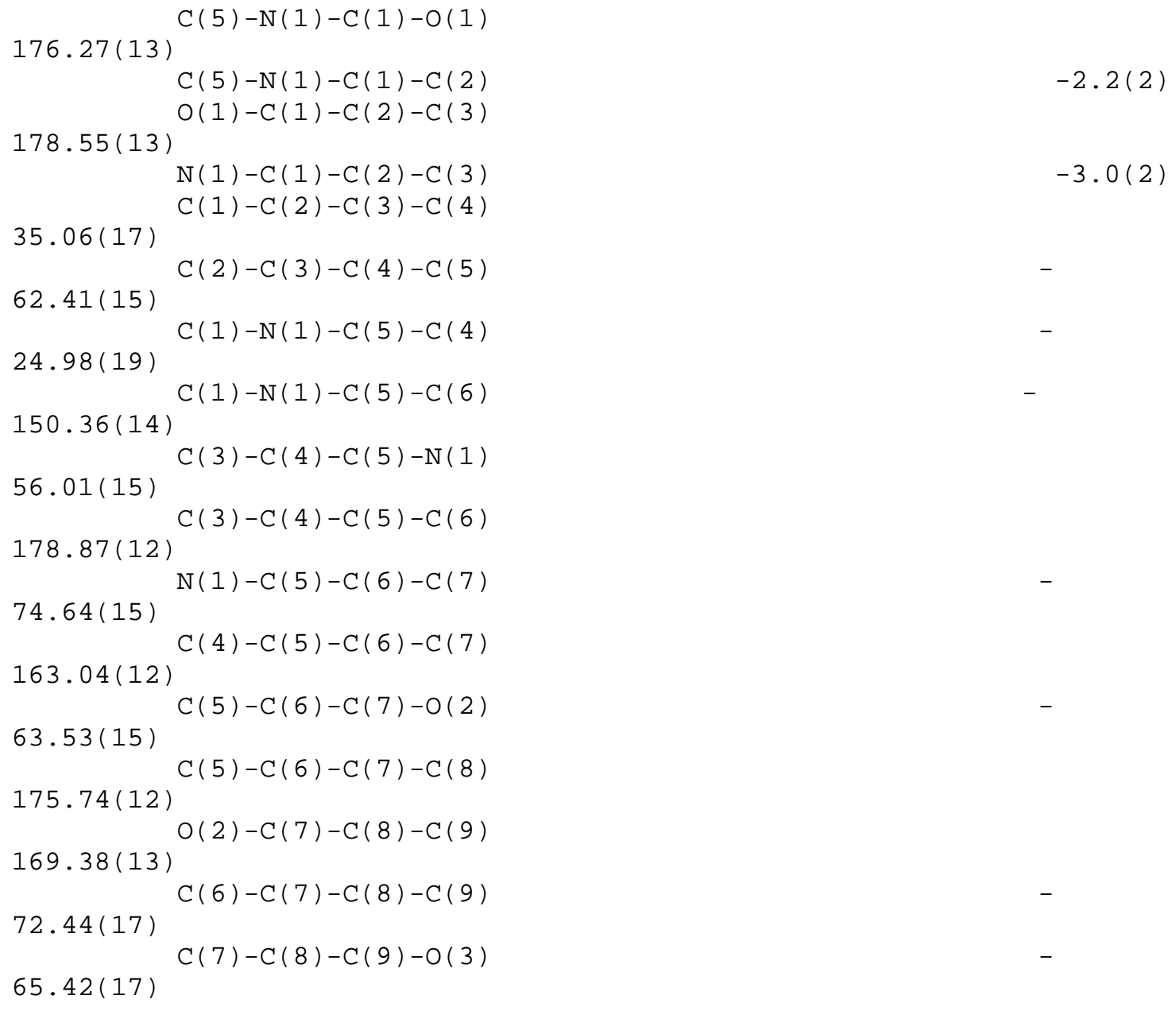

Table 7. Hydrogen bonds for x0354 [A and deg.].

\begin{tabular}{llll}
\hline & & & \\
& \\
$\begin{array}{c}\mathrm{D}-\mathrm{H} \ldots \mathrm{A} \\
<(\mathrm{DHA})\end{array}$ & $\mathrm{d}(\mathrm{D}-\mathrm{H})$ & $\mathrm{d}(\mathrm{H} \ldots \mathrm{A})$ & $\mathrm{d}(\mathrm{D} \ldots \mathrm{A})$ \\
$\mathrm{O}(2)-\mathrm{H}(2 \mathrm{O}) \ldots \mathrm{O}(3) \# 1$ & $0.83(2)$ & $1.95(2)$ & $2.7785(19)$ \\
$\begin{array}{l}171.9(18) \\
\quad \mathrm{O}(3)-\mathrm{H}(30) \ldots \mathrm{O}(1) \# 2\end{array}$ & $0.86(2)$ & $1.83(2)$ & $2.6906(17)$ \\
$\begin{array}{l}178.9(19) \\
\mathrm{N}(1)-\mathrm{H}(1 \mathrm{~N}) \ldots \mathrm{O}(1) \# 2\end{array}$ & $0.895(15)$ & $2.033(16)$ & $2.915(2)$ \\
$168.6(12)$ & & &
\end{tabular}


Symmetry transformations used to generate equivalent atoms: \#1 $-x+1,-y+1,-z+1 \quad \# 2-x+2,-y,-z+1$ 